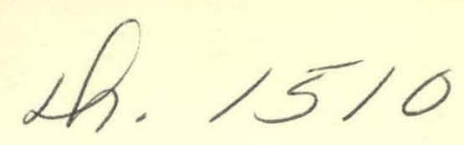

ORNL/TM-5873

\title{
In-Phantom Spectrometry of Medical Diagnostic X Rays
}

Paul Stewart Stansbury

\section{OAK RIDGE NATIONAL LABORATORY}




\section{DISCLAIMER}

This report was prepared as an account of work sponsored by an agency of the United States Government. Neither the United States Government nor any agency Thereof, nor any of their employees, makes any warranty, express or implied, or assumes any legal liability or responsibility for the accuracy, completeness, or usefulness of any information, apparatus, product, or process disclosed, or represents that its use would not infringe privately owned rights. Reference herein to any specific commercial product, process, or service by trade name, trademark, manufacturer, or otherwise does not necessarily constitute or imply its endorsement, recommendation, or favoring by the United States Government or any agency thereof. The views and opinions of authors expressed herein do not necessarily state or reflect those of the United States Government or any agency thereof. 


\section{DISCLAIMER}

Portions of this document may be illegible in electronic image products. Images are produced from the best available original document. 


\title{
Printed in the United States of America. Available from National Technical Information Service \\ U.S. Department of Commerce 5285 Port Royal Road, Springfield, Virginia 22161 \\ Price: Printed Copy $\$ 8.00$; Microfiche $\$ 3.00$
}

\begin{abstract}
This report was prepared as an accuunl ul wurk sponsored by the United States Government. Neither the United States nor the Energy Research and Development Administration/United States Nuclear Regulatory Commission, nor any of their employees, nor any of thcir contractors, subcuntraclurs, or thelr employees, makes any warranty. express or impliẹ,, or assumes any legal liability or responsibility for the accuracy, completeness or usefulness of any information. apparatıs, prndiıt or process disclosed, or represents that its use would not infringe privately owned rights.
\end{abstract}


ORNL/TM-5873

Dist. Category UC -41

Contract No. W-7405-eng-26

HEALTH AND SAFETY RESEARCH DIVISION

\section{IN-PHANTOM SPECTROMETRY OF MEDICAL DIAGNOSTIC $X$ RAYS}

\section{Paul Stewart Stansbury}

Submitted by P. S. Stansbury to

The Faculty of the Division of Graduate Studies and Research

in Partial Fulfillment of the Requirements for the Degree of Doctor of Philosophy in the School of Nuclear Engineering Georgia Institute of Technology

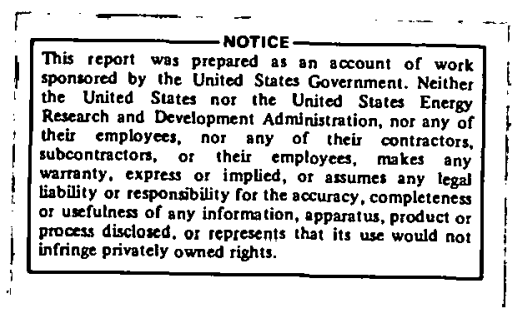

Date Published: October 1977

NOTICE This document contains information of a preliminary nature. It is subject to revision or correction and therefore does not represent a final renort

OAK RIDGE NATIONAL LABORATORY

Oak Ridge, Tennessee 37830 operated by UNION CARBIDE CORPORATION for the

ENERGY RESEARCH AND DEVELOPMENT ADMINISTRATION 
THIS PAGE

\section{WAS INTENTIONALLY \\ LEFT BLANK}




\section{ACKNOWLEDGMENTS}

The author expresses his gratitude to F.W. Chambers, who first introduced him to health physics and medical physics. Deepest gratitude is expressed to J. W. Poston, who, as laboratory research advisor, provided the inspiration, support, understanding and criticism needed to bring this project to fruition. Thanks are expressed to K. Z. Morgan, thesis advisor, and G. G. Eichholz, thesis committee member, for their interest and sagacious advice. The author thanks J. A. Auxier for his help in securing the author's appointment as an Oak Ridge Associated Universities Graduate Laboratory Participant and for supporting this project as part of the research of the Health Physics Division of the Oak Ridge National Laboratory.

Thanks are expressed to J.H. Thorngate, D. J. Christian, and G. K. Schulze for their work on the design and development of the $x$-ray machine. H. S. Roach, B. G. Foutz and R. S. Jones provided valuable assistance in the construction of the phantom. The author especially thanks W. F. Bunch for his help with all of the "gadgetry" necessary for a project of this sort.

T. D. Jones, P. T. Perdue, and V. E. Anderson are to be thanked for their advice about dosimetry, mathematics and computer programming. The assistance of G. G. Warner and S. K. Penny in performing some of the computer-coded calculations needed in this study is gratefully acknowledged. Thanks are expressed to C. H. Abner and S. R. Larkins for their engineering efforts, to C. C. Hall for his engineering of the safety interlocks, and to H. M. Butler and W. F. Ohnesorge for their advice 
and assistance with the health physics matters related to the project.

C. E. Bemis provided the advice, assistance, and equipment necessary to perform the high resolution spectrometry. In a similar manner, H. W. Dunn provided the use of an $x$-ray monochromator. Their essential help is acknowledged. The author thanks the other students who assisted him with various aspects of the project. They include S. M. Garry, H. N. Mei, W. L. Chen, D. P. Humer, and S. S. Jaeger. The author expresses his appreciation to the people who, in various capacities, helped "get things done." These people include J. P. Hickey, E. T. Loy, J. L. Malone, T. Neuman, F. Rau, C. E. McFarl and, G. E. Long, B. E. Thomas, C. C. Hayes, and others. Appreciation is expressed to the Georgia Institute of Terhnology for granting spccial permission to use pagination which conforms to ORNL standards.

Thanks are expressed to K. M. Branam and N. L. Barringer for their assistance in preparing drafts and the other paperwork generated in Lhe course of this project. Appreciation is expressed to B. S. Varnadorc for her help in preparing this manuscript. Finally, lile author thaniks his wife and daughter for their loving patience, understanding, and endurance.

This research was carried out at the Oak Ridge National Laboratory which is operated by the Energy Research and Development Administration under contrart with Union Carbide Corporation. 
TABLE OF CONTENTS

PAGE

ACKNOWLEDGEMENTS ........................

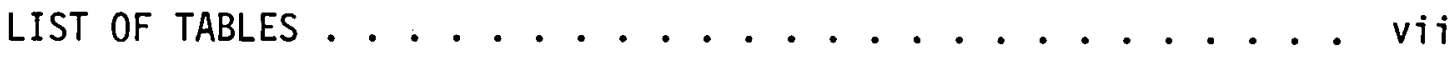

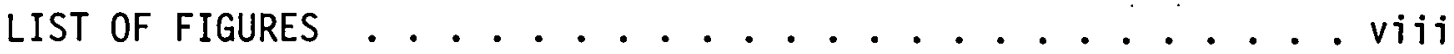

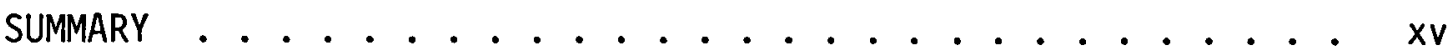

Chapter

I. INTRODUCTION ......................... I

II. THEORY ............................. 11

Production of $X$ Rays

Interaction of $X$ Rays with Matter

Monte Carlo Calculations

Phantom Measurements

Sodium Iodide Scintillation Spectrometry

III. APPARATUS AND PROCEDURES . . . . . . . . . . 35

$X$-Ray Facility

Spectrometer

Unfolding Procedure

MR. ADAM Phantom

IV. RESULTS AND CONCLUSIONS .............. 79

Outline of Spectral Measurements

Highlights of Results

Protective Measures

Effect of Depth of Penetration, Scattering Distance and Beam Size

Spectra Inside the Spine

Error Analys is

Comparison with BRH Calculations

V. FINAL SUMMARY .................. 129 Appendices

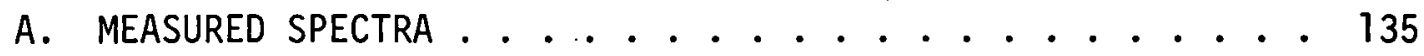

B. EFFECT OF FIELD SIZE .................. 192

BIBLIOGRAPHY .......................... 211

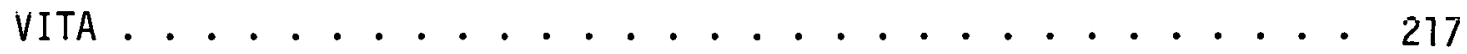




\section{THIS PAGE}

\section{WAS INTENTIONALLY \\ LEFT BLANK}


1. Coefficients and Parameters of Exponential Attenuation Equations of the Form $A=A_{0} \mathrm{e}^{-B X} \ldots \ldots 18$

2. Description of the Snyder-Fisher Phantom Subregions and their MR. ADAM Phantom Equivalents ........ 75

3. Calculated Partial Mass Attenuation Coefficients for MR. ADAM Phantom Materials and Comparison with the Coefficients for Snyder-Fisher Phantom -- Soft Tissue . . . . . . . 76

4. Calculated Partial Mass Attenuation Coefficients for MR. ADAM Phantom Materials and Comparison with the Coefficients for Snyder-Fisher Phantom -- Skeleton . . . . . . . . 77

5. Calculated Partial Mass Attenuation Coefficients for MR. ADAM Phantom Materials and Comparison with the Coefficients for Snyder-Fisher Phantom -- Lung . . . . . . . . 78

6. Summary of Probe Locations . . . . . . . . . . . . 82

7. Summary of Spectral Data for Testes and Right Ovary . . 94

8. Summary of Spectral Data for Heart Region, Fifth Lumbar Vertebra, and Thoracic Spine ........... 95

9. Summary of Spectral Data for Thyroid Gland and Eye Region. 96

10. Comparison of Ionization Chemaber Measurements with the Absorbed Dose Rate Determined from the Measured Spectrum at the Testes ................ 124

11. Comparison of Measured Absorbed Dose with BRH Calculations 126 


\section{LIST OF FIGURES}

FIGURE

PAGE

1. A Summary of BEIR Committee Estimates of Annual WholeBody Average Dose Rate in the United States in 1970 (BEIR72)................... 3

2. Mass Energy Absorption and Partiai Mass Attenuation Coefficients for Snyder-Fisher Soft Tissue (St74). . . . . 21

3. Floor Plan for the X-Ray Façility . . . . . . . . . 37

4. X-Ray Machine Schematic . . . . . . . . . . 38

5. Exposure Rate at a Target to Detector Distance of $100 \mathrm{~cm}$ as a Function of Generating Potential . . . . . . . . 41

6. Normalized Exposure Rate as a Function of Tube Current (Error Bars Correspond to $\pm 2 \%$ Plus the Least Significant Digit Uncertainty) . . . . . . . . . . . . . 42

7. X-Ray Machine Output Spectrum Measured with a Ge(L.i) Detector -- $60 \mathrm{kVcp}$.............. . 44

8. X-Ray Machine Output Spectrum Measured with a $\mathrm{Ge}(\mathrm{Li})$ Detector -- $80 \mathrm{kVcp}$.............. . 45

9. X-Ray Machine Output Spectrum Measured with a $\mathrm{Ge}(\mathrm{L} i)$ Detector -- $100 \mathrm{kVcp}$................ 46

10. Comparison of X-Ray Machine Output Spectrum with a Representative Diagnostic X-Ray Spectrum . . . . . . 49

11. $\mathrm{NaI}(\mathrm{T} \ell)$ Scintillation Detector .......... 51

12. Change in Mean of Pulse Height Distribution as a Function of Declination and Energy of Incident Beam (Data are Normalized so that Side Response Equals 100\%.). . . . . 54

13. Mean of Pulse Helght Distribution versus Energy for the Spherical $\mathrm{NaI}(\mathrm{T} \ell)$ Detector ............. . 57

14. Sigma of Pulse Height Distribution versus Energy for the Spherical $\mathrm{NaI}(\mathrm{Tl})$ Detector ............. 58 
FIGURE

PAGE

15. Calculated Distribution of the Energy Deposited in the NaI Crystal of the Detector by a Uniform Parallel Beam of $110 \mathrm{keV}$ Photons....................

16. Calculated Response Function for $110 \mathrm{keV}$ Uniformly Incident Photons ................

17. Comparison of Unfolded $\mathrm{NaI}(\mathrm{Tl})$ Spectrum with Spectrum Measured with a $\mathrm{Ge}(\mathrm{Li})$ Detector -- $60 \mathrm{kVcp} . . . . . .$.

18. Comparison of Unfolded $\mathrm{NaI}(\mathrm{Tl})$ Spectrum with Spectrum Measured with a $\mathrm{Ge}(\mathrm{Li})$ Detector -- $80 \mathrm{kVcp}$. . . . . . 68

19. An Example of Truncation and Extrapolation . . . . . 70

20. The MR. ADAM Phantom . . . . . . . . . . . 73

21. Data Acquisition and Processing Schematic . . . . . 80

22. Beam Location for Lower Spine $10 \times 10 \mathrm{~cm}$ Field (Center at $x=0, z=22)$............ 83

23. Beam Location for Full Spine $10 \times 44 \mathrm{~cm}$ Field (Center at $x=0, z=22)$..............

24. Beam Location for Abdominal $36 \times 44 \mathrm{~cm}$ Field (Center at $x=0, z=22$ ).................

25. Beam Location for Shoulder $36 \times 44 \mathrm{~cm}$ Field (Center at $x=14.3, z=61.5)$..............

26. Beam Location for Upper Gastro-Intestinal (Upper G.I.) $36 \times 22 \mathrm{~cm}$ Field (Center at $x=0, z=36$ ) ...... 90

27. Beam Location for Pelvic $44 \times 36 \mathrm{~cm}$ Field (Center at

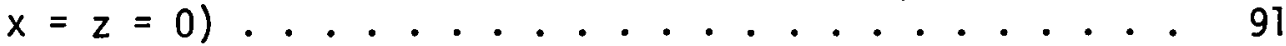

28. Beam Location for Chest Posterior-Anterior $36 \times 44 \mathrm{~cm}$ Field (Center at $x=0, z=53$ ) ......... 92

29. Effect of Gonad Shield on the Spectrum at the Testes -Pelvic Exam (Testes in the Beam) .......... 97

30. Effect of Gonad Shield on the Spectrum at the Testes -Abdominal Exam (Testes Near the Beam) ......... 
FIGURE

PAGE

31. Effect of Gonad Shield on the Spectrum at the Testes -Upper G.I. Exam (Testes Far from the Beam) . . . . . 99

32. Effect of Shielding with an Apron on the Spectrum at the Right Ovary -- Upper G.I. Exam -- 60 and 80 kVcp . . . 102

33. Effect of Shielding with an Apron on the Spectrum at the Right Ovary -- Upper G.I. Exam -- $100 \mathrm{kVcp}$...... 103

34. Effect of Shielding with an Apron on the Spectrum at the Right Ovary -- Chest PA Exam -- 60 and $80 \mathrm{kVcp}$.... . 104

35. Effect of Shielding with an Apron on the Spectrum at the Right Ovary -- Chest PA Exam -- $100 \mathrm{kVCp}$....... 105

36. Effect of Shielding with an Apron on the Spectrum at the Right Ovary -- Shoulder Exam -- 60 and $80 \mathrm{kVcp}$. . . . 106

37. Effect of Shielding with an Apron on the Spectrum at the Right Ovary -- Shoulder Exam -- $100 \mathrm{kVcp}$....... 107

38. Effect of Shielding and Field Size on the Spectrum at the Thyroid -- Shoulder Exam -- $60 \mathrm{kVcp}$...... 108

39. Effect of Shielding and Field Size on the Spectrum at the Thyroid -- Shoulder Exam -- $80 \mathrm{kVcp}$....... 109

40. Effect of Shielding and Field Size on the Spectrum at the Thyroid -- Shoulder Exam -- $100 \mathrm{kVcp}$....... 110

41. Spectrum as a Function of Depth -- $60 \mathrm{kVcp}$. . . . . . 112

42. Spectrum as a Function of Depth -- $80 \mathrm{kVcp} . . . . . .113$

43. Spectrum as a Function of Depth -- $100 \mathrm{kVcp}$. . . . . 114

44. Scattered Spectrum as a Function of Detector-FieldSeparation (DFS in Units of Centimeters) -- $80 \mathrm{kVcp}, .115$

45. Scattered Spectrum as a Function of Detector-FieldSeparation (DFS in Units of Centimeters) -- $80 \mathrm{kVcp}$. 116

46. Scattered Spectrum as a Function of Detector-FieldSeparation (DFS in Units of Centimeters) -- $100 \mathrm{kVcp} . .117$

47. Scattered Spectrum as a Function of Detector-FieldSeparation (DFS in Units of Centimeters) -- $100 \mathrm{kVcp} . .118$ 
FIGURE

PAGE

48. Spectrum at the Testes without Shield for Lower Spine Exam .................. 136

49. Spectrum at the Testes with Shield for Lower Spine Exam . . . . . . . . . . . . . . . . 137

50. Spectrum at the Testes without Shield for Full Spine Exam ........................ 138

51. Spectrum at the Testes with Shield for Full Spine Exam ...................... 139

52. Spectrum at the Testes without Shield for Abdominal Exam .................... 140

53. Spectrum at the Testes with Shield for Abdominal Exam. . 141

54. Spectrum at the Testes without Shield for Shoulder Exam ....................... 142

55. Spectrum at the Testes with Shield for Shoulder Exam . 143

56. Spectrum at the Testes without Shield for Upper G.I. Exam ....................... 144

57. Spectrum at the Testes without Shield for Upper G.I. Exam .................... 145

58. Spectrum at the Testes without Shield for Pelvic Exam. - 146

59. Spectrum at the Testes with Shield for Pelvic Exam . . 147

60. Spectrum at the Testes without Shield for Chest Exam . . 148

61. Spectrum at the Right Ovary for Lower Spine Exam . . . 149

62. Spectrum at the Right Ovary for Full Spine Exam . . . 150

63. Spectrum at the Right Ovary for Abdominal Exam . . . . 151

64. Spectrum at the Right Ovary for Shoulder Exam . . . . 152

65. Spectrum at the Right Ovary for Upper G.I. Exam . . . 153

66. Spectrum at the Right Ovary for Pelvic Exam . . . . . 154

67. Spectrum at the Right Ovary for Chest Exam . . . . . 155 
68. Spectrum at the Thyroid Gland for Lower Spine Exam . . . . 156

69. Spectrum at the Thyroid Gland for Full Spine Exam . . . 157

70. Spectrum at the Thyroid Gland for Abdominal Exam . . . . 158

71. Spectrum at the Thyroid Gland for Shoulder Exam . . . . 159

72. Spectrum at the Thyroid Gland for Upper G.I. Exam . . . 160

73. Spectrum at the Thyroid Gland for Pelvic Exam . . . . . 161

74. Spectrum at the Thyroid Gland for Chest Exam . . . . . . 162

75. Spectrum at the Heart Region for Lower Spine Exam . . . 163

76. Spectrum at the Heart Region for Full Spine Exam . . . . 164

77. Spectrum at the Heart Region for Abdominal Exam . . . 165

78. Spectrum at the Heart Region for Shoulder Exam . . . . . 166

79. Spectrum at the Heart Region for Upper G.I. Exam . . . . 167

80. Spectrum at the Heart Region for Pelvic Exam . . . . . 168

81. Spectrum at the Heart Region for Chest Exam . . . . . . 169

82. Spectrum at the Fifth I umhar Vertehra for Lower Spino

Exam ...................... 170

83. Spectrum at the Fifth Lumbar Vertebra for Full Spine Exam ...................... . 171

84. Spectrum at the Fifth Lumbar Vertebra for Abdominal Exam - 172

85. Spectrum at the Fifth Lumbar Vertebra for Shoulder Exam. - 173

86. Spectrum at the Fifth Lumbar Vertebra for Upper G.I. Exam ........................ 174

87. Spectrum at the Fifth Lumbar Vertebra for Pelvic Exam. . 175

80. Spectrum at the Fifth Lumbar Vertebra for Chest Exam . . . 176

89. Spectrum at the Thoracic Spine for Lower Spine Exam . . 177

90. Spectrum at the Thoracic Spine for Full Spine Exam . . . 178 
FIGURE

PAGE

91. Spectrum at the Thoracic Spine for Abdominal Exam . . . 179

92. Spectrum at the Thoracic Spine for Shoulder Exam . . . . 180

93. Spectrum at the Thoracic spine for Upper G.I. Exam . . . 181

94. Spectrum at the Thoracic Spine for Pelvic Exam . . . . 182

95. Spectrum at the Thoracic Spine for Chest Exam . . . . 183

96. Spectrum at the Eye Region for Lower Spine Exam . . . . 184

97. Spectrum at the Eye Region for Full Spine Exam . . . . . 185

98. Spectrum at the Eye Region for Abdominal Exam . . . . 186

99. Spectrum at the Eye Region for Shoulder Exam . . . . . 187

100. Spectrum at the Eye Region for Shoulder Exam (with $28 \times 44 \mathrm{~cm}$ Field) .............. 188

101. Spectrum at the Eye Region for Upper G. I. Exam . . . . 189

102. Spectrum at the Eye Region for Pelvic Exam . . . . . . 190

103. Spectrum at the Eye Region for Chest Exam . . . . . 191

104. Spectrum at the Testes as a Function of Field Size --

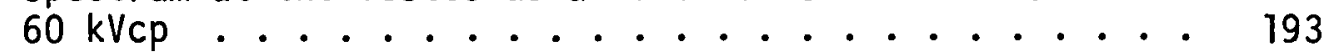

105. Spectrum at the Testes as a Function of Field Size --

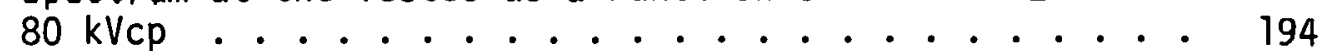

106. Spectrum at the Testes as a Function of Field S1ze -$100 \mathrm{kVcp}$................... 195

107. Spectrum at the Right Ovary as a Function of Field

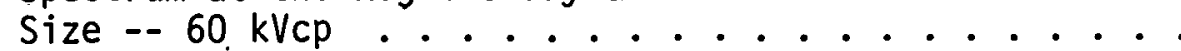

108. Spectrum at the Right Ovary as a Function of Field

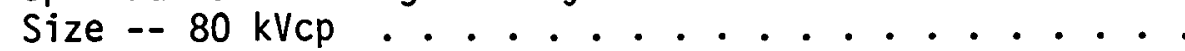

109. Spectrum at the Right Ovary as a runction of Field

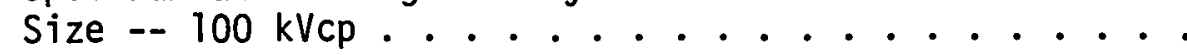

110. Spectrum at the Thyroid as a Function of Field Size - $60 \mathrm{kVcp}$................ 
FIGURE

111. Spectrum at the Thyroid as a Function of Field

Size $-80 \mathrm{kVcp}$. . . . . . . . . . . . . 200

112. Spectrum at the Thyroid as a Function of Field

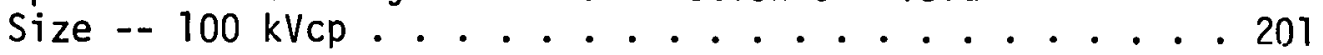

113. Spectrum at the Fifth Lumbar Vertebra as a Function of Field Size -- $60 \mathrm{kVcp}$. . . . . . . . . . . . . 202

114. Spectrum at the Fifth Lumbar Vertebra as a Function of Field Size -- $80 \mathrm{kVcp} \mathrm{..............} \mathrm{.} 203$

115. Spectrum at the Fifth Lumbar Vertebra as a Function of Field Size -- $100 \mathrm{kVcp}$............... . 204

116. Spectrum at the Thoracic Spine as a Function of Field Size - $60 \mathrm{kVcp}$. . . . . . . . . . . . . . 205

117. Spectrum at the Thoracic Spine as a Function of Field Size -- $80 \mathrm{kVcp}$.................. 206

118. Spectrum at the Thoracic Spine as a Function of Field Size -- $100 \mathrm{kVcp}$................... 207

119. Spectrum at the Eye Region as a Function of Field Size -- $60 \mathrm{kVcp}$. . . . . . . . . . . . . 208

120. Spectrum at the Eye Region as a Function of Field Size -- $80 \mathrm{kVcp}$. . . . . . . . . . . . . . 209

121. Spectrum at the Eye Region as a Function of Field

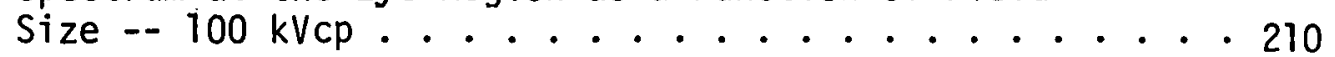


A program of measurements was made to determine the spectral fluence distributions at locations of significance in a heterogeneous, hominoid phantom exposed to $x$ rays in a manner simulating medical diagnostic radiology. The measurements were made with a specially constructed $\mathrm{NaI}(\mathrm{Tl})$ scintillation detector. The detector had a spherically shaped active volume $0.6 \mathrm{~cm}$ in diameter. The resolution of this detector was five times worse than that of a more conventional $\mathrm{NaI}(\mathrm{Tl})$ spectrometer. Resolution broadening and other distortions were removed from the observed pulse height spectra with a computer-coded, iterative unfolding technique. The performance of the spectrometer and the unfolding scheme was assessed by comparing, in a few cases, the unfolded $\mathrm{NaI}(\mathrm{Tl})$ spectra with spectra determined with a high resolution $\mathrm{Ge}(\mathrm{Li})$ spectrometer.

The measurements were made in a physical model of an idealized representation of an average adult patient. The phantom was constructed of molded lucite shells filled with differing materials to simulate lung, skeletal, and soft-tissue regions. To provide count rates suitable for scintillation spectrometry, a therapy-type $x$-ray machine was modified to operate at low exposure rates. However, because of the inherent filtration of the therapy-type tube, the output spectra of the $x$-ray machine were somewhat different than spectra typical of medical diagnostic radiology. How well the spectra of a particular $x$-ray machine resembles the spectra of the $x$-ray machine used in this study may influence the applicability of the data presented in this study. 
Spectral fluence distributions were determined at the testes, ovaries, thyroid, eye, heart, and two spine regions. $X$-ray beams simulating lower spine, full spine, abdominal, shoulder, upper gastrointestinal, pelvic, and chest examinations were employed at generating potentials of 60,80 , and $100 \mathrm{kVcp}$. The measured spectra were unfolded, and the results are presented graphically. The absorbed dose in softtissue was calculated from each spectrum, and these results are presented also. In addition, measurements were made to determine the effect of shielding on the spectrum and absorbed dose in regions of interest outside the useful beam.

The absorbed doses determined from spectral measurements were compared with a few ionization chamber measurements and with the results of Monte Carlo calculations. The absorbed doses determined with the spectrometer were generally lower than those determined with the ionization chamber, but the agreement in al1 cases studied was $\pm 16 \%$ or better. The absorbed doses determined at the testes, ovaries, and thyroid were compared with analogous calculations performed by the U.S. Bureau of Radiological Health. The agreement between the measured and calculated values was good when viewed in perspective with the inaccuracies in the experimental simulation, the differences between the experiments and the calculations, and the uncertainty in the Monte Carlo calculations due to poor statistics. For example, the calculations did not account for collimator scatter while the program of measurements demonstrated that collimator scatter contributed significantly to the absorbed dose to regions outside the useful beam. 
In addition to showing the feasibility and shortcomings of inphantom. spectrometry, the results of this study demonstrated the significance of collimator scatter. It is suggested that more study be given to this effect and to the benefit of shielding all body areas outside the useful beam. 


\section{CHAPTER I}

\section{INTRODUCTION}

In 1896 Wilhelm Conrad Roentgen made a public announcement of his discovery of $x$ rays. Besides reporting many of the physical properties of $x$ rays, he also showed that $x$ rays could be used to take pictures of the bones inside a human hand. From the period immediately following Roentgen's discovery until the present, $x$-ray photography or radiography has been a very valuable tool in the practice of medicine.

However, shortly after the announcement of the discovery of $x$ rays, there was evidence that $x$ rays could be harmful also. Grube, a man who had been manufacturing Crookes tubes and studying the fluorescence of chemicals before Roentgen's public announcement, developed erythema and finally ulceration on the back of his hand. He sought medical aid twenty-three days after Roentgen's announcement (Mo67). Three months later, Dr. J. Daniels of Vanderbilt reported that one of his colleagues had lost his hair following x-ray examination of his skull (E143). Fourteon months after Roentgen's announcement, 69 cases of skin damage due to $x$ rays were reported $(S c 97)$. Thus from its beginning, the use of $x$ rays on humans has had the potential to be both beneficial and harmful to mankind.

Today in this country, the largest use of $x$ rays or other ionizing radiation on humans is in the practice of medical diagnostic radiology. According to the estimates of the Advisory Committee on the Biological Effects of Ionizing Radiations (BEIR Committee) of the National Academy 
of Sciences, medical diagnostic use of $\mathrm{x}$ rays is by far the largest manmade source of radiation exposure to the U.S. population. Further it is estimated that medical diagnostic radiology comprises $40 \%$ of the total U.S. population's exposure to all sources of ionizing radiation.

Figure 1 shows a summary of the BEIR Committee's estimates for 1970 (BEIR72). Although actual whole-body doses for individuals and average whole body-doses for subsets of the population may vary greatly from the estimates of average whole-body doses presented, clearly medical diagnostic radiology is overwhelmingly the largest source of man-made radiation exposure and is definitely non-trivial in comparison to the U.S. population's total radiation exposure from all sources.

A major job of the health physicist is "...to prevent acute radiation effects and to limit the risk of late effects to an acceptable level (ICRP66)." In accomplishing this task, it is necessary that the health physicist quantify various aspects of radiation fields and relate his measurements to given radiation safety standards ( $R i 72$ ).

Any radiation field of one type (i.e., alpha, beta, neutron, photon, etc.) can be described by specifying its fluence distribution $\Phi(E, \hat{\Omega})$, where $\Phi(E, \hat{\Omega})$ is the number of particles of energy $E$ per unit energy and with direction $\hat{\Omega}$ per unit solid angle. If $\Phi(E, \hat{\Omega})$ for one type of radiation is known at all points of interest in a radiation field, then in principle any quantity can be calculated. However, the early $x$-ray and other radiation researchers had neither a comprehensive knowledge of the nature of radiation nor the electronic and solid-state technology necessary to make spectral measurements as we know them today. So it is not surprising that the ability of $x$ rays to ionize gases was the basis of the first widely accepted standard of radiation measurement. 


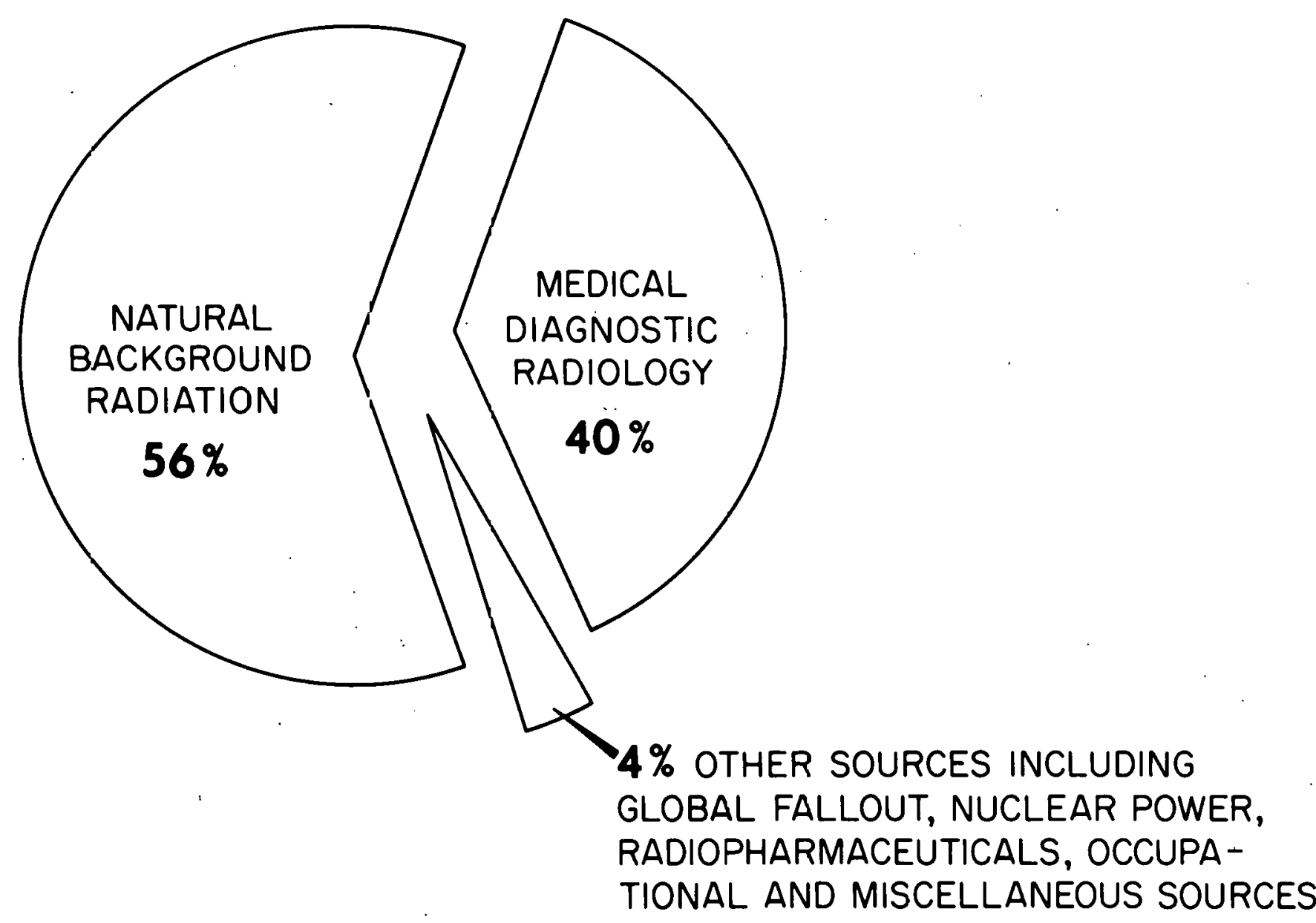

Figure 1. A Summary of BEIR Committee Estimates of Annual WholeBody Average Dose Rate in the United States in 1970 (BEIR72). 
The quantity, exposure, defined as the amount of ionization produced by electromagnetic radiation in equilibrium with its secondary radiation in a reference amount of air, was formalized by the International Commission on Radiation Units by its definition of the roentgen in 1928 (ICRU28). One of the earliest proposed radiation protection standards related biological effect to the exposure produced by an incident $x$-ray field (Mu25). As the energy of available $x$-ray beams increased and as other forms of radiation were discovered, it became obvious that quantifying a radiation field in terms of exposure, alone, was insufficient or inappropriate. When reporting $x$-ray exposure measurements it became common practice to include some information related to the energy spectrum, such as generating potential and filtration (Ri72). This technique of specifying the exposure together with limited spectral information is still used today by radiotherapists in calculating the internal distribution of energy deposited by external beams of photons (Jo69).

Today exposure is defined as

The quotient of dQ by dmi where dQ is the absolute value of the total charge of the ions of one sign produced in air when all the electrons liberated by photons in a volume element of air having mass $\mathrm{dm}$ are completely stopped in air (ICRU71).

Although this definition is the end product of several revisions made in order to remove ambiguities and to increase rigor, it describes essentially the same quantity that was defined in 1928. The special unit of exposure is the roentgen $(R)$, where $1 R$ is exactly equal to 2.58 $\times 10^{-4}$ coulombs per kilogram. 
Two other quantities are commonly used in quantifying aspects of a radiation field. with respect to risk. One is absorbed dose, which is defined as

The quotient of $d \bar{\varepsilon}$ by $d m$, where $d \bar{\varepsilon}$ is the mean energy imparted by ionizing radiation to the matter in a volume element and $\mathrm{dm}$ is the mass of the matter in that volume element (ICRU71).

The special unit of absorbed dose is the rad, where 1 rad equals $10^{-2}$ joules per kilogram (or 100 ergs per gram). The other quantity, specifically designed for radiation protection applications, is the dose equivalent, $H$, which is defined as,

The product of $D, Q$, and $N$, at the point of interest in tissue, where $D$ is the absorbed dose, $Q$ is the quality factor and $N$ is the product of any other modifying factors. The special unit of dose equivalent is the rem. When $D$ is expressed in rads, $H$ is in rems (ICRU71).

The quality factor is a weighting factor chosen, somewhat arbitrarily, to indicate the biological effectiveness of a given absorbed dose of different types of radiation. For $x$-radiation the quality factor is chosen to be unity. The factor, $N$, is to allow for the effects of the distribution of absorbed dose in space and time. For external sources this factor is chosen to be unity. Although the concept has been criticized for its lack of physical basis, its imprecision, and the difficulty of its determination, the dose equivalent is used in the most general expression of risk (Ri72; Th75).

For the purpose of having radiation protection units conform to the SI system of units, two other units have been introduced recently. The gray (Gy) is a unit of absorbed dose and is equal to 100 rads. The sievert (Sv) 
is a unit of dose equivalent and is equal to 100 rems. However, since there is some uncertainty regarding the general acceptance and use of these units in the U.S., they will not be used in this text.

It might also be noted that there is a loose correspondence between the exposure (measured in roentgens) of an x-ray field and the absorbed dose (measured in rads) to a small mass of muscle tissue placed in that field. The absorbed dose to muscle equals $95 \% \pm 3 \%$ of the exposure. This numerical correspondence is due to the size of the constants used in defining the units and to the fact that the propensities of air and muscle per unit weight to absorb energy from an $x$-ray beam do not vary much relative to each other over a wide range of $x$-ray energies. However, the absorbed dose in bone may be greater than four times the absorbed dose in muscle or the exposure in air at particular energies within the range typically used in medical diagnostic radiology (J069).

It is widely accepted,

$$
\begin{aligned}
& \text {... that in the vast majority of cases, exposure to } \\
& \text { ionizing radiation results in an increase in the en- } \\
& \text { tropy or disorganization of cell guidance provided } \\
& \text { by the vast library of information contained in the } \\
& \text { nucleus of a target cell, be it a somatic or germ } \\
& \text { cell. On this basis alone it is difficult to con- } \\
& \text { ceive of the existence of a threshold or safe level } \\
& \text { of exposure to ionizing radiation (Mo7l). }
\end{aligned}
$$

On the other hand, it is obvious that in many cases (but not all), the use of medical diagnostic $x$ rays provides great benefit to the patient. Estimates of risk are quantified in terms of the basic quantities and units outlined above. From the standpoint of decision making, it is unfortunate that there is no accepted method for quantifying estimates of benefit. 
In 1972 the BEIR Committee published a report on the effects of low levels of ionizing radiation. After a "review and re-evaluation of existing scientific knowledge concerning radiation exposure of human populations," the BEIR Committee arrived at some "best estimates" of radiation risks (BEIR72). Estimates of the long-term effect of exposure of the U.S. population such that the average dose equivalent rate from all sources was $170 \mathrm{mrem} /$ year included:

1. An incidence of $0.75 \%$ (per year) of serious genetic or partially genetic defects and

2. An increase of $5 \%$ in the 111 -health of the population (assuming that $20 \%$ of $i 11$-health is proportional to mutation rate, a 20 rem doubling dose, and a 5 rem per 30 year reproduction generation).

3. An increase of about $2 \%$ in the cancer death rate (about $0.3 \%$ in the overall death rate from all causes) (BEIR72).

Although the estimates of exposure from medical diagnostic $x$ rays and the estimates of effects of ionizing radition are filled with uncertainties, it is apparent that the effects are non-trivial. Morgan estimates that current medical diagnostic $x$-ray practice results in 4,100 to 78,000 deaths per year (Mo71). Unquestionably diagnostic radiology also saves many lives. The health physicist and the medical professional, although their manner and methods are different, are concerned with minimizing human suffering. The goal of reducing the adverse effects while maintaining or improving the quality of medical radiology should be actively pursued by both. Therefore it is not surprising that among the BEIR Committee recommendations are the following which concern, in whole or in part, medical diagnostic $x$-ray exposure:

1. No exposure to ionizing radiation should be permitted without the expectation of a commensurate benefit, 
2. The public must be protected from radiation but not to the extent that the degree of protection provided results in the substitution of a worse hazard for the radiation avoided.

3. Medical radiation exposure can and should be reduced considerably by limiting its use to clinically indicated procedures utilizing efficient exposure techniques and optimal operation of radiation equipment. Consideration should be given to the following:

1) Restriction of the use of radiation for public health survey purposes, unless there is a reasonable probability of significant detection of disease;

2) Inspection and licensing of radiation and ancillary equipment;

3) Appropriate training and certification of involved personnel. Gonad shielding (especially shielding of the testes) is strongly recommended as a simple and highly efficient way to reduce the Genetically Significant Dose.

4. Every effort should be made to assure accurate estimates and predictions of radiation equivalent dosages from all existing and planned sources (BEIR72).

Partly due to the recent criticisms of the traditional, but accepted, dosimetric quantities, a new approach to radiation dosimetry has been proposed (Hu74). In this system, time-dependent probabilities for a specific biological effect in a given species are operationally derived from basic aspects of the radiation field. In this formalism, the flux density, $\phi(E, \hat{\Omega}, \vec{r}, \tau)$, is the general description of a radiation field present in (or incident upon) a biological system or subsystem. The $E, \hat{\Omega}, \vec{r}, \tau$ indicate the energy, angular, positional, and time dependence of the field. $P_{i j}(t>\tau)$ is the probability of observing the biological effect $i$ in species $\underline{j}$ at any future time $t>\tau$. It is assumed that an operator exists such that:

$$
0_{i j} \phi(\tau)=P_{i j}(t>\tau)
$$

where the arguments $E, \hat{\Omega}, \vec{r}$ have been suppressed for convenience and where 
$0_{i j}$ is the operator which links the field with effect $\underline{i}$ in species $\underline{j}$. Assuming that $0_{i j}$ exists, equation $(1-1)$ is merely the mathematical expression of assuming the principle of cause and effect. In practice, the operator $0_{i j}$ might be very difficult to define accurately enough to make the solution of equation (1-1) feasible.

A set of measurements made in a radiation field can be expressed as $R(\varepsilon, \hat{\Omega}, \vec{r}, \tau)$ where $\varepsilon$ is the energy deposited in the detector and the other arguments are as before. For some detectors, i.e. spectrometers, it is possible to deduce $\phi$, the general description of the radiation field. That is there is an operator, $0_{d}$, characteristic of the detector, such that:

$$
0_{\mathrm{d}} R(\varepsilon, \hat{\Omega}, \vec{r}, \tau)=\phi(E, \hat{\Omega}, \vec{r}, \tau)
$$

is simply stating mathematically that the detector is a spectrometer.

Equation (1-2) implies that an unfolding procedure such that the spetral flux density distribution, $\phi$, can be deduced from the spectrometer measurements, R. Performing this unfolding with reasonable accuracy may be difficult or impossible. It often requires computer-programmed, iterative methods and may lead to answers with large uncertaintles (He74). Equation $(1-3)$ can be derived from equations (1-1) and (1-2):

$$
0_{i j} 0_{d} R(\varepsilon, \hat{\Omega}, \vec{r}, \tau)=0_{i j}^{-} R(\varepsilon, \hat{\Omega}, \vec{r}, \tau)=P_{i j}(t>\tau) .
$$

If the operator, $n_{i j}$, exists and can be adequately defined, then unfolding to find $\phi(E, \hat{\Omega}, \vec{r}, \tau)$ is not necessary. Further, in principle, $0_{i j}$ may be built into the electronics of a detector such that it reads probability of biological effect directly. Thus instead of using "rad-meters" or 
"rem-meters", health physicists might have "cancer-meters" or "sickness meters."

Hurst, Garrett, and Payne conclude their article by recommending that radiobiological experiments be conducted in such a manner that the time-dependence of particular effects can be inferred and that radiation protection instrumentation research should "concentrate more on those methods which can characterize the radiation field" (Hu74).

The objective of the research presented in this dissertation was the design, development, and application of a spectrometric dosimetry system for use in experiments simulating medical diagnostic $x$-ray exposure configurations. The applications of the system were to include:

1. A demonstration of the feasibility of spectrometric dosimetry measurements.

2. Determination of the values of various dosimetric parameters within an anthropomorphic phantom and comparison of these with values determined by other methods. 
CHAPTER II

THEORY

\section{Production of $x$ rays}

Energetic electrons colliding with atoms may generate $x$ rays in one of two manners. When a collision causes a vacancy to occur in an inner shell of an atom's electronic structure and when that vacancy is filled by an electron transition from a higher shell, an $x$ ray may be produced. The energy of the emitted photon is equal to the difference between binding energies of the initial and the final state of the electron undergoing the transition. Since the binding energies of the different levels are characteristic of each element, $x$ rays so produced have an energy which is determined by the atomic number of the radiating atom and the particular transition which occurred. Such $x$ rays are called characteristic $x$ rays.

An inner shell vacancy may also be filled by a nonradiative process. During the transition, the binding energy difference may be imparted to an outer shell electron, and thus no $x$ ray is produced. The ejected outer shell electron is called an Auger electron. The probability of $x$ rays being emitted from a vacancy in a particular shell is called the fluorescence yield (Ev 65).

Characteristic $x$ rays are labeled according to the initial and final states of the electron undergoing transition. $x$ rays originating from vacancies in the $K$ shell are called $K \times$ rays and are subscripted by $\alpha$ and $\beta$ to indicate that the electron undergoing transition originated in 
the $L$ or $M$ shell respectively. Further, numerical subscripts are used to indicate the particular sub-shell as needed. The energy of $k \times$ rays varies roughly as $(Z-1)^{2}$, (where $z=$ atomic number) and for example, the $K_{\alpha 1}$ $x$ ray of oxygen, sodium, iodine, tungsten, and lead are $0.52,1.04$, $28.6,59.3$, and $75.0 \mathrm{keV}$ respectively. The $\mathrm{K}$-shell fluorescence yield increases as a monotonic sigmoid with increasing atomic number. The $\mathrm{K}$-shell fluorescence yield for low $\mathrm{Z}$ elements, such as oxygen and sodium, are indeterminately small. Auger electron emission is the overwhelmingly preferred process for such elements to fill an inner shell vacancy. On the other hand the K-shell fluorescence yield for iodine, tungsten, and lead are $86 \%, 94 \%$, and $97 \%$ respectively (Le67).

Swift electrons may also create $x$ rays directly by inelastic collisions with atomic nuclei. As the incident electrons are deflected and decelerated by the Coulomb field of a nucleus, "braking radiation" or bremsstrahlung is emitted. For incident electrons with a given kinetic energy, $E_{\max }$, the energy fluence, $\psi(E)$, of the emitted bremsstrahlung, in any particular direction is constant for $E<E_{\max }$ and zero for $E>E_{\max }{ }^{1}$ Hence the number of photons of a given energy (i.e., fluence, $\Phi(E)$ ) varies inversely with that energy (Ev65).

A rigorous theoretical treatment of the production of bremsstrahlung by electrons incident on a single atom requires theory from quantum mechanics. The treatment of the production of bremsstrahlung in targets of finite thickness must include a description of the transport of swift electrons in the target material. Because this transport is, in

\footnotetext{
Energy fluence, $\psi(E)$, is defined as the product of the fluence, $\Phi(E)$, and the energy, $E$.
} 
general, so complex, an exact theoretical treatment of $x$-ray production in "thick" targets is impossible. However, a simplified description based on semi-empirical and numerical methods, has been developed, and produces results which are in agreement with data determined by direct experiment (Ev65). The energy fluence, $\psi$, as a function of photon energy, $E$, is described by

$$
d \Psi(E) \propto Z\left(E_{\max }-E\right) d E
$$

where $E_{\max }$ is the kinetic energy of the incident electrons and $Z$ the atomic number of the target. The shape of the energy fluence spectrum suggested by equation (1-1) is that of the sum of "thin" targets each being bombarded by electrons of decreasing kinetic energy (Ev65). Since, in practice, x-ray tubes must be enclosed, useful forms of equation (1-1) usually include a term which reflects the absorption of low energy photons by the tube housing. Then equation (1-1) becomes

$$
d \Psi(E) \propto Z\left(E_{\max }-E\right) \exp -\mu(E) d \quad d E
$$

where $\mu(E)$ is an attenuation coefficient for the inherent filtration of the $x$-ray tube envelope and $d$ is its thickness. The $\mu(E)$ for various materials can be approximated by

$$
\mu(E)=A+\frac{C}{E^{n}}
$$

where $n>2$ and $A, C$, and $n$ are positive and are empirically determined. Thus the exponential term in equation (2-2) is rapidly decreasing below a particular value of $E$ and causes the low energy end of the spectrum to have a "cutoff" (Jo75). 
In addition to producing $x$ rays, swift electrons produce much heat in an $x$-ray target via ionization and excitation interactions. The fraction, $f$, of total incident energy converted into bremsstrahlung in a thick target is approximately

$$
f=Z E\left(7 \times 10^{-7}\right)
$$

where $E$ has units of keV (Ev65). Tungsten, which has a high melting point and a large atomic number $(Z=74)$; is a common $x$-ray target material. According to equation (2-4) only $0.5 \%$ of the kinetic energy of $100 \mathrm{keV}$ electrons incident on a tungsten target is converted into $x$ rays. The other $99.5 \%$ is converted into heat in the target. Tungsten's high melting point protects against excessive target burnout.

\section{Interactions of $X$ Rays with Matter}

$X$ rays with energy in the range of 10 to $150 \mathrm{keV}$ interact with matter in three major ways. The processes are photoelectric absorption, Compton scattering, and Rayleigh scattering. All three are interactions between photons and atomic electrons. Although photon interactions with nucleons, electric fields, and meson fields of nucleons are possible in some cases, such interactions for typical $x$ rays are either energetically forbidden or insignificant in comparison with the electron-photon interactions. Pair production is an importani interaction for photuns with energies above $1.22 \mathrm{MeV}$ but is energetically forbidden in the entergy range typical of diagnostic $x$ rays.

In the photoelectric process an incident photon is completely absorbed by interacting with and ejecting one of the atom's orbital electrons. The kinetic energy of the ejected electron is almost entirely 
the energy of the photon less the binding energy of the ejected electron. Although the rest of the atom recoils slightly in order to satisfy the conservation of momentum requirements, it has little kinetic energy imparted to $i t$. The vacancy in the electronic structure of the atom is filled by a radiative or nonradiative process as discussed in the previous section.

Compton scattering is a process in which a photon is deflected by an atomic electron. It can be considered to be a two-body process, since in cases where the binding energy of the struck electron is significant, photoelectric absorption is an overwhelmingly more probable interaction (Ev65). In a Compton process the energy and momentum of the incident photon are partitioned between the deflected photon and the struck electron. Using the relativistic expressions for total energy and momentum, the following well-known equation can be derived.

$$
\frac{h v^{\prime}}{h v_{0}}=\frac{1}{1+\alpha(1-\cos \theta)}
$$

where $h v_{0}$ and $h v^{\prime}$ are the energies of the initial and scattered photon, $a=h v_{0} / m_{0} c^{2}$, and $\theta$ is the scattering angle of the photon. The maximum energy transferred, $T_{\max }$, occurs when $\theta=180$. The maximum fraction of energy transferred can be written as

$$
\frac{T_{\max }}{h \nu_{0}}=\frac{1}{1+(1 / 2 \alpha)}
$$

Thus a $10 \mathrm{keV}$ photon may lose only $4 \%$ of its energy in a Compton interaction, while a $100 \mathrm{keV}$ photon may lose $28 \%$ of its energy in single Compton scattering event. 
Rayleigh scattering is a two-body process in which a photon is deflected by an atom as a whole, producing neither atomic excitation nor ionization. Analyzing such a collision by requiring conservation of relativistic momentum and energy, yields an equation homologous to the Compton equations, (2-5) and (2-6), except that $\alpha=h \nu_{0} / M_{0} c^{2}$, where $M_{0}$ is the rest mass of the atom. Thus a $50 \mathrm{keV}$ photon incident on a hydrogen atom $\left(M_{0} \simeq 1837 \mathrm{~m}_{0}\right)$ could lose at most $5 \mathrm{eV}$ of its energy. More detailed analysis of the physics of Rayleigh scattering shows that Rayleigh scattering must be confined to small angles. For $100 \mathrm{keV}$ photons incident on aluminum, more than half of the radiation is scattered less than $15^{\circ}$. Thus Rayleigh scattering (or "coherent" scattering) removes negligible amounts of energy from a photon field (Ev65).

The quantification of the interaction of photons with matter in the simple case of a beam incident upon a șlab usually leads to an exponential type equation of the form

$$
A=A_{0} e^{-B x}
$$

Equations of the form of (2-7) are used to compute photon fluence and flux density, energy fluence and flux density, and energy deposition and interaction site distributions. The coefficients and parameters of equation (2-7) are adapted to the particular situation being studied and the definitions of such parameters are given in many locations (ICRU7); Ev65; Ro68a; Jo69; Mo67). Briefly, if A describes the photon fluence or flux density, $A_{0}$ is the quantity before attenuation, $B$ is the linear attenuation coefficient, $\mu$, and $x$ is the depth into the material. Alternatively, $B$ is $\mu$ divided by $\rho,(\rho=$ the density $)$, is expressed in 
terms of area per unit mass and is called a mass-attenuation coefficient. In this case the $x$ term is the product of the depth and the density and has units of mass per unit area. Other common coefficients and parameters are explained in Table 1.

There are several consequences of the fact that equations of the form of (2-7) are macroscopic descriptions of processes that occur between photons and electrons. First is the fact that interactions at the photon-electron level are independent (i.e., a photon may not interact photoelectrically and Compton scatter with the same atom). Thus the interaction coefficients can be broken down linearly, and the linear attenuation coefficient may be expressed as

$$
\mu=\tau+\sigma+n
$$

where $\tau, \sigma$, and $n$ are the partial attenuation coefficients for photoelectric absorption, Compton scattering, and coherent scattering respectively. Similarly if an absorber is made up of a number of elements of various atomic numbers, then the mass attenuation coefficient for the compound or mixture can be calculated by

$$
{\frac{\mu}{\rho_{\text {material }}}}=w_{1} \frac{\mu}{\rho_{1}}+w_{2} \frac{\mu}{\rho_{2}}+w_{3} \frac{\mu}{\rho_{3}}+\ldots
$$

where $w_{1}, w_{2}, w_{3}, \ldots$ are the fractional amounts by weight of each element, and the $(\mu / \rho)$ 's are the respective attenuation coefficients of the elements in the absorber.

A second consequence of the atomic scale of the interactions of radiation with matter is its statistical nature. Thus equations of the 
Table 1. Coefficient: and Faramsters of Exponential hittenuation Equations of the Form $A=A_{a} e^{-B x}$

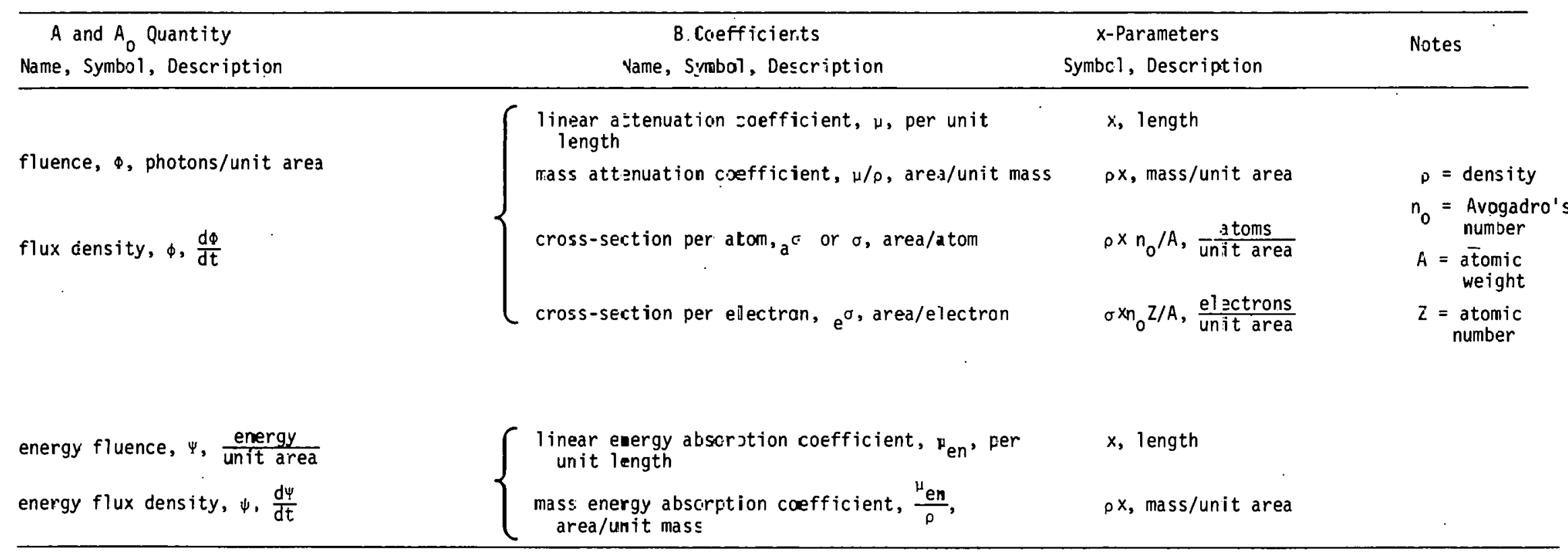


form of (2-7) only predict the expected values. The actual results of attenuation experiments will have a statistical variation which should follow Poisson statistics. The standard deviation of a set of measurements of fluence is estimated by the square root of the mean value. When large numbers of photons are encountered in radiation fields (e.g., $1 \mathrm{mR}$ corresponds to a fluence of $3 \times 10^{7}$ photons $/ \mathrm{cm}^{2}$ of $60 \mathrm{keV}$ photons), statistical uncertainties are small. However, the statistical nature of radiation interactions gives a probabilistic connotation to the coefficients in equations of the form of $(2-7)$. The magnitude of the cross section, $\sigma$ (area/atom as given in Table 1) is the probability that a photon incident upon a unit area containing one atom will interact with that atom. The mean range, $\lambda$, of a photon in a material can be computed by

$$
\lambda=\frac{\int_{0}^{\infty} x e^{-\mu x} d x}{\int_{0}^{\infty} e^{-\mu x} d x}=\frac{1}{\mu} .
$$

Similarly, the thickness of an absorber necessary to reduce the incident intensity of a beam to one half its original value (often called half value layer, H.V.L.) is defined as

$$
\text { H.V.L. }=\frac{\ln 2}{\mu}=\frac{0.693}{\mu} \text {. }
$$

For the purpose of discussion in this text, the description of the interactions of radiation with matter is limited to the photon energy range of 10 to $150 \mathrm{keV}$. The upper limit was chosen because common radiographic techniques do not employ generating potentials above $150 \mathrm{kV}$. A 
lower limit of $10 \mathrm{keV}$ was chosen because photons of this energy or lower have a mean free path in tissue which is very small compared with the dimensions of the human body and the dimensions of most human organs $(\lambda=0.16 \mathrm{~cm}$ in reference man soft tissue for $10 \mathrm{keV}$ photons). However, the low energy $(<10 \mathrm{keV}$ ) photon interactions in tissue would be of interest in the study of the mechanisms of radiation effects in biological material. Figure 2 shows the values of the mass energy absorption coefficient and partial mass attenuation coefficients in tissue as a function of photon energy. A computer-coded formulation of equation (2-9) incorporating the elemental composition of reference man soft tissue (ICRP75) and published values of the partial mass attenuation coefficients for the elements (Mc69) was used to calculate the coefficients presented (St74).

The data presented in Figure 2 shows the relative importance of photoelectric absorption and Compton scattering in soft tissue. For the purpose of discussion, the energy region from 10 to $150 \mathrm{keV}$ is subdivided into three regions. Above about $55 \mathrm{keV}$, the Comintun process is the most probable type of interaction in soft tissue and also is responsible for $50 \%$ or more of the energy absorption by soft tissue from all processes. (For $100 \mathrm{keV}$ photons Compton interactions in soft tissue are 57 times more probable than photoelectric interactions and account for $90 \%$ of the energy absorption.) Just below $30 \mathrm{keV}$ photoelectric absorption is the most probable type of interaction in soft tissue and is the dominant mode of energy absorption. In the middle region from 30 to $55 \mathrm{keV}$, the Compton interaction cross section in soft tissue is the largest, but photoelectric absorption is responsible for most of the energy absorption. 


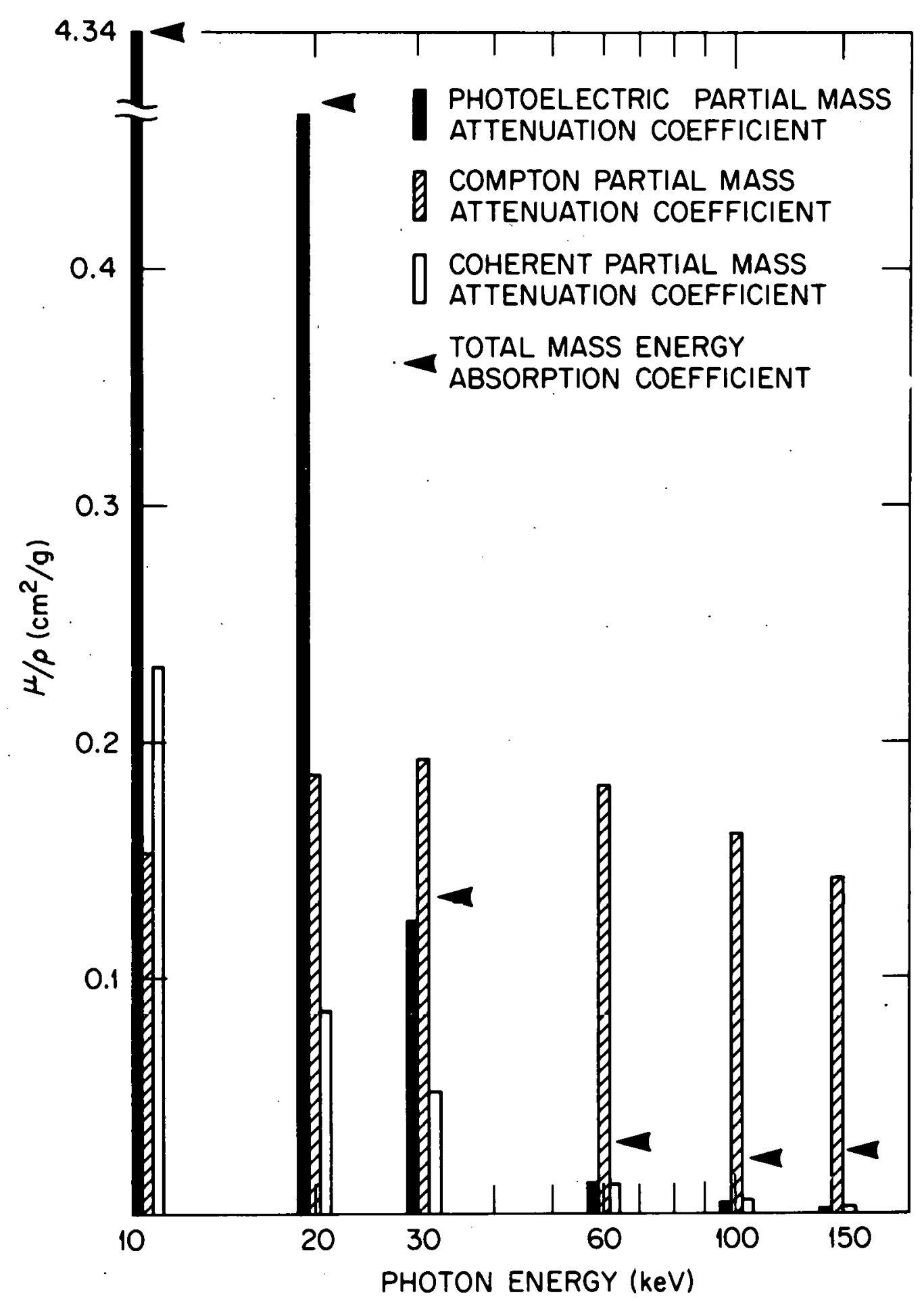

Figure 2. Mass Energy Absorption and Partial Mass Attenuation Coefficients for Snyder-Fisher Soft Tissue (St74). 
Coherent scattering usually is ignored, dismissed, or discussed very sketchily in most treatments of the interactions of radiation with matter (Ev65; Ev68, Fr66; Jo69, Mo67; Pr64). However, the magnitude of the coherent scattering cross-section in tissue is non-trivial compared with the magnitude of the Compton interaction cross-section for photons with energy between 10 and about $55 \mathrm{keV}$. The fact that coherent scattering causes negligible energy absorption in the energy range of interest usually is given as the reason for dismissing it from dosimetric discussions. Nevertheless, it is not obvious to this author that coherent scattering has an insignificant role in radiation transport, and the effect of coherent scattering is considered in the section of this text dealing with the tissue-equivalent design of the phantom.

\section{Monte Carlo Calculations}

In contrast to the simplicity of calculating the attenuation of a monoenergetic beam incident on a slab of matter, the solution of radiation transport equations for the scattering (including multiple scattering) and absorption of photons in complex and inhomogeneous geometry (i.e., a human body) is diffícult. In general, closed-form solutions are impossible. Monte Carlo calculations are one method of obtaining numerical estimates of the solutions of complex radiation transport problems. In such calculations, which in practice are usually done using a digital computer, a set of photon "histories" are generated one at a time, and the results are abstracted from the data provided by generation of the "histories." In the simplest form of such calculations, each photon "history" is started by assigning the photon an initial position, direction, and energy. The distance that a single photon will travel into a 
material before interacting is undeterminable. However, the probability distribution of the possible penetration distances is given by equations of the form of (2-7) with $x$ being the distance of penetration, $A_{0}=1$, and $B$ being the linear attenuation coefficient $(\mu)$. In a Monte Carlo calculation, a photon can be "transported" by selecting a penetration distance according to this probability distribution and calculating the coordinates of the first interaction site.

Since the probability distributions for absorption, scattering, and if applicable, scattering angle are known, the photon can be absorbed or scattered according to random selections from the appropriate probability distributions. Thus a photon can be followed from its source until it either is absorbed or escapes from the region of interest (Ro68b). As a set of photon histories is generated, mean values of the amount of energy deposited in various regions, the number of photons crossing a certain boundary, or other quantities of interest can be accumulated. These accumulated mean values are estimates of the true mean values.

According to sampling theory, statistical quantities such as variance and standard deviation can be used to estimate the reliability of their respective calculated sample means. The data needed to calculate the statistical quantities can be accumulated along with the mean value data.

One statistic often calculated is called the coefficient of variation. This quantity is the standard deviation divided by the mean. It is dimensionless and is usually expressed in percent. Snyder has suggested that estimates of absorbed dose determined by Monte Carlo calculations would be expected to vary about the true mean by as much as twice 
the product of the coefficient of variation times the mean $(S n 70)$. Thus an estimate provided by Monte Carlo calculation with a coefficient of variation in excess of $50 \%$ should be considered unreliable.

The drawback to Monte Carlo calculations is that although, in theory, the estimate of any mean value can be determined to any desired degree of reliability (by following a sufficient number of photons), in practice the number of histories that can be generated ( 1 imited by the amount of computer time available) often does not determine an estimate with desired reliability. The generation of 200,000 histories is not uncommon. More sophisticated Monte Carlo calculations use variance reduction techniques such as weighting, splitting, and Russian roulette, that, although not directly analogous to physical processes, are statistically sound and improve the reliability of the results (Ca75).

In 1976 the U.S. Bureau of Radiological Health completed a study of the absorbed dose to several organs from common diagnostic $x$-ray exposure situations (Ro76a; Ro76b). The basis of this study was a set of 2,716 Monte Carlo calculations, of 30,000 photon histories each. The calculations were performed using an idealized anthropomorphic model described in subsequent chapters in this text. Average absorbed doses were calculated for the thyroid, ovaries, testes, uterus, and active bone marrow regions. Because of the general nature of the model and because of the simplifying assumptions and methodology used to keep the number of calculations manageable, the results of the study were not intended to provide data pertaining to specific individuals. Rather, it was intended that the data be used "...to evaluate the effect on organ dose of changes in technical parameters in or among facilities" (Ro76b). 


\section{Phantom Measurements}

In addition to Monte Carlo calculations, it is possible to obtain data on the transport and absorption of an x-ray field in a material of complicated geometry by appropriate measurements. Obviously to determine the transport and absorption of radiation in humans by experiment, it is often necessary to use a substitute structure for reasons of safety, availability, and convenience. Traditionally, such a substitute is called a phantom. For satisfactory simulation it must scatter and absorb radiation in a manner similar to the biological material it represents.

Early researchers used hollow anatomical models filled with water as phantoms (Qu37). Spiers, in attempting to find a dry substitute for water, developed a method for evaluating the equivalence of one material with another (Sp43). He defined a quantity called effective atomic number as

$$
\bar{Z}=2.94 \sqrt{\sum A_{i} Z_{i}^{2.94}}
$$

where $A_{i}$ is the fraction of electrons for element $z_{i}$, and the summation is over all elements in the compound. The effective atomic number reflects the change in photoelectric cross-section with $Z$. Since Compton scattering cross-sections per electron change little for low $Z$ elements, materials with similar electron densities and with similar $\bar{Z}$ would interact with radiation fields in a similar manner.

In 1949, the development of a solid substitute for water was reported (Jo49). Using the above criteria to determine its equilvalence to water, a substance of $60.8 \%$ paraffin, $30.4 \%$ pulyethylene, $6.4 \% \mathrm{MgO}$, 
and $2.4 \% \mathrm{Ti}_{2}$ which was easy to mold and machine and remained homogeneous was made. The material was called "Mix D," and its water equivalence was verified by experiment. Rectangular and human-shaped Mix D phantoms were used to assess the gonad dose from medical diagnostic $x$ rays (St55; Ad57; E161; Yo67; Ta72). Data acquired from Mix D phantoms were used to assess the hazards to populations from medical $x$ rays by the British and Japanese government commissions (Gr60; Ja61).

Other water substitute phantoms were used also. Rectangular phantoms of "Pressdwood" (or Masonite) were used to estimate gonad doses (We57; Bi57; Ja67). Human-shaped pressdwood phantoms with a human skeleton inserted and with cork substituting for the lungs were used to measure bone marrow and gonad doses (La57; Ep67). Paraffin wax phantoms were used for similar measurements (Li58; Du68). All of these phantoms have the disadvantage that they are designed to be equivalent to water not tissue. For $x$ rays with energy from 15 to $100 \mathrm{keV}$ water has a $4 \% 1$ arger photoelectric partial mass attenuation coefficient and an $8 \%$ larger coherent scattering partial mass attenuation coefficient than reference man soft-tissue (St74). Yet for higher energy photons, for which Compton scattering is the predominant interaction, water and pressdwood are adequate and, their use is recommended (J069). This suitability of water phantoms for use in experiments involving photons with energy higher than that typical of diagnostic radiology is not surprising. In this higher energy range Compton scattering in soft tissue is overwhelmingly the most important interaction and Compton scattering cross-sections are directly proportional to electron density. Excepting hydrogen, most elements found in tissue $(Z<20)$ have a similar electron density since their ratio of atomic number to atomic 
weight is approximately constant $(Z / A \simeq 0.5)$. Thus for energies above approximately $150 \mathrm{keV}$, materials which have a density and hydrogen content similar to tissue will absorb and scatter radiation via the Compton process in a manner similar to tissue.

In contrast to the simplicity of tissue-equivalence for higher energies, the propensity of a material to scatter and absorb radiation with energy typical of diagnostic $x$ rays is a complicated function of elemental composition. Further since the mean free path for photons in the diagnostic energy range is shorter than for higher energy photons, the tissueequivalence of a substitute material is more important. For example, a beam of $300 \mathrm{keV}$ photons passing through $20 \mathrm{~cm}$ of soft-tissue $(\mu=0.111)$ would be attenuated to $11 \%$ of its original intensity. The same beam passing through $20 \mathrm{~cm}$ of a material with an attenuation coefficient $10 \%$ larger than soft-tissue would be attenuated to $9 \%$ of its original intensity. Thus an increase of $10 \%$ in attenuation coefficient causes a $20 \%$ reduction in transmitted intensity for $300 \mathrm{keV}$ photons incident on a $20 \mathrm{~cm}$ thick slab of soft-tissue. An analogous calculation for $30 \mathrm{keV}$ photons ( $\mu=0.316$ in soft tissue) shows that an increase of $10 \%$ in attenuation coefficient causes a reduction of almost a factor of two in transmitted intensity. Thus, for photons in the diagnostic energy range, a smal1 error in the tissue-equivalence may cause a large error in the absorbed dose measure at a point remote from the point of incidence of the beam.

In 1966 the Alderson human-shaped inhomogeneous phantom was developed and made commercially available (A166). Phantoms of this type are composed of a human skeleton imbedded in tissue-equivalent plastic. Lower density material for lung regions may be inserted optionally. The 
Alderson phantom is sliced horizontally in two $\mathrm{cm}$ thick sections and small holes are located in each section to accommodate thermoluminescent dosimetry chips or miniature ionization chambers. Its manufacturers claim it is "soft-tissue equivalent," but due to the proprietary status of the tissue-equivalent plastic used, it is difficult to obtain exact information on its composition. Further since each human skeleton is different, each phantom is different and it has been observed by this author that some of these phantoms have skeletons that are too small for the exterior contours of the encasing plastic. Models of this phantom have been used for dosimetric studies of diagnostic $x$ rays (Ha72, Va72).

In assessing the state of the art of in-phantom dosimetry studies of the absorbed dose to organs of radiological interest, two shortcomings should be noted. First, much of the research discussed in the preceding paragraphs has little bearing on the question of somatic risk. Five of the eleven studies cited (St55; Aj57; Li58; Du58; E161) presented measurements of gonad doses only. Four studies (La57, Ep61, Yo67, Ta72) presented some measurements of absorbed dose to bone marrow regions in addition to gonad doses, and one study (Ha72) presented bone marrow absorbed doses only. One study (Va72) employed $x$-ray film and thermoluminescent dosimeters "sandwiched" in between layers of a random phantom. However, the results presented in this study did little more than confirm the results of Epp et al (Ep6l). Thus a vast majority of the studies cited presented data for only a few organs. A complete study relevant to somatic risk would have to have data for all radiosensitive organs.

The second major criticism of previous in-phantom dosimetry concerns the methods and apparatus used. It was not until the late 1950's 
that the concept of absorbed dose was introduced and formalized officially. Because definitions were in a state of flux, it is often difficult to determine exactly what quantity was measured in studies performed during this period. Also, some phantoms used in previous research were designed to be water-equivalent, not tissue-equivalent. The Rando phantoms used in some studies had undocumented tissue equivalence and variable skeletal geometry. As was discussed previously, tissue equivalence may be extremely important when one material is substituted for another. Mismatches of $10 \%$ (causing some absorbed dose measurements to differ by a factor of two) for equivalent maerials for use in diagnostic radiology experiments are not inconceivable. Even ICRU muscle and reference man soft-tissue have massenergy absorption coefficients that differ by $7 \%$ for $30 \mathrm{keV}$ photons. Thus materials equivalent to one standard composition will not be equivalent to another. In summary, the U.S. Bureau of Radiological Health, in looking for data from experiments with which to verify their calculations found that "few experimental data that have been obtained in a scientifically controlled manner are available with which to compare..." (Ro76).

Spectrometric studies using phantoms have been of several types. In 1951 a study of the spectral distribution of scattered and unscattered radiation in a paraffin block phantom was made (Gr51). Using the data of other researchers, the change of exposure measured with a spherical air-equivalent ionization chamber surrounded with layers of spherical copper shells of various thicknesses was examined. Then the exposure rate as a function of wavelength for unscattered and scattered radiation at the surface and $10 \mathrm{~cm}$ deep in the block was computed. Obviously, this early work is of an approximate and preliminary nature. 
In 1960 and 1967 similar experiments were performed in which a cylindrical tank of water was irradiated with a plane beam (Ma60; Ep67). A hollow lucite type was used to "extract" the spectrum of $x$ rays from the center of the tank and transmit it to an external $\mathrm{NaI}(\mathrm{Tl})$ scintillation spectrometer. In both experiments the entire apparatus could be rotated about the axis of the cylinder so that the spectrum of $x$ rays traveling in any direction could be measured. Since the tank was positioned horizontally, it could be filled to any desired level and the spectrum could be measured at various depths from the surface. Both researchers observed that the unscattered (or primary) and the scattered spectra became "harder" (i.e., had a higher average energy) with depth, and as expected for incident spectra typical of medical diagnostic $x$ rays, the scattered $x$-ray spectrum was "softer" than the primary. These experiments are the only reported attempts to measure the spectral fluence distribution inside a phantom exposed to medical diagnostic $x$ rays.

Several measurements have been made of the $x$-ray field scattered from a phantom exposed to $x$-ray beams $(\operatorname{Tr} 72$, Wa73, Str75). However, the purpose of these studies was to determine the half value layer or the average energy of the scattered radiation in order to design secondary shielding optimally. Their results are not relevant to the question of the spectrum inside a phantom exposed to diagnostic $x$-ray beams.

\section{Sodium Iodide Scintillation Spectrometry}

of the many methods for measuring the spectral fluence of an $x$-ray field, sodium iodide scintillation spectrometry was chosen as the method for use in this project. Despite its inferior resolution, scintillation 
spectrometry was selected because it allowed a wide latitude in choice of detector size and shape. Sodium iodide was chosen, in particular, because of its high intrinsic efficiency.

The scintillation process in sodium iodide activated with thallium can be described in three steps. An energetic electron set in motion by an $x$-ray interaction moves through the crystal until it dissipates all of its energy by ionizing and exciting other electrons. Some of these electrons (and their resultant holes) migrate to the thallium impurity centers. Some of these excited impurity centers de-excite in a radiative process. Thus, if the physics is understood (or if the end result is known as a function of the initial conditions), the light emitted from the scintillator in a particular band of wavelengths can be related to the kinetic energy of the energetic electron and, ultimately, the $x$ ray which initiated the process. For $\mathrm{NaI}(\mathrm{T} \ell)$ the emission band is centered around $420 \mathrm{~nm}$ and the decay time for the scintillation process is about $3 \times 10^{-7} \mathrm{sec}$. Further the light production in $\mathrm{NaI}(\mathrm{Tl})$ is linear with respect to initial electron energy for the range of energies of interest to spectrometry of $x$ rays (Pr64).

To complete the scintillation spectrometry process it is necessary only to collect the light produced by the scintillator, measure it, and allow for distortions and fluctuations that may occur. A light pipe in optical contact with the crystal is used to bring the light (or at least a fraction of the light) to the photocathode of a photomultiplier tube. The light impinging on the photocathode produces a pulse of electrons. This original pulse is amplified by an electron multiplication process in the photomultiplier tube. A preamplifier is used to convert the pulse of 
charge produced by the photomultiplier tube into a voltage pulse. The voltage pulse can be shaped and amplified by appropriate electronic circuitry. An analog-to-digital converter is used to digitize the magnitude of the voltage pulse. Thus a spectrum of $x$ rays incident upon the scintillator will produce a spectrum of pulse heights. A multi-channel analyzer is used to display and record the pulse height spectrum.

A measured pulse height spectrum can be related to the spectrum of incident $x$ rays by the operator equation

$$
R(N, \delta)=O_{d} \phi(E, \hat{\Omega}, \vec{r}, t)
$$

where $\phi(E, \hat{\Omega}, \vec{r}, t)$ is the spectrum incident on the detector, $R(N, \delta)$ is the response of the detector system, and $0_{d}$ is the operator, characteristic of the detector system, which relates the two. $R(N, \delta)$ is a collection pulse height spectra with $N$ being channel number, and $\delta$ is a dummy variable to indicate that for a given incident $x$-ray spectrum, a distribution of pulse height spectra would be expected. Equation $(2-13)$ can be simplified if certain conditions are met. If the rate at which $x$ rays interact with the scintillator is slow compared with the rate at which the spectrometry system can measure and catalogue the energy of the $x$ rays, the time dependence of equation $(2-13)$ can be ignored, and the spectral flux density can be replaced by the spectral fluence. Similarly if the spatial and angular dependence of the spectral fluence with respect to the detector are constant or if the response of the detector is independent of the angular and spatial distribution of the field incident upon the detector, these variables can be ignored. In this case equation (2-13) simplifies to 


$$
R(N, \delta)=0_{d} \phi(E)
$$

Obviously, for an ideal detector, $0_{d}=1$ and the inversion of equation $(2-14)$ is trivial. However, real scintillators have distortions and uncertainties which make both $0_{d}$ and its inverse more complicated.

The operator, $0_{d}$, must account for the difference between the incident $x$-ray spectrum and the spectrum of energy deposited in the active volume of the crystal. Further, $0_{d}$ must account for any nonlinearities in the scintillator's conversion of ionization and excitation into scintillated light and for any nonuniformity in the collection of such light. Finally, $0_{d}$, must incorporate the statistical nature of the photomultiplier's performance. Typically, for an input pulse of light falling uniformly on the photocathode, the electronic output pulse will be any one of a distribution of output pulses. The distribution of output pulses is Gaussian with both the mean and the variance of the distribution being proportional to the intensity of the light pulse.

To do spectrometry, equation (2-14) is not important but rather its inverse

$$
\phi(E)=0_{d}^{-1} R(N, \delta)
$$

is the equation which must be solved. It is agreed (He74; Wa72) that equation $(2-15)$ is 111 -conditioned and the direct inversion of the operator, ${ }_{d}$, to find $0_{d}^{-1}$ is not feasible. Further, by a series of measurements it is impossible to determine $R(N, \delta)$, the entire distribution of possible pulse height spectra. Therefore, the unfolding "problem" is to determine approximately the incident $x$-ray spectrum given one or a few pulse height spectra. 
The method used to deal with the unfolding problem in this work is an iterative, numerical method. As will be discussed in Chapter III, the response of the detector was determined for uniformly incident monoenergetic beams of photons. The response functions $R_{E_{1}}(N), R_{E_{2}}(N)$, $R_{E_{3}}(N), \ldots$, were determined for $E_{1}=10,12,14, \ldots, 130 \mathrm{keV}$. Then for an observed pulse height spectrum, $R(N)$, a suitable linear combination was found such that

$$
\left|A_{1} R_{E_{1}}(N)+A_{2} R_{E_{2}}(N)+A_{3} R_{E_{3}}+\ldots-R(N)\right| \leq G(N)
$$

where $G(N)$ is an acceptable error distribution. A computer-coded, iterative successive approximation technique was used to select the appropriate linear combination (Mc72). The details of the unfolding process and the determination of $G(N)$, the acceptable error distribution are discussed in chapter III. Obviously

$$
\phi(E)=A_{1} E_{1}+A_{2} E_{2}+A_{3} E_{3}+\ldots
$$

is an approximate solution of equation $(2-14)$. 
CHAPTER II I

\section{APPARATUS AND PROCEDURES}

An x-ray facility, a spectrometer, and a phantom were necessary to perform the in-phantom spectrometry measurements in a manner which simulated diagnostic medical radiology. Also needed were the electronics ancillary to the spectrometer and a computer-coded unfolding procedure.

\section{X-Ray Facility}

It was deemed necessary that the x-ray facility meet the following criteria:

1. Size. The shielded enclosure had to be large enough so that spectrometry measurements could be made with minimum scatter from shielding walls.

2. Wide range of operating capabilities. To facilitate collection of spectrometry data when the point of measurement was far from the incident beam and the intensity of the $x$-ray field was greatly reduced, the $x$-ray machine had to be capable of sustained output over periods on the order of 1000 seconds. To be able to collect data with the spectrometer actually in the incident beam, the $x$-ray machine had to be capable of operating at tube currents on the order of $1 \mu \mathrm{A}$.

3. Simulation of diagnostic $x$-rays. The $x$-ray machine had to have characteristics, such as inherent filtration and range of generating potentials, similar to those typical of diagnostic $x$-ray machines. 
4. Stability and regulation. The $x$-ray machine had to have good voltage regulation for the wide variations in tube current. It had to be a constant potential type machine with minimal high voltage ripple to ensure that the high voltage wave form had little variation over the wide range of tube currents. The $x$-ray machine output had to be stable and reproducible. Since an $x$-ray facility meeting the above criteria was not available, it was necessary to design and construct one. Figure 3 shows the layout of the facility, the shielding necessary, and some of the features of the safety interlock system. It was felt that neither a conventional diagnostic nor a conventional therapy $x$-ray machine would provide adequate stability and the wide range of operating capabilities needed. In general diagnostic $x$-ray machines are designed to operate at high tube currents (on the order of hundreds of milliamps) for short exposure times. Therapy $x$-ray machines are designed to operate continuously but with tube currents between 1 and 20 mA. Therefore, a cathode-near-ground x-ray machinc configuration was devised (see Figure 4), and a therapy-type $x$-ray machine was modified extensively to conform to this design. The cathode-nearground configuration is opposite from the cunveintional anode-grounded scheme employed in many diagnostic $x$-ray machines.

The $x$-ray tube, tube housing, supporting stand, collimator, and oil cooler were parts of a Siemens $250 \mathrm{kV}$ therapy $x$-ray machine. A therapytype-tube was chosen so that the $x$-ray machine could be operated continuously for as long as necessary to measure the spectra at locations remote from the incident beam. However instead of being operated in the bi-polar mode, the anode was operated at positive voltage, and the cathode was operated at near-ground potential. 


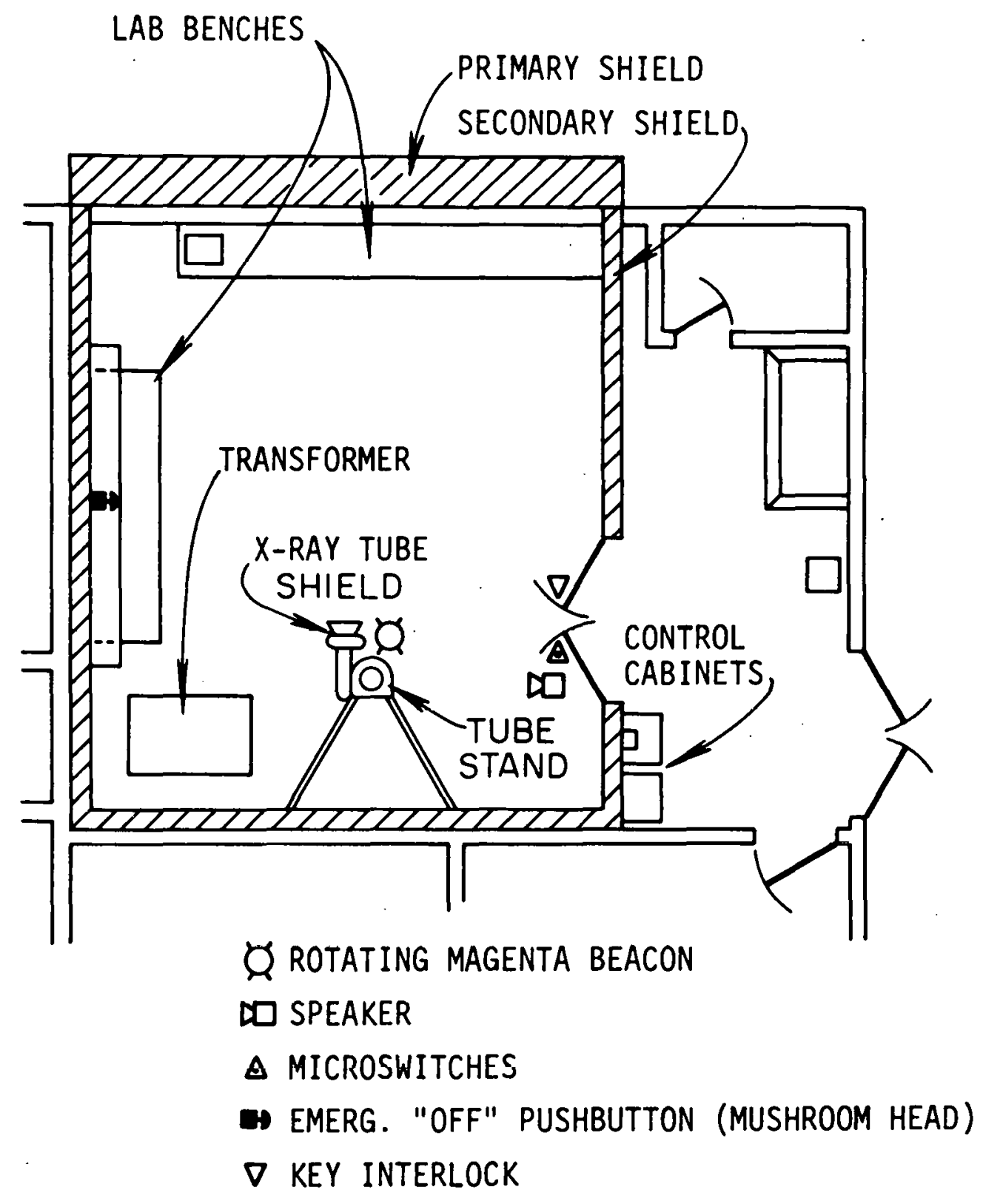

INTERIOR $20^{\prime} \times 20^{\prime}$

$\left.\begin{array}{l}\text { PRIMARY SHIELD 16" CONCRETE } \\ \text { SECONDARY SHIELD } 8 \text { ". CONCRETE OR } 1 / 8 \text { " Pb }\end{array}\right\} \times 8^{\prime} \mathrm{HIGH}$

Figure 3. Floor Plan for the X-Ray Facility. 


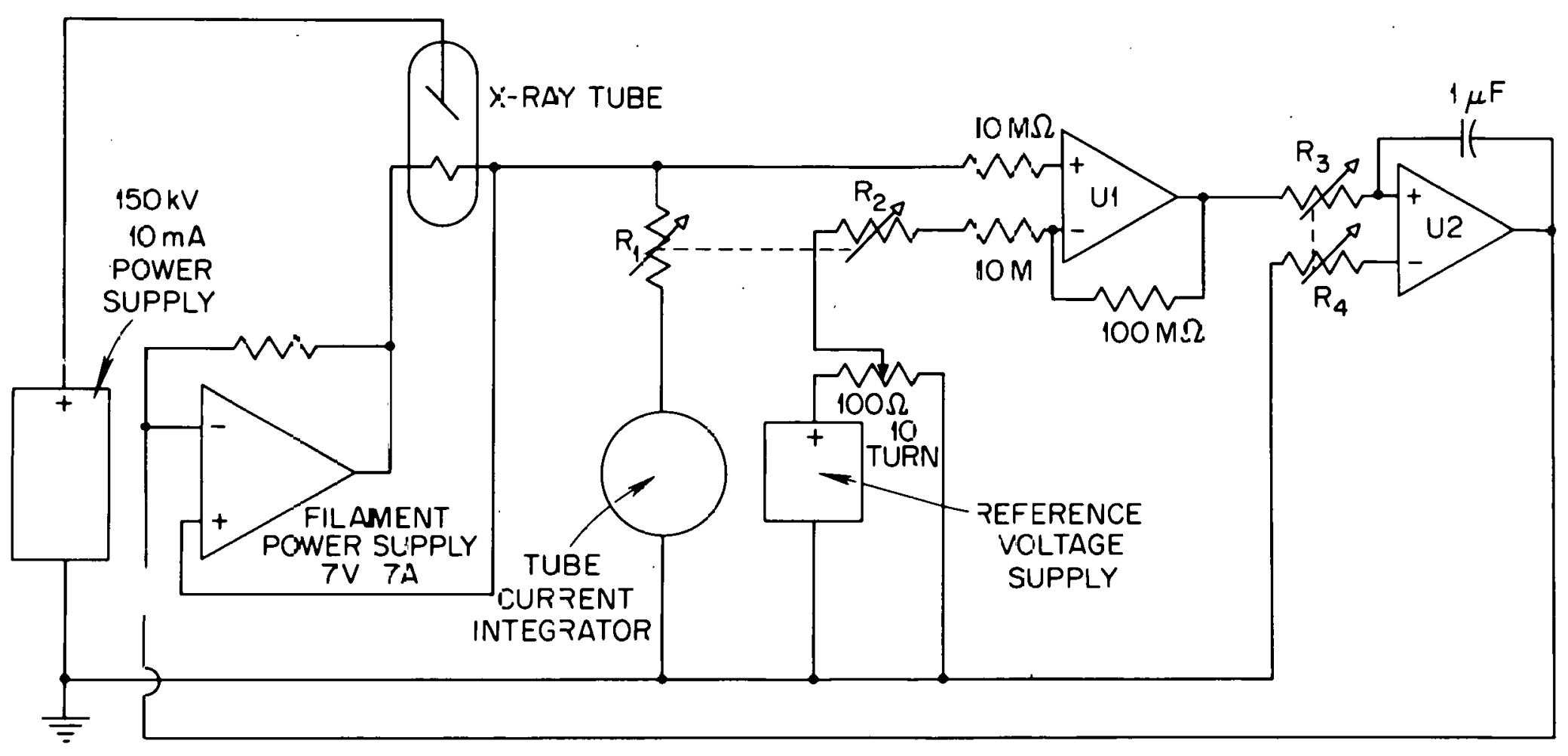

Figure 4. X-Ray Machine Schematic 
The positive high voltage was provided by a vacuum-tube regulated supply capable of $20 \mathrm{~mA}$ up to $75 \mathrm{kV}$ and $10 \mathrm{~mA}$ up to $150 \mathrm{kV}$. In this commercially available supply, an internal resistance chain provided feedback such that the voltage drop through the vacuum tube was continuously adjusted to provide a constant potential output. After procurement of the voltage supply, it was tested to ensure that it met the contracted specifications. During this testing the high voltage components were suspended in air, and the supply was operated at voltages up to $40 \mathrm{kV}$. A series of high voltage resistors were used to provide a test load that would draw approximately $3 \mathrm{~mA}$ at $40 \mathrm{kV}$. An oscilloscope was used to observe the shape of the high voltage waveform. The rootmean-square value of the ripple in the high voltage waveform at $40 \mathrm{kV}$ was observed to be approximately $0.05 \%$. Later, the output voltage, digitally selectable, was measured with a 1000 megohm, temperature-compensated voltage divider in parallel with the $x$-ray tube. The voltage divider was calibrated to an accuracy of $1 \%$. The selected output voltage agreed with the measured voltage within the $1 \%$ accuracy of the voltage divider. This agreement was checked for all operating voltages used in subsequent experiments and for currents from full load to the current drawn by the voltage divider (nominally $0.1 \mathrm{~mA}$ ). An increase in output voltage of at most $0.3 \%$ was observed when the tube current was varied from full load $(10 \mathrm{~mA})$ to minimum load $(1 \mu \mathrm{A})$.

The circuitry to control the tube current is pictured schematically in Figure 4. Operational amplifier Ul controls the filament current power supply by comparing the voltage developed by the tube current passing through $\mathrm{Rl}$ with the voltage provided by the reference voltage 
supply. Operational amplifier U2, its feedback capacitor, and input resistors prevent the relatively fast operational amplifier circuitry from over-regulating the slower filament. Typically time constants from one to ten seconds were necessary to prevent tube current oscillations. The integrator measures the tube current and serves as a timer via its trip control.

The output of the $x$-ray machine was calibrated with a Victoreen Radocon III ionization chamber and electrometer dosimetry system. The two chambers employed for these measurements had volumes of $32.6 \mathrm{~cm}^{3}$ and $326 \mathrm{~cm}^{3}$, respectively. The dosimetry system including probes was calibrated by the manufacturer with filtered $x$-ray beams with effective energies of $15,21,32,34.5,42,50,64,84$, and $140 \mathrm{keV}$. The measured exposure rate as a function of generating potential is shown in Figure 5 . It is a rule of thumb that the exposure rate of a diagnostic $x$-ray machine should vary proportionally with the square of the generating potential (Ch70). The reference line with a slope of 2.0 in Figure 5 shows that the exposure rate of the $x$-ray machine follows this rule. Also the exposure rate was measured over the entire range of tube current (see Figure 6). At a given generating potential the variation of exposure rate per unit tube current was generally less than the $\pm 2 \%$ accuracy claimed for the Radocon III. The horizontal dashed lines in Figure 6 are not "best fit" but are presented for reference only.

It was necessary to use the $32.6 \mathrm{~cm}^{3}$ chamber for tube currents above $3 \mathrm{~mA}$ and the $326 \mathrm{~cm}^{3}$ chamber for tube currents of $3 \mathrm{~mA}$ and below. At $3 \mathrm{~mA}$ the $32.6 \mathrm{~cm}^{3}$ chamber measured $1 \%$ higher than the $326 \mathrm{~cm}^{3}$ chamber. Thus it is presumed that the indicated increases in exposure rate 


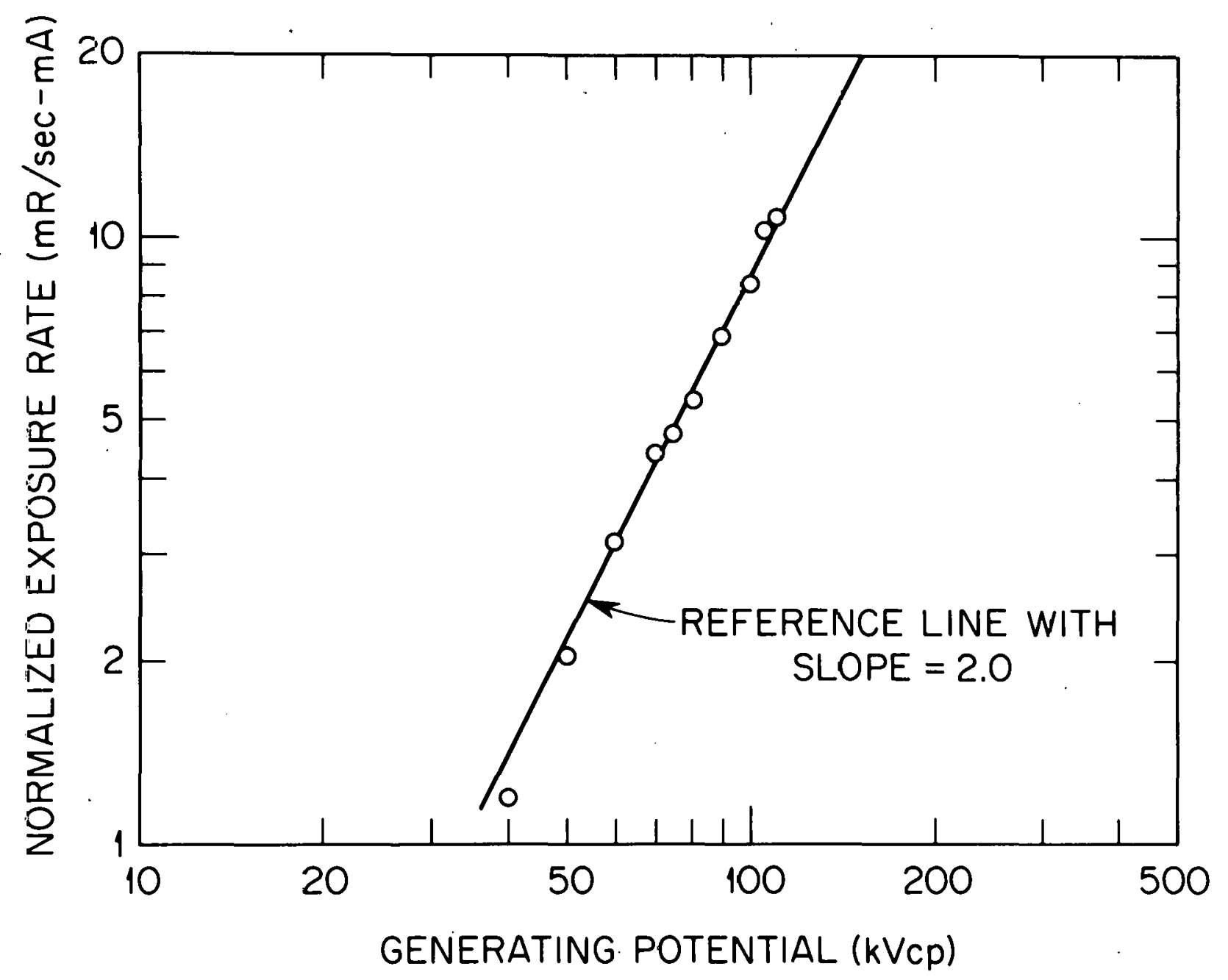

Figure 5. Exposure Rate at a Target to Detector Distance of $100 \mathrm{~cm}$ as a Function of Generating Potential. 


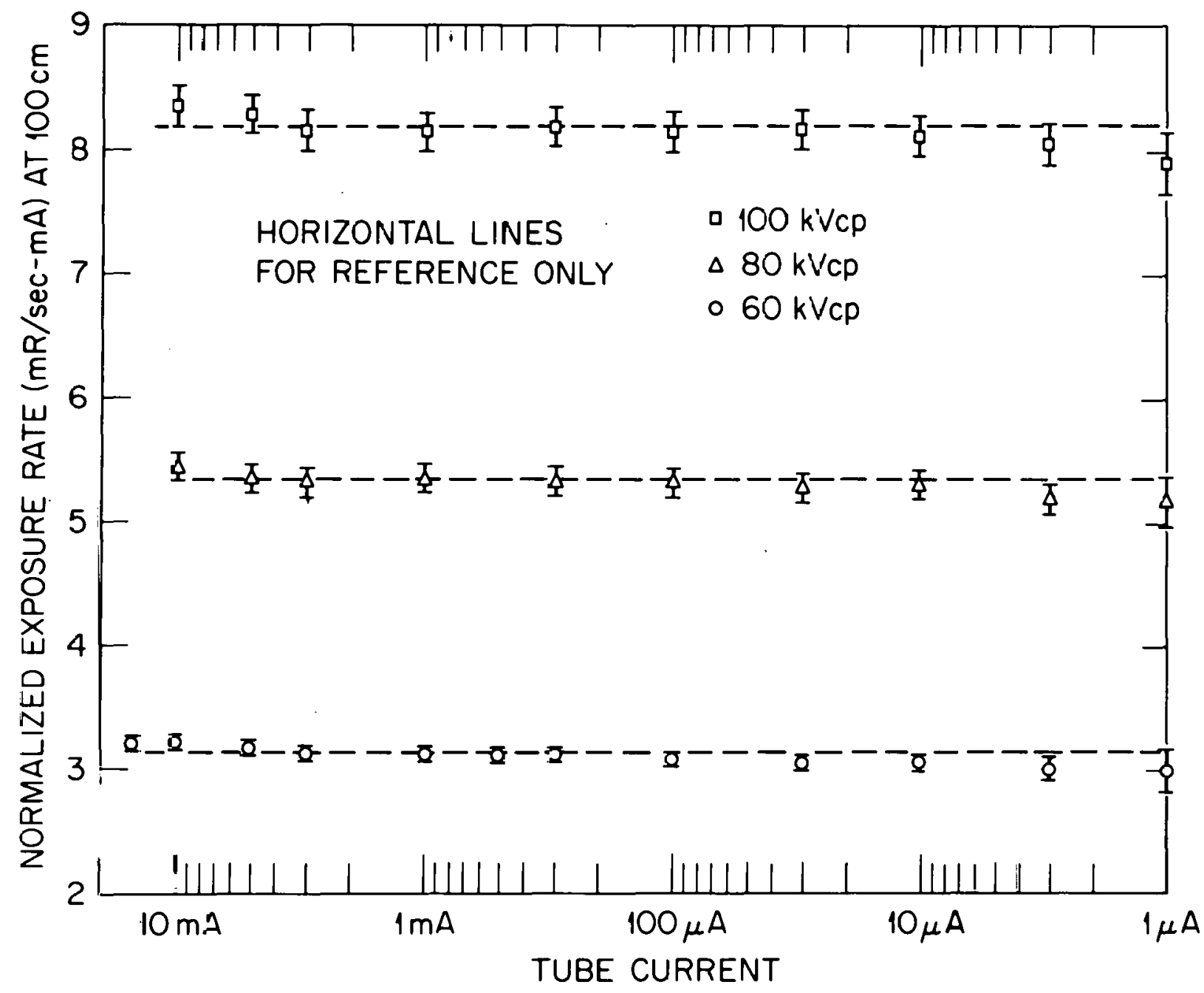

Figure 6. Normalized Exposure Rate as a Function of Tube Current (Error Bars Correspond to $\pm 2 \%$ Plus the Least Significant Digit Uncertainty). 
measured for tube currents from 5 to $15 \mathrm{~mA}$ are merely artifacts due to the difference in sensitivity of the chambers. It is not known whether indicated decreases in exposure rate measured for tube currents below $10 \mu A$ are due to a change in sensitivity of the dosimetry system or are due to an actual decrease in the output of the $x$-ray machine. Nevertheless, since all variations are less than $\pm 3 \%$, it was decided to treat all subsequent data as if the output of the $x$-ray machine as a function of tube current was essentially constant.

The spectral output of the $x$-ray machine was measured using a $\mathrm{Ge}(\mathrm{L} i)$ spectrometer. The spectrometer had an active depth of $7.1 \mathrm{~mm}$ and a $0.25 \mathrm{~mm}$ beryllium window. The resolution of the $\mathrm{Ge}(\mathrm{L} i)$ spectrometer was determined to be $0.9 \mathrm{keV}$ full-width at half-maximum (FWHM) for a $60 \mathrm{keV}$ photon $\left({ }^{241} \mathrm{Am}\right)$. Since the magnitude of the resolution was small compared with the width of the spectra to be measured, it was felt that no corrections for the Gaussian broadening of the Ge(Li) spectrometer data were necessary. However it was advised that the counting rate at which the spectrometer was used be kept less than 1,000 counts per second to avoid degrading the resolution (Be76). Several steps were taken to $l$ imit the counting rate. The spectrometer was positioned $3 \mathrm{~m}$ from the $x$-ray tube target, and a collimator with an aperture of $7.7 \times 10^{-3}$ $\mathrm{cm}^{2}$ was placed directly in front of the spectrometer. Spectra were measured for 60,80 , and $100 \mathrm{kVCp}$ generating potential with tube currents of 25,10 , and $6 \mu \mathrm{A}$, respectively. ${ }^{2}$ These measured spectra are presented graphically in Figures 7,8 , and 9 . The raw data spectra shown are the ${ }^{2}$ The $c p$ in $k V c p$ denotes the constant potential nature of the generating potential. 


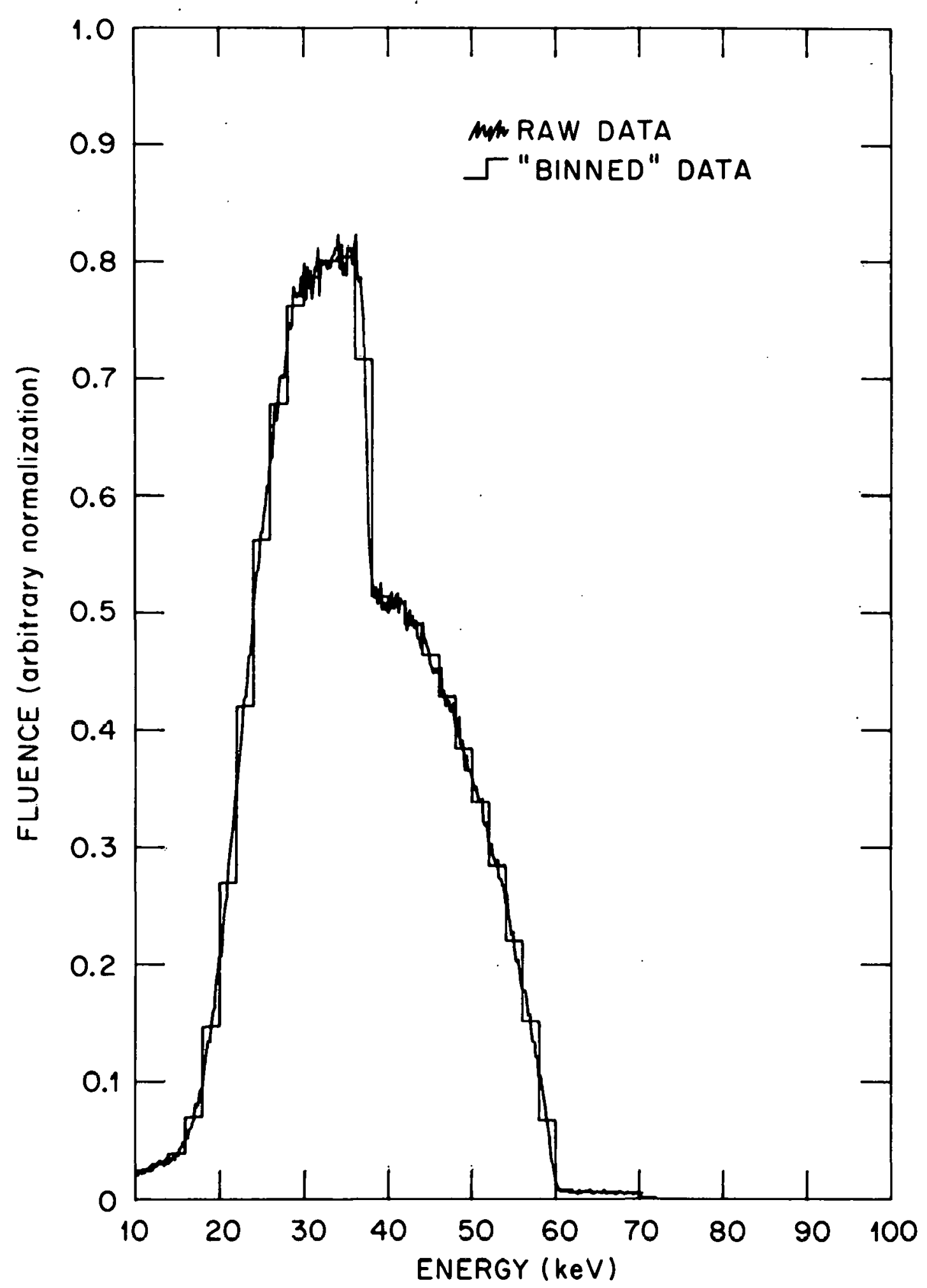

Figure 7. X-Ray Machine Output Spectrum Measured with a Ge(Li) Detector -- $60 \mathrm{kVcp}$. 


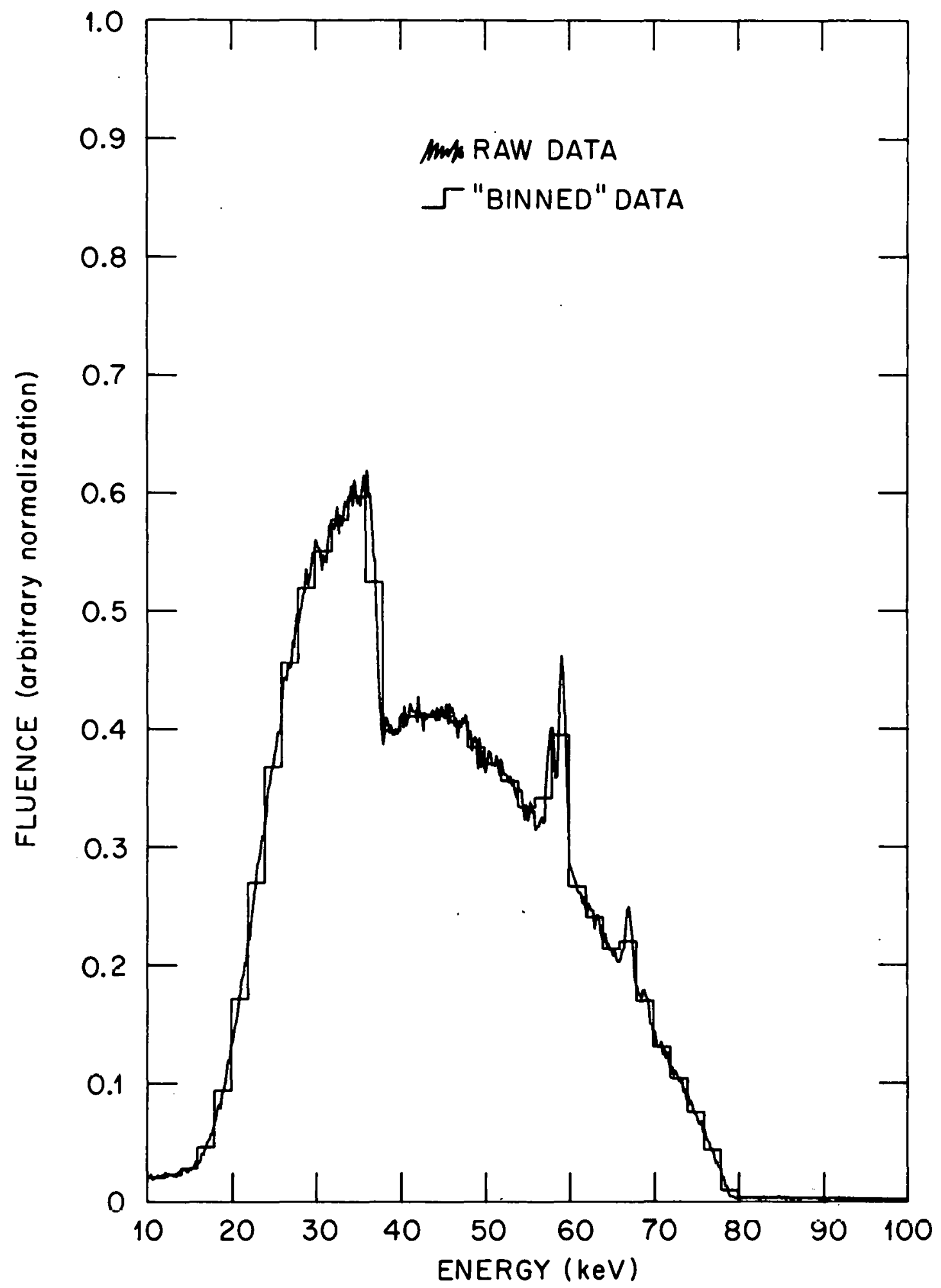

Figure 8. X-Ray Machine Output Spectrum Measured with a Ge(Li) Detector -- $80 \mathrm{kVcp}$. 


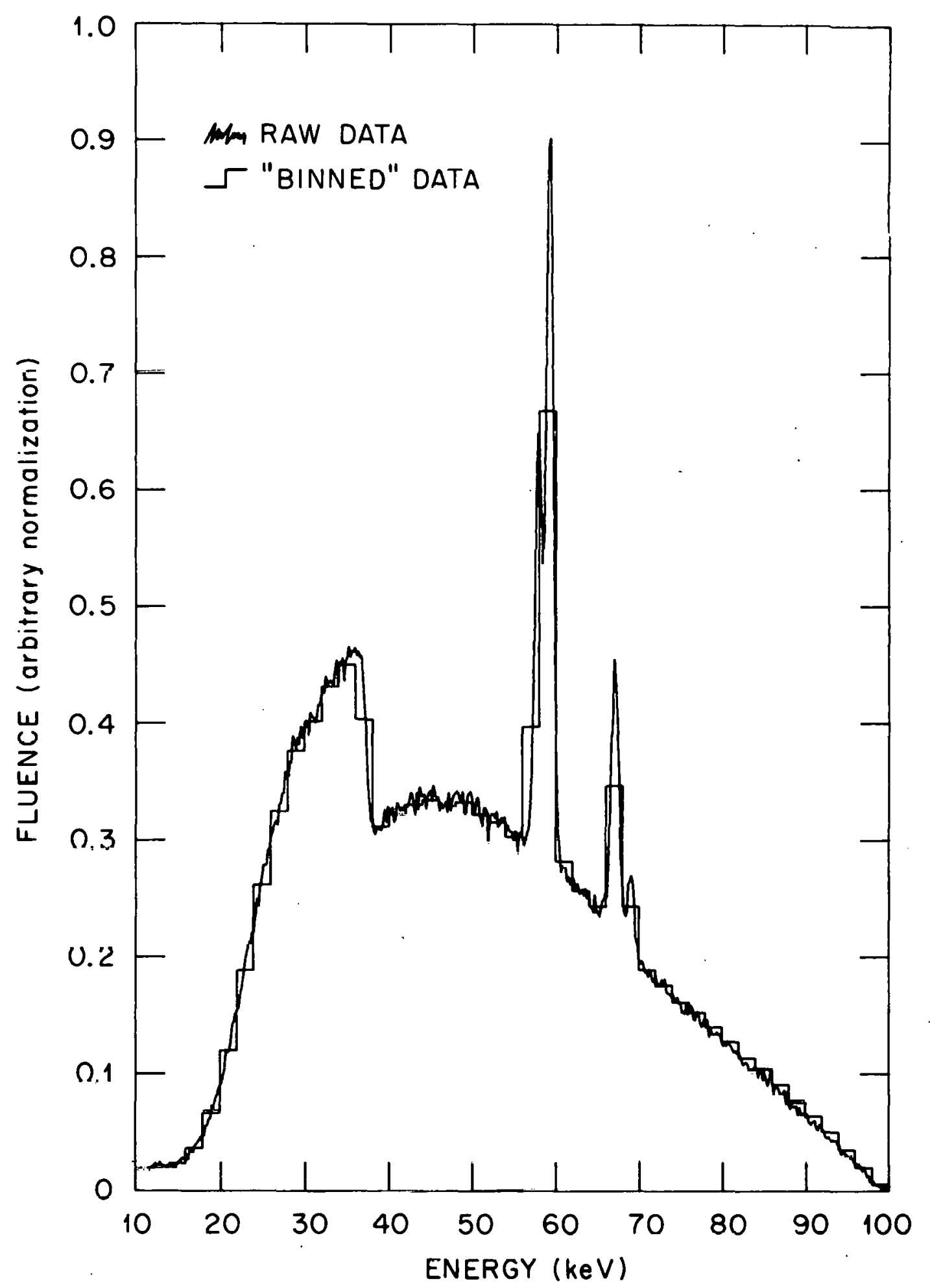

Figure 9. X-Ray Machine Output Spectrum Measured with a Ge(Li) Detector -- $100 \mathrm{kVcp}$. 
pulse height spectra acquired with the $G(L i)$ detector. To facilitate the comparison of these spectra with the spectra acquired with the $\mathrm{NaI}(\mathrm{Tl})$ spectrometer described in the next section, the data were grouped into "bins" $2 \mathrm{keV}$ wide as follows. The raw data were corrected channel by channel to account for intrinsic efficiency losses due to the finite thickness $(7.1 \mathrm{~mm})$ of the $\mathrm{Ge}(\mathrm{L} i)$ detector. Next, the fluence in any "bin" was taken to be the average value of the corrected counts in the raw data channels whose energy lay within the boundaries of the "bin." Both the raw data and the "binned" spectra shown in Figures 7, 8, and 9 have been normalized so that their integrals have the arbitrary value of 1.0 photon/ $\mathrm{cm}^{2}$.

The absorption edge prominent in all three spectra is centered at $37.4 \mathrm{keV}$. This edge was caused by barium contained in the glass envelope of the therapy-type tube, and a similar barium edge has been observed by other researchers studying the spectrum of a similar tube of the same brand (Gi75). An approximate calculation showed that $0.016 \mathrm{gm} / \mathrm{cm}^{2}$ $( \pm 10 \%)$ of barium was necessary to produce an edge the size of the one pictured in Figure 9. If it is assumed that the glass envelope is $0.1 \mathrm{~cm}$ thick where the $x$ rays exit the tube and that the glass comprising the envelope has a density of 3.0 , then the barium content of the glass is approximately $5 \%$ by weight. Barium oxide is one of the materials added to glass to adjust its thermal expansion coefficient., Glass used to make $x$-ray tubes must have a thermal expansion coefficient similar to the metal components which penetrate the glass envelope in order that adequate glass-to-metal high vacuum seals can be made. The amount of barium added to $x$-ray tube glass varies from manufacturer to 
manufacturer and from one batch of glass to another produced by the same manufacturer (Si77).

Figure 10 shows the comparison of the $80 \mathrm{kVcp}$ spectrum measured in this study (Figure 8) with a measured spectrum reported by Atkins as "representative of those used in diagnostic radiology" (At75). This reported $80 \mathrm{kVCp}$ spectrum was produced with a beryllium-windowed x-ray tube with $2 \mathrm{~mm}$ of aluminum added filtration. According to some preliminary and unpublished data furnished by researchers working in the Nationwide Evaluation of X-Ray Trends (NEXT) study, almost all diagnostic $x$-ray tubes have glass windows (Si77). However, according to the results of their unfinished study of spectra from six diagnostic $x$-ray tubes, none of the six tubes contained enough barium to noticeably alter the spectrum. Thus, the spectrum reported by Atkins is probably "more typical" of an $80 \mathrm{kVCp}$ spectrum with $2 \mathrm{~mm}$ aluminum filtration than is the spectrum measured in this study (Figure 8 ). The effect of the difference between the spectral distribution of $x$ rays employed in this study and the spectral distribution of $x$ rays more typical of diagnostic radiology is discussed in the Error Analysis section of Chapter IV of this text.

The focal spot of the $x$ ray tube was determined to be $8 \mathrm{~mm}$ by $8 \mathrm{~mm}$. The determination was made using a $0.5 \mathrm{~mm}$ pinhole collimator and photographic film. The accuracy of the light localizer of the collimator of the $x$-ray machine was checked also with photographic film. The collimator was of the variable therapy type in which a rectangular $x$-ray beam is formed by the two orthogonal pairs of shutter plates. A penumbra was observed around the edge of the $x$-ray beam defined by the $x$-ray 


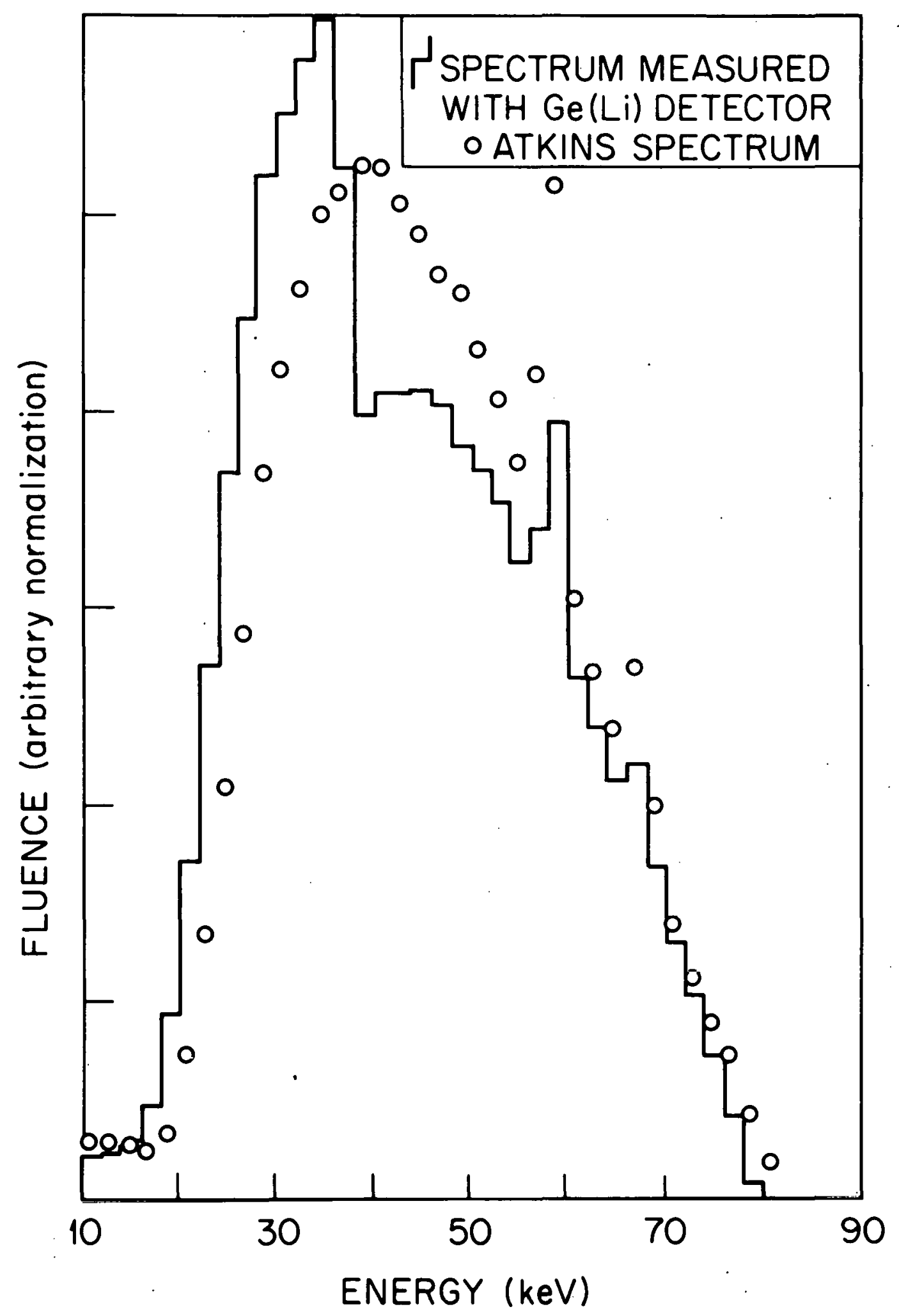

Figure 10. Comparison of X-Ray Machine Output Spectrum with a Representative Diagnostic X-Ray Spectrum. 
machine collimator. At a focal-spot-to-film distance of $1 \mathrm{~m}$ the penumbra was $0.75 \mathrm{~cm}$ wide. A typical diagnostic $x$-ray machine, having a smaller focal spot (1 or $2 \mathrm{~mm}$ square), would have a correspondingly smaller penumbra.

In summary the $x$-ray machine met or exceeded all of the design criteria except that concerning the simulation of diagnostic $x$-rays. The shape of the spectrum of radiation produced by the $x$-ray machine only approximates the spectral shape typical of a diagnostic $x$-ray machine. Also the large focal spot and accompanying penumbra are several times larger than those typical of diagnostic $x$-ray machincs. In order to be a better simulation, a tube with low barium content in the glass and with a smaller focal spot would be needed. Nevertheless since a better $x$-ray tube was not readily available, the above $x$-ray machine was used to complete the study. It was felt that the size of the penumbra would not affect the results of this study drastically. On the other hand because the spectral shape of the radiation produced by the $x$-ray machine does not simulate closely diagnostic $x$-ray spectra, care should be used in interpreting and applying the data presented in this study.

\section{Spectrometer .}

A spectrometer that was compact, had a uniform response over all solid angle, and was capable of being used in a fluid-filled anthropomorphic phantom was desired. A NaI(Tl) scintillation spectrometer meeting or coming close to these criteria was designed and is shown in Figure 11. The scintillation crystal was to be a sphere of $6.3 \mathrm{~mm}$ diameter which was half-imbedded in a polyvinyltoluene light pipe. 


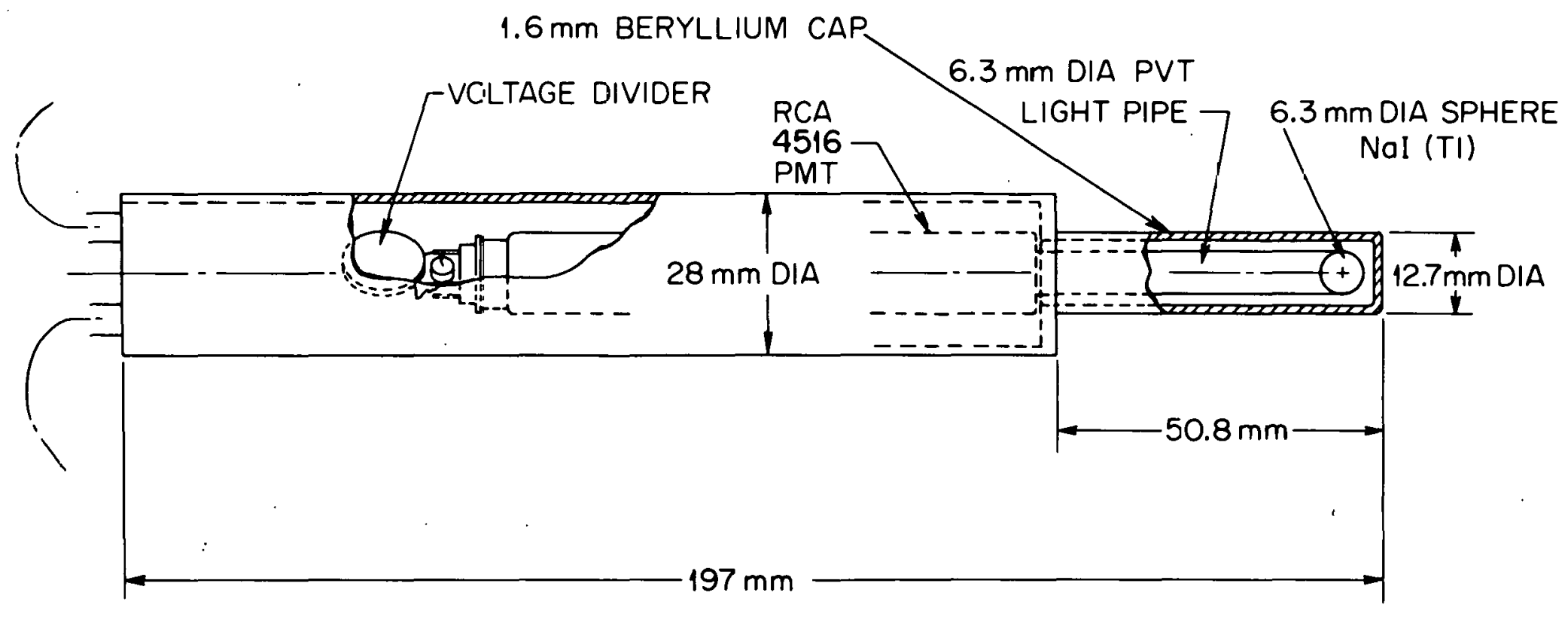

Figure 11. NaI(Tl) Scintillation Detector. 
The light pipe was to be optically coupled to an RCA 4516 photomultiplier tube. The scintillator and light pipe were to be covered by a $1.6 \mathrm{~mm}$ thick beryllium cap. The phototube and its ancillary voltage divider were to be covered with a magnetic shield, and the entire probe was to be hermetically sealed.

After some difficulty and delay, two probes resembling this design were procured. A major deviation from design specifications was that the detectors accepted have scintillation crystals that were slightly oblate. As determined by radiographs, the diameter of each crystal, parallel to the axis of the light pipe was $5.7 \pm 0.1$ imin and the diameter perpendicular to the axis of the light pipe was $6.2 \pm 0.1 \mathrm{~mm}$.

One of the probes had a polished $\mathrm{NaI}$ crystal, and both the crystal and light pipe were covered with a specularly reflecting, thin aluminum foil. The other probe had a crystal with a roughened surface, and, in this case, both the crystal and light pipe were covered with a diffuse reflector (white paper). The detector with the unpolished crystal and diffuse reflector exhibited a slightly better resolution and was selected for use in this study.

A third probe was procured also. This probe was similar to the others except that the NaI crystal was not included. This probe was used in a preliminary set of measurements to determine the significance of scintillations in the polyvinyl toluene light pipe and $x$-ray interactions in the photocathode and first dynode. The investigations revealed that such interactions were insignificant. In fact, no counts above the lower level discriminator were observed when the blank probe was exposed in situations identical to those in which the regular probe was used to acquire data. 
The magnetic shielding for the probe used in this study was found to be inadequate early in testing of the detector. Orientation of the probe with respect to the earth's magnetic field influenced the gain of the photomultiplier tube by as much as $\pm 10 \%$. This problem was alleviated by wrapping the photomultiplier tube housing with several layers of "mu-metal," an alloy of high magnetic permeability.

Two sets of measurements were performed to determine the uniformity of response of the detector with respect to orientation of the beam of impinging photons. Three sources were used: ${ }^{57}$ Co (122 keV gamma ray), ${ }^{241} \mathrm{Am}$ (60 keV gamma), and ${ }^{109} \mathrm{Cd}$ (Ag x-rays with average energy of $22 \mathrm{keV})$. The orientation of the beam of photons was defined by a celestial coordinate system with the plane of the equator passing through the center of the spherical crystal perpendicular to axis of the light pipe. In the first set of measurements data were acquired for sources positioned at various azimuths in the plane of the equator. For each source no significant change in pulse height distribution was observed.

In the second set of measurements, the sources were positioned at locations having varying declinations but a constant azimuth. The mean of the pulse height distribution varied as a function of declination and energy as shown in Figure 12. This variation is believed to be a result of changing light collection efficiency. It should be noted that the mean of the pulse height distribution varies less than $10 \%$ over the large solid angle contained between declinations of $\pm 45^{\circ}$. This solid angle is $70 \%$ of $4 \pi$ steradians. Obviously, any photons passing through the photomultiplier tube housing would have a high probability of being absorbed there. However, since the housing subtends a solid angle of 


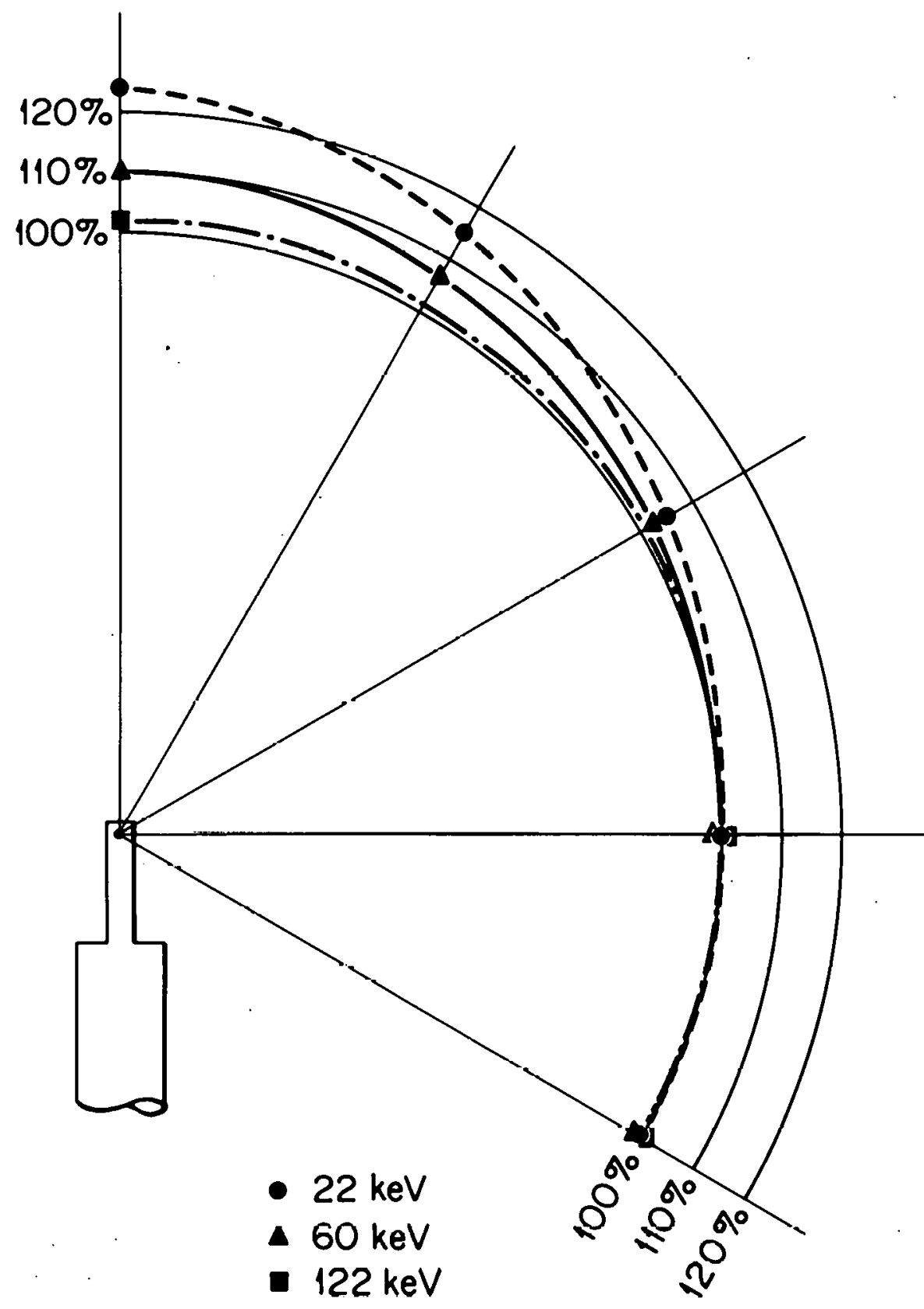

Figure 12. Change in Mean of Pulse Height Distribution as a Function of Declination and Energy of Incident Beam (Data are Normalized so that Side Response Equals 100\%). 
less than $2.5 \%$ of the total solid angle viewed by the NaI crystal, effects of this nonuniformity can be minimized by careful positioning of the probe within an ambient $x$-ray field.

Next the resolution and linearity of the detector were determined as a function of incident energy. Measurements were made for side incidence with photons from isotopic sources and with monochromatic $x-$ rays. The mean and standard deviation of a pulse height distribution were determined by using a computer-coded least squarès algorithm to fit a Gaussian to the multichannel analyzer data.

Sources of ${ }^{57} \mathrm{Co}$ and ${ }^{241} \mathrm{Am}$ were used as before and a ${ }^{109} \mathrm{Cd}$ source, shielded with copper foil, was used to produce $88 \mathrm{keV}$ photons. Monoenergetic sources from $15 \mathrm{keV}$ to $50 \mathrm{keV}$ were produced using a bent crystal $\mathrm{NaCl} x$-ray monochromator. The $\mathrm{NaCl}$ crystal used had a rocking angle of $0.1^{\circ}$.

Since an $0.1^{\circ}$ collimator slit was used to enhance the production of a highly monochromatic beam, the line source output of the monochromator was not large enough to expose the entire active volume of the detector. Therefore, the response of the detector was determined in the following manner. Seven zones of a sphere were chosen so that each

zone had an equal projected area. The detector was exposed to the monochromatic source and the mean and standard deviation were determined at seven locations along the long axis of the detector. The seven locations were centroids of the projected areas of the seven zones. The mean of the pulse height distribution for a uniform side irradiation was taken as the average of the means measured at the seven locations. The corresponding standard deviation was computed after noting that the 
variance of a sum of distributions is equal to the variance of the means plus the mean of the variances of the distributions comprising the sum. The results of linearity and resolution measurements are presented graphically in Figures 13 and 14. The linearity data was analyzed in two regions, the region above $32 \mathrm{keV}$ and the region below. The discontinuity at $32 \mathrm{keV}$ is believed to be due to a variation in light collection efficiency for scintillations resulting from $x$-rays with energy near the K-edge of iodine. The $122 \mathrm{keV}$ point from the ${ }^{57} \mathrm{Co}$ source and the $35 \mathrm{keV}$ point from the $x$-ray monochromator were judged to be the most accurate and were used to determine the line representing the linearity in the upper region. The 30 and $20 \mathrm{keV}$ points were used to determine the linearity in the lower region. The line in the resolution graph (Figure 14) was determined by a "visual best fit." Again the ${ }^{57}$ Co point was judged highly reliable. Note that the resolution of this detector is not as good as the resolution of a more conventional $\mathrm{NaI}$ spectrometer. The resolution of this detector, expressed as the ratio of full-width at half-maximum (FWHM) to the mean, was found to be $52 \%$ at $122 \mathrm{keV}$. The corresponding resolution (FWHM) for a $7.6 \mathrm{~cm} \times 7.6 \mathrm{~cm}$ (dia.) $\mathrm{NaI}(\mathrm{Tl})$ spectrometer would be about $12 \%$ (Is71).

The equipment ancillary to the $\mathrm{NaI}\left(\mathrm{T}_{\ell}\right)$ spectrometer included a preamplifier, a linear amplifier and a multichannel analyzer. The preamplifier was designed by Thorngate (Th76). The multichannel analyzer had 1024 channels and a $100 \mathrm{MHz}$ digitizing rate. To facilitate data handling, the analyzer was interfaced with a teletype equipped with a paper tape punch. 


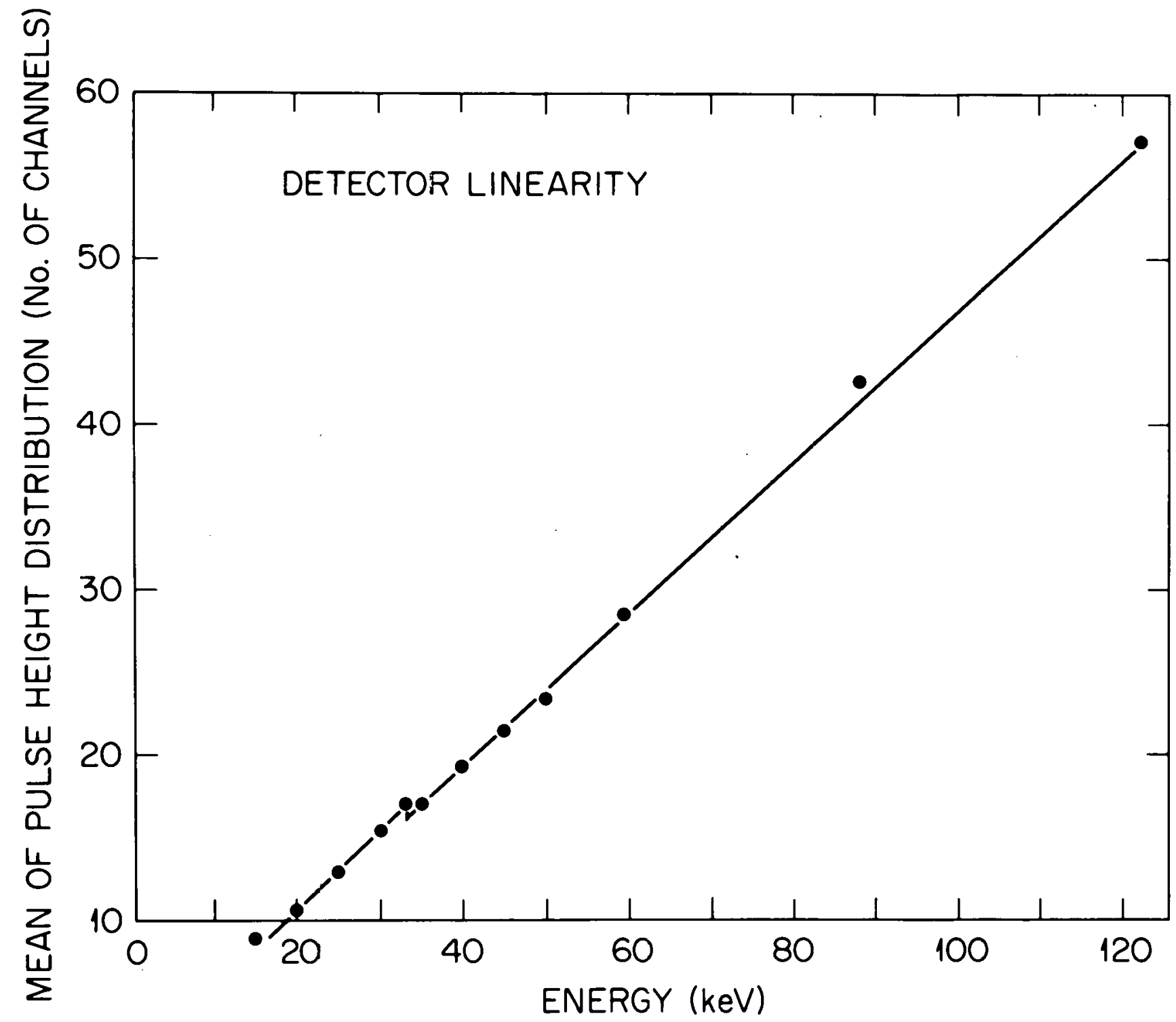

Figure 13. Mean of Pulse Height Distribution versus Energy for the Spherical $\mathrm{NaI}(\mathrm{Tl})$ Detector. 


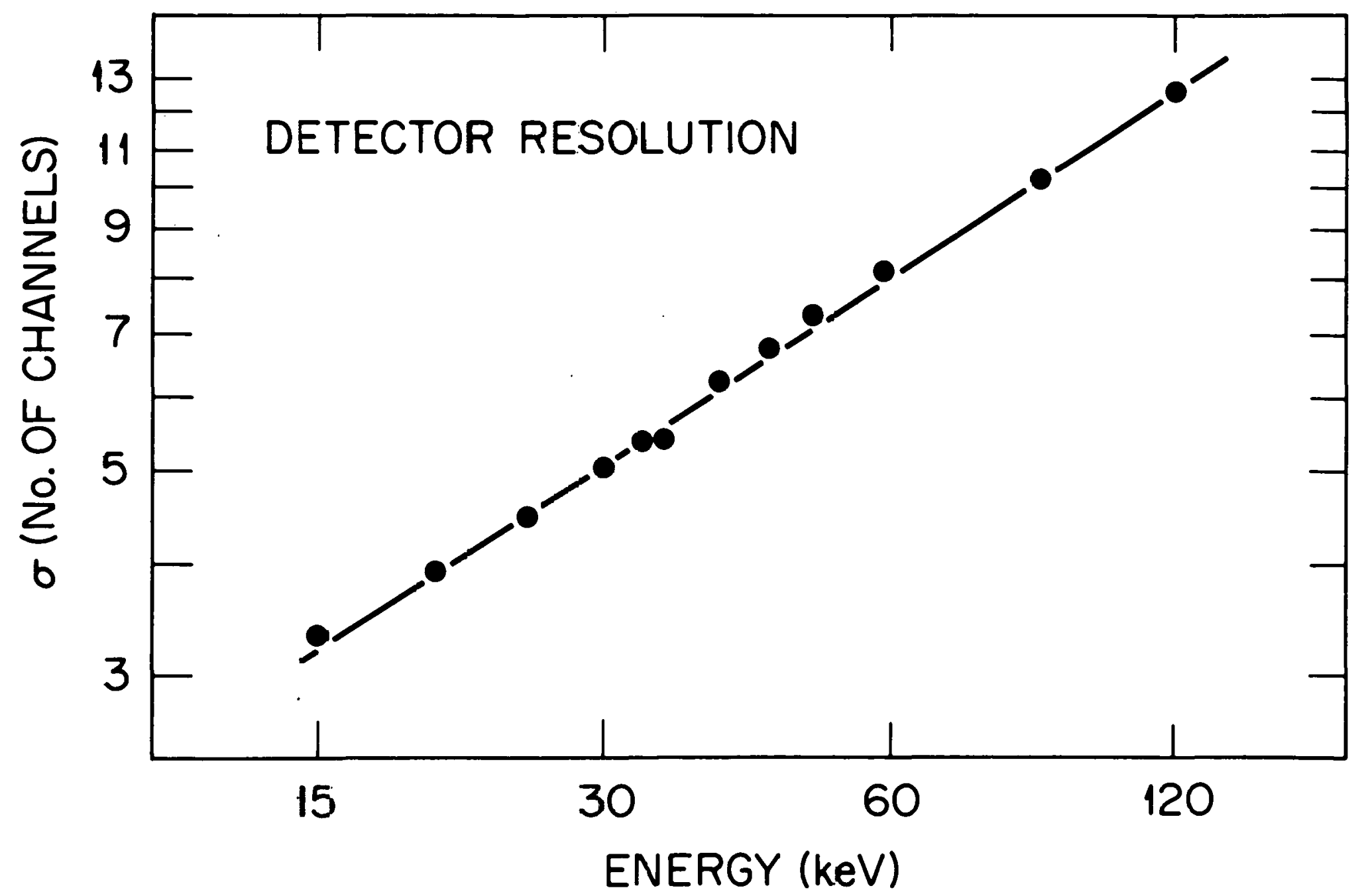

Figure 17. Sigma of Puise Height Distributicn versus Energy for the Spherizal NaI!TE) Detector. 


\section{Unfolding Procedure}

The unfolding scheme outlined by Equations 2-16 and 2-17 requires a complete set of response functions $\left\{R_{E_{j}}\left(N_{k}\right)\right\}$ where $E_{j}$ is the energy of the uniformly incident monoenergetic beam and the argument $N_{k}$ denotes that each function is a distribution over channel number. To obtain a set of response functions, the first step was the computation of the set of deposited energy distributions $\left\{R^{\prime} E_{j}\left(E_{k}\right)\right\}$ where $E_{j}$ again indicates the incident energy and the argument $E_{k}$ denotes that each function is a distribution over energy. A photon entering the detector may (1) escape, (2) be photoelectrically absorbed, or (3) be Compton or coherently scattered. Since coherent scattering does not result in any appreciable energy absorption it was not considered in this treatment.

After a photon is photoelectrically absorbed, it may give rise to a fluorescent $x$ ray. The fluorescence yield of sodium is small (3\%). In addition, the low energy sodium fluorescence $x$-rays would have a very short mean free path in sodium iodide. Therefore, sodium fluorescence also was not considered in this treatment. However, the fluorescent yield of iodine is quite high $(86 \%)$ and the $K_{\alpha}$ and $K_{\beta}$ groups of $x$-rays have average energies of 28.5 and $32.5 \mathrm{keV}$, respectively. Since the emission of a fluorescent $x$ ray is isotropic, the probability averaged over all solid angle of a fluorescent $x$ ray produced at a location $\vec{r}$ escaping from a spherical crystal is

$$
P(r)=\frac{1}{2 \mu r} e^{-\mu r_{0}} \sinh (\mu r)+\mu\left(r_{0}^{2}-r^{2}\right) \int_{\mu\left(r_{0}-r\right)}^{\mu\left(r_{0}+r\right)} \frac{e^{-x}}{x^{2}} d x
$$

where $\mu$ is the linear attenuation coefficient, $r_{0}$ is the radius of the 
sphere, $\vec{r}$ is the vector which locates the point of fluorescent $x$-ray production with respect to the center of the sphere, $r$ is the magnitude of $\vec{r}$, and sinh is the hyperbolic sin function.

Photons which are Compton scattered within the spherical crystal cannot be treated so simply. Although the energy-angle relationship for Compton scattering is not too complex (see Equation 2-5), the scattering is not isotropic. The probabllity for scatter in a given direction with respect to the direction of the photon before scattering is given by the Klein-Nishina relation (Ev65). The Klein-Nishina relation is exact but does not lend itself to integration in a complex calculation. Also, since Compton-scattered photons may be multiply scattered before escaping or being absorbed, it was decided to calculate the deposited energy distributions with Monte Carlo techniques.

The distributions of energy deposited in a spherical crystal of $\mathrm{NaI}$ for incident monoenergetic beams of $10,12,14, \ldots, 130 \mathrm{keV}$ were calculated. The results of the calculations for each incident energy were grouped into energy "bins" which were $2 \mathrm{keV}$ wide. Figure 15 shows the results of the calculations for one of the 61 incident energies. The peak at $110 \mathrm{keV}$ indicates the "full energy peak." The peaks at 78 and $82 \mathrm{keV}$ indicate events in which a $K_{\alpha}$ or $K_{\beta}$ iodine $x$ ray escaped the $\mathrm{NaI}$ crystal. The distribution below $36 \mathrm{keV}$ indicates events in which a Compton scattered photon escaped the detector. Next the $\left\{R^{\prime} E_{j}\left(E_{k}\right)\right\}$ were converted to $\left\{R_{E_{j}}\left(N_{k}\right)\right\}$ by using the resolution and linearity data acquired as described in the previous section of this chapter. Figure 16 shows the response function for the same incident energy used in Figure 15. 


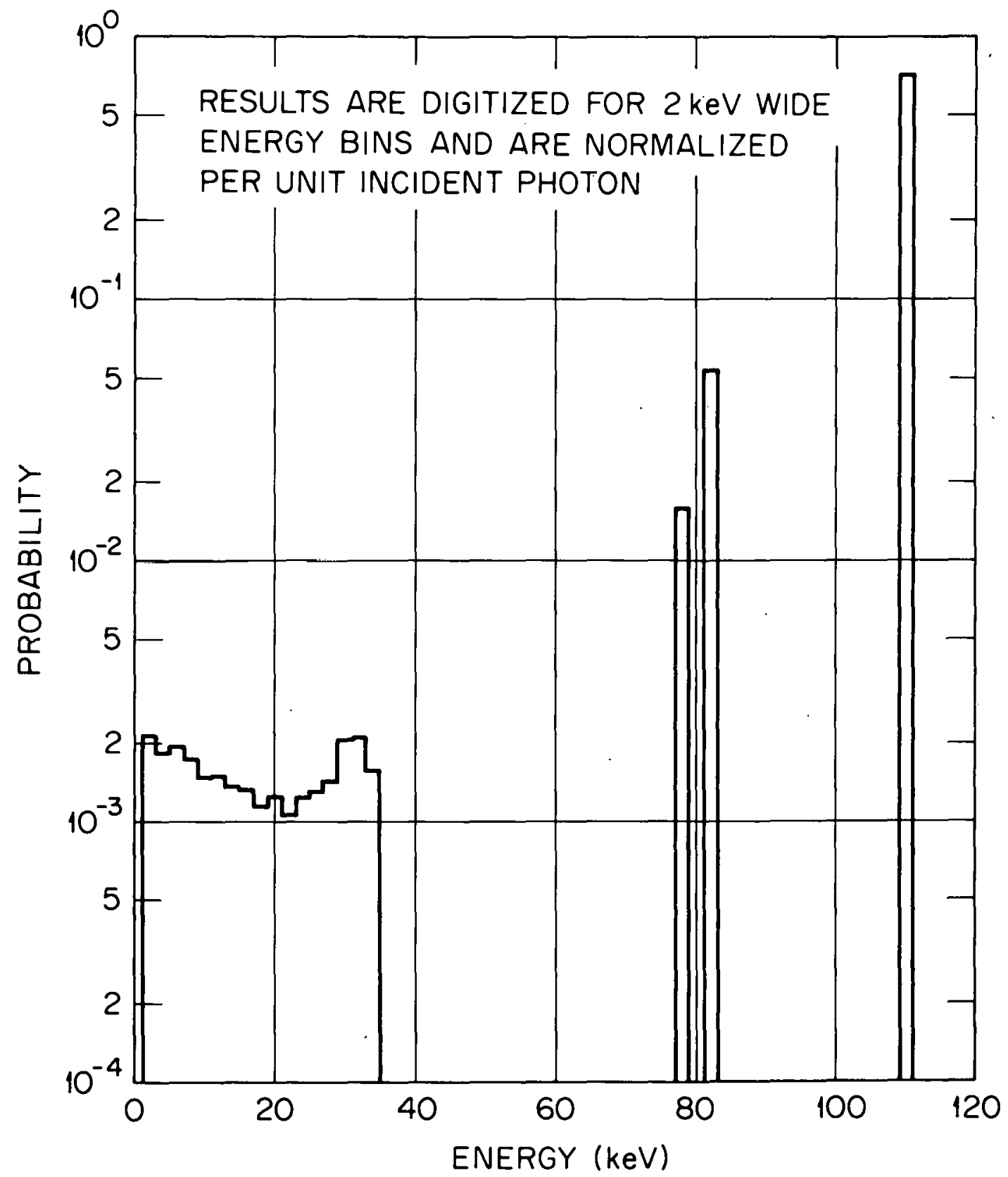

Figure 15. Calculated Distribution of the Energy Deposited in the NaI Crystal of the Detector by a Uniform Parallel Beam of $110 \mathrm{keV}$ Photons. 


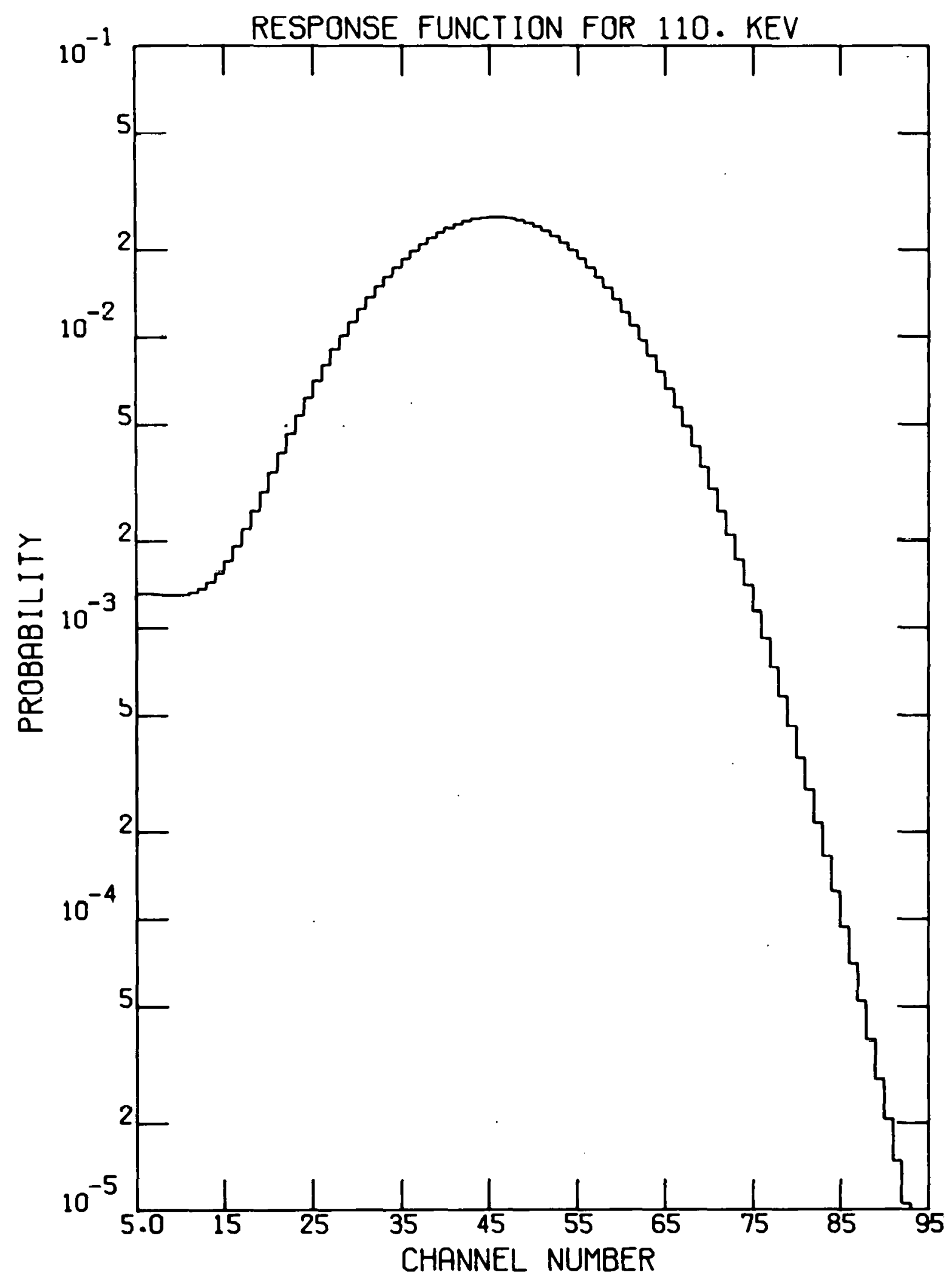

Figure 16. Calculated Response Function for $110 \mathrm{keV}$ Uniformly Incident Photons. 
The fluence, $\Phi(E)$, was computed from the observed pulse height spectrum, $R(N)$, with a computer-coded, iterative technique. The technique has three basic steps:

1. A trial pulse height spectrum $R_{j}\left(N_{k}\right)$ was calculated with

$$
R_{j}\left(N_{k}\right)=\sum_{j} \Phi_{i-l}\left(E_{j}\right) \times R_{E_{j}}\left(N_{k}\right)
$$

where the subscript $\underline{i}$ indicates the iteration number, $\Phi_{i-1}$ is the estimate of the fluence resulting from the previous iteration, the subscript $\underline{j}$ indicates summation over all non-zero elements of $\Phi_{i-1}\left(E_{j}\right)$, and the subscript $\underline{k}$ indicates that the computation is made for each channel number. For the first iteration $\Phi_{i-1}\left(E_{j}\right)$ is set equal to one for all $E_{j}$.

2. A trial fluence $\Phi_{i}\left(E_{j}\right)$ is calculated from the previous trial fluence $\Phi_{i-1}\left(E_{j}\right)$ with

$$
\Phi_{i}\left(E_{j}\right)=\Phi_{i-1}\left(E_{j}\right) \times \sum_{k}\left(\frac{R\left(N_{k}\right)^{q}}{R_{j}\left(N_{k}\right)^{q}} \times R_{E_{j}}^{\star p}\left(N_{k}\right)\right)
$$

where the subscript $\underline{k}$ indicates that the summation is computed for all non-zero elements of $R_{j}\left(N_{k}\right)$ and the subscript $\underline{j}$ indicates that the computation is made for each element in $\Phi_{i-1}\left(E_{j}\right)$. The asterisk in $R_{E_{j}^{\star}}^{P}\left(N_{k}\right)$ indicates that this distribution is normalized over $k$, that is 


$$
R_{E_{j}}^{\star p}\left(N_{k}\right)=\frac{R_{E_{j}}\left(N_{k}\right) p}{\sum_{k} R_{E_{j}}\left(N_{k}\right)^{p}}
$$

The superscripts $p$ and $q$ have the value of either 1 or 2. Setting either or both equal to 2 increases the speed with which the iteration process converges.

3. The next step is one in which the results of the iteration are evaluated. The residuals, S, are computed by

$$
S=\frac{\sum_{k}\left(R_{i}\left(N_{k}\right)-R\left(N_{k}\right)\right)^{2}}{\sum_{k} R\left(N_{k}\right)^{2}}
$$

Thus $S$ is a measure of how well the trial pulse height spectrum approximates the measured pulse height spectrum. In fact, the square root of $S$ is the rootmean-square of the difference between the trial and observed pulse height spectrum expressed as a fraction of the average value of the observed pulse height spectrum. If $S$ is less than some pre-determined value, the iteration process is ended, and the final $\Phi_{j}\left(E_{j}\right)$ is taken to be an estimate of $\Phi(F)$, the fluence which actually caused the observed pulse height spectrum. This unfolding procedure is similar to one used to analyze electron swarm data (Mc72). 
In this study $S$ was chosen to be less than 0.0003 . Hence the fractional-root-mean-square difference between the trial and observed pulse height spectra was less than $1.8 \%$. Examination of data taken previously showed that for a given exposure situation (i.e., x-ray beam site and location, detector position and orientation, generating potential, tube current, etc.), slightly different pulse height spectra would be observed. Statistical tests showed that the variation in a number of counts in a given channel was Poisson in nature. The standard deviation of the number of counts in a given channel, taken from a number of pulse height spectra acquired under similar conditions, was approximately equal to the square root of the mean of the number of counts. Most of the pulse height spectra which were unfolded in the course of this research had a maximum number of counts per channel between 50,000 and 100,000. Since these spectra approached zero on each end, there were a number of channels for which the expected standard deviation was on the order of $1 \%$ or greater. Thus requiring the residuals, $S$, to be smaller than 0.0003 would be requiring that the fractional root-mean-square difference between the trial and observed pulse height spectra be smaller than the expected error for a large number of channels in the observed spectrum. Nevertheless the selection of 0.0003 as the maximum allowable value of the residuals is somewhat arbitrary.

The spectral output of the $x$-ray machine was measured with the spherical NaI detector and with a $\mathrm{Ge}(\mathrm{Li})$ spectrometer. The Ge(Li) spectra were corrected for efficiency losses and were grouped into energy "bins", $2 \mathrm{keV}$ wide. The NaI spectra were unfolded with the procedure defined above. The raw pulse height distributions, the unfolded NaI 
spectra, and the corresponding $\mathrm{Ge}(\mathrm{L} i)$ spectra for generating potentials of 60 and $80 \mathrm{kVCp}$ are presented in Figures 17 and 18. The spectra are normalized per unit fluence. Also the absorbed dose in reference man soft tissue was calculated for each unfolded spectrum with

$$
D=c \sum_{k} \frac{{ }_{\text {en }}}{\rho} E_{k} \Phi\left(E_{k}\right)
$$

where $\mu_{\mathrm{en}} / \rho$ is the mass energy absorption coefficient and $c$ is the factor necessary to convert keV per gram to units of absorbed dose.

The correspondence of the unfolded $\mathrm{NaI}$ spectra and the spectra measured with the $\mathrm{Ge}(\mathrm{Li})$ detector was taken as the best evidence that the unfolding procedures resulted in a measured spectrum which was a realistic estimate of the actual spectrum incident upon the spherical NaI detector. It should be noted that the unfolded $\mathrm{NaI}$ spectrum does not accurately represent the incident spectrum in a neighborhood around the barium $\mathrm{K}$-edge and around the upper energy limit of the incident spectrum. According to Anderson this "rounding of the corners" was a natural consequence of the poor resolution of the NaI spectrometer (An76).

The inability of the spectrometer to resolve discontinuities similar to the barium edge is judged unimportant becausc it is felt that $x$-ray spectra present inside a phantom would not have such discontinuities. There are only trace quantities of elements present in the human body (or in phantoms to represent the body) which have K-edges above $10 \mathrm{keV}$. Also any discontinuities in a spectrum incident on a phantom would be smoothed to some degree by the scattering processes within the phantom. 


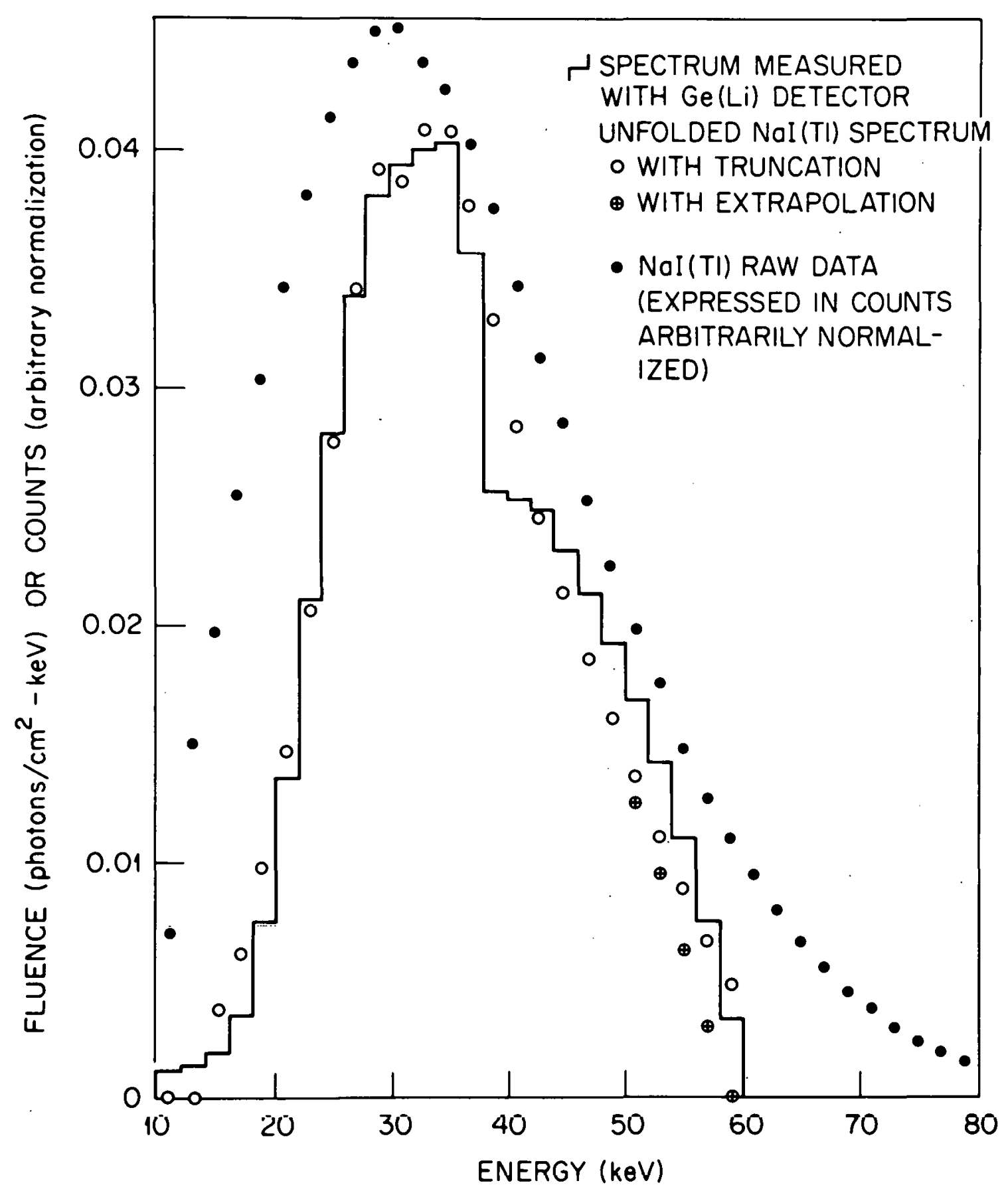

Figure 17. Comparison of Unfolded NaI(Tl) Spectrum with Spectrum Measured with a $\mathrm{Ge}(\mathrm{L} 1)$ Detector -- $60 \mathrm{kV} \mathrm{cp}$. 


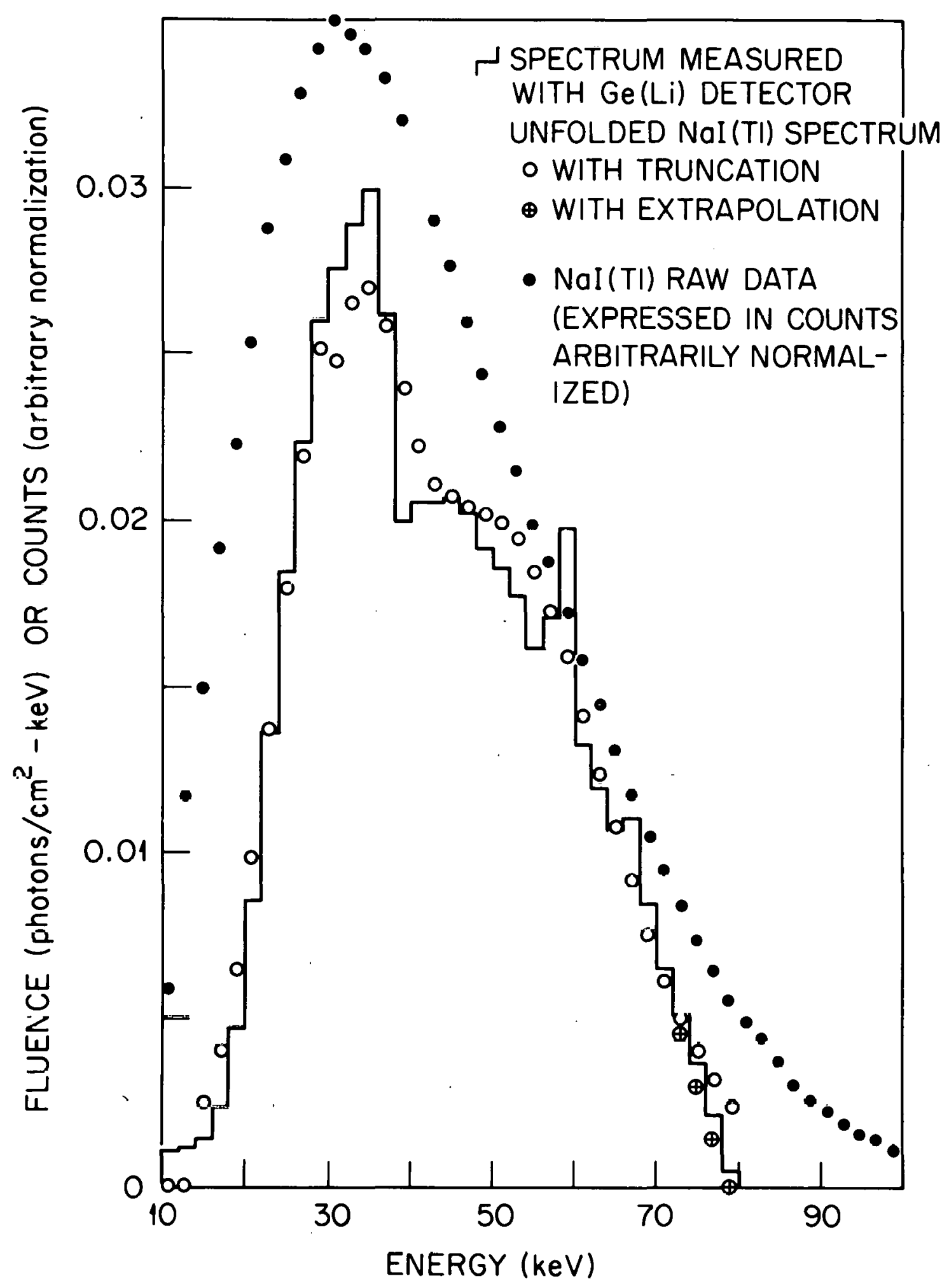

Figure 18. Comparison of Unfolded $\mathrm{NaI}(\mathrm{Tl})$ Spectrum with Spectrum Measured with a Ge(Li) Detector -- $80 \mathrm{kVCp}$. 
The inability of the unfolding processes to produce a spectrum which accurately represents the spectrum incident on the detector near the upper energy limit was judged to be a serious drawback to the use of this $\mathrm{NaI}$ detector. This drawback was dealt with in two ways in treating the data presented in subsequent chapters. First a numerical technique was used to compute the first derivative of a second order polynomial fit via least squares to a point in the unfolded spectra and its two nearest neighbors on both sides (5 point fit) (Sa64). If this first derivative, computed for several points just below the energy of the generating potential, displays a relative minimum (i.e., largest negative slope) then the unfolded spectrum was adjusted. The adjustment was accomplished by replacing the data with energy greater than the point of minimum first derivative with the value obtained by linearly extrapolating from the point of minimum derivative with slope equal to that derivative. All negative values were truncated to zero. If the unaltered, unfolded spectrum failed to have a minimum of its first derivative in the neighborhood just below the generating potential, the unfolded spectrum was truncated to zero above the generating potential. Figure 19 shows the effect of extrapolation and truncation on a spectrum produced at $80 \mathrm{kVcp}$.

Figure 19 presents two unfolded spectra along with the absorbed doses for each spectrum calculated with Equation (3-6). and the centroids for each spectrum (denoted by the short vertical tick mark). Both spectra were unfolded from pulse height spectra collected with a generating potential of $80 \mathrm{kVcp}$. Extrapolation reduced the calculated centroid by 


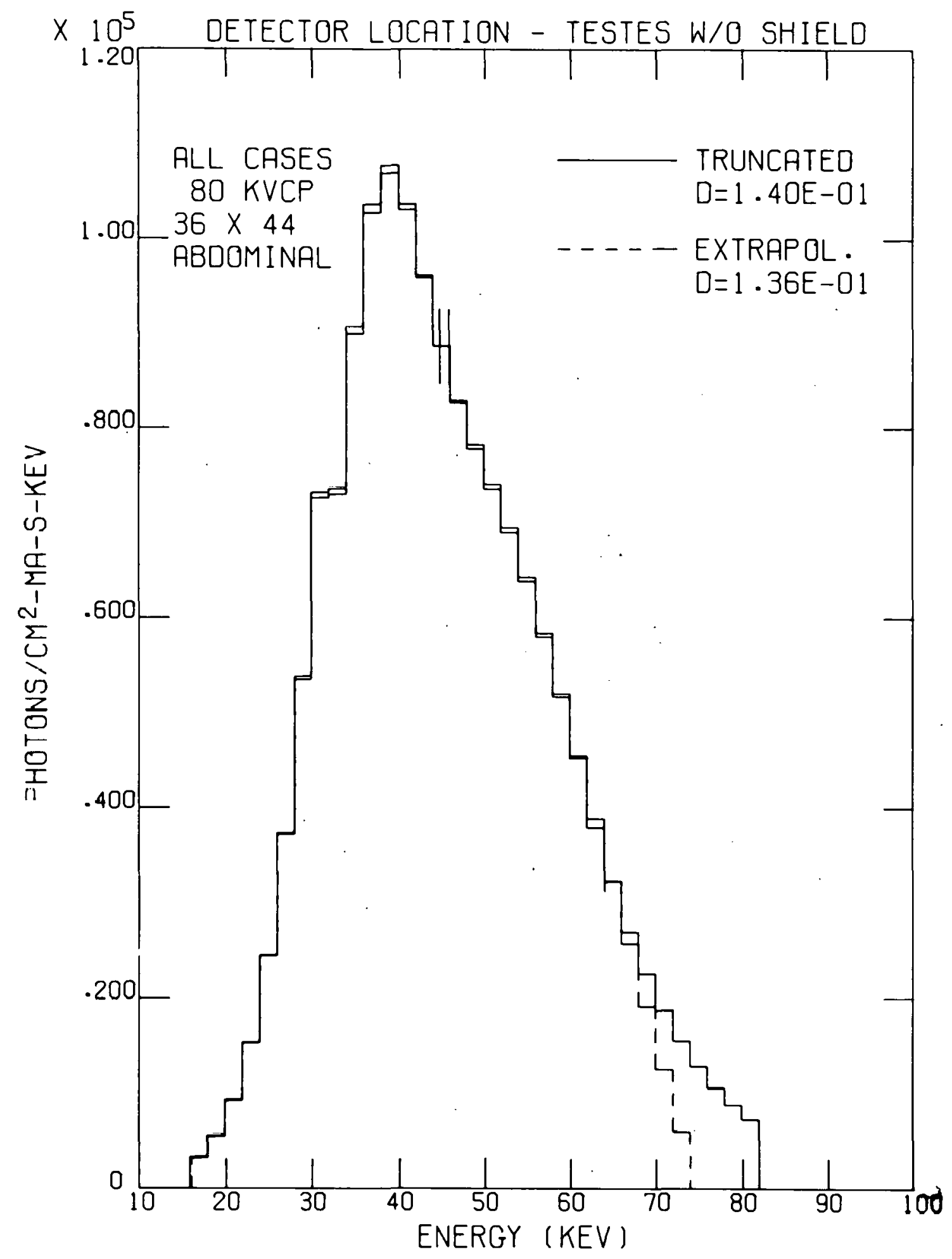

Figure 19. An Example of Truncation and Extrapolation. Vertical ticks indicate mean energy. Field size is in $\mathrm{cm}$. D is the absorbed dose per unit current per unit time (mrad/mA-s). 
approximately $1 \mathrm{keV}$ with respect to the centroid calculated for the truncated spectrum. Also extrapolation reduced the calculated absorbed dose by $3 \%$ with respect to the absorbed dose calculated for the truncated spectrum.

The truncation of the unfolded spectra above the generating potential was justified on the basis that no photons produced by an $x$-ray machine can have an energy greater than the kinetic energy of the electrons bombarding the $x$-ray tube target. The extrapolation procedure was based on the assumption that as a bremsstrahlung spectrum almost linearly approaches zero near its upper energy limit, so must a scattered bremsstrahlung spectrum.

\section{MR. ADAM Phantom}

In 1976, the ICRP published its report called Reference Man. This report contains a wide range of anatomical data for a model representative of an "average" adult human (ICRP75). Many of the data for this ICRP report were compiled over a period of years by the staff of the Medical Physics and Internal Dosimetry Section of the Health Physics Division of the Oak Ridge National Laboratory. While these data were being acquired, a model based on the same data was developed by the same section for use in Monte Carlo calculations (Fi67). In the model the three major body regions (head, trunk, and legs) and 23 major organs of interest were described by segments of general quadratic surfaces such as spheres, ellipsoids, elliptical cylinders, and flat plates. This calculational model was used to estimate the distribution of absorbed dose among the various organs and regions for sources of ionizing radiation both inside and outside 
the model (Jo76, Po73, Po74a, Po74b, Sn73, Sn74). In 1969 the Medical Internal Radiation Dose Committee of the Society of Nuclear Medicine adapted this model for use in estimating the absorbed dose to patients administered radionuclides internally for medical purposes (Sn69). In 1974 the model was modified slightly to make it more suitable for calculations of radiation dose distributions from external sources of radiation. The revised model was used in calculations of dose distributions to assist the U.S. Bureau of Radiological Health in assessing medical diagnostic $x$-ray exposures (Po74b, Ro76a, Ro76b).

In 1974 a phantom which was a physical mock-up of the mathematical model was built. Subsequently the phantom was used in several programs of measurements to provide experimental data for intercomparison with calculated data (Ga74, Me75, Sta75). When the mathematical model was modified, the phantom was modified also to reflect these changes (Me75).

The MR. ADAM phantom (Mock-up of a Representative Analytical Description of an Adult Male), built for this study, is pictured in Figure 20. The phantom and the mathematical model which it represents have three body regions, head, trunk, and legs. Both phantom and model are comprised of subregions of different materials: skeletal, lung, and the rest (called "soft tissue"). The mathematical model, referred to as the Snyder-Fisher phantom, specified the nature of each subregion by giving the chemical composition and density. These specifications are the ones upon which "reference man" is based. Note that the skeletal region is considered to be an homogeneous mixture of hard bone, bone marrow, connective tissue, and the amount of blood normally resident in the bones. Likewise the other regions have an average composition and are completely homogeneous. 


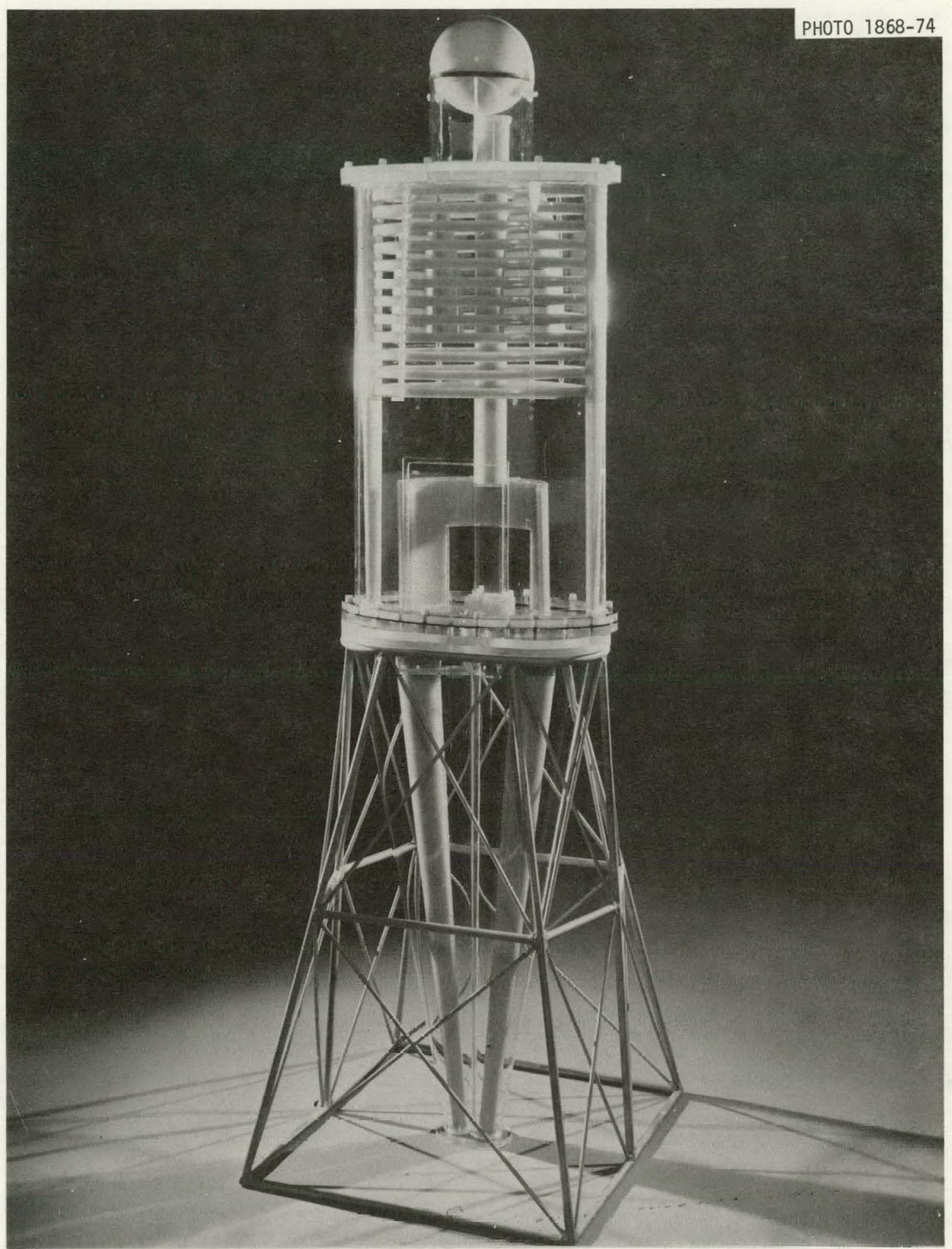

Figure 20. The MR. ADAM Phantom. 
The shells containing the body regions and skeletal and lung subregions of the MR. ADAM phantom were constructed of lucite or polystyrene. The materials used to represent the elemental compositions specified in the Snyder-Fisher phantom were formulated using a computer program. Also the equivalence of the materials with respect to what they represent was assessed using the same program (St74). The criteria used for assessing the equivalence of the materials were to compare the partial mass attenuation coefficients and the density. Table 2 gives the elemental composition and density of the Snyder-Fisher phantom and the comporition, chemical composition, and density of the substitute materials. Tables 3-5 give the partial mass-attenuation coefficients of the substitute materials and their comparisons with the coefficients calculated for Snyder-Fisher phantom specifications. Note that in most cases the coefficients agree within $\pm 1 \%$.

The MR. ADAM phantom was chosen for use in this study for three reasons. First, it resembles the Snyder-Fisher phantom, an accepted phantom of standard geometry. Second, since the phantom is mostly fluidfilled, it allowed access to much of the interior with a probe the size of the spherical $\mathrm{NaI}$ spectrometer. Third, experimentally-determined data medsured in the MR. ADAM phantom have the potential for being directly compared with corresponding data calculated in the Snyder-Fisher phantom. 
Table 2. Description of Snyder-Fisher phantom subregions and their MR. ADAM phantom equivalents

\begin{tabular}{|c|c|c|c|c|c|c|}
\hline & \multicolumn{2}{|c|}{ Soft tissue } & \multicolumn{2}{|c|}{ Skeleton } & \multicolumn{2}{|c|}{ Lung } \\
\hline & Snyder-Fisher $^{a}$ & Equivalent $^{b}$ & Snyder-Fisher ${ }^{a}$ & Equivalent ${ }^{c}$ & Snyder-Fisher ${ }^{a}$ & Equivalent $^{a}$ \\
\hline \multicolumn{7}{|c|}{ Elemental composition, $\%$} \\
\hline $\mathbf{H}$ & 10.474 & 11.26 & 7.036 & 6.47 & 10.208 & 9.78 \\
\hline C & 23.020 & 18.36 & 22.793 & 19.15 & 10.008 & 11.11 \\
\hline $\mathbf{N}$ & 2.339 & & 3.865 & 3.94 & 2.802 & \\
\hline 0 & 63.2106 & 69.38 & 48.559 & 52.98 & 75.958 & 77.61 \\
\hline $\mathrm{Na}$ & 0.128 & 0.393 & 0.315 & 0.17 & 0.190 & 0.59 \\
\hline $\mathbf{M g}$ & 0.016 & & 0.111 & 0.18 & 0.008 & \\
\hline $\mathbf{P}$ & 0.236 & & 6.937 & 6.80 & 0.081 & \\
\hline s & 0.221 & & 0.169 & 0.01 & 0.230 & \\
\hline $\mathrm{Cl}$ & 0.141 & 0.607 & 0.139 & & 0.270 & 0.91 \\
\hline $\mathbf{K}$ & 0.208 & & 0.145 & & 0.200 & \\
\hline $\mathrm{Ca}$ & & & 9.914 & 10.30 & 0.007 & \\
\hline $\mathrm{Fe}$ & 0.006 & & 0.008 & & 0.037 & \\
\hline $\mathrm{Zn}$ & 0.005 & & 0.010 & & 0.002 & \\
\hline Density, $\mathrm{g} / \mathrm{cm}^{3}$ & 1.0 & $0.99 \pm 0.01$ & 1.5 & $1.50 \pm 0.01$ & 0.3 & $0.30 \pm 0.01$ \\
\hline
\end{tabular}

${ }^{a}$ Composition given here is the same as given in MIRD Pamphlet No. 5 except that all elements with atomic number greater than zinc are considered zinc.

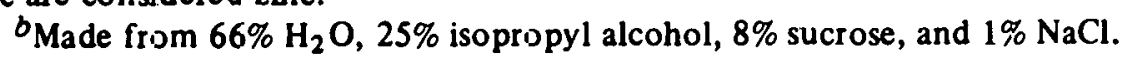

${ }^{c}$ Made from bone flour, water sucrose, and salts. (Ga74).

${ }^{d}$ Made from $73.5 \% \mathrm{H}_{2} \mathrm{O}, 25.0 \%$ cellulose (sponge), and $1.5 \% \mathrm{NaCl}$. 
Table 3. Calculated partial mass attenuation coefficients for Snyder-Fisher composition and comparison ${ }^{a}$ of equivalent material used in MR. ADAM phantom - soft tissue

\begin{tabular}{|c|c|c|c|c|c|c|}
\hline \multirow[b]{2}{*}{$\begin{array}{r}\text { Energy } \\
\cdot(\mathrm{keV})\end{array}$} & \multicolumn{2}{|c|}{ Photoelectric } & \multicolumn{2}{|c|}{ Coherent } & \multicolumn{2}{|c|}{ Compton } \\
\hline & $\begin{array}{l}\text { Coefficient } \\
\left(\mathrm{cm}^{2} / \mathrm{g}\right)\end{array}$ & $\begin{array}{c}\text { Comparison } \\
(\%)\end{array}$ & $\begin{array}{l}\text { Coefficient } \\
\left(\mathrm{cm}^{2} / \mathrm{g}\right)\end{array}$ & $\begin{array}{c}\text { Comparison } \\
(\%)\end{array}$ & $\begin{array}{l}\text { Coefrĩcient } \\
\left(\mathrm{cm}^{2} / \mathrm{g}\right)\end{array}$ & $\begin{array}{c}\text { Comparison } \\
(\%)\end{array}$ \\
\hline 10 & 4.34 & 1.7 & 0.231 & 1.7 & 0.153 & -0.7 \\
\hline 15 & 1.18 & 1.5 & 0.132 & 1.6 & 0.176 & -0.8 \\
\hline 20 & 0.465 & 1.4 & 0.0862 & 1.6 & 0.136 & -0.9 \\
\hline 30 & 0.124 & 1.1 & 0.0513 & 1.6 & 0.192 & -0.9 \\
\hline 40 & 0.0485 & 1.0 & 0.0277 & 1.5 & 0.190 & -0.9 \\
\hline 50 & 0.0235 & 0.9 & 0.0187 & 1.5 & 0.185 & -0.8 \\
\hline 60 & 0.0130 & 0.8 & 0.0135 & 1.5 & 0.180 & -0.8 \\
\hline 80 & $5.12 E-3$ & 0.7 & $7.93 E-3$ & 1.5 & 0.169 & -0.7 \\
\hline 100 & $2.50 \mathrm{E}-3$ & 0.6 & $5.20 E-3$ & 1.4 & 0.160 & -0.7 \\
\hline 150 & $6.96 \mathrm{E}-4$ & 0.4 & $2.37 \mathrm{E}-3$ & 1.4 & 0.141 & -0.6 \\
\hline 200 & $2.85 E-4$ & 0.3 & $1.34 \mathrm{E}-3$ & 1.5 & 0.12 .8 & -0.6 \\
\hline 300 & $8.40 E-5$ & 0.1 & $5.91 E-4$ & 1.5 & 0.111 & -0.5 \\
\hline 400 & $3.62 E-5$ & 0.1 & $3.30 E-4$ & 1.5 & 0.100 & -0.6 \\
\hline 500 & $1.92 E-5$ & 0.3 & $2.09 E-4$ & 1.6 & 0.0926 & -0.6 \\
\hline 600 & $1.15 E-5$ & 0.4 & $1.44 \mathrm{E}-4$ & 1.6 & 0.0871 & -0.6 \\
\hline 800 & $5.36 \mathrm{E}-6$ & 0.7 & $8.05 E-5$ & 1.7 & 0.0798 & -0.8 \\
\hline 1000 & $3.01 E-6$ & 0.9 & $5.13 E-5$ & 1.8 & 0.0751 & -0.9 \\
\hline
\end{tabular}

${ }^{a}$ Percent comparison of coefficients for equivalent material calculated as

$$
(\mu / \rho)_{\text {equivalene }}-(\mu / \rho)_{\text {Snyder-Fisher }}
$$

$190 \times \frac{(\mu / \rho)_{\text {Snyder-Fisher }}}{(\mu / \rho)^{2}}$ 
Table 4. Calculated partial mass attenuation coefficien ts for Snyder-Fisher composition and comparison ${ }^{a}$ of equivalent material used in MR. ADAM

phantom - skeleton

\begin{tabular}{|c|c|c|c|c|c|c|}
\hline \multirow[b]{2}{*}{$\begin{array}{r}\text { Energy } \\
(\mathrm{keV})\end{array}$} & \multicolumn{2}{|c|}{ Photoelectric } & \multicolumn{2}{|c|}{ Coherent } & \multicolumn{2}{|c|}{ Compton } \\
\hline & $\begin{array}{l}\text { Coefficient } \\
\left(\mathrm{cm}^{2} / \mathrm{g}\right)\end{array}$ & $\begin{array}{c}\text { Comparison } \\
(\%)\end{array}$ & $\begin{array}{c}\text { Coefficient } \\
\left(\mathrm{cm}^{2} / \mathrm{g}\right)\end{array}$ & $\begin{array}{c}\text { Comparison } \\
(\%)\end{array}$ & $\begin{array}{l}\text { Coefficient } \\
\left(\mathrm{cm}^{2} / \mathrm{g}\right)\end{array}$ & $\begin{array}{c}\text { Comparison } \\
(\%)\end{array}$ \\
\hline 10 & 15.4 & -1.2 & 0.327 & 1.5 & 0.143 & -1.2 \\
\hline 15 & 4.62 & -0.9 & 0.192 & 1.4 & 0.164 & -0.9 \\
\hline 20 & 1.93 & -0.8 & 0.126 & 1.4 & 0.174 & -0.8 \\
\hline 30 & 0.554 & -0.6 & 0.0671 & 1.4 & 0.181 & -0.6 \\
\hline 40 & 0.267 & -0.5 & 0.0416 & 1.4 & 0.179 & -0.5 \\
\hline 50 & 0.113 & -0.5 & 0.0282 & 1.4 & 0.176 & -0.5 \\
\hline 60 & 0.0641 & -0.5 & 0.0204 & 1.3 & 0.172 & -0.5 \\
\hline 80 & 0.0261 & -0.5 & 0.0120 & 1.3 & 0.162 & -0.5 \\
\hline 100 & 0.0131 & -0.5 & $7.90 E-3$ & 1.3 & 0.154 & -0.5 \\
\hline 150 & $3.76 E-3$ & -0.6 & $3.61 E-3$ & 1.3 & 0.138 & -0.6 \\
\hline 200 & $1.57 \mathrm{E}-3$ & -0.6 & $2.04 E-3$ & 1.3 & 0.126 & -0.6 \\
\hline 300 & $4.73 E-4$ & -0.6 & $9.02 E-4$ & 1.3 & 0.109 & -0.6 \\
\hline 400 & $2.06 E-4$ & -0.6 & $5.02 E-4$ & 1.4 & 0.0981 & -0.6 \\
\hline 500 & $1.10 E-4$ & -0.6 & $3.18 E-4$ & 1.4 & 0.0905 & -0.6 \\
\hline 600 & $6.66 E-5$ & -0.5 & $2.19 E-4$ & 1.4 & 0.0848 & -0.5 \\
\hline 800 & $3.08 E-5$ & -0.5 & $1.22 \mathrm{E}-4$ & 1.4 & 0.0768 & -0.5 \\
\hline 1000 & $1.73 E-5$ & -0.4 & $7.80 E-5$ & 1.5 & 0.0715 & -0.4 \\
\hline
\end{tabular}

${ }^{a}$ Percent comparison of coefficients for equivalent material calculated as

$$
(\mu / \rho)_{\text {equivalent }}-(\mu / \rho)_{\text {Snyder-Fisher }}
$$


Table 5. Calcislated partial mass attenuation coefficients for Snyder-Fisher composition and comparison ${ }^{a}$ of equivalent material used in MR. ADAM phantom - lung

\begin{tabular}{|c|c|c|c|c|c|c|}
\hline \multirow[b]{2}{*}{$\begin{array}{r}\text { Energy } \\
(\mathrm{keV})\end{array}$} & \multicolumn{2}{|c|}{ Photoelectzic } & \multicolumn{2}{|c|}{ Coherent } & \multicolumn{2}{|c|}{ Compton } \\
\hline & $\begin{array}{l}\text { Coeffizient } \\
\left(\mathrm{cm}^{2} / \mathrm{g}\right)\end{array}$ & $\begin{array}{c}\text { Comparison } \\
(\%)\end{array}$ & $\begin{array}{l}\text { Coefficient } \\
\left(\mathrm{cm}^{2} / \mathrm{g}\right)\end{array}$ & $\begin{array}{c}\text { Comparison } \\
(\%)\end{array}$ & $\begin{array}{l}\text { Coeffjcient } \\
\left(\mathrm{cm}^{2} / \mathrm{g}\right)\end{array}$ & $\begin{array}{c}\text { Comparison } \\
(\%)\end{array}$ \\
\hline 10 & 4.85 & 1.4 & 0.246 & 1.0 & 0.152 & -0.8 \\
\hline 15 & 1.33 & 1.3 & 0.141 & 1.0 & 0.176 & -0.7 \\
\hline 20 & 0.524 & 1.2 & 0.0918 & 1.0 & 0.186 & -0.6 \\
\hline 30 & 0.140 & 1.0 & 0.0480 & 1.0 & 0.192 & -0.5 \\
\hline 40 & 0.0549 & 0.8 & 0.0295 & 1.0 & 0.190 & -0.5 \\
\hline 50 & 0.0266 & 0.7 & 0.0199 & 1.0 & 0.185 & -0.5 \\
\hline 60 & 0.0147 & 0.6 & 0.0143 & 1.0 & 0.180 & -0.5 \\
\hline 80 & $5.81 \mathrm{E}-3$ & 0.5 & $8.43 E-3$ & 1.0 & 0.167 & -0.4 \\
\hline 100 & $2.85 E-3$ & 0.4 & $5.52 E-3$ & 1.0 & $0.16 \mathrm{~J}$ & -0.4 \\
\hline 150 & $7.91 \mathrm{E}-4$ & 0.2 & $2.51 E-3$ & 1.1 & 0.141 & -0.4 \\
\hline 200 & $3.25 \mathrm{E}-4$ & 0.1 & $1.42 E-3$ & 1.1 & 0.123 & -0.4 \\
\hline 300 & $9.57 E-5$ & 0.1 & $6.29 E-4$ & 1.1 & 0.111 & -0.4 \\
\hline 400 & $4.13 E-5$ & 0.2 & $3.51 \mathrm{E}-4$ & 1.1 & 0.100 & -0.4 \\
\hline 500 & $2.19 E-5$ & 0.3 & $2.23 E-4$ & 1.1 & 0.0926 & -0.4 \\
\hline 600 & $1.32 \mathrm{E}-5$ & 0.4 & $1.54 E-4$ & 1.1 & $0.08 ? 2$ & -0.4 \\
\hline 800 & $6.10 E-6$ & 0.5 & $8.60 E-5$ & 1.1 & 0.0798 & -0.4 \\
\hline 1000 & $3.43 \mathrm{E}-6$ & 0.6 & $5.49 E-5$ & 1.2 & 0.0751 & -0.4 \\
\hline
\end{tabular}

${ }^{a}$ Percent comparison of coefficier:ts for equivalent material calculated as

$$
100 \times \frac{(\mu / \rho) \text { equivalent }-(\mu / \rho) \text { Snyder-Fisher }}{(\mu / \rho)_{\text {Snyder-Fisher }}}
$$




\section{CHAPTER IV}

\section{RESULTS AND CONCLUSIONS}

\section{Outline of Spectral Measurements}

The data, which comprise the body of the results of this project, were collected and processed as shown schematically in Figure 21 . The spectrum measurements presented in this chapter were performed in a period of ten days. To ensure that long term drifts in the electronic equipment did not bias the data, the performance of the data collection system was checked daily. Pulse height spectra were collected for two sources $\left({ }^{57} \mathrm{Co}\right.$ and $\left.{ }^{241} \mathrm{Am}\right)$. If necessary, adjustments were made to the gain of the linear amplifier to ensure that the modal values of the pulse height distributions were observed in the proper channel.

The $\mathrm{NaI}(\mathrm{T} \ell)$ probe was positioned at seven different locations within or on the surface of the MR. ADAM phantom. At each probe location pulse height spectra were acquired for twenty-one combinations of seven beam configurations and three generating potentials $(60,80,100 \mathrm{kV} c \mathrm{p})$. In each case, the $x$-ray machine tube current was adjusted so that the multichannel analyzer was operating with a dead time of $10 \%$ or less. Tube currents used ranged from $20 \mathrm{~mA}$ to $0.1 \mu \mathrm{A}$. The analyzer was equipped with circuitry to correct for dead time, and this feature was used in acquiring all spectra. For each case, data were collected long enough to provide 50,000 to 100,000 counts per channel in the modal channel. Counting times of 100 seconds live time were used most 


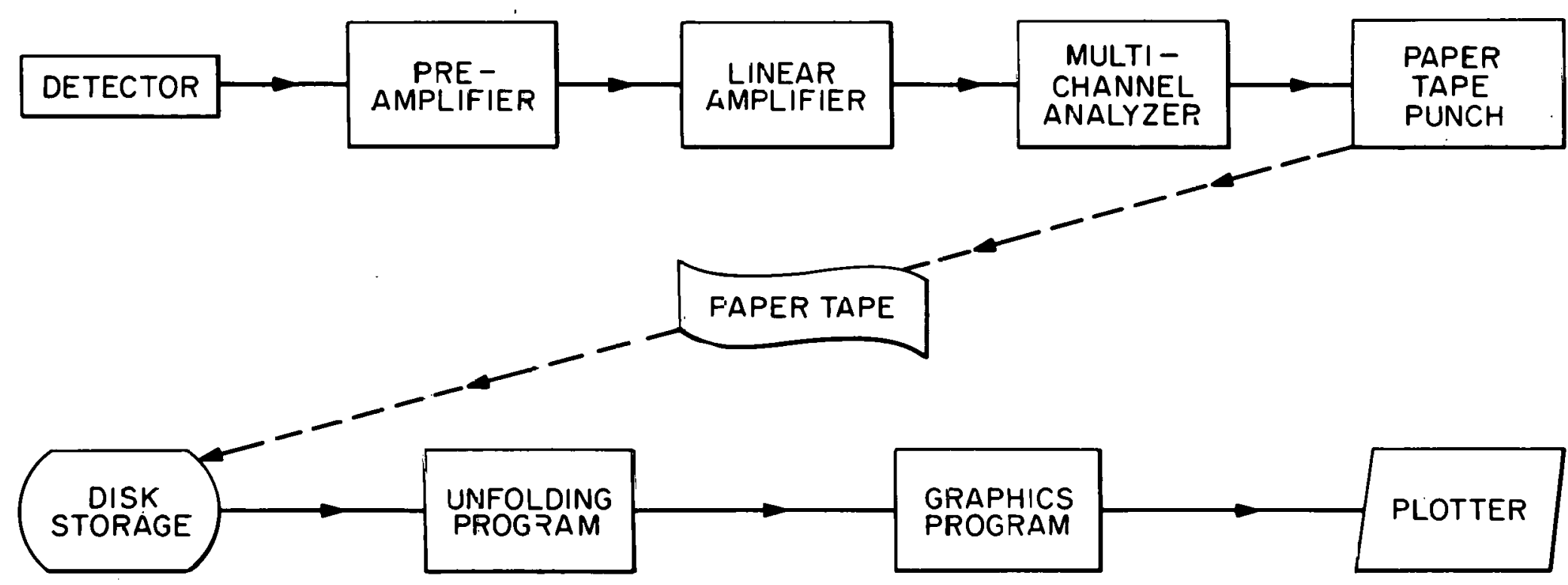

Figure 21. Daté Acquisition and Processing Schematic 
frequently, but occasionally counting times up to 500 seconds live time were necessary. Before each pulse height spectrum was punched on tape, all important parameters such as generating potential, tube current, detector location, etc. were punched on the tape manually to facilitate subsequent data processing.

The seven probe locations were chosen to be at the center of or near seven regions of interest: the testes, the right ovary, the thyroid, the eyes, the heart, the fifth lumbar vertebra and the thoracic region of the spine. Testes, ovary, thyroid and eyes were chosen because of their radiological significance. The location near the heart was chosen to provide data for this organ and for other organs located nearby. The two locations in the spine were chosen to provide data for at least one of the skeletal structures containing red bone marrow.

A rectilinear coordinate system whose origin is located at the center of the base of the torso section of the phantom was used to determine the coordinates of the probe locations and the organs. The coordinates of the centers of the organs chosen and the coordinates and reference numbers of the corresponding probe locations are given in Table 6. Figure 22 shows the $x$-axis and $z$-axis of the coordinale system. The $y$-axis is perpendicular to the $x-z$ plane shown, and the positive $y$-direction is toward the posterior of the phantom such that the coordinate system is right-handed.

The triangles in Figure 22, numbered 1 through 7 for crossreference with Table 6 , show the location and approximate orientation of the probe within the phantom. The vertex of the acute angle of each triangle shows the position of the active volume of the detector. The 
Table 6. Summary of Probe Locations

\begin{tabular}{clll}
\hline $\begin{array}{c}\text { Reference } \\
\text { Number }\end{array}$ & Organ or Region & $\begin{array}{c}\text { Coordinates of Probe } \\
(\mathrm{X}, \mathrm{Y}, \mathrm{Z})\end{array}$ & $\begin{array}{c}\text { Coordinates of Center } \\
\text { of Organ } \\
(\mathrm{X}, \mathrm{Y}, \mathrm{Z})\end{array}$ \\
\hline 1 & Testes & $-2.0,-7.2,-3.0$ & $-1.3,-8.0,-2.3$ \\
2 & Right Ovary & $-6.0,0,15.0$ & $-6.0,0,15.0$ \\
3 & Fifth Lumbar Vertebra & 0, center of spine, 23.0 & $*$ \\
4 & Heart Region & $0,0,44.0$ & $1.0,-3.0,51.0$ \\
5 & Thcracic Spine & 0, center of spine, 47.0 & $*$ \\
6 & Thyroid Gland & $0,-6.0,72.5$ & $0,-6.0,72.5$ \\
7 & Eye Region & 0, front of head, 84.0 & 0, front of head, 83.5 \\
\hline
\end{tabular}

${ }^{\dagger}$ As specified in Snyder-Fisher Phantom.

* Not specifically defined in Snyder-Fisher Phantom. 


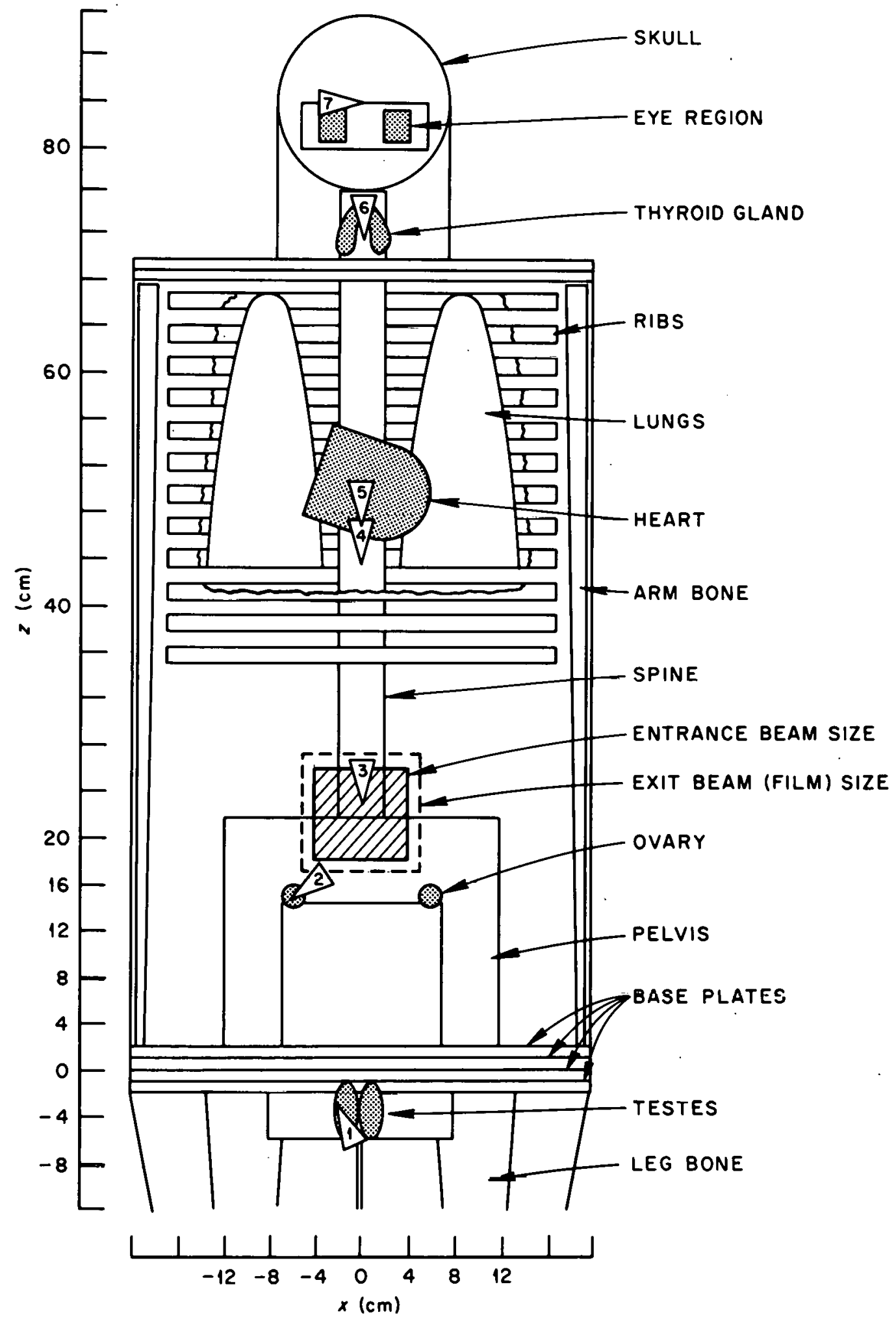

Figure 22. Beam Location for Lower Spine $10 \times 10 \mathrm{~cm}$ Field (Center at $x=0, z=22$ ). 
base of each triangle indicates the direction of the photomultiplier tube housing part of the detector. Note that the triangles do not show the full length of the region occupied by the probe. (The probe was over $20 \mathrm{~cm}$ long, half the width of the phantom.) The detector was oriented parallel to the $x-z$ plane for the eye, heart, and two spine regions. In the right ovary location the probe was positioned with its base toward the posterior of the phantom (iniclined approximately $30^{\prime}$ to the $x-z$ plane). In the testes location the probe was positioned with its base toward the anterior (inclined approximately $15^{\circ}$ to the $x-z$ plane). The probe was positioned at the thyroid location by placing it parallel to the $y-z$ plane inclined toward the posterior approximately $60^{\circ}$ from the $z$-axis.

To facilitate positioning the probe, the configuration of the phantom was changed in some cases, and a number of methods were used to hold the probe. A device was built to hold the probe at the heart and right ovary locations. The device, built mostly of nylon and lucite, consisted of an $x-y$ translator that was bolted to the top of the torso and a $1 \mathrm{~cm}$ square polyester rod that provided z-direction movement. When this device was used, the head section of the phantom was not emplaced. The detector was held in the spine by means of two nylon collars that were fastened to the photomultiplier tube housing. In this case the head was not used also. The detector was positioned at the eye, thyroid and testes with a variety of small clamps and rods. The skull was removed when thyroid measurements were made. Entrance to the testes region was accomplished via a latex covered hole in the base of the male genitalia region. 
In all cases care was taken to minimize the "shielding" of the Phantom or the probe with parts of the positioning apparatus. The sizes of the phantom and the detector prohibited placing the detector in a desired attitude directly at the center of the heart and the testes. The detector was not positioned at the center of the eye region because of slight discrepancies between the shape of the heads of the SnyderFisher phantom and the MR. ADAM phantom.: Laser beams, a plumb bob, and a meter stick were used to determine accurately the position of the detector within the phantom. It was estimated that the overall positioning accuracy was $\pm 0.3 \mathrm{~cm}$.

Beam sizes and locations simulating $x$-ray examinations of the chest, left shoulder, upper gastro-intestinal (upper G.I.), pelvic and two spinal regions were selected. Several factors were considered in choosing the particular radiological examinations to be simulated. It was desired to choose a set of exposure configurations which would be both representative of the most frequent medical $x$-ray examinations and yet not redundant. For example, the pelvic exam and an abdominal exam were selected, but a pyelogram, which might be centered slightly differently but in between the two, was not. Also examinations of the head and extremities were not chosen because of the phantom's over-simplified geometry in these regions. The abdominal and two spinal configurations were chosen to have the same beam center so that the effect of field size could be studied.

Generally anatomical landmarks such as the xiphoid process, the bi-iliac crest, and the pubic symphysis are used in positioning $x$-ray beams with respect to a human patient. Since there was some uncertainty 
in ascertaining the locations on the phantom that corresponded to the landmarks, there was some difficulty in selecting the beam configurations. However, with the help of an $x$-ray technologist, the seven beam sizes and positions were defined.

All examinations were simulated with the phantom standing erect. A simulated focal-spot-to-film distance of $120 \mathrm{~cm}$ was chosen because the collimator would not open wide enough to accommodate the largest beam size $(36 \times 44 \mathrm{~cm})$ at the more conventional distannre of $102 \mathrm{~cm}$ (40 inches). For each simulated examination the collimator and the tube height were adjusted until the beam was positioned and sized appropriately at the plane that would be occupied by the $f i l m$ in a real $x$-ray examination. The outline of the beam where it entered the phantom was marked for subsequent use.

The beam sizes and locations are pictured in Figures 22-28. Note that the shaded area shows the outline of the beam on the anterior of the phantom and that the dotted lines show the outline on the posterior. Figure 28 shows the configuration for a chest $x$-ray which had the beam incident on the posterior of the phantom.

In addition to the 147 combinations of detector locatiun, bcam configuration, and generating potential described above, several other configurations were employed. Spectra were acquired at the testes with the male genitalia region shielded with a $10 \times 15 \mathrm{~cm}$ sheet of lead $0.16 \mathrm{~cm}$ thick. For the shoulder exam spectra were acquired at the thyroid with the neck shielded with a similar sheet of lead covering the front half of the neck region. The locations of the testes and thyroid shield are shown in Figure 26. Also for the shoulder exam, additional measure- 


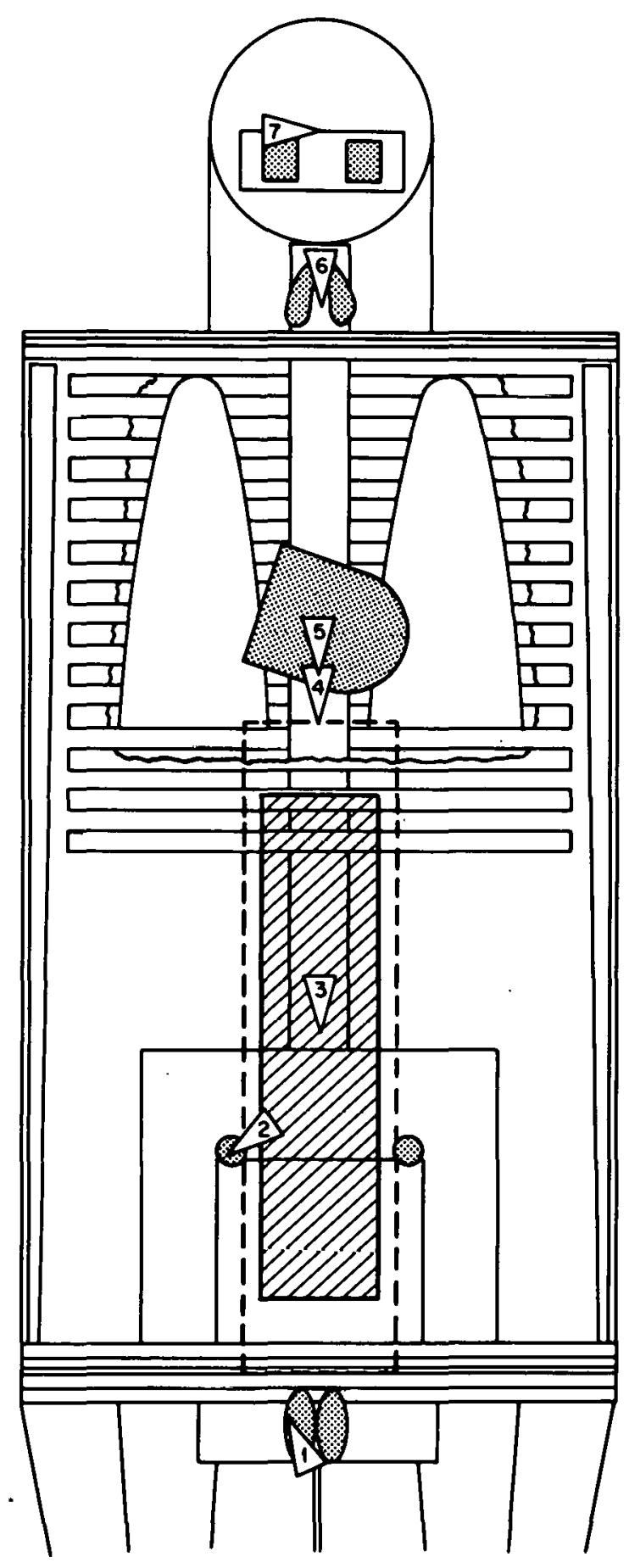

Figure 23. Beam Location for Full Spine $10 \times 44 \mathrm{~cm}$ Field (Center at $x=0, z=22$ ). 


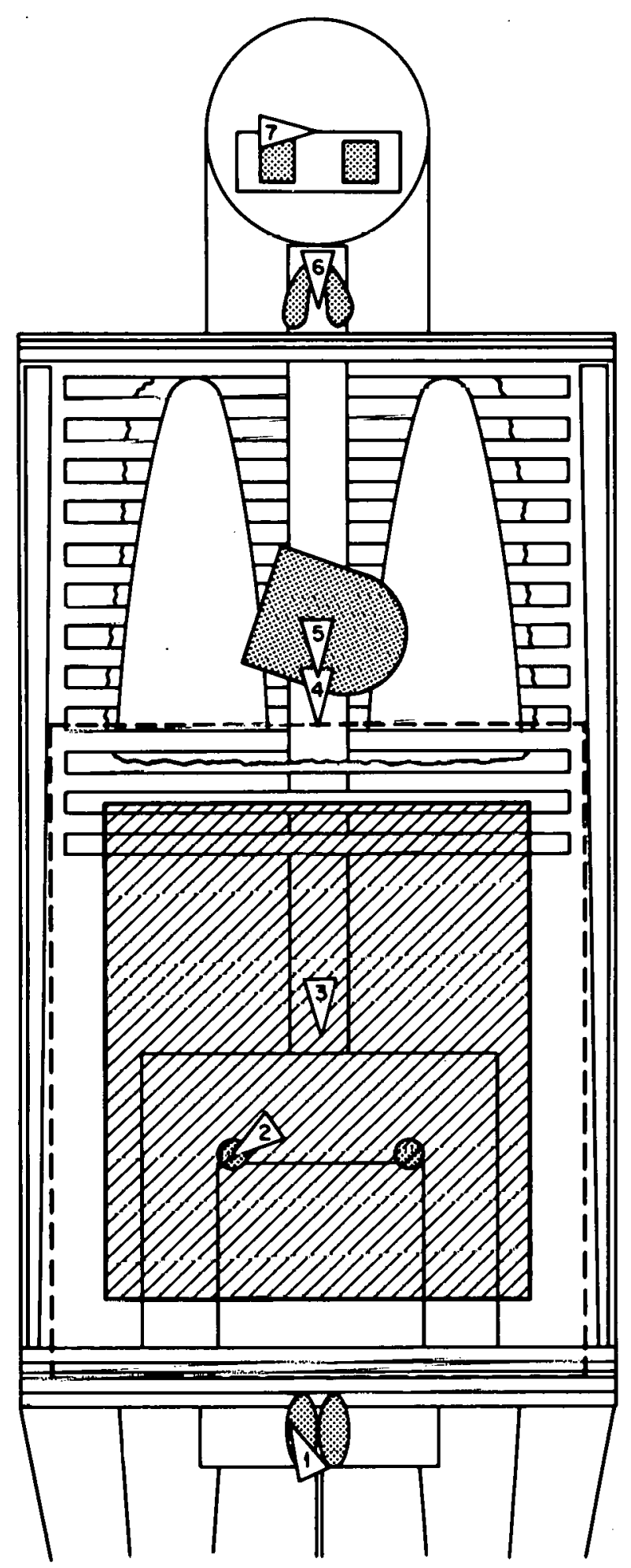

Figure 24. Beam Location for Abdominal $36 \times 44 \mathrm{~cm}$ Field (Center at $x=0, z=22$ ). 


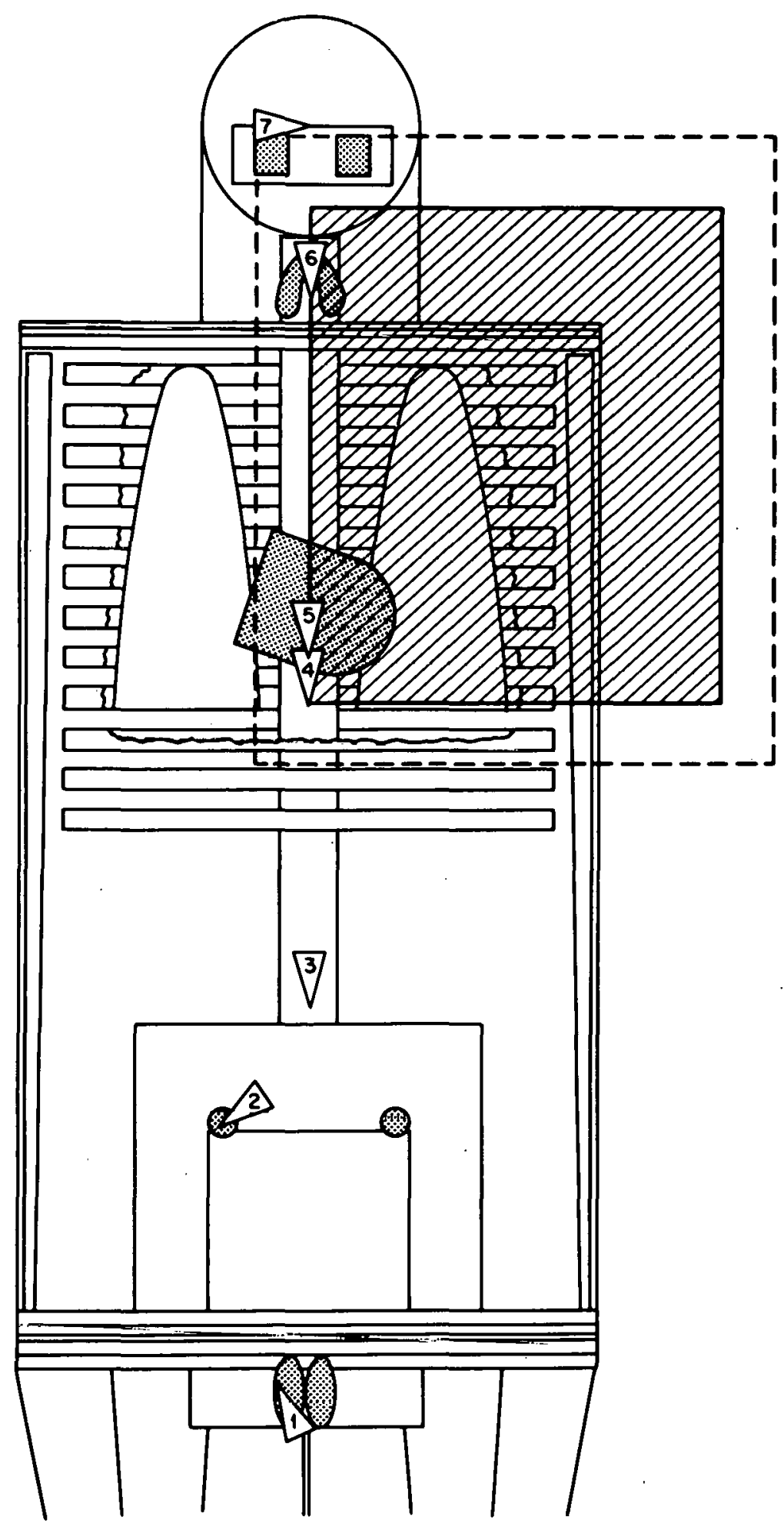

Figure 25. Beam Location for Shoulder $36 \times 44 \mathrm{~cm}$ Field (Center at $x=14.3, z=61.5)$. 


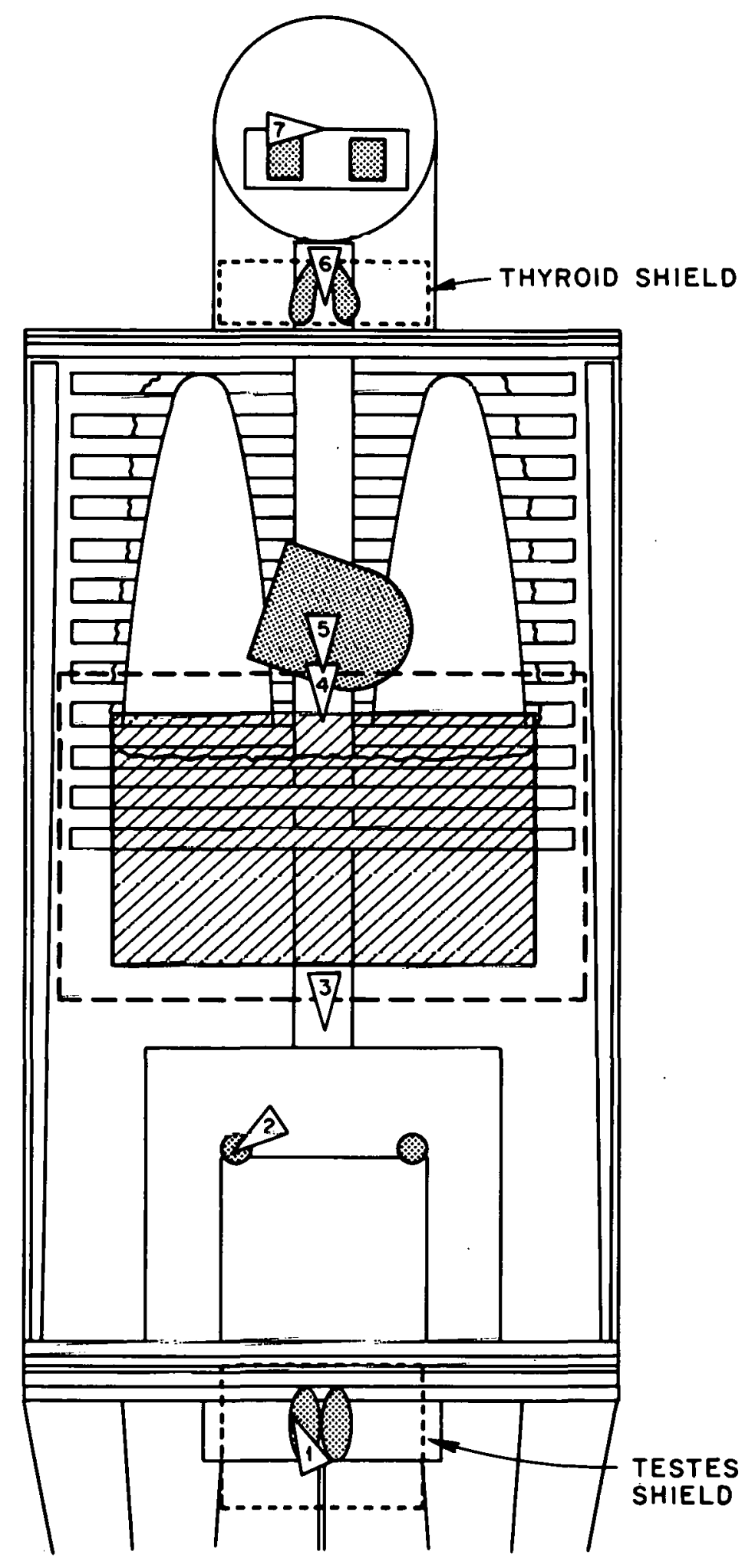

Figure 26. Beam Location for Upper Gastro-Intestinal (Upper G.I.) $36 \times 22 \mathrm{~cm}$ Field (Center at $x=0, z=36$ ). 


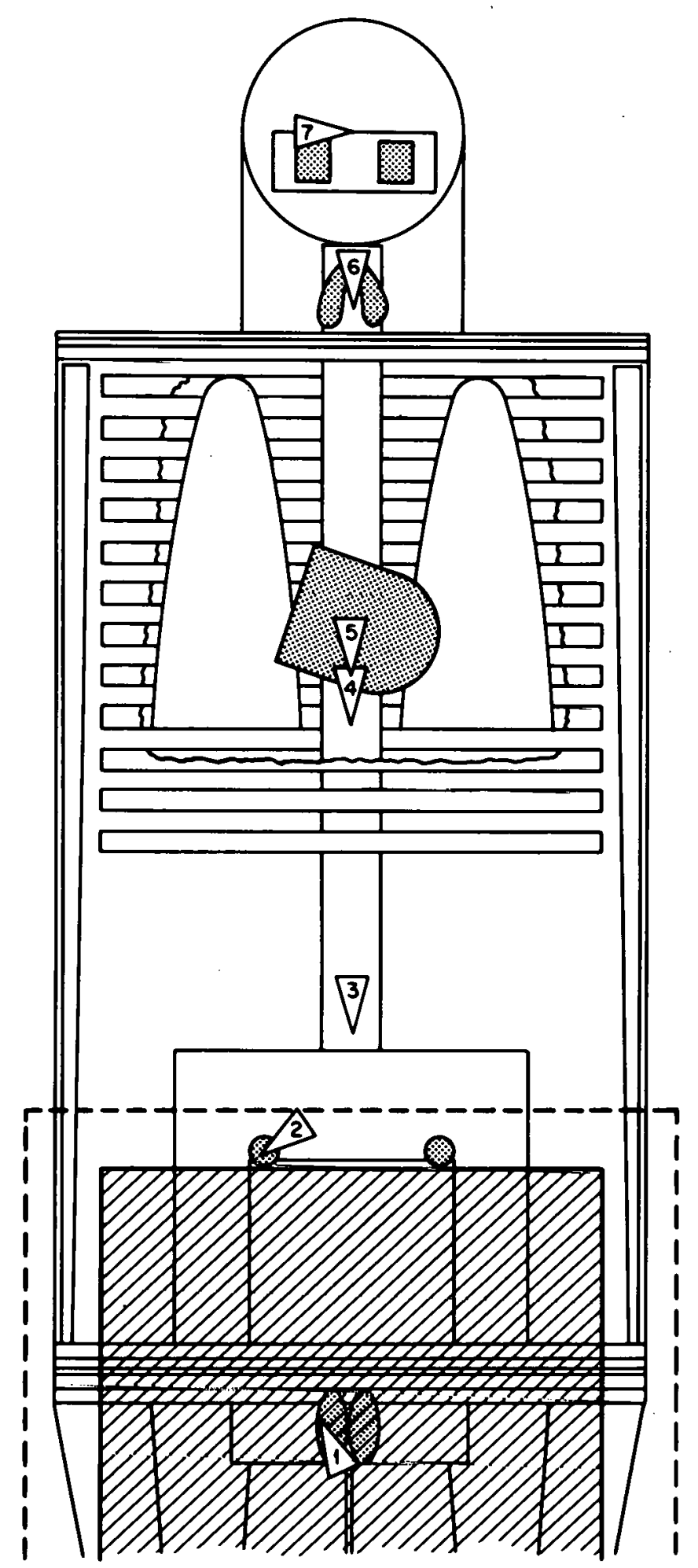

Figure 27. Beam Location for Pelvic $44 \times 36 \mathrm{~cm}$ Field (Center at $x=z=0)$. 


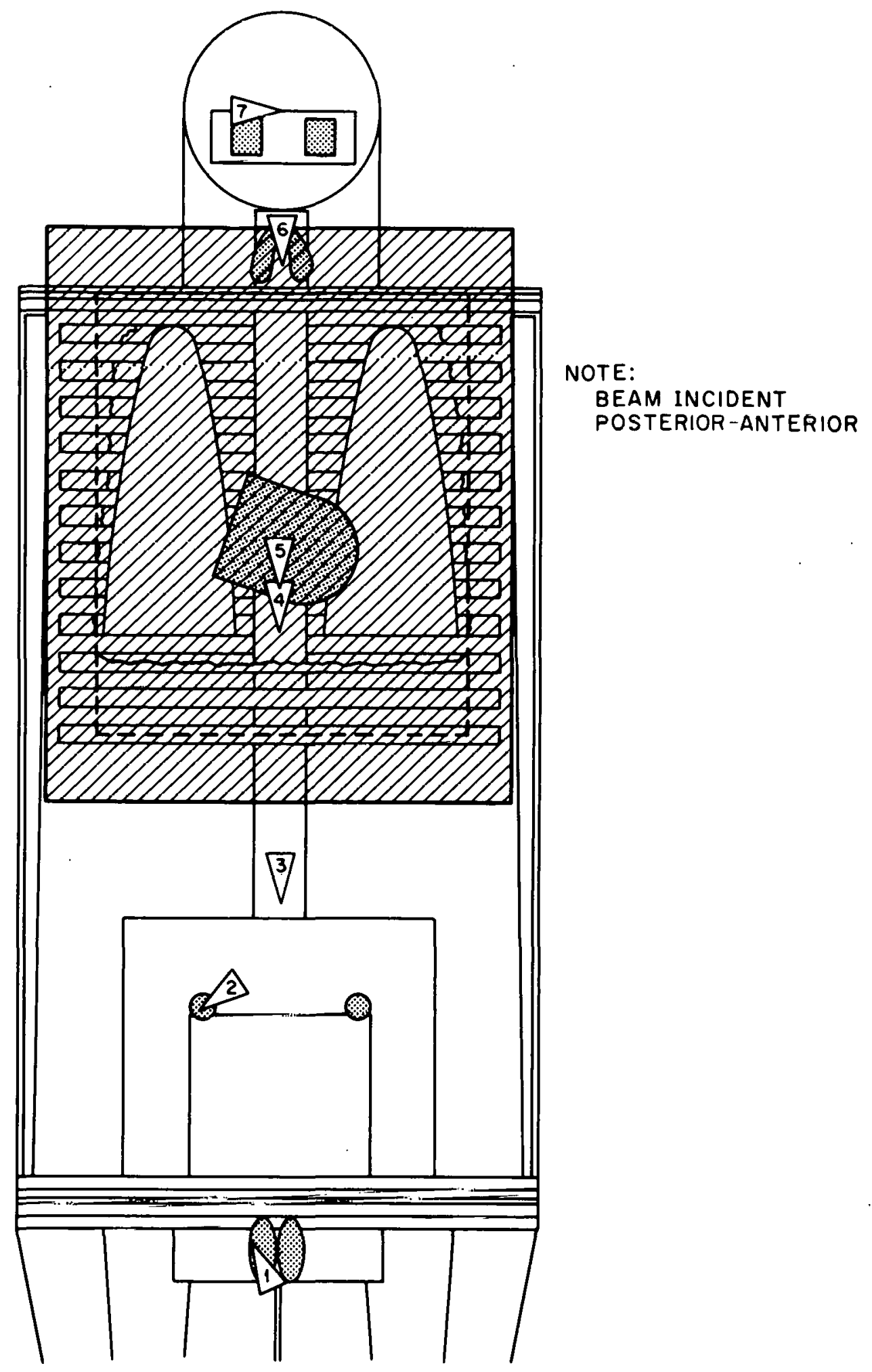

Figure 28. Beam Location for Chest Posterior-Anterior $36 \times 44 \mathrm{~cm}$ Field (Center at $x=0, z=53$ ). 
ments were made at the eyes and thyroid with a beam size slightly smaller than otherwise employed. For the upper G.I., shoulder, and chest exams, measurements at the right ovary were made with the phantom shielded with an apron of $0.16 \mathrm{~cm}$ thick lead. When used, the apron was placed just below the edge of the beam and was large enough to completely cover the lower half of the torso. Further special measurements in and around the heart region were made for a number of $10 \times 10 \mathrm{~cm}$ beams incident nearby. These special configurations will be explained in the next section.

After the program of measurements outlined above was completed, the pulse height distributions were unfolded and graphed. The results were normalized per unit tube current and per unit time. (Tube current was expressed in milliamps in this text because of its traditional acceptance in the field of diagnostic radiology). As the data were processed the centroid and the normalized dose rate [calculated from the unfolded spectra with Equation (3-6)] were calculated. The centroid and dose rates for all exposure configurations are summarized in Tables 7-9. The unfolded spectra are presented graphically in Appendix A.

\section{Highlights of Results}

\section{Protective Measures}

The effect of gonad shielding on the absorbed dose rate and the spcctrum at the testes was investigated. As was explained previously, pairs of spectra were collected in identical manners except that in one of each pair a testes shield was used. The spectra and absorbed dose rates for the pelvic, abdominal and upper G.I. exams for 60 and $80 \mathrm{kVcp}$ are given in Figures $29-31$. The approximately $90 \%$ reduction in absorbed 
Table 7. Summary of Spectral Data for Testes and Right Ovary

\begin{tabular}{|c|c|c|c|c|c|c|c|c|c|}
\hline \multirow{2}{*}{$\begin{array}{l}\text { Detector } \\
\text { Location }\end{array}$} & \multirow{2}{*}{ Beam Type } & \multirow{2}{*}{$\begin{array}{l}\text { Size } \\
(\mathrm{cm})\end{array}$} & \multirow[b]{2}{*}{ Notes ${ }^{a}$} & \multicolumn{2}{|c|}{$60 \mathrm{kV} c p$} & \multicolumn{2}{|c|}{$80 \mathrm{kVcp}$} & \multicolumn{2}{|c|}{$100 \mathrm{kVcp}$} \\
\hline & & & & $\mathrm{D}^{b}$ & Centroid $^{c}$ & $\mathrm{D}$ & Centroid & $\mathrm{D}$ & Centroid \\
\hline \multirow[t]{13}{*}{ Testes } & Lower Spine & $10 \times 10$ & & $4.37 \mathrm{E}-4^{d}$ & 38 & $1.59 \mathrm{E}-3$ & 45 & $3.88 \mathrm{E}-3$ & 51 \\
\hline & & & $w / s$ & $3.06 \mathrm{E}-4$ & 38 & $1.30 \mathrm{E}-3$ & 45 & $3.43 \mathrm{E}-3$ & 51 \\
\hline & Full Spine & $10 \times 44$ & & $1.97 \mathrm{E}-2$ & 37 & $5.53 \mathrm{E}-2$ & 44 & $1.17 \mathrm{E}-1$ & 51 \\
\hline & & & $w / s$ & $1.06 \mathrm{E}-2$ & 38 & $3.37 \mathrm{~F}-\mathrm{-}$ & 45 & $7.74 \mathrm{E}-2$ & $\mathfrak{j 1}$ \\
\hline & Abdominal & $36 \times 44$ & & $4.35 E-2$ & 37 & $1.36 \mathrm{E}-1$ & 45 & $2.99 \mathrm{E}-1$ & 51 \\
\hline & & & $w / s$ & $2.30 \mathrm{E}-2$ & 38 & $9.71 E-2$ & 45 & $2.30 \mathrm{E}-1$ & 51 \\
\hline & Shoulder & $36 \times 44$ & & $3.55 \mathrm{E}-4$ & 36 & $1.03 \mathrm{E}-3$ & 44 & $2.14 \mathrm{E}-3$ & 50 \\
\hline & & & $w / s$ & $2.57 \mathrm{E}-4$ & 37 & $7.92 \mathrm{E}-4$ & 45 & $1.68 \mathrm{E}-3$ & 51 \\
\hline & Upper G.I. & $36 \times 22$ & & $5.93 \mathrm{E}-4$ & 37 & $1.69 \mathrm{E}-3$ & 45 & $3.98 \mathrm{E}-3$ & 51 \\
\hline & & & $w / s$ & $2.99 \mathrm{E}-4$ & 37 & $1.06 \mathrm{E}-3$ & 45 & $2.68 \mathrm{E}-3$ & 51 \\
\hline & Pelvic & $44 \times 36$ & $\dagger$ & 2.02 & 37 & 4.27 & 45 & 7.70 & 52 \\
\hline & & & $w / s \dagger$ & $2.21 \mathrm{E}-1$ & 37 & $6.56 \mathrm{E}-1$ & 44 & 1.38 & 50 \\
\hline & Chest & $36 \times 44$ & PA & $1.03 \mathrm{E}-4$ & 37 & $4.24 \mathrm{E}-4$ & 45 & $1.23 \mathrm{E}-3$ & 51 \\
\hline \multirow[t]{10}{*}{ Right Ovary } & Lower Spine & $10 \times 10$ & & $1.20 \mathrm{E}-2$ & 38 & $4.57 \mathrm{E}-2$ & 46 & $1.06 \mathrm{E}-1$ & 52 \\
\hline & Full Spine & $10 \times 44$ & & $5.21 \mathrm{E}-2$ & 37 & $2.08 \mathrm{E}-1$ & 46 & $4.56 \mathrm{E}-1$ & 52 \\
\hline & Abdominal & $36 \times 44$ & $\dagger$ & $3.48 \mathrm{E}-1$ & 39 & $9.98 \mathrm{E}-1$ & 48 & 2.29 & 55 \\
\hline & Shoulder & $36 \times 44$ & & $3.12 \mathrm{E}-4$ & 38 & $1.18 \mathrm{~F}-3$ & 46 & $3: 06 \mathrm{E}-3$ & 51 \\
\hline & & & w/A & $6.11 \mathrm{E}-5$ & 38 & $3.57 \mathrm{E}-4$ & 46 & $1.15 \mathrm{E}-3$ & 50 \\
\hline & Upper U..I. & $36 \times 22$ & & $6.63 \mathrm{E}-3$ & 38 & $2.62 \mathrm{E}-2$ & 46 & $6.78 E-2$ & 51 \\
\hline & & & $w / A$ & $4.08 \mathrm{E}-3$ & 38 & $1.78 \mathrm{E}-2$ & 46 & $4.77 \mathrm{E}-2$ & 51 \\
\hline & Pelvic & $44 \times 36$ & $\dagger$ & $1.50 \mathrm{E}-1$ & 39 & $6.45 \mathrm{E}-1$ & 48 & 1.45 & 56 \\
\hline & Chest & $36 \times 44$ & $\mathrm{FA}$ & $1.32 \mathrm{E}-3$ & $3 y$ & $5.70 \mathrm{E}-3$ & 46 & $1.54 \mathrm{E}-2$ & 52 \\
\hline & & & PA w/A & $5.62 \mathrm{E}-4$ & 39 & $3.20 \mathrm{E}-3$ & 46 & $8.68 \mathrm{E}-3$ & 51 \\
\hline
\end{tabular}

$a_{w / s}$ indicates that testes were shielded with a $10 \times 15 \mathrm{~cm}$ piece of lcad $0.16 \mathrm{~cm}$ thick.

w/A indicates that an apron of lead $0.16 \mathrm{~cm}$ thick was used to cover the lower torso. The upper edge of the apron abutted the lower edge of the beam.

$\dagger$ indicates that the detector was located in the primary beam.

PA indicates that the beam was incident posterior-anterinr

${ }^{b_{\mathrm{D}}}$ is the absorbed dose rate per unit tube current. It has units of $\mathrm{mrad} / \mathrm{sec}-\mathrm{mA}$.

${ }^{c}$ The controid is tlie centrold of the tluence vs energy distribution. It has units of keV.

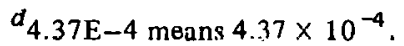


Table 8. Summary of Spectral Data for Heart Region, Thoracic Spine, and Fifth Lumbar Vertebra

\begin{tabular}{|c|c|c|c|c|c|c|c|c|c|}
\hline \multirow{2}{*}{$\begin{array}{l}\text { Detector } \\
\text { Location }\end{array}$} & \multirow{2}{*}{ Beam Type } & \multirow{2}{*}{$\begin{array}{l}\text { Size } \\
(\mathrm{cm})\end{array}$} & \multirow{2}{*}{ Notes $^{a}$} & \multicolumn{2}{|c|}{$60 \mathrm{kVcp}$} & \multicolumn{2}{|c|}{$80 \mathrm{kVcp}$} & \multicolumn{2}{|c|}{$100 \mathrm{kVcp}$} \\
\hline & & & & $\mathrm{D}^{b}$ & Centroid $^{c}$ & $\mathrm{D}$ & $\overline{\text { Centroid }}$ & D & Centroid \\
\hline \multirow[t]{7}{*}{ Heart Region } & Lower Spine & $10 \times 10$ & & $7.61 \mathrm{E}-4$ & 41 & $3.11 \mathrm{E}-3$ & 48 & $8.93 E-3$ & 54 \\
\hline & Full Spine & $10 \times 44$ & & $1.21 \mathrm{E}-2$ & 37 & $9.31 \mathrm{E}-2$ & 48 & $2.09 \mathrm{E}-1$ & 55 \\
\hline & Abdominal & $36 \times 44$ & & $2.64 \mathrm{E}-2$ & 37 & $1.91 \mathrm{E}-1$ & 48 & $4.54 \mathrm{E}-1$ & 55 \\
\hline & Shoulder & $36 \times 44$ & & $3.54 \mathrm{E}-2$ & 36 & $1.98 \mathrm{E}-1$ & 47 & $4.44 \mathrm{E}-1$ & 53 \\
\hline & Upper G.I. & $36 \times 22$ & $\dagger$ & $1.35 \mathrm{E}-1$ & 37 & $8.04 \mathrm{E}-1$ & 49 & 1.73 & 56 \\
\hline & Pelvic & $44 \times 36$ & & $2.33 \mathrm{E}-4$ & 36 & $1.93 \mathrm{E}-3$ & 47 & $5.08 \mathrm{E}-3$ & 54 \\
\hline & Chest & $36 \times 44$ & $\mathbf{P A} \dagger$ & $9.71 \mathrm{E}-2$ & 37 & $6.29 \mathrm{E}-1$ & 49 & 1.53 & 56 \\
\hline \multirow[t]{7}{*}{ Thoracic Spine } & Lower Spine & $10 \times 10$ & & $3.14 \mathrm{E}-5$ & 38 & $7.58 \mathrm{E}-4$ & 49 & $2.55 \mathrm{E}-3$ & 56 \\
\hline & Full Spine & $10 \times 44$ & & $1.27 \mathrm{E}-3$ & 38 & $2.17 \mathrm{E}-3$ & 51 & $6.45 \mathrm{E}-2$ & 58 \\
\hline & Abdominal & $36 \times 44$ & & $3.00 \mathrm{E}-3$ & 38 & $4.85 E-2$ & 50 & $1.34 \mathrm{E}-1$ & 57 \\
\hline & Shoulder & $36 \times 44$ & & $9.12 \mathrm{E}-2$ & 38 & $7.41 \mathrm{E}-2$ & 50 & $2.10 \mathrm{E}-1$ & 57 \\
\hline & Upper G.I. & $36 \times 22$ & & $1.35 \mathrm{E}-2$ & 38 & $1.36 \mathrm{E}-1$ & 51 & $3.90 \mathrm{E}-1$ & 59 \\
\hline & Pelvic & $44 \times 36$ & & $3.05 \mathrm{E}-5$ & 37 & $6.10 \mathrm{E}-4$ & 49 & $2.02 \mathrm{E}-3$ & 55 \\
\hline & Chest & $36 \times 44$ & $\mathbf{P A} \dagger$ & $2.39 \mathrm{E}-1$ & $\cdot 37$ & 1.42 & 50 & 3.26 & 57 \\
\hline \multirow{7}{*}{$\begin{array}{l}\text { Fifth Lumbar } \\
\text { Vertebra }\end{array}$} & Lower Spine & $10 \times 10$ & $\dagger$ & $1.13 \mathrm{E}-2$ & 39 & $1.10 \mathrm{E}-1$ & 52 & $2.82 \mathrm{E}-1$ & 60 \\
\hline & Full Spine & $10 \times 44$ & $\dagger$ & $1.53 \mathrm{E}-2$ & 38 & $1.64 \mathrm{E}-1$ & 51 & $4.25 \mathrm{E}-1$ & 58 \\
\hline & Abdominal & $36 \times 44$ & $\dagger$ & $2.54 \mathrm{E}-2$ & 38 & $2.71 \mathrm{E}-1$ & 50 & $4.48 \mathrm{E}-1$ & 56 \\
\hline & Shoulder & $36 \times 44$ & & $1.02 \mathrm{E}-4$ & 36 & $1.52 \mathrm{E}-3$ & 47 & $4.42 \mathrm{E}-3$ & 53 \\
\hline & Upper G.I. & $36 \times 22$ & & $2.49 \mathrm{E}-3$ & 37 & $3.40 \mathrm{E}-2$ & 48 & $9.01 \mathrm{E}-2$ & 54 \\
\hline & Pelvic & $44 \times 36$ & & $3.39 \mathrm{E}-3$ & 38 & $4.73 \mathrm{E}-2$ & 49 & $1.39 \mathrm{E}-1$ & 55 \\
\hline & Chest & $36 \times 44$ & PA & $1.66 \mathrm{E}-3$ & 36 & $1.41 \mathrm{E}-2$ & 47 & $3.56 \mathrm{E}-2$ & 53 \\
\hline
\end{tabular}

$a_{w / 3}$ indicates that testes were shielded with a $10 \times 15 \mathrm{~cm}$ piece of lead $0.16 \mathrm{~cm}$ thick. $\dagger$ indicates that the detector was located in the primary beam.

PA indicates that the beam was incident posterior-anterior.

${ }^{b} \mathrm{D}$ is the absorbed dose rate per unit tube current. It has units of $\mathrm{mrad} / \mathrm{sec}-\mathrm{mA}$.

${ }^{c}$ The centroid is the centroid of the fluence vs energy distribution. It has units of keV.

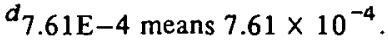


Table 9. Summary of Spectral Data for Thyroid Gland and Eye Region

\begin{tabular}{|c|c|c|c|c|c|c|c|c|c|}
\hline \multirow{2}{*}{$\begin{array}{l}\text { Detector } \\
\text { Location }\end{array}$} & \multirow{2}{*}{ Beam Type } & \multirow{2}{*}{$\begin{array}{l}\text { Size } \\
(\mathrm{cm})\end{array}$} & \multirow{2}{*}{ Notes $^{a}$} & \multicolumn{2}{|c|}{$60 \mathrm{kVcp}$} & \multicolumn{2}{|c|}{$80 \mathrm{kV} c p$} & \multicolumn{2}{|c|}{$100 \mathrm{kVcp}$} \\
\hline & & & & $\mathrm{D}^{b}$ & Centroid $^{c}$ & $\mathrm{D}$ & Centroid & D & Centroid \\
\hline \multirow[t]{7}{*}{ Thyroid Gland } & Lower Spine & $10 \times 10$ & & $1.65 \mathrm{E}-5$ & 36 & $9.70 \mathrm{E}-5$ & 46 & $2.34 E-4$ & 54 \\
\hline & Full Spine & $10 \times 44$ & & $1.37 E-4$ & 35 & $8.37 \mathrm{E}-4$ & 45 & $1.90 E-3$ & 52 \\
\hline & Aluduminal & $36 \times 44$ & & $5.07 E-4$ & 35 & $? .89 \mathrm{E}-3$ & 45 & $6.60 \mathrm{E}-3$ & 52 \\
\hline & Shoulder & $\begin{array}{l}36 \times 44 \\
36 \times 44 \\
28 \times 44\end{array}$ & $\begin{array}{l}\dagger_{\mathrm{w} / \mathrm{s}^{d} \dagger}{ }_{\text {Smaller }} \\
\text { beam }\end{array}$ & $\begin{array}{l}4.46 \mathrm{E}-1 \\
4.56 \mathrm{E}-2 \\
3.18 \mathrm{E}-2\end{array}$ & $\begin{array}{l}36 \\
35 \\
35\end{array}$ & $\begin{array}{l}2.00 \\
2.61 \mathrm{E}-1 \\
2.53 \mathrm{E}-1\end{array}$ & $\begin{array}{l}48 \\
46 \\
44\end{array}$ & $\begin{array}{l}3.79 \\
5.61 E-1 \\
5.10 E-1\end{array}$ & $\begin{array}{l}57 \\
53 \\
51\end{array}$ \\
\hline & Upper G.I. & $36 \times 22$ & & $5.34 \mathrm{E}-4$ & 35 & $3.62 \mathrm{E}-3$ & 45 & $8.56 \mathrm{E}-3$ & 52 \\
\hline & Pelvic & $44 \times 36$ & & $1.68 \mathrm{E}-4$ & 36 & $8.86 \mathrm{E} \cdot 4$ & 46 & $1.88 \mathrm{E}-3$ & 53 \\
\hline & Chest & $36 \times 44$ & $\mathbf{P A}$ & $1.60 \mathrm{E}-2$ & 37 & $1.61 \mathrm{E}-1$ & 49 & $3.55 \mathrm{E}-1$ & 55 \\
\hline \multirow[t]{7}{*}{ Eye Region } & Lower Spine & $10 \times 10$ & & $7.16 \mathrm{E}-5$ & 32 & $2.48 \mathrm{E}-4$ & 41 & $4.34 \mathrm{E} \cdot 1$ & 48 \\
\hline & Full Spine & $10 \times 44$ & & $3.84 \mathrm{E}-4$ & 32 & $1.21 \mathrm{E}-3$ & 40 & $2.05 \mathrm{E}-3$ & 46 \\
\hline & Abdominal & $36 \times 44$ & & $1.50 \mathrm{E}-3$ & 32 & $4.28 \mathrm{E}-3$ & 40 & $7.03 E-3$ & 47 \\
\hline & Shoulder & $\begin{array}{l}36 \times 44 \\
28 \times 44\end{array}$ & $\begin{array}{c}\text { Smaller. } \\
\text { beam }\end{array}$ & $\begin{array}{l}6.54 E-2 \\
3.39 E-2\end{array}$ & $\begin{array}{l}33 \\
33\end{array}$ & $\begin{array}{l}1.76 \mathrm{E}-1 \\
1.04 \mathrm{E}-1\end{array}$ & $\begin{array}{l}42 \\
42\end{array}$ & $\begin{array}{l}3.23 \mathrm{E}-1 \\
1.89 \mathrm{E}-1\end{array}$ & $\begin{array}{l}49 \\
49\end{array}$ \\
\hline & Upper G.I. & $36 \times 22$ & & $1.13 \mathrm{E}-3$ & 32 & $3.25 \mathrm{E}-3$ & 41 & $5.43 E-3$ & 47 \\
\hline & Pelvic & $44 \times 36$ & & $8.87 E-4$ & 32 & $2.55 \mathrm{E}-3$ & 41 & $4.43 E-3$ & 47 \\
\hline & Chest & $36 \times 44$ & $\mathrm{PA}$ & $.5 .81 \mathrm{~F}=4$ & 36 & $8.12 \mathrm{E}-3$ & $4 x$ & $2.04 \mathrm{E} 2$ & 55 \\
\hline
\end{tabular}

$a_{w} / \mathrm{s}$ indicates that testes were shielded with a $10 \times 15 \mathrm{~cm}$ piece of loud $0.16 \mathrm{cmi}$ thck. + Indlcates that the detector was located in the primary beam.

PA indicates that the beam was incident posterior-anterior.

${ }^{b} \mathrm{D}$ is the absorbed dose rate per unit tube current. It has units of $\mathrm{mrad} / \mathrm{sec}-\mathrm{mA}$.

${ }^{c}$ The centroid is the centroid of the flucnce vs energy distribution. It has units of keV.

${ }^{d}$ Thyroid shielded by shielding front half of "neck" with lead sheet $8 \mathrm{~cm}$ high and $0.16 \mathrm{~cm}$ thiok. 


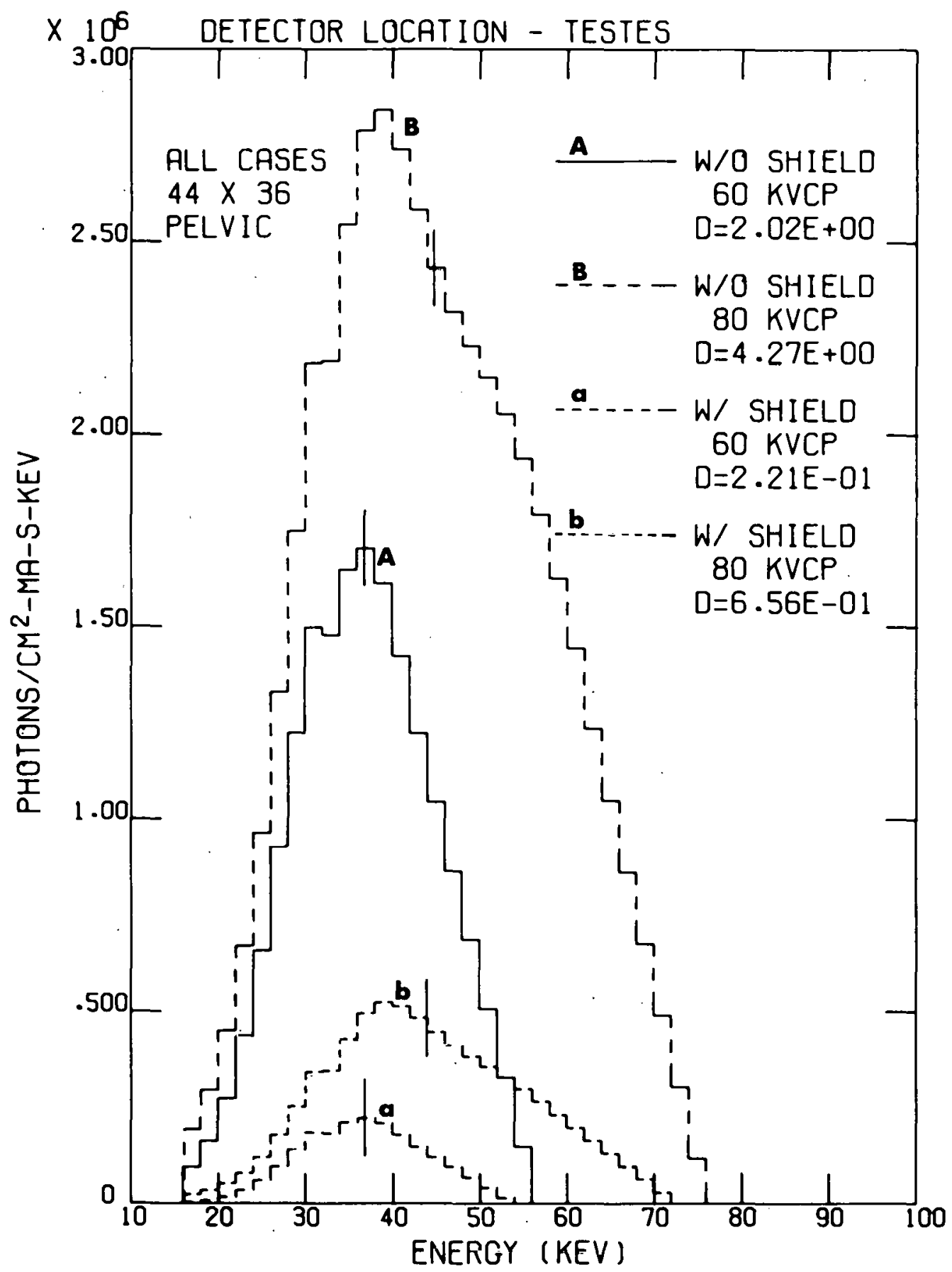

Figure 29. Effect of Gonad Shield on the Spectrum at the Testes -Pelvic Exam (Testes in the Beam). Vertical ticks indicate mean energy. Field size is in $\mathrm{cm}$. $D$ is the absorbed dose per unit current per unit time (mrad/mA-s). 


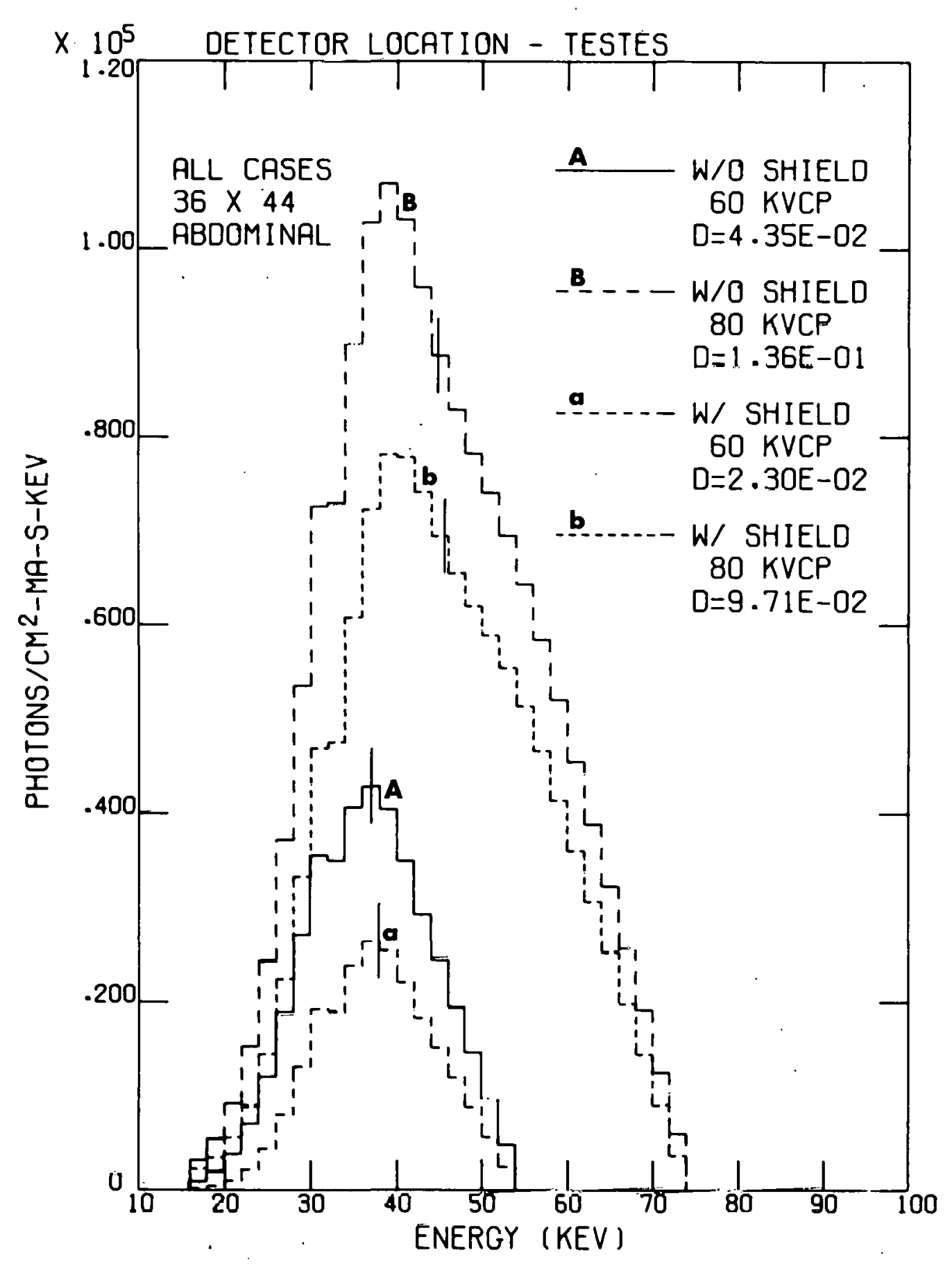

Figure 30. Effect of Gonad Shield on the Spectrum at the Testes -Abdominal Fxam (Testes Near the Beam). Vertical ticks indicate mean energy. Field size is in $\mathrm{cm}$. $D$ is the absorbed dose per unit current per unit time (mrad/mA-s). 


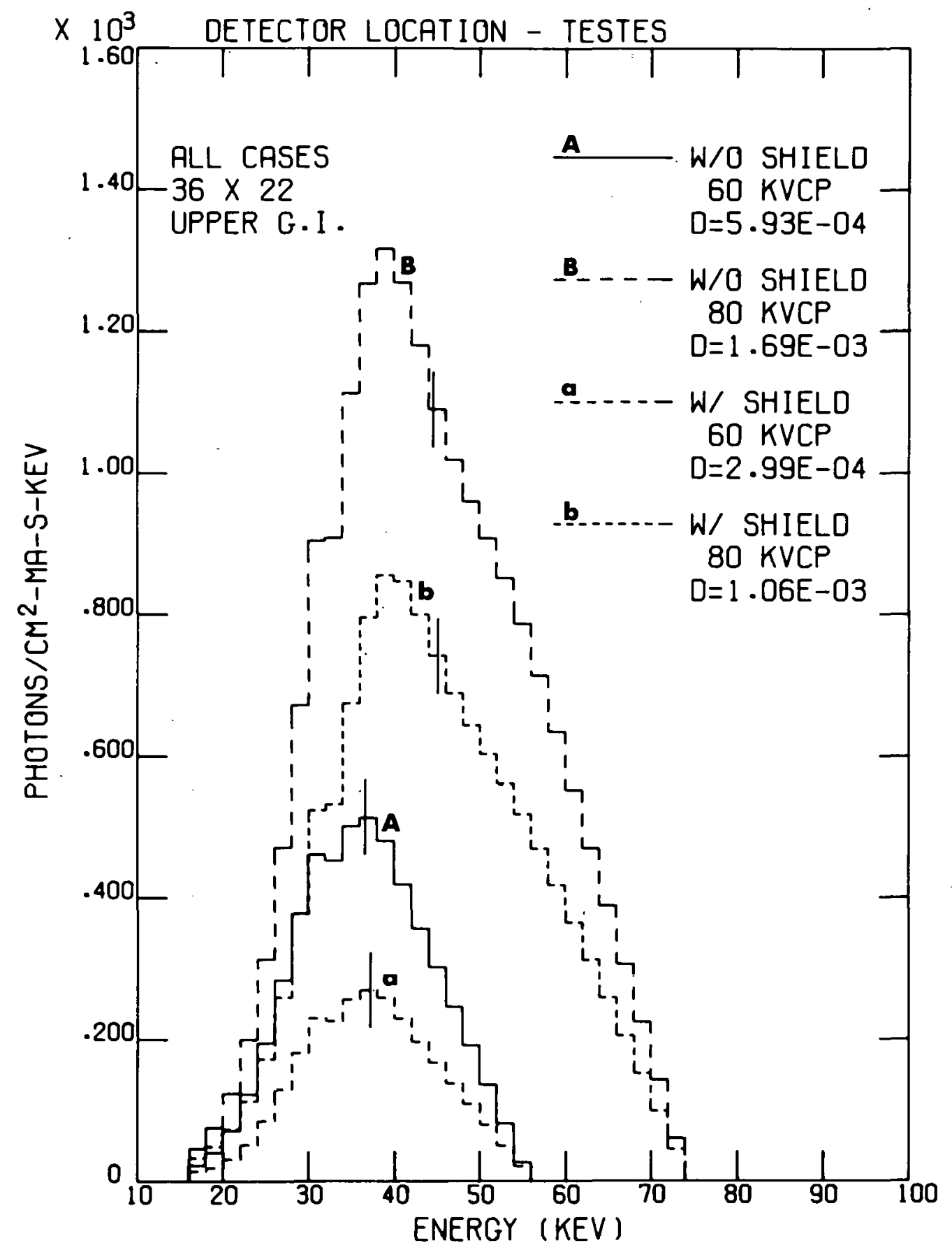

Figure 31. Effect of Gonad Shield on the Spectrum at the Testes -Upper G.I. Exam (Testes Far from the Beam). Vertical ticks indicate mean energy. Field size is in $\mathrm{cm}$. $D$ is the absorbed dose per unit current per unit time (mrad/mA-s). 
dose caused by the shielding in the pelvic exam (in which the testes were in the direct beam). was not surprising. However for the abdominal exam, in which the testes were just out of the beam, and for the upper G.I., in which the testes were farther from the beam, shielding reduced the absorbed dose rates also. These reductions were surprising because they indicate that, for the cases in which the testes are out of the beam, a significant component of the absorbed dose comes from radiation scattered from the collimator. The testes data in Table 7 and in Appendix $A$ show the effect of shielding for all configurations in which a shield was used.

The effect of collimator scattering has been reported previously by Trout and Kelley (Tr65). Because of the manner in which their data were presented, direct comparison between their results and the collimator scatter measured in this study was not feasible.

To ensure that collimator or tube head leaks did not contribute to the spectra measured at the testes, the collimator was enshrouded with additional lead shielding, and the entire collimator and tube housing were checked for radiation leaks. The collimator, with blades closed, and tube housing were wrapped with $x$-ray film and, also, were surveyed by hand with a Geiger counter. No evidence of radiation above background was found.

Shielding also reduced the absorbed dose at the right ovary. For the cases in which the ovaries were not in the direct beam (upper G.I., shoulder, and chest exams), spectra were collected with and without an apron covering the lower part of the torso. The apron, comprised of 
$0.16 \mathrm{~cm}$ thick lead, was placed so that it was just below the lower edge of the beam in all cases. The results are presented graphically in Figures 32-37. Note that the fraction that the absorbed dose is reduced by shielding is largest when the beam is farthest from the right ovary (shoulder exam), but that the amount of the absorbed dose reduction is largest when the beam is closest (upper G.I.).

The effect of shielding on the spectrum and absorbed dose at the thyroid from a shoulder exam was studied. Spectra were collected with and without a shield covering the front half of the neck (lower part of head) of the phantom. Also collected was a spectrum for which the beam was $8 \mathrm{~cm}$ narrower ( $4 \mathrm{~cm}$ on each side). Comparisons of the spectra and absorbed dose rates for these three configurations are given in Figures 38-40. The label "SSFS" stands for "slightly smaller field sizes" and indicates a $28 \times 44 \mathrm{~cm}$ beam rather than a $36 \times 44 \mathrm{~cm}$ beam. Note that shielding the thyroid reduced teh absorbed dose rate was reduced even further when a smaller field size was used instead of a neck shield.

The sparing effect of using a smaller shoulder beam size was studied for the case of the eyes. A comparison of the absorbed dose and spectrum for a $36 \times 44 \mathrm{~cm}$ beam and a $28 \times 44 \mathrm{~cm}$ beam can be made by scrutinizing Figures 99 and 100 in Appendix $A$. In this case the sparing is on the order of a factor of two.

Effect of Depth of Penetration, Scattering Distance and Beam Size

A special series of measurements was conducted to observe changes in the shape of an $x$-ray spectrum as a function of depth of penetration into the phantom. The detector was positioned in the heart region 


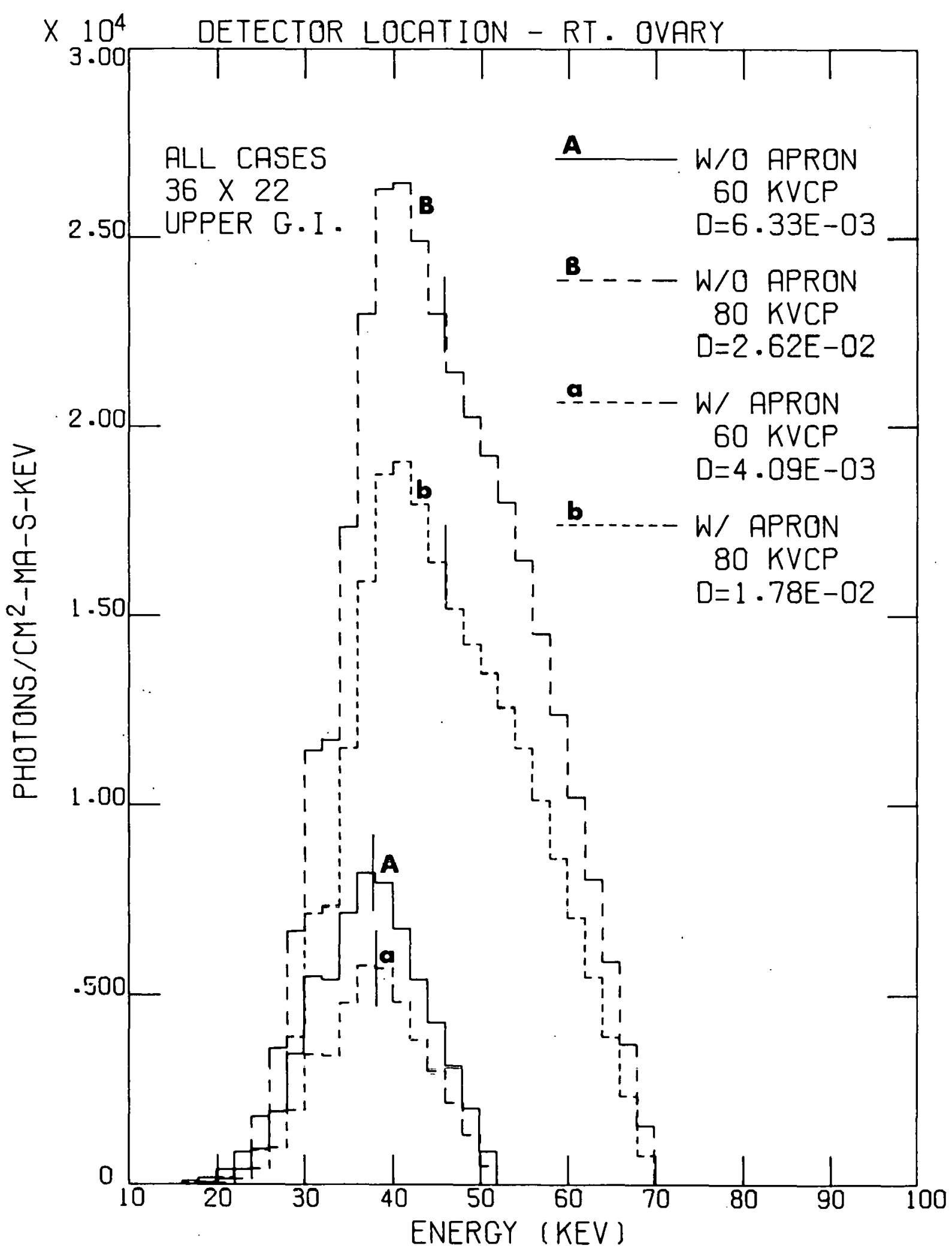

Figure 32. Effect of Shielding with an Apron on the Spectrum at the Right Ovary -- Upper G.I. Exam -- 60 and $80 \mathrm{kVcp}$. Vertical ticks indicate mean energy. Field size is in $\mathrm{cm}$. D is the absorbed dose per unit current per unit time (mrad/mA-s). 


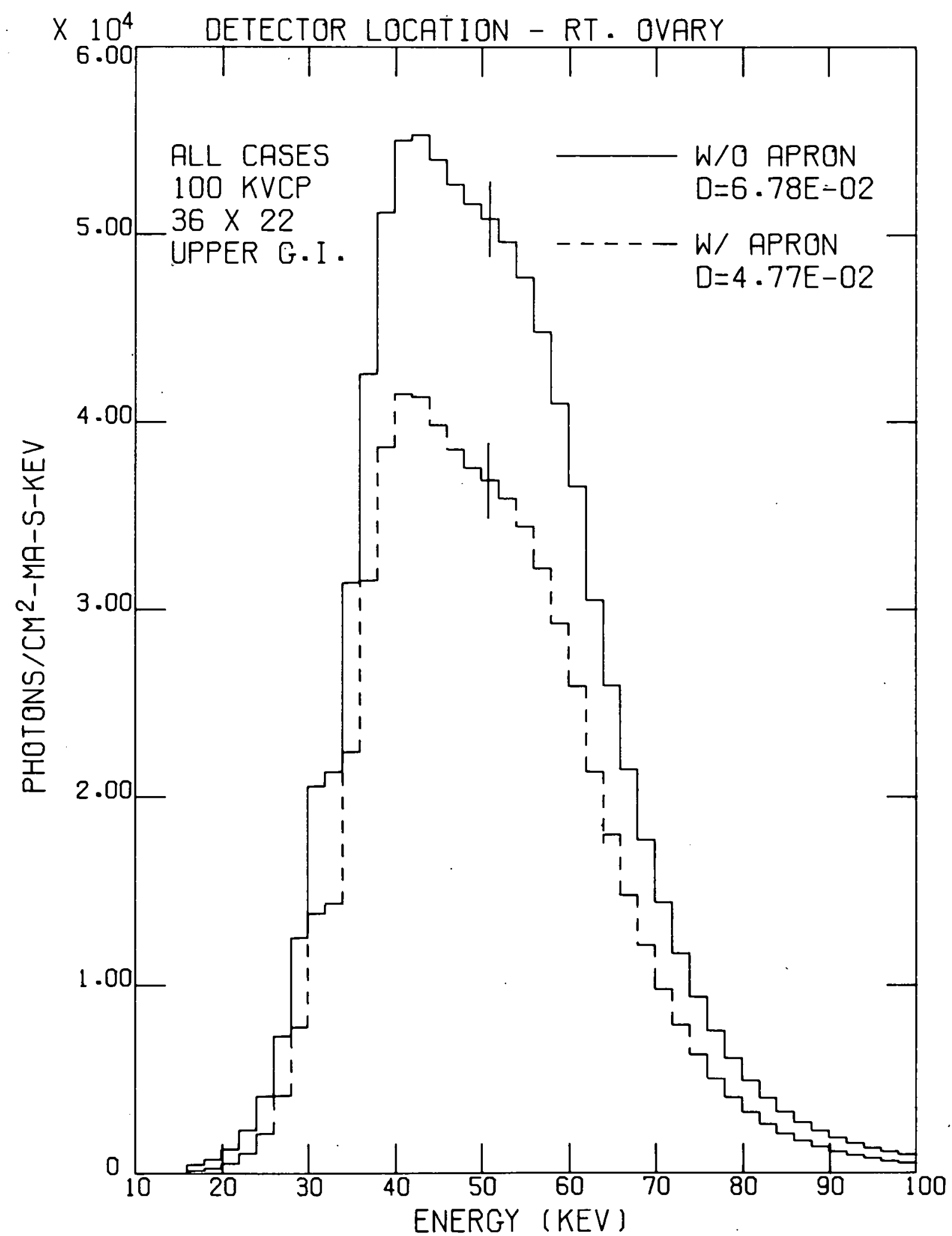

Figure 33. Effect of Shielding with an Apron on the Spectrum at the Right Ovary -- Upper G.I. Exam -- $100 \mathrm{kVcp}$. Vertical ticks indicate mean energy. Field size is in $\mathrm{cm}$. $D$ is the absorbed dose per unit current per unit time (mrad/mA-s). 


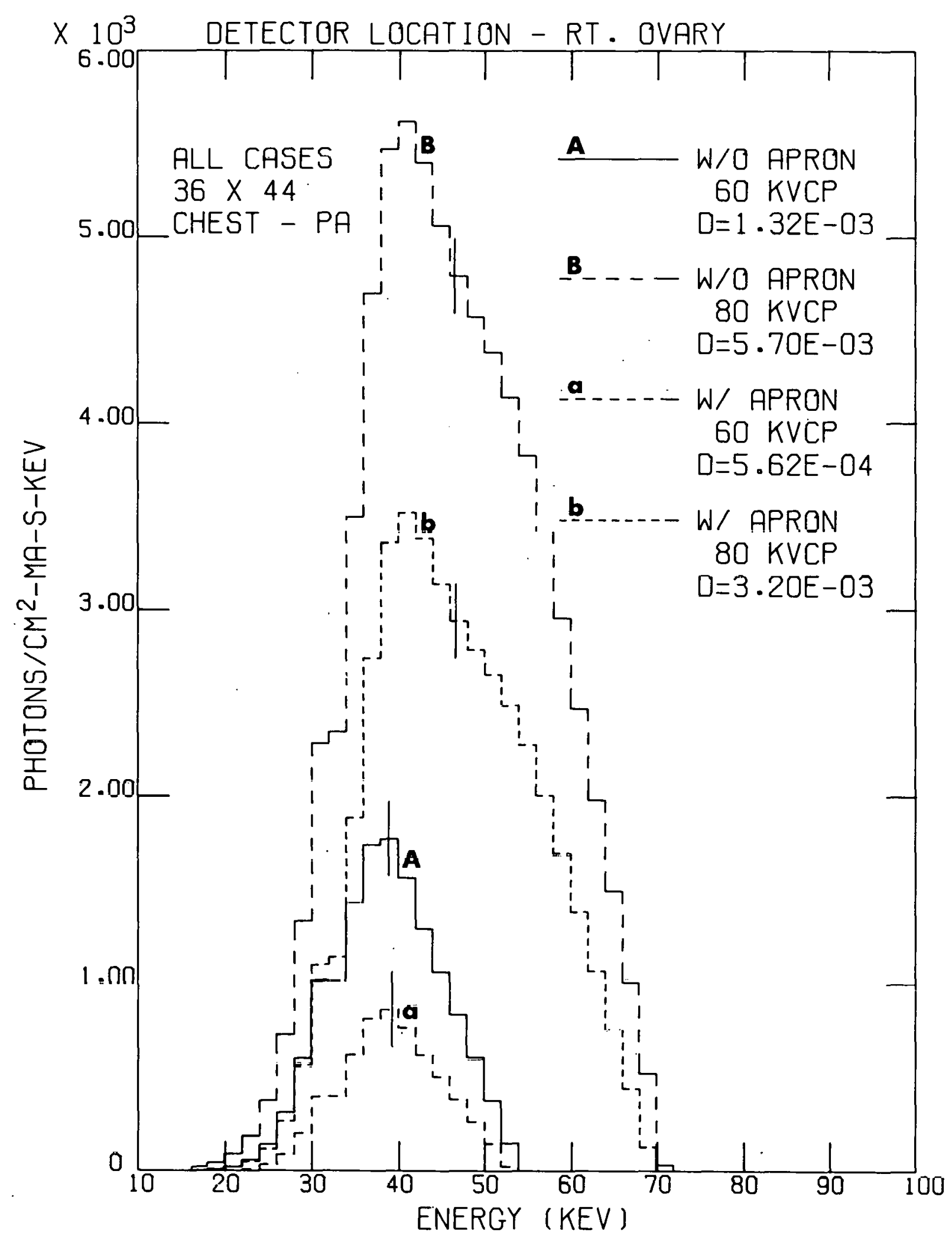

Figure 34. Effect of Shielding with an Apron on the Spectrum at the Right Ovary -- Chest PA Exam -- 60 and $80 \mathrm{kVcp}$. Vertical ticks indicate mean energy. Field size is in $\mathrm{cm}$. $D$ is the absorbed dose per unit current per unit time (mrad/ma-s). 


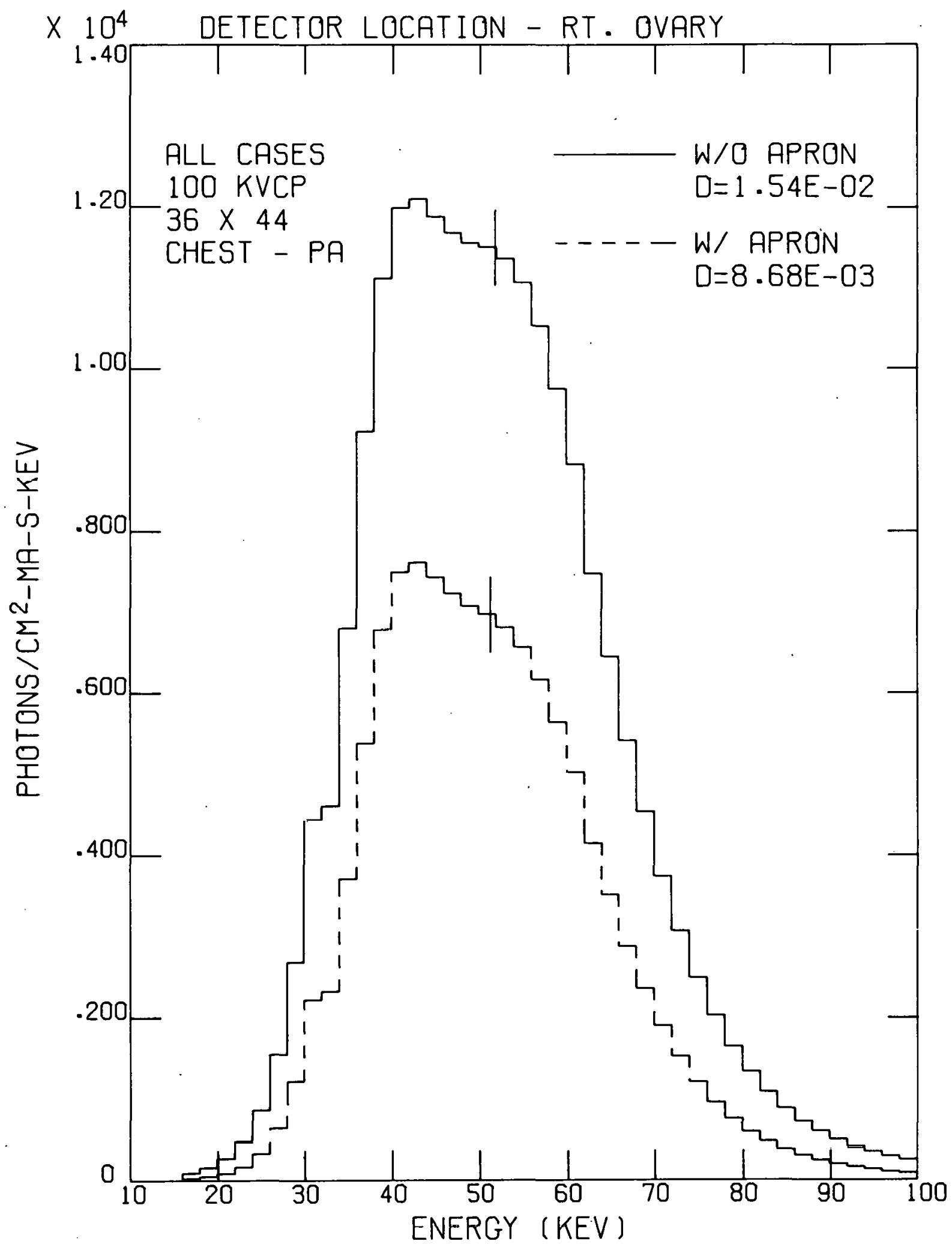

Figure 35. Effect of Shielding with an Apron on the Spectrum at the Right Ovary -- Chest. PA Exam -- 100 kVcp. Vertical ticks indicate mean energy. Fleld size is in $\mathrm{cm}$. $D$ is the absorbed dose per unit current per unit time (mrad/mA-s). 


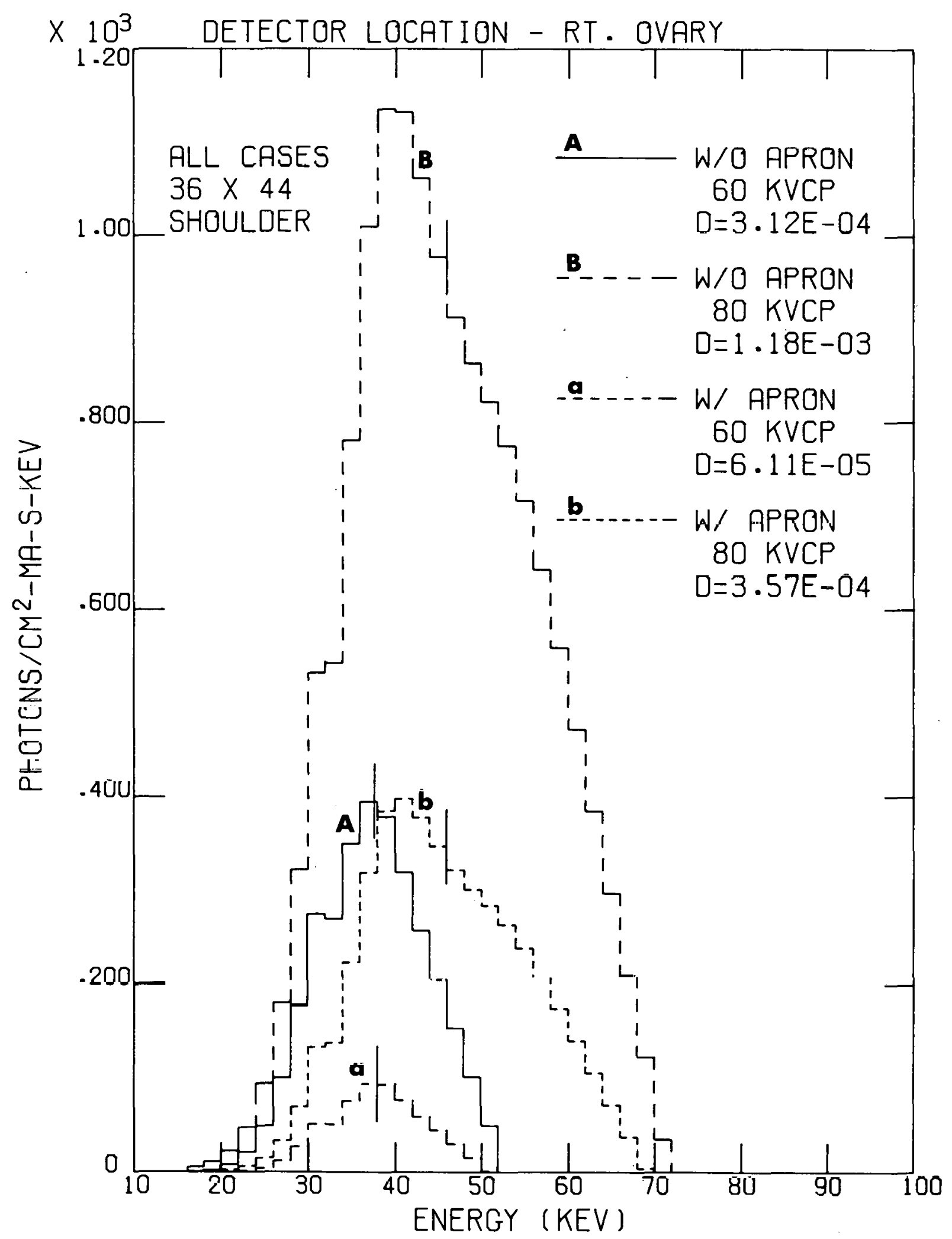

Figure 36. Effect of Shielding with an Apron on the Spectrum at the Right Ovary -- Shoulder Exam -- 60 and $80 \mathrm{kVcp}$. Vertical ticks indicate mean energy. Field size is in $\mathrm{cm}$. D is the absorbed dose per unit current per unit time (mrad/mA-s). 


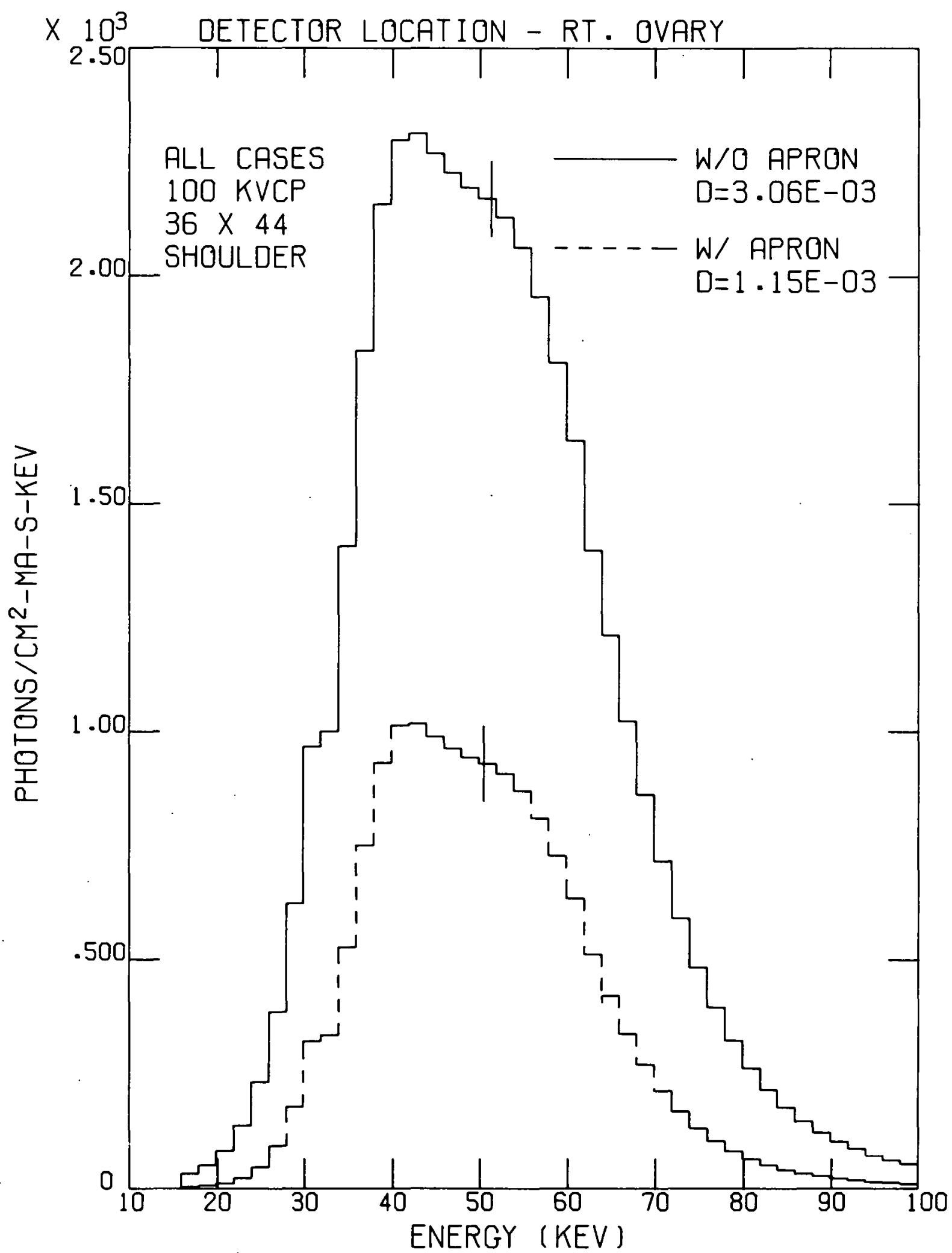

Figure 37. Effect of Shielding with an Apron on the Spectrum at the Right Ovary -- Shoulder Exam -- 100 kVCp. Vertical ticks indicate mean energy. Field size is in $\mathrm{cm}$. D is the absorbed dose per unit current per unit time (mrad/mA-s). 


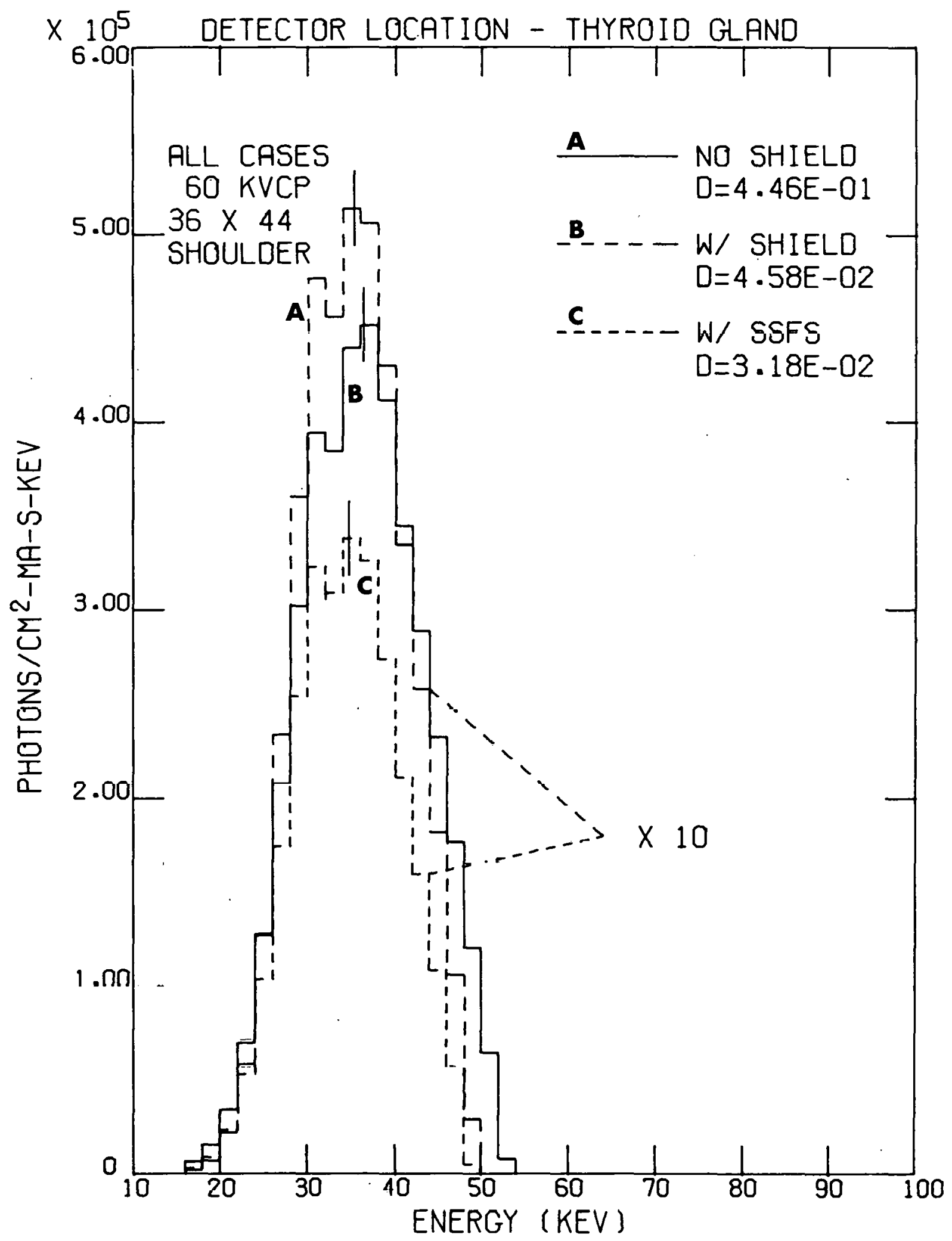

Figure 38. Effect of Shielding and Field Size on the Spectrum at the Thyroid -- Shoulder Exam -- $60 \mathrm{kVcp}$. Vertical ticks indicate mean energy. Field size is in $\mathrm{cm}$. $D$ is the absorbed dose per unit current per unit time (mrad/mA-s). 


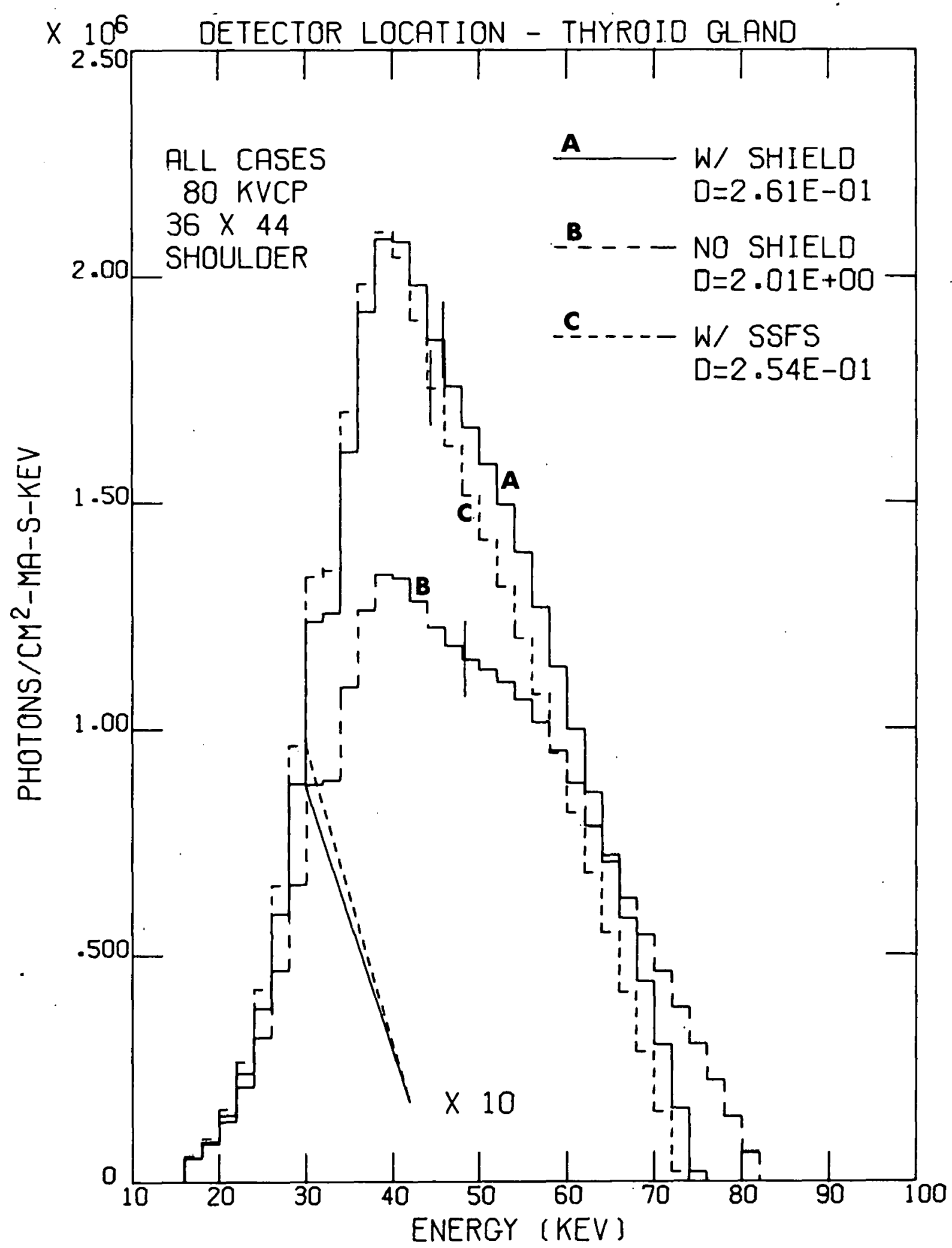

Figure 39. Effect of Shielding and Field Size on the Spectrum at the Thyroid -- Shoulder Exam -- $80 \mathrm{kVcp}$. Vertical ticks indicate mean energy. Field size is in $\mathrm{cm}$. $D$ is the absorbed dose per unit current per unit time (mrad/mA-s). 


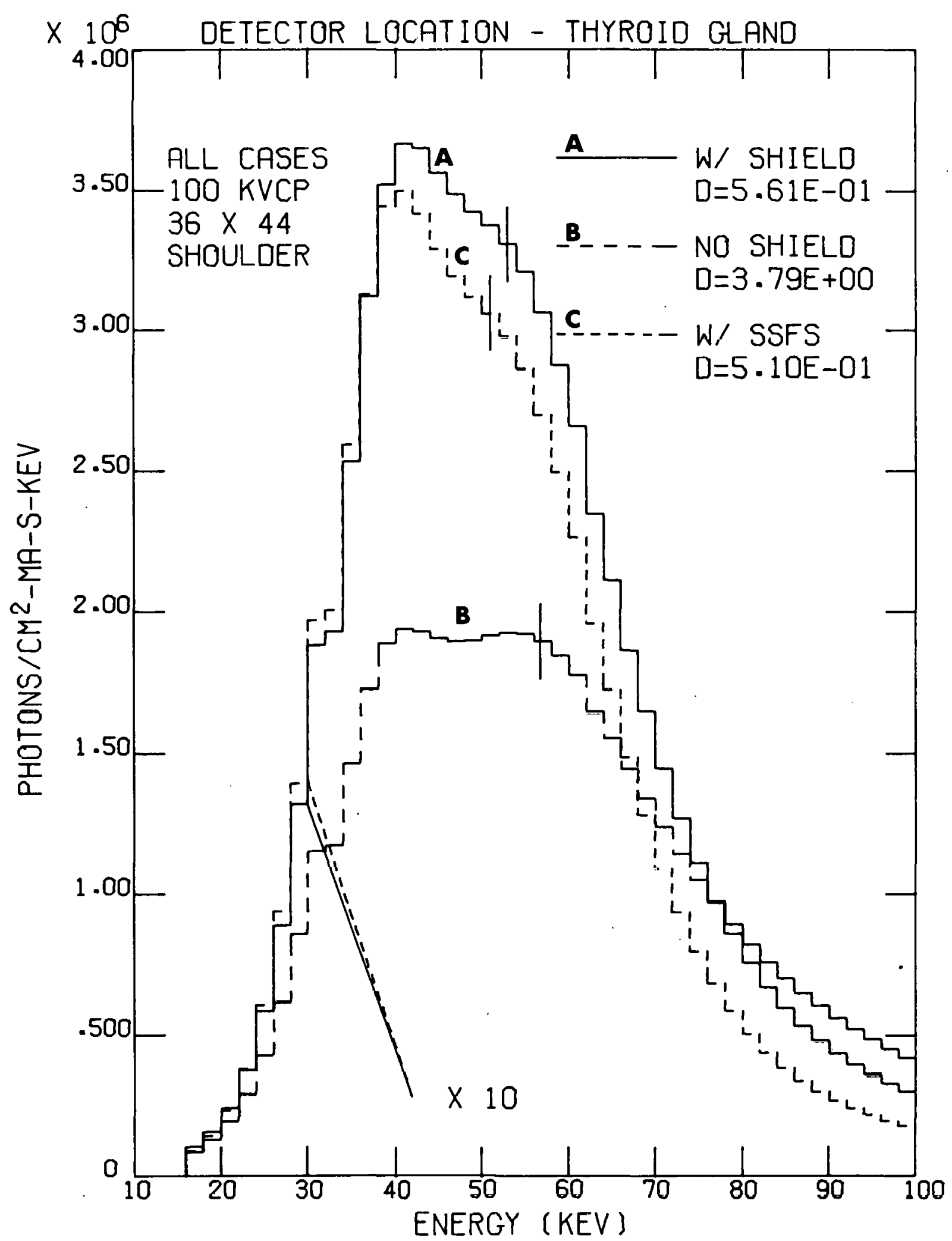

Figure 40. Effect of Shielding and Field Size on the Spectrum at the Thyroid -- Shoulder Exam -- $100 \mathrm{kVcp}$. Vertical ticks indicate mean energy. Field size is in $\mathrm{cm}$. $D$ is the absorbed dose per unit current per unit time (mrad/mA-s). 
location, and a $10 \times 10 \mathrm{~cm}$ beam was centered directly over the active volume of the detector. Spectra were acquired for the detector in the heart region location $(x=0, y=0, z=44)$ and for four other positions each $1 \mathrm{~cm}$ closer to the anterior of the phantom $(x=0, z=44, y=-1$, $-2,-3,-4)$. The results for each generating potential are presented in Figures 41-43. The hardening of the spectrum with depth can be observed by noting the shift of the centroid. The average energy increased $1 \mathrm{keV}$ for the $60 \mathrm{kVcp}$ spectra and $2 \mathrm{keV}$ for the 80 and $100 \mathrm{kVcp}$ spectra over the range of $5 \mathrm{~cm}$ that was studied.

Another series of measurements was conducted to determine the effect of scattering on the spectral distribution. The detector was positioned in the heart region location, and spectra were acquired for six, $10 \times 10$ $\mathrm{cm}$ beams incident on the anterior of the phantom. The six beams were centered at $x=0$ and $z=39,34,29,24,19,14$, respectively. Thus, the projections in the $x-z$ plane of the center of the detector and the center of the $x$-ray fields were separated by $6,11,16,21,26$, and 31 $\mathrm{cm}$. The spectra of scattered radiation for 80 and $100 \mathrm{kVcp}$ cases are presented in Figures 44-47. The detector-field-separation is abbreviated DFS and has units of centimeters. It was observed for both cases that the centroid and the shape of the unfolded spectra varied little over the range of detector-field-separation used.

It was desired to assess the effect of beam size on the spectrum and absorbed dose rate for a combination of beam locations and detector locations. Since the simulated lower spine, full spine, and abdominal exams had the same beam center, data obtained for these cases was amenable to such analysis. The spectra and corresponding absorbed 


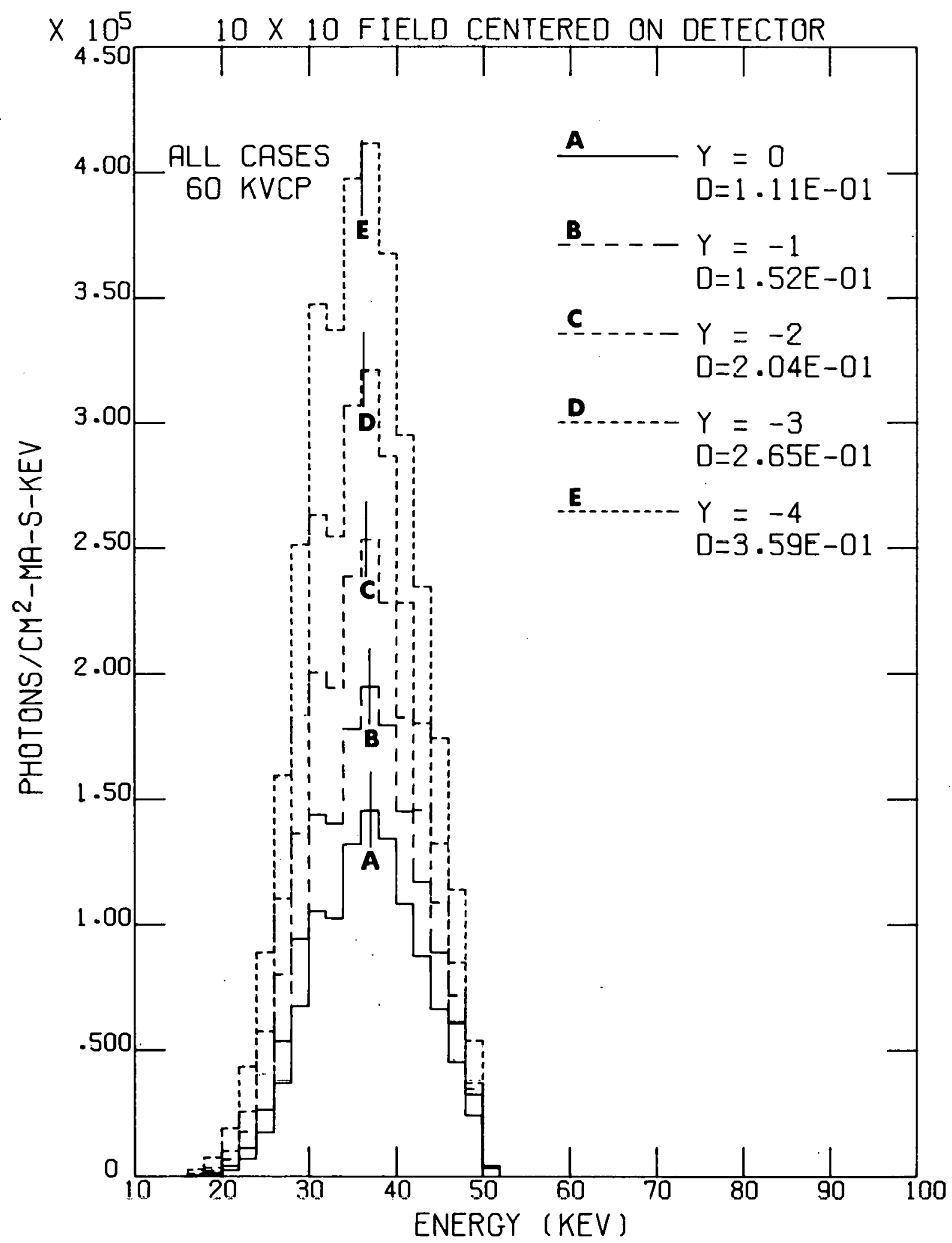

Figure 41. Spectrum as a Function of Depth -- $60 \mathrm{kVcp}$. Vertical ticks indicate mean energy. Field size is in $\mathrm{cm}$. $D$ is the absorbed dose per unit current per unit time (mrad/mA-s). 


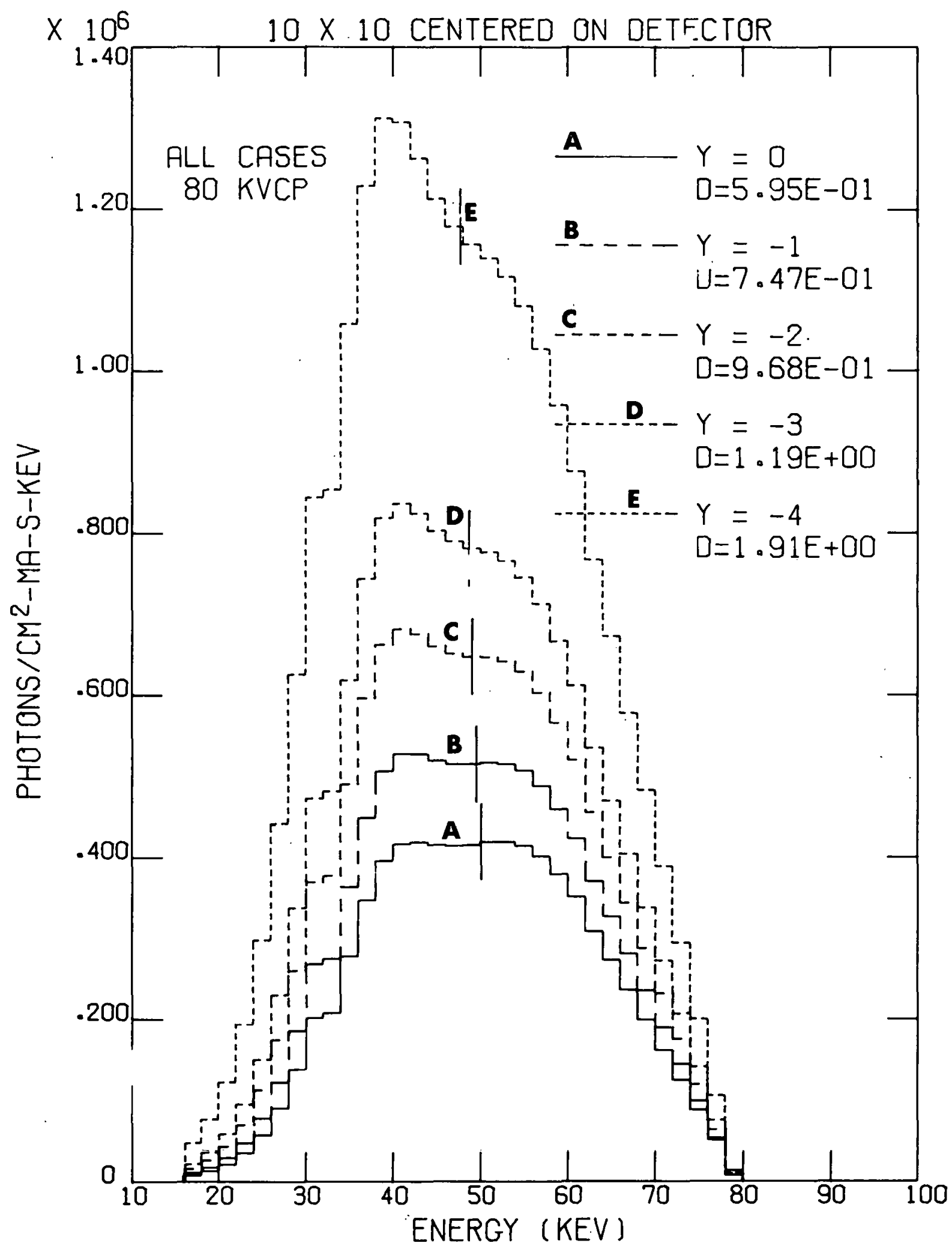

Figure 42. Spectrum as a Function of Depth -- $80 \mathrm{kVcp}$. Vertical ticks indicate mean energy. Field Size is in $\mathrm{cm}$. $D$ is the absorbed dose per unit current per unit time (mrad/mA-s). 
ORNL-DWG 77-13468

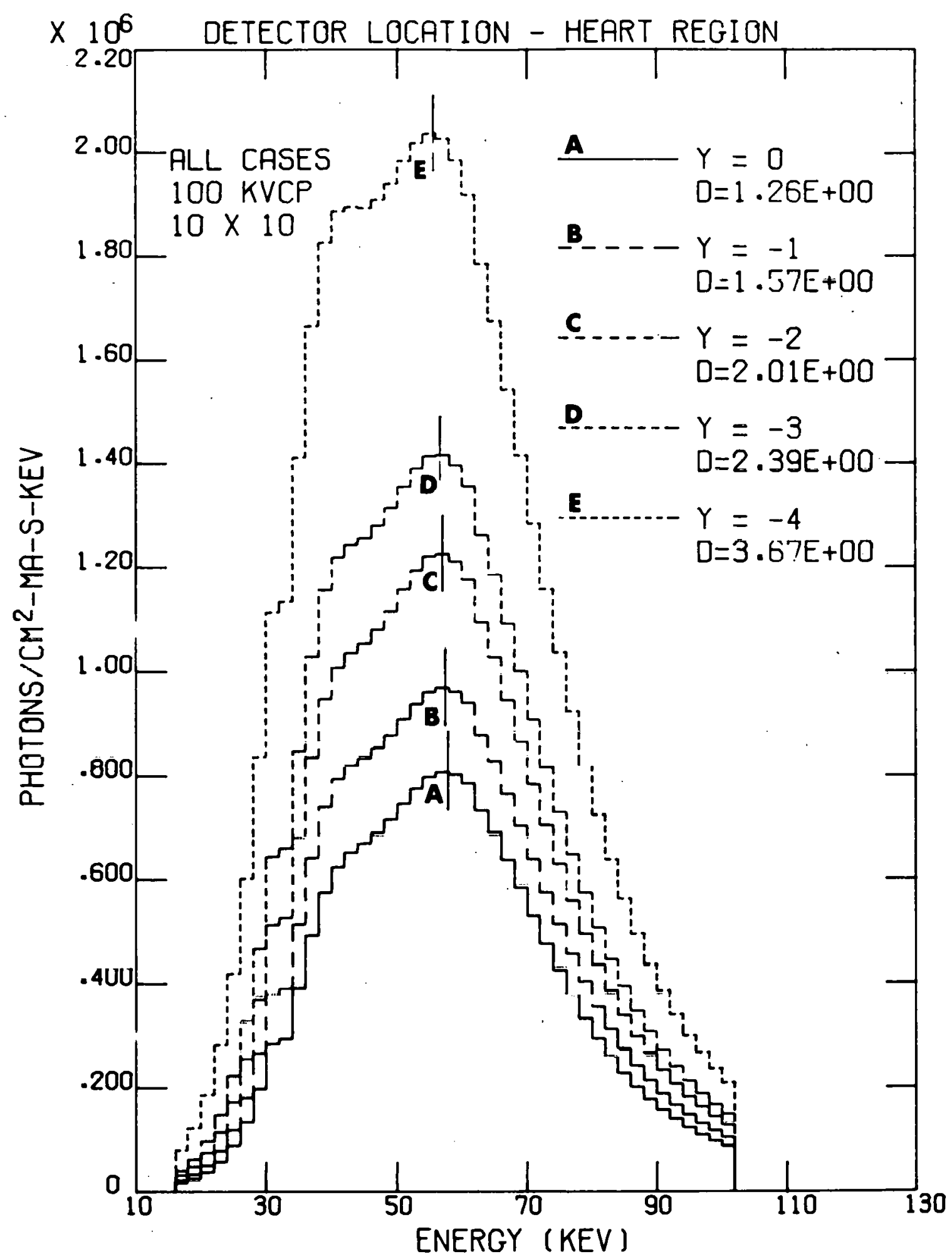

Figure 43. Spectrum as a Function of Depth -- $100 \mathrm{kVcp}$. Vertical ticks indicate mean energy. Field size is in $\mathrm{cm}$. $D$ is the absorbed dose per unit current per unit time (mrad/mA-s). 


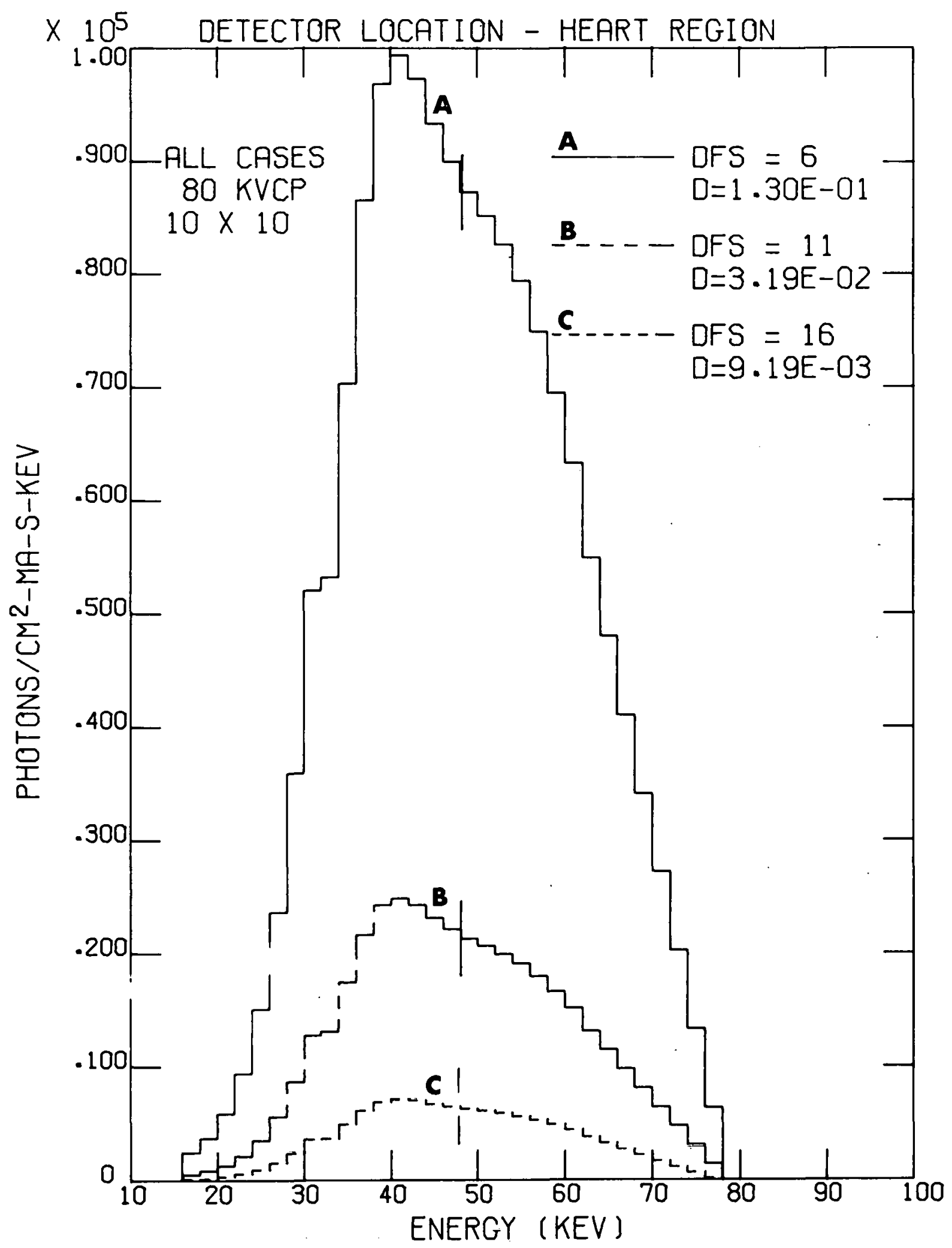

Figure 44. Scattered Spectrum as a Function of Detector-Field-Separation (DFS in Units of Centimeters) -- $80 \mathrm{kVCp}$. Vertical ticks indicate mean energy. Field stze is in $\mathrm{cm}$. $D$ is the absorbed dose per unit current per unit time $(\mathrm{mrad} / \mathrm{mA}-\mathrm{s})$. 


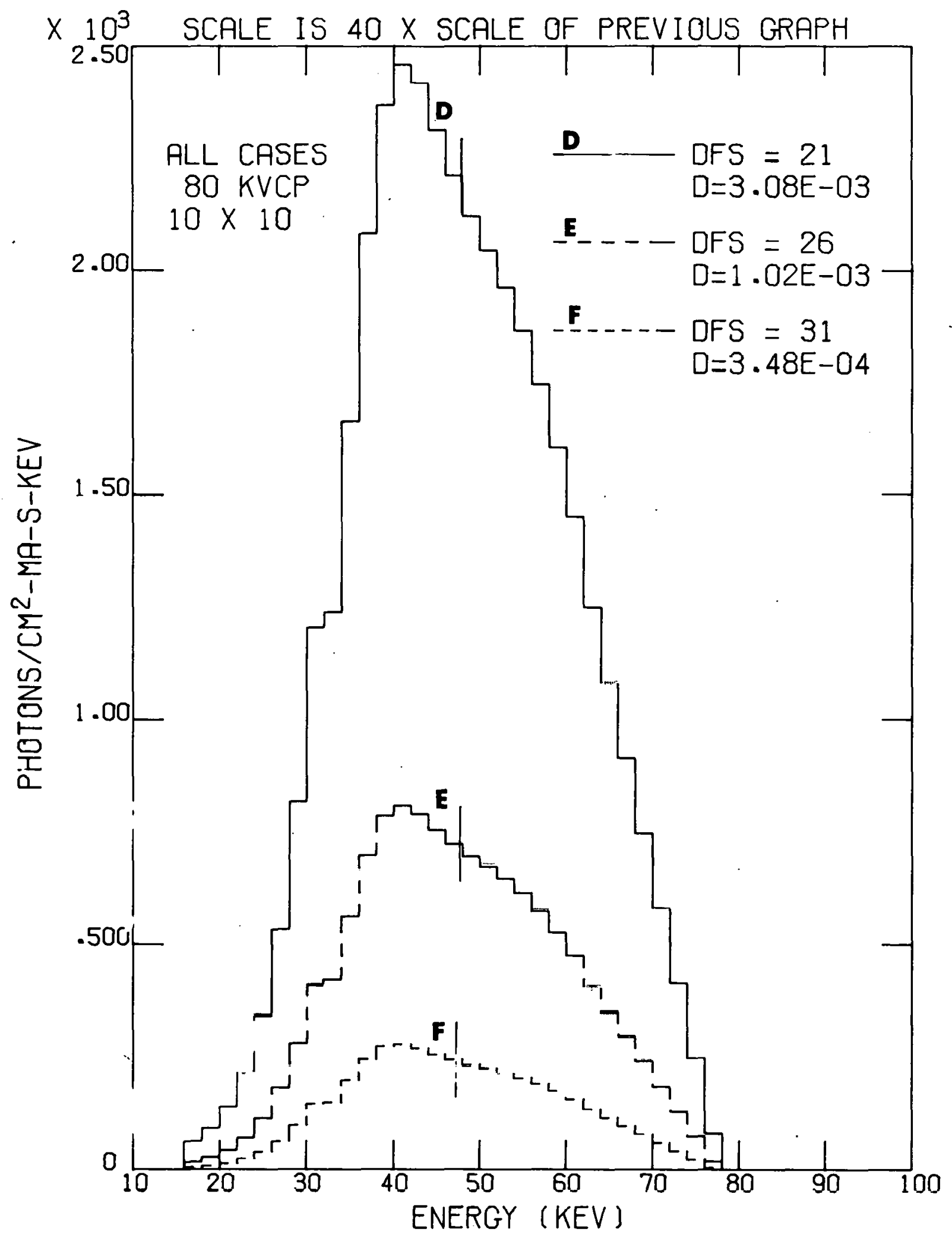

Figure 45. Scattered Spectrum as a Function of Detector-Field-Separation (DFS in Units of Centimeters) -- $80 \mathrm{kVcp}$. Vertical ticks indicate mean energy. Field size is in $\mathrm{cm}$. $D$ is the absorbed dose per unit current per unit time (mrad/mA-s). 
ORNL-DWG 77-13469

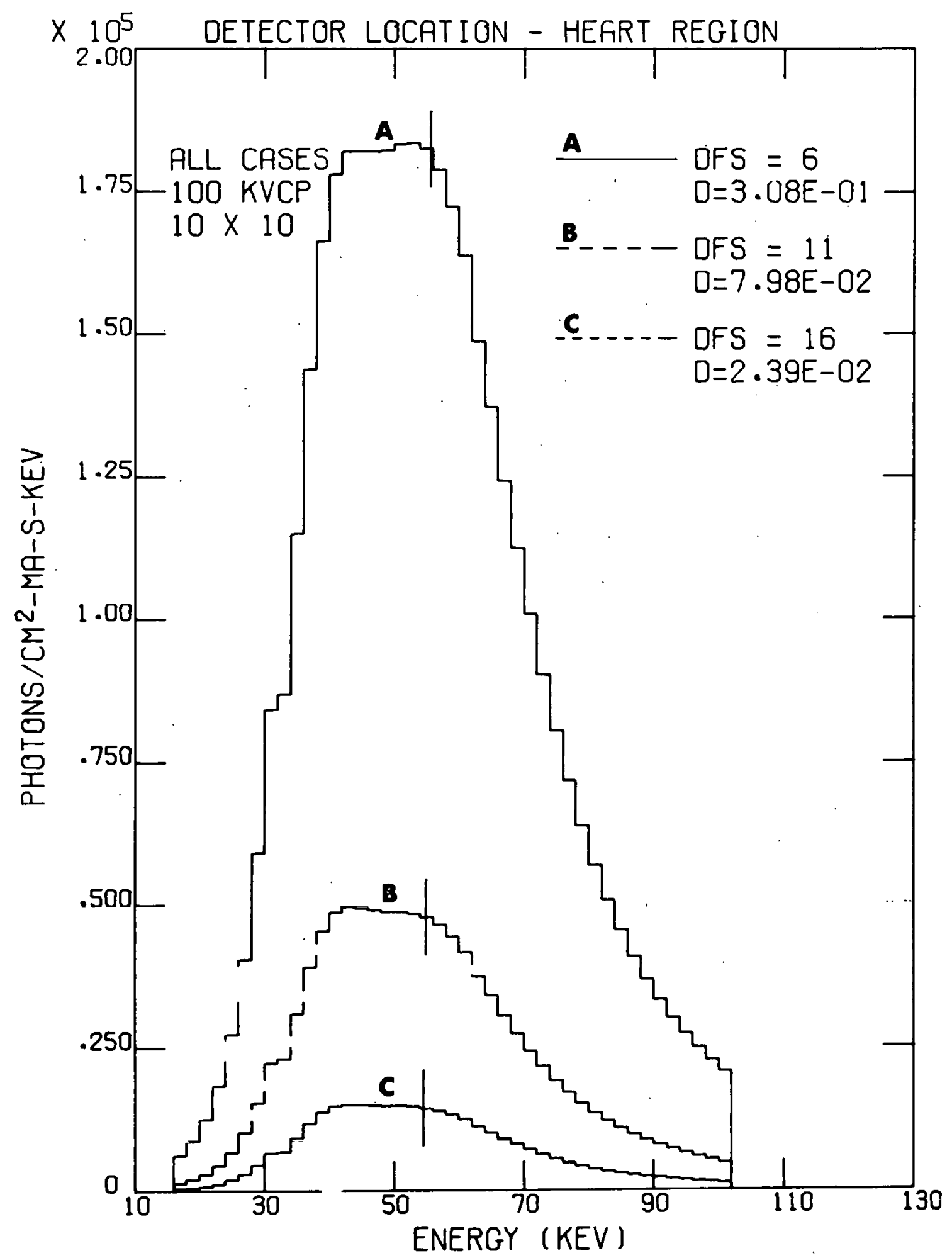

Figure 46. Scattered Spectrum as a Function of Detector-Field-Separation (DFS in Units of Centimeters) -- $100 \mathrm{kVcp}$. Vertical ticks indicate mean energy. Field size is in $\mathrm{cm}$. $D$ is the absorbed dose per unit current per unit time (mrad/mA-s). 
ORNL-DWG 77-13470

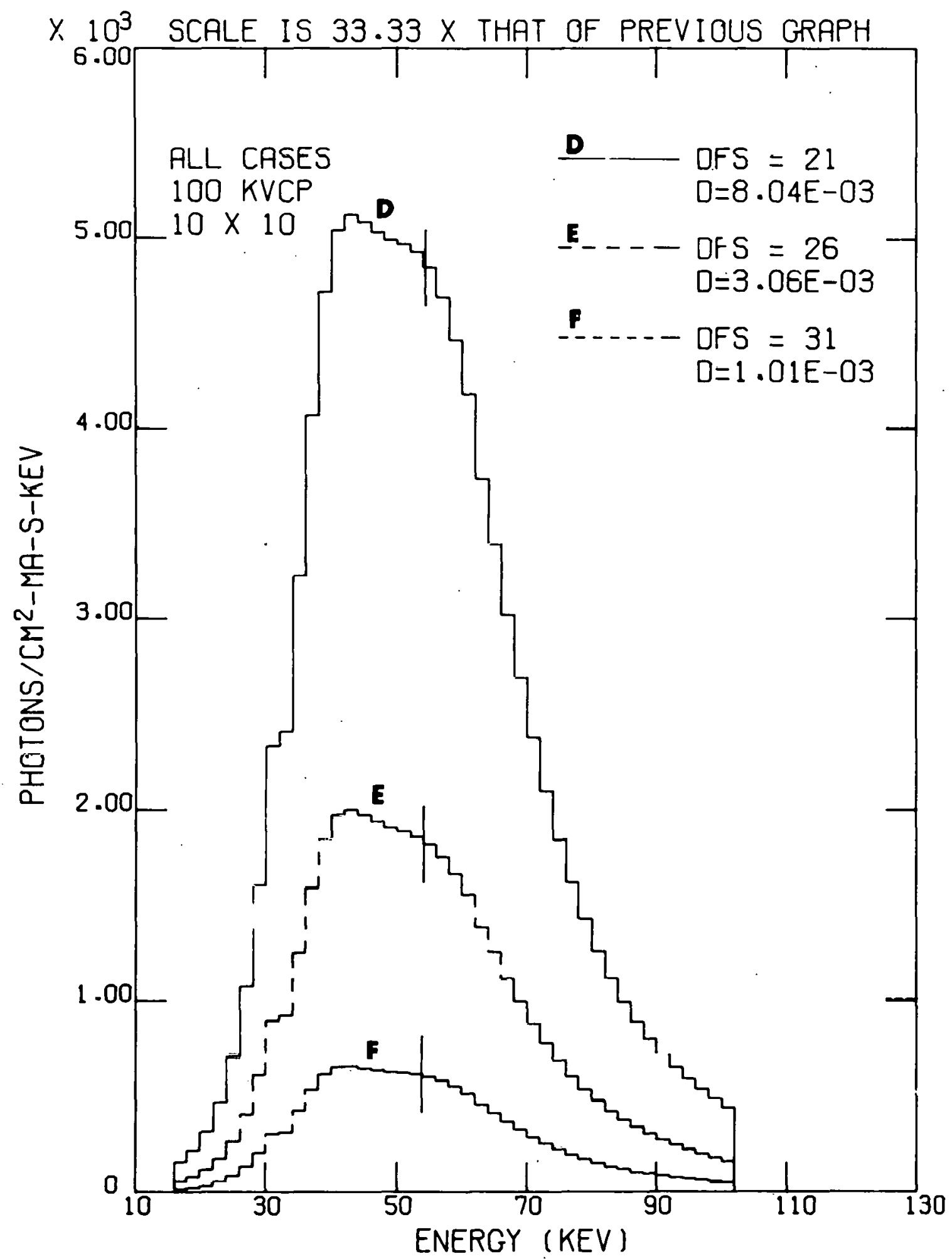

Figure 47. Scattered Spectrum as a Function of Detector-Field-Separation (DFS in Units of Centimeters) -- $100 \mathrm{kVcp}$. Vertical ticks indicate mean energy. Field size is in $\mathrm{cm}$. $D$ is the absorbed dose per unit current per unit time (mrad/mA-s). 
dose rates for these three beam configurations are presented in Appendix $B$ for all combinations of generating potential and detector location (heart region omitted). Except for the cases in which changing the field size affected whether or not the detector was in the direct beam (right ovary or heart locations), the shapes of the spectra varied little as a function of beam size. On the other hand, the absorbed dose rate for any particular combination of generating potential and detector location varied greatly. A quick analysis showed that this variation was not a simple one. In particular, the absorbed dose rate at a particular organ did not vary as a linear function of beam area.

\section{Spectra Inside the Spine}

The spectra measured with the detector in the spine locations were different in three ways from those measured in soft-tissue regions. The "in-bone" spectra had average energies that were generally higher than those in soft-tissue (see Table 8 ). The heart region data and thoracic spine data were selected for comparison since these detector configurations had similar attitudes and locations. The 60,80 , and $100 \mathrm{kVcp}$ spectra for the thoracic spine were harder than the corresponding heart region spectra by about 1, 2, and $3 \mathrm{keV}$, respectively.

Second, the shape of the in-bone spectra was different. In general, the modal energy for any spectrum was below the average energy for that spectrum. However, the difference between the mean energy and the modal energy was usually smaller for the in-bone spectra than for the softtissue spectra. See Figures 67-67 and 89-95 in Appendix A. Third, the magnitude (modal energy height, total fluence, or absorbed dose) was a 
more sensitive function of generating potential for spectra acquired in the spine than for spectra acquired in other regions. Note that the 60 $k V c p$ spectra for spine locations generally were graphed with a scaling factor of 10 in order to make them comparable with their respective 80 and $100 \mathrm{kVcp}$ spectra. For soft-tissue region spectra, such scaling often was not necessary.

\section{Error Analysis}

The major sources of error in this study were judged to have arisen from the non-uniformity of the response of the detector as a function of angle of incidence, the inaccuracies in the response functions used to unfold the pulse height spectra, the low resolution of the spectrometry system in general, and the dissimilarity between the spectral output of the $x$-ray machine and spectra more representative of diagnostic radiology. It is important that the effects and limitations imposed by these sources of error be kept in mind when the data gathered in this study are interpreted.

As is presented in Figure 12, the response of the detector was a function of both energy and declination of a beam of incident $x$-rays . Since the angular distribution of an $x$-ray field within the phantom was unknown in general, it was not possible to correct for this non-uniformity of response. As was discussed previously, the detector was oriented to minimize such uncertainty. If it is presumed that the majority of the photons striking the detector were incident with a declination between $\pm 45^{\circ}$, the uncertainty in energy would be 1 imited. For $50 \mathrm{keV}$ photons incident within the described solid angel, the variation in the mean of the observed pulse height distribution would be approximately $5 \%$. 
Therefore, it is judged that the uncertainty in the centroids of the unfolded spectra (all of which are near $50 \mathrm{keV}$ ) is $\pm 2 \mathrm{keV}$.

The response functions used to unfold the pulse height spectra were formulated by combining data from Monte Carlo calculations and measured characteristics of the detector. The Monte Carlo calculations were performed for an exactly spherical volume of $\mathrm{NaI}$, whereas the detector itself was slightly oblate. Obviously, the probability of Compton scattered photons and fluorescent $x$ rays escaping the detector is dependent on the shape of the detector. Other inaccuracies in the response functions were deduced by examination of the unfolded spectra. All spectra suffered from the "rounding of the corners" caused by the inherent poor resolution of the system. Also each unfolded spectrum had some inaccuracy near $32 \mathrm{keV}$, the iodine K-edge. Initial calibration of the detector showed a change in linearity at this energy. Apparently this change was not represented completely properly in the response functions because the unfolded spectra displayed an artificial "fine structure" at $32 \mathrm{keV}$. It was not known how to assess the overall effect of the inaccuracies described above. It is suggested that the performance of the unfolding procedure be gauged by the examples of the unfolding of known spectra given previously (Figures 17 and 18).

In order to have an $x$-ray machine with the wide dynamic range and other characteristics essential to this project, it was necessary to accept output spectra that were significantly different than those typical of medical diagnostic radiology. The x-ray machine's output spectra were particularly dissimilar in the region near $37 \mathrm{keV}$, 
the barium K-edge (see Figure 10). Measured spectra which heavily depend on the shape of the output spectra in this region should be viewed as approximations of those spectra which would be present in actual diagnostic examinations. For example, the spectrum at the testes due to a pelvic exam should be considered very approximate since the beam of $x$-rays has penetrated only a few centimeters of tissue. However, as a beam continues to penetrate the phantom, the photons with energy near $37 \mathrm{keV}$ would be attenuated and would be replaced by degraded photons which originally had a higher energy. Thus spectra at locations deep in the phantom or outside the primary beam would not be as strongly influenced by the low energy components of the output spectra and should be more representative of those that are present in an actual diagnostic exam. How well the spectra of a particular x-ray machine resembles the spectra of the $x$-ray machine used in this study may influence the applicability of the data presented in this study.

Other sources of error include the statistical errors inherent in $x$-ray detection, short-term and long-term fluctuations in the electronic equipment including the high voltage stabilization circuitry of the $x$-ray machine, the inaccuracies in the phantom and its tissue-equivalent materials, and the positioning of the probe and the $x$-ray beams. It is estimated that the probe was positioned within the phantom with an accuracy of $\pm 0.3 \mathrm{~cm}$ and that the beam was aligned with a similar uncertainty. These uncertainties are well within the variation that would be typical of actual diagnostic examinations. The uncertainties due to electronic equipment and the phantom are judged to be of much lesser significance than the errors discussed in the preceding paragraphs. 
A set of ionization chamber measurements were made to provide data with which to estimate the accuracy of the absorbed dose rates calculated from the measured spectra. For reasons of convenience and feasibility, measurements were made in the testes regions with the $3 \mathrm{~cm}^{3}$ probe of the Radocon III. The measured absorbed dose rates at the testes for three beam configurations and two generating potentials are presented in Table 10 along with the absorbed dose rates determined from the corresponding spectral mesurements. The absorbed dose rates determined from the spectrum measurements vary from $16 \%$ lower to $6 \%$ higher than the absorbed dose rates measured with the ionization chamber. However, the difference between the two types of measurements did not appear to be random. Excepting one case, the absorbed dose rates determined from the spectrum measurements were smaller than the results indicated by the ionization chamber. Two explanations for this difference are given. The absorbed dose determined with the spectrometer should be lower than the actual absorbed dose because the truncation and extrapolation processes used to adjust the unfolded spectrum decrease the total fluence slightly. Second, the size of the ionization chamber influenced its response compared with the spectrometer. The radius of the ionization chamber was $1.3 \mathrm{~mm}$ larger than the radius of the beryllium cap of the spectrometer. Hence, the ionization chamber displaced more tissue fluid than the spectrometer, and, under similar conditions, more $x$ rays would reach the ionization chamber than would reach the spectrometer because of absence of the displaced tissue fluid. Nevertheless, the purpose of the ionization chamber measurements was to check the general validity of the method of determining the absorbed 
TABLE 10. Comparison of Ionization Chamber Measurements with the Absorbed Dose Rate Jetermined from the Measured Spectrum at the Testes

\begin{tabular}{|c|c|c|c|c|}
\hline $\begin{array}{l}\text { Generating } \\
\text { Potential } \\
(k \vee c p)\end{array}$ & Beam Type & $\begin{array}{l}\text { Absorjed Dose Rate } \\
\text { Measured with } \\
\text { Ionization Chamber } \\
\text { (rarad.mA-sec) }\end{array}$ & $\begin{array}{l}\text { Absorbed Dose Rate } \\
\text { Calculated from } \\
\text { Measured Spectrum } \\
\text { (mrad/mA-sec) }\end{array}$ & Percent Difference ${ }^{b}$ \\
\hline \multirow[t]{3}{*}{80} & Ful: Ssine & $2.35 \times 10^{-\tilde{c}} \pm 7 \%^{\mathrm{a}}$ & $1.97 \times 10^{-2}$ & $-16 \%$ \\
\hline & Abdominal & $5.21=10^{-2} \pm 7 \%$ & $4.35 \times 10^{-2}$ & $-16 \%$ \\
\hline & Pelvic & $2.39 \pm 7 \%$ & 2.02 & $-16 \%$ \\
\hline \multirow[t]{3}{*}{100} & Full Spine & $0.121 \pm 7 \%$ & 0.117 & $-3 \%$ \\
\hline & Abdaminai & $0.283 \pm 7 \%$ & 0.299 & $6 \%$ \\
\hline & Pelvic & $8.50 \pm 7 \%$ & 7.70 & $-9 \%$ \\
\hline
\end{tabular}

ancertainty includes $\pm 4 \%$ for angular response of probe and $\pm 3 \%$ for calibration uncertainty. It does not include any uncertainty due to geometrical considerations.

$b_{\text {Spectrometer Measuremert - Ionization Chamber Measurement }}$

Ionization Chamber Measuremen: 
dose from the measured spectra. In this respect, the agreement between the absorbed doses was adequate.

\section{Comparison with BRH Calculations}

As was discussed in Chapter III, the U. S. Bureau of Radiological Health published the results of a Monte Carlo study of absorbed doses tô selected organs for common projections in diagnostic radiology (Ro76b). In this study a divergent beam of $x$-rays was simulated with a combination of $4 \times 4 \mathrm{~cm}$ parallel beams. Further instead of making calculations for continuous spectra typical of diagnostic radiology, the absorbed doses were calculated as a linear combination of 5 monoenergetic beams. The calculations were made using the mathematical model on which the MR. ADAM phantom is based. Absorbed doses were calculated for the testes, ovaries, thyroid, active bone marrow region, and uterus. Typical exposure configurations were assumed and the results are presented as a function of half-value-layer (HVL).

The results for the testes, ovaries, and thyroid for the chest PA, pelvic, and abdominal examinations as measured in this study are compared in Table 11 with the results of the BRH calculations most similar. In the calculated chest PA case, the focal-spot-to-film distance employed was $183 \mathrm{~cm}$; in all measurements a distance of $120 \mathrm{~cm}$ was used. The hip exam in the BRH calculations had a configuration best resembling the pelvic exam simulated in the measurements. The abdominal exams in both studies were essentially the same. Due to statistical uncertainties, the calculated absorbed dose in some cases was presented as a small but unestimated number (i.e., $0.01 \mathrm{mrad}$ ). 
TABLE 11. Comparison of Measured Absorbed Dose with BRH Calculations

\begin{tabular}{|c|c|c|c|c|c|c|c|c|c|c|}
\hline \multirow{2}{*}{ Beam Type } & \multirow{2}{*}{$\begin{array}{l}\text { Organ of Interest } \\
\text { Generating Potential :kVcp) } \\
\text { HVL ( } \quad \text { mAl) }\end{array}$} & \multicolumn{3}{|c|}{ Testes } & \multicolumn{3}{|c|}{ Ovaries } & \multicolumn{3}{|c|}{ Thyroid } \\
\hline & & $\begin{array}{r}60 \\
1.8\end{array}$ & $\begin{array}{r}80 \\
2.6\end{array}$ & $\begin{array}{l}100 \\
3.3\end{array}$ & $\begin{array}{r}60 \\
1.8\end{array}$ & $\begin{array}{r}80 \\
2.6\end{array}$ & $\begin{array}{l}100 \\
3.3\end{array}$ & $\begin{array}{r}60 \\
1.8\end{array}$ & $\begin{array}{r}80 \\
2.6\end{array}$ & $\begin{array}{l}100 \\
3.3\end{array}$ \\
\hline \multirow[t]{3}{*}{ Chest PA } & $\begin{array}{l}\text { Calculated Absorbed Dose } e^{a} \\
\text { Reference Uncertainties }\end{array}$ & $<0.01$ & $\begin{array}{l}<0.01 \\
i](0 \%, N C)\end{array}$ & $<0.01$ & 0.4 & $(5: 3 \%, N C)$ & 2.4 & $15 \quad 19$ & $9 \%, \quad 50 \%)$ & 48 \\
\hline & Measured Absorbed Dosec & 0.033 & 0.065 & 0.15 & 0.15 & 0.89 & 1.9 & 5.2 & 25 & 43 \\
\hline & Percent Difference ${ }^{C}$ & * & * & $\star$ & $-18 \%$ & $-19 \%$ & $-21 \%$ & $-65 \%$ & $-22 \%$ & $-10 \%$ \\
\hline \multirow[t]{3}{*}{ Pelvic } & $\begin{array}{l}\text { Calculated Absorbed Dosea,t } \\
\text { Reference Uncertaintiese }\end{array}$ & 727 & $(1 \%, 1 \%)$ & 974 & 122 & $(6 \%, 57 \%)$ & 271 & $<0.01$ & $\begin{array}{c}<0.01 \\
(N C, N C)\end{array}$ & $<0.01$ \\
\hline & Measured Absorbed Dosea & 652 & 667 & 939 & 48 & 101 & 177 & 0.054 & 0.14 & 0.23 \\
\hline & Percent Differenc $c c$ & $-10 \%$ & $-25 \%$ & $-4 \%$ & $-61 \%$ & $-51 \%$ & $-35 \%$ & * & $\star$ & $\star$ \\
\hline \multirow[t]{3}{*}{ Abdominal } & $\begin{array}{l}\text { Calculated Absorb } 2 d \text { Dcsea } \\
\text { Refererice Uncertaintiese }\end{array}$ & 10 & $(4 \%, 38 \%)$ & 26 & 128 & $\begin{array}{c}220 \\
(5 \%, 14 \%)\end{array}$ & 291 & $<0.01$ & $\begin{array}{l}<0.01 \\
(\mathrm{NC}, \mathrm{NC})\end{array}$ & $<0.01$ \\
\hline & Measured Absorbed Doset: & 14 & 21 & 36 & 112 & 156 & 365 & 0.16 & 0.45 & 0.81 \\
\hline & Percent [iifference ${ }^{c}$ & $40 \%$ & $17 \%$ & $38 \%$ & $-13 \%$ & $-29 \%$ & $-25 \%$ & * & * & * \\
\hline
\end{tabular}

*Undetermined.

${ }^{a}$ Average absorbed dose (mrad) For Exposure at surface of the phantom of $1000 \mathrm{mR}$ as calculated (Ro76).

${ }^{b}$ Absorbed dose determined from spectra measurements made at the center of the organ of interest. Absorbed dose is normalized to $1000 \mathrm{mF}$. insiden: excosume.

$c_{\text {measured-calculated }}$

\section{calculated}

${ }_{\text {Absorbed dose for both hips expos,re configuratior. }}$

e (Coefficient of variation for $4 \times 4 \mathrm{~cm}$ beam closest to the organ of interest, coefficient of variation for $4 \times 4$ beam at center of field). NC meais no calculated contribution for this $4 \times 4$ beam. 
Since the BRH publication presented no estimated error with each calculation, Table 11 contains a pair of numbers called reference uncertainties. These numbers were abstracted from the results of the monoenergetic calculations (Ro76a). They are the coefficients of variation for two of the $55 \mathrm{keV} 4 \times 4 \mathrm{~cm}$ beams used in the BRH study. the first of the pair is the coefficient of variation for the $4 \times 4 \mathrm{~cm}$ (contained in the projection) which passes through or comes closest to the organ of interest. The second of the pair is the coefficient of variation for the $4 \times 4 \mathrm{~cm}$ at the center of the projection. The label "NC" indicates that the respective $4 \times 4 \mathrm{~cm}$ beam did not contribute to the absorbed dose at the organ of interest, and, therefore, the coefficient of variation is undetermined. The reference uncertainties presented have no direct relation to statistical uncertainty in the result of the Monte Carlo study. However, since the calculated absorbed dose estimates were based on a linear combination of the results of a set of $4 \times 4 \mathrm{~cm}$ beams at various monoenergies, the reference uncertainties may give the reader a feel for the accuracy of the calculated results.

There are several factors to consider when comparing the results of the two studies. The scheme used in the BRH calculations does not include any collimator scatter. This scattering explains why the two studies do not agree well when the organ of interest is far from the beam. Second, the absorbed dose to an organ was calculated as the absorbed dose averaged over the entire organ. In the experiments, the absorbed dose was measured at the center of an organ. Third, the necessary simplifying assumptions in the BRH calculations were many. Parallel beams replaced a divergent beam, the Monte Carlo radiation 
transport methodology did not include coherent scattering, and entire diagnostic $x$-ray spectra were simulated by five monoenergetic beams. Finally the measured spectra were acquired with configurations (and spectra) which did not always resemble the conditions simulated by the BRH. That the results of two, widely differing, complex studies agreed as well as they do is surprising (and, to this author, pleasing). 


\section{CHAPTER V}

\section{FINAL SUMMARY}

The objectives of this study were the development of a spectrometry system suitable for in-phantom research, demonstration of the feasibility of in-phantom measurements of spectra, and determination of several dosimetric parameters within an anthropomorphic phantom exposed to $x$ rays in a manner simulating medical diagnostic radiological examinations. It is felt that these objectives were met in general. Further, data relevant to the understanding of the transport of radiation within the human body and to the reduction of absorbed dose to patients undergoing $x$-ray examinations were acquired.

In addition to the design and development of the spectrometer, there were a few other technological problems met and overcome in the course of this study. The equipment needed to acquire and process the spectral data was not insignificant. Besides the multichannel analyzer, the use of a large and sophisticated computer system was essential. Second, even though a sodium iodide scintillation spectrometer can be operated at count rates higher than can most other spectrometers, the $x$-ray machine, especially developed for low current operation, was necessary. Without an $x$-ray source having a wide dynamic range $(4.5$ orders of magnitude), this project could not have been performed. These two factors, extensive data handling capability and an $x$-ray machine with extended tube current range, limit the widespread applicability of the spectrometry system developed. 
There were three notable shortcomings of this project. As was discussed previously, the spectral output of the x-ray machine used was not the best simulation of diagnostic $x$-ray spectra. The use of a $x$-ray tube with a low barium content would have helped significantly. Due to budgetary and time constraints, procuring such a tube was not possible for this project. Second,despite the special design and construction, the $\mathrm{NaI}$ detector used in the project did not have a completely uniform angular response. It seems that a better spectrometer could be designed by optimizing the light collection parts of the detector. It is suggested that the $\mathrm{NaI}$ sphere be completely imbedded in a larger polyvinyl toluene light pipe to provide more uniform light collection. Finally, the poor resolution of the detector was a major drawback. Building a small, submersible spectrometer with nearly uniform angular response, good resolution, and the capability to operate at high count rates may not be feasible with today's technology.

The absorbed dose rates, determined from the measured fluence distributions, compared reasonably well with the few ionization chamber measurements and with the results of Monte Carlo calculations. The $\pm 15 \%$ agreement with ionization chamber measurements is close to the accuracy that is expected of many dosimetric techniques excepting those conducted under ideal conditions. The agreement with the results of the BRH Monte Carlo calculations does not appear good prima facie. However, there are several factors that make this comparison seem much better. The beam configuration, spectrum, and location of measurements did not exactly correspond to the respective quantities in the BRH calculations. Also the BRH estimations of absorbed dose were based on Monte Carlo 
calculations, some of which had large statistical uncertainty. Further the measurements conducted in this study have a nontrivial, estimated, experimental error.

The data acquired in this study may be of use to a variety of researchers. However, potential users are cautioned against indiscriminately applying the results of this study before considering the suitability of the MR. ADAM phantom and the degree to which the spectra used in this study simulate the spectra in the situation being studied. The MR. ADAM phantom has several regions of poor simulation including the head, the upper chest area, and the arm regions. Anyone specifically interested in these regions may find the MR. ADAM phantom and the results of this study unsuitable. Also, the results of this study may not be suitable to the study of a particular patient. Obviously, there is no patient quite like MR. ADAM, and the dissimilarity between this phantom and a particular patient will have to be evaluated in each case. The spectra of the $x$-ray machine used in this study only approximate spectra typical of diagnostic radiology. Further, depending on type of rectification, actual inherent filtration, and other factors, any particular $x$-ray machine may produce $x$ rays with a spectral distribution and other characteristics that differ from "typical". It is suggested that at least the half value layer of the actual x-ray machine being studied be compared with that of the $x$-ray machine used in this study. Of course, direct determination of the output spectra of an $x$-ray machine would be the test indication of the similarity and differences between its spectra and the ones used in this study. However, since direct determination of the spectra of an x-ray machine by means of a high 
resolution spectrometer is often difficult and expensive, determination of half value layer may be the only practical means of comparison. If the half value layers of the $x$-ray beams being considered are similar to the ones in this study, correction for exposure rate differences between the $x$-ray machines should lead to valid applications of the data presented in this text.

Within the limits imposed by the accuracy of the simulations, the rcsolution of the detector, and the other experimental error's, the data acquired in this study revealed some interesting information concerning the transport of diagnostic $x$-rays in the human body. By far, the most important factor affecting spectral shape within the phantom was the generating potential. The second most important factor was the medium in which the spectrum was measured. The spectra measured in the spine had a higher average energy than those measured in soft-tissue. Depth of penetration into the phantom affected spectral shape. Over the range of depths for which spectra were acquired in the direct beam, the average energy of the measured spectra increased. However, the increase was not dramatic. At $100 \mathrm{kVcp}$ generating potential with a $10 \times 10 \mathrm{~cm}$ beam centered on the detector, the average energy of the measured spectrum increased frnm $54 \mathrm{keV}$ to $56 \mathrm{keV}$ as the detector was moved from $\mathrm{y}=1$ to $y=0$ (a change of $4 \mathrm{~cm}$ ). However, the shape of the spectrum scattered from a $10 \times 10 \mathrm{~cm}$ beam as a function of distance between the beam center and center of the detector did not change significantly over a distance of $31 \mathrm{~cm}$.

Data from the exposure configurations in which shielding was used demonstrated two effects relevant to the reduction of absorbed dose to 
radiosensitive regions in patients undergoing $x$-ray examinations. The first effect is that of collimator scatter. Comparing the data acquired under configurations that were identical in all manners except shielding showed that in many cases, the effect of the shielding was significant. For regions of interest outside the useful beam, the radiation scattered from the collimator was of comparable size to the radiation scattered from the useful beam within the phantom. It seems that the amount of radiation scattered would depend on the design of the collimator. Since a therapy-type collimator was used in this study, it is not recommended that the data acquired be applied directly to consideration of this effect. However, it is hoped that the trends in the data concerning collimator scatter might create some interest in this area. Additional experimentation would better define the nature of the collimator scatter, and subsequent calculational investigations might produce a design for collimators optimized to reduce scatter. In addition to reduction of absorbed dose to patients, reduction of collimator scatter would improve the quality of radiographs.

The second effect, relating to the reduction of absorbed dose, is merely a different view of the collimator scattering. Since collimator scattering was demonstrated to be significant, shielding body areas outside the useful beam would reduce the absorbed dose to radiosensitive areas such as blood-forming organs, thyroid, lens of the eyes, and reproductive organs. Data acquired in this study indicated that al though the amount of absorbed dose reduction caused by shielding was often small, the fractional dose reduction was always significant. 
Annually, millions of people in this country alone undergo $x$-ray examinations. When one considers that all absorbed dose to regions outside the useful beam is "wasted" and has the potential for doing only harm, using all reasonable methods to minimize absorbed dose to such regions in the millions of patients seems only reasonable. The results of this study indicate that shielding all body areas outside the area to be examined would be an easy way to help keep the risk from medical diagnostic $x$-rays "as low as practicable." 
APPENDIX A

\section{MEASURED SPECTRA}

Presented in this Appendix are the unfolded spectra for 147 combinations of detector location, generating potential, and beam configuration. A summary of beam configurations and detector locations is given in Figures 22-28. In addition, the spectra acquired with the testes region shielded with $0.16 \mathrm{~cm}$ of lead are presented immediately following the spectra for the corresponding unshielded case. 


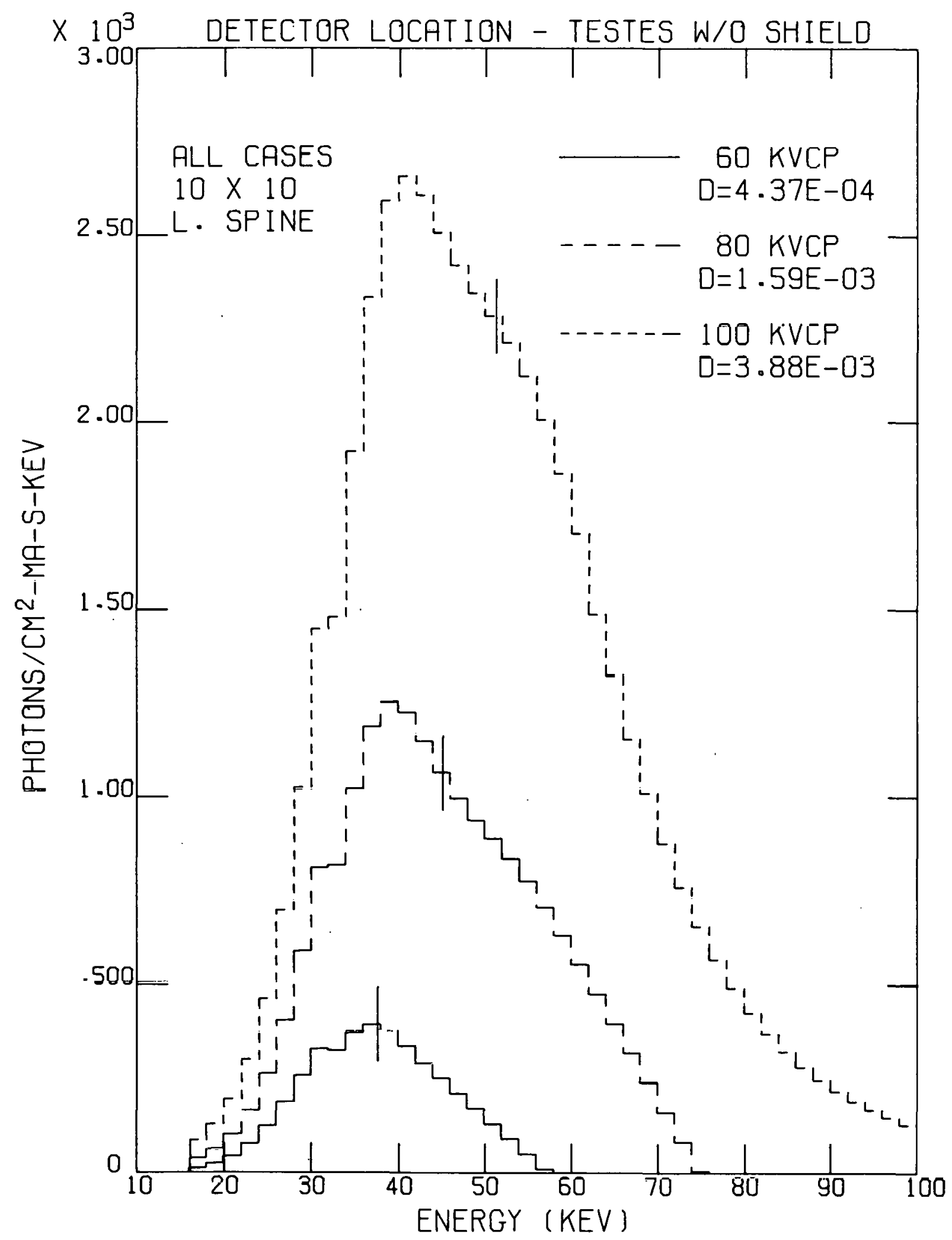

Figure 48. Spectrum at the Testes without Shield for Lower Spine. Vertical ticks indicate mean energy. Field size is in $\mathrm{cm}$. D is the absorbed dose per unit current per unit time (mrad/mA-s). 


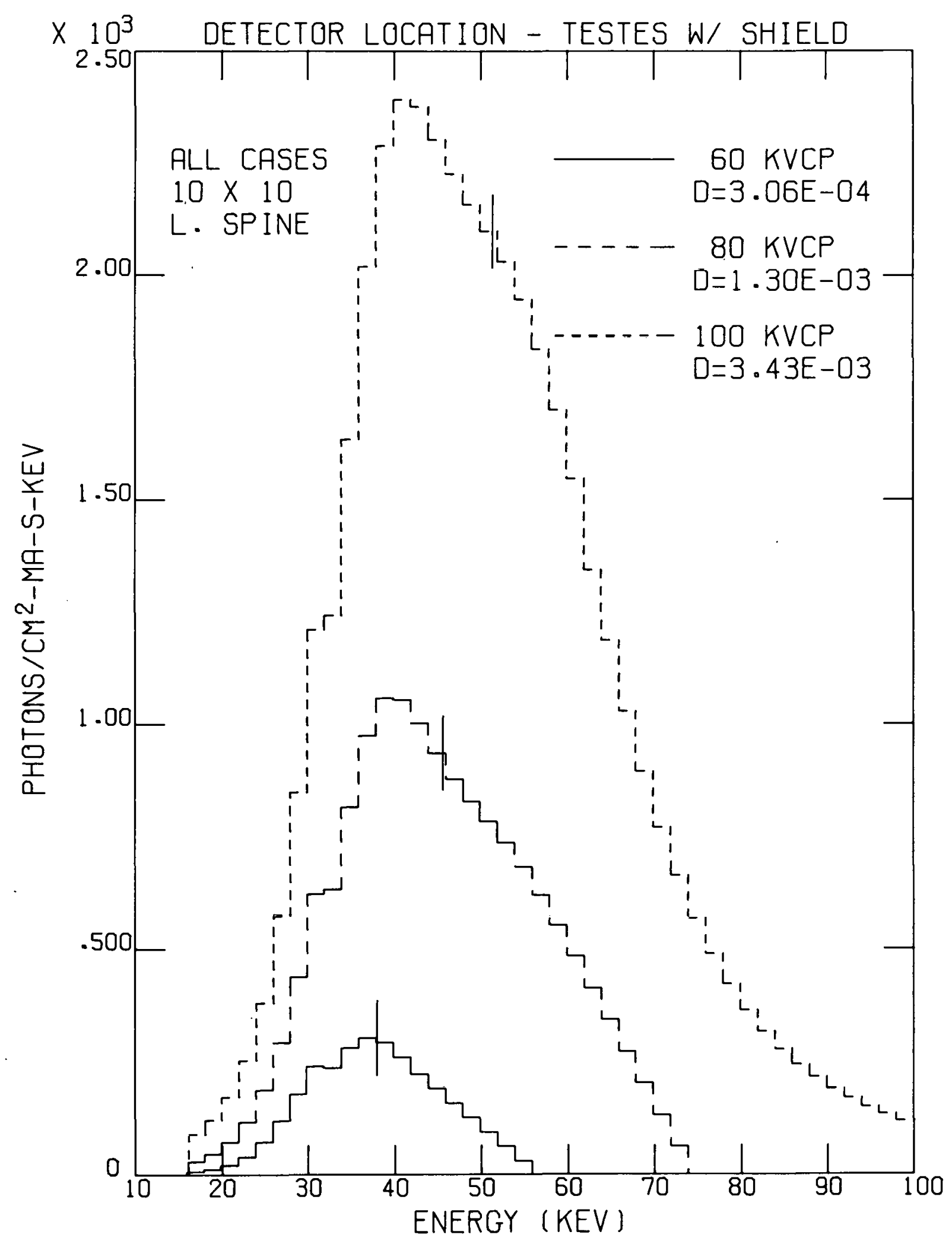

Figure 49. Spectrum at the Testes with Shield for Lower Spine Exam. Vertical ticks indicate mean encrgy. Field size is in $\mathrm{cm}$. $D$ is the absorbed dose per unit current per unit time ( $\mathrm{mrad} / \mathrm{mA}-\mathrm{s})$. 


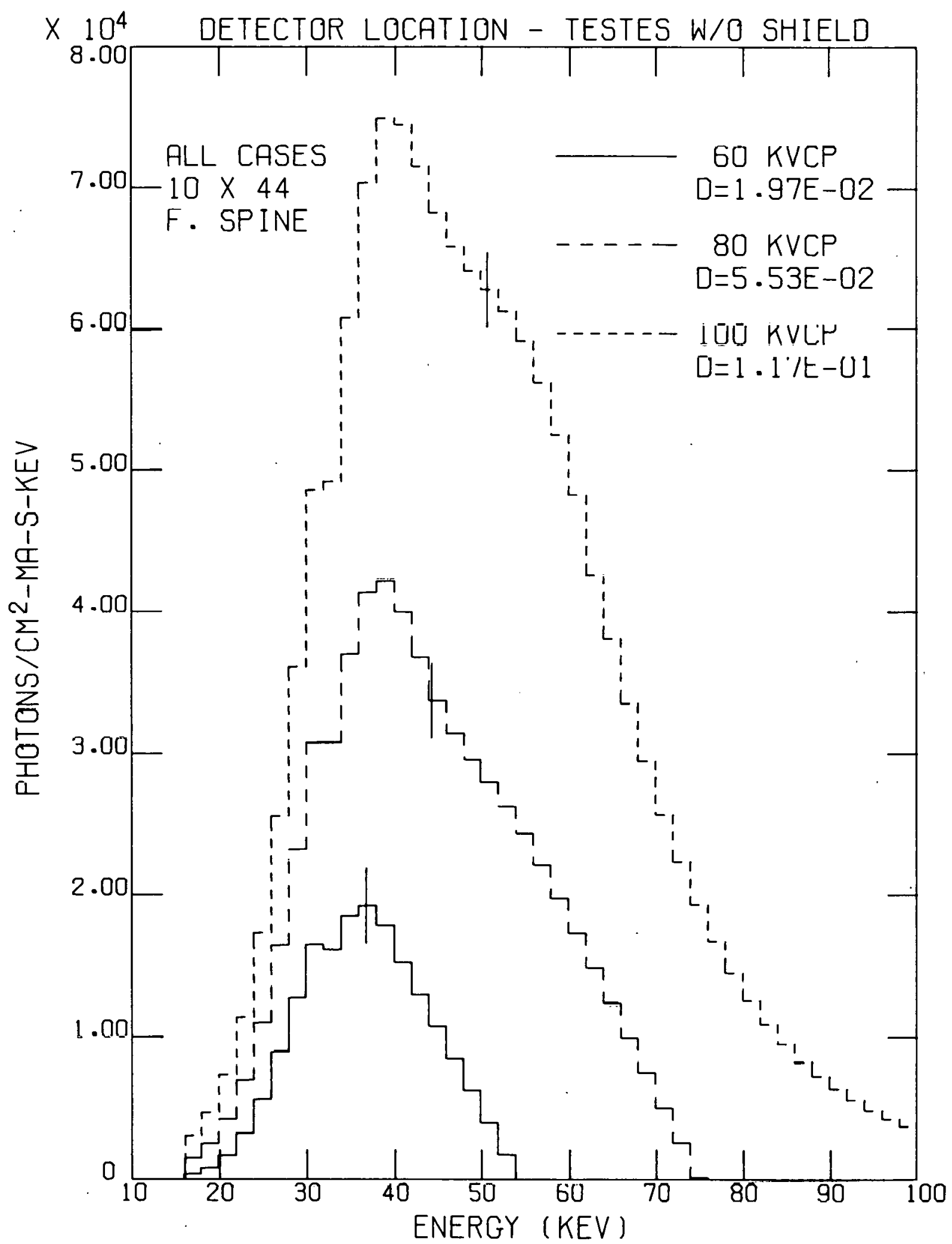

Figure 50. Spectrum at the Testes without Shield for Full Spine Exam. Vertical ticks indicate mean energy. Field size is in $\mathrm{cm}$. $D$ is the absorbed dose per unit current per unit time $(\mathrm{mrad} / \mathrm{mA}-\mathrm{s})$. 


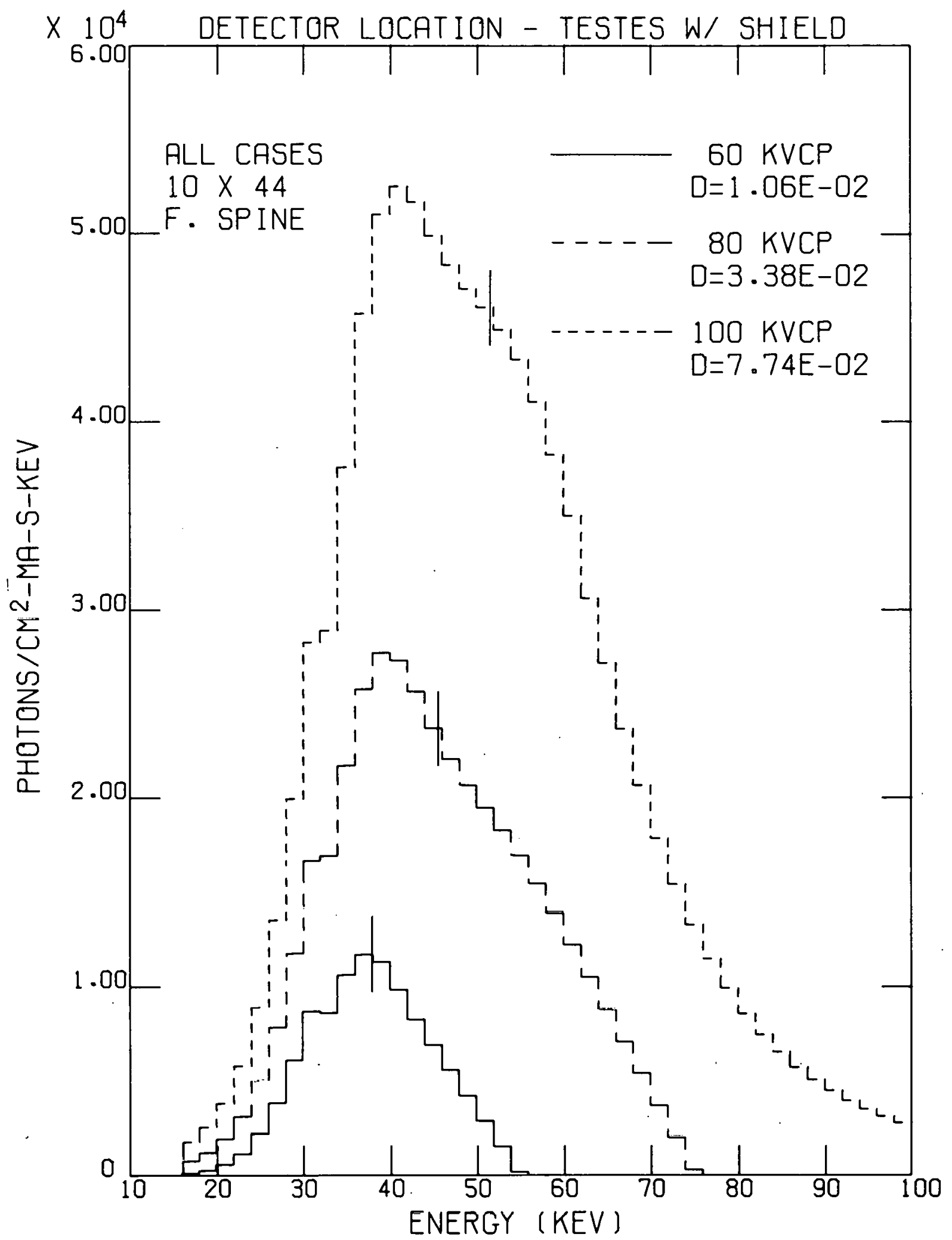

Figure 51. Spectrum at the Testes with Shield for Full Spine Exam. Vertical ticks indicate mean energy. Field size is in $\mathrm{cm}$. $D$ is the absorbed dose per unit current per unit time (mrad/mA-s). 


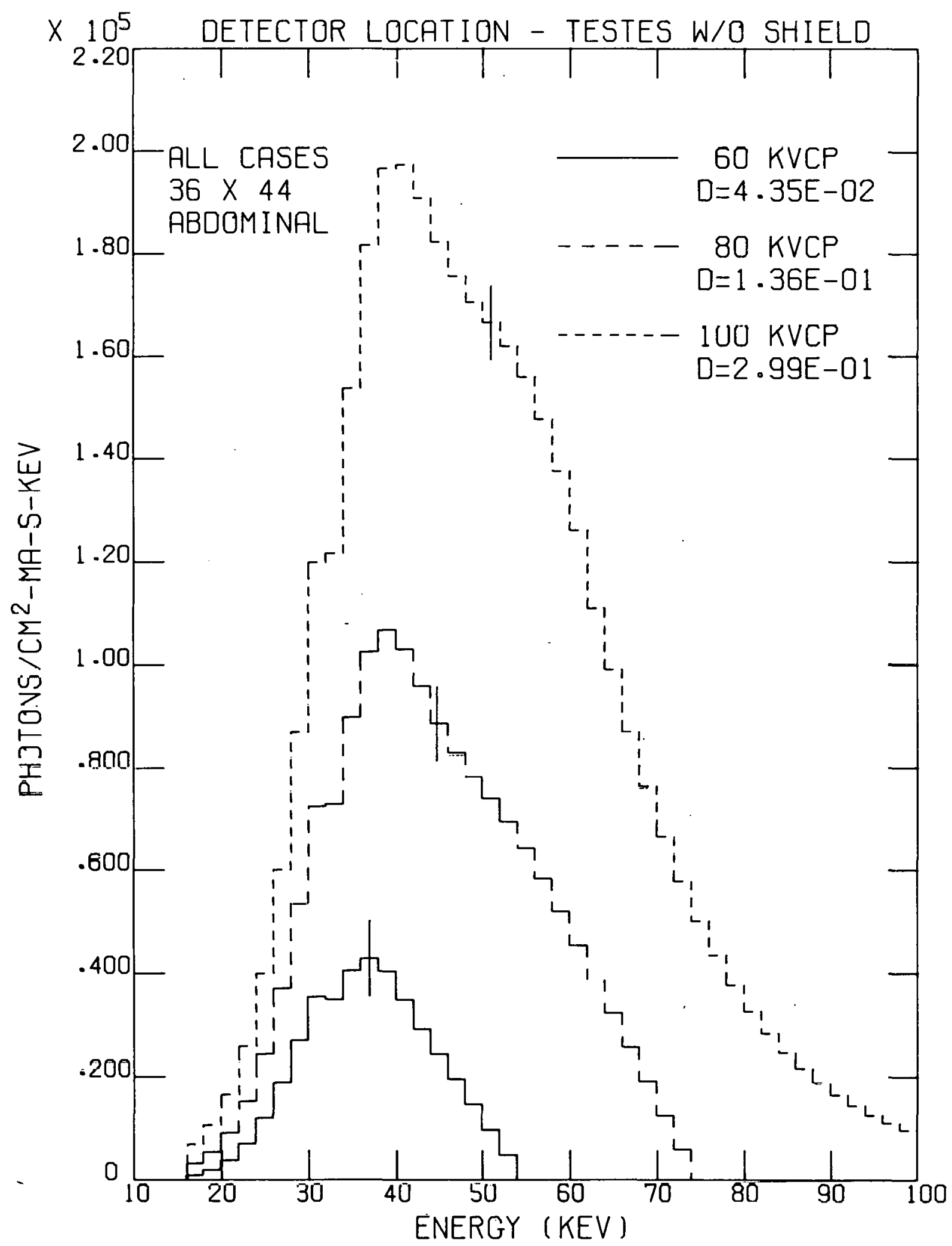

Figure 52. Spectrum at the Testes without Shield for Abdominal Exam. Vertical ticks indicate mean energy. Field size is in $\mathrm{cm}$. $D$ is the absorbed dose per unit current per unit time $(\mathrm{mrad} / \mathrm{mA}-\mathrm{s})$. 


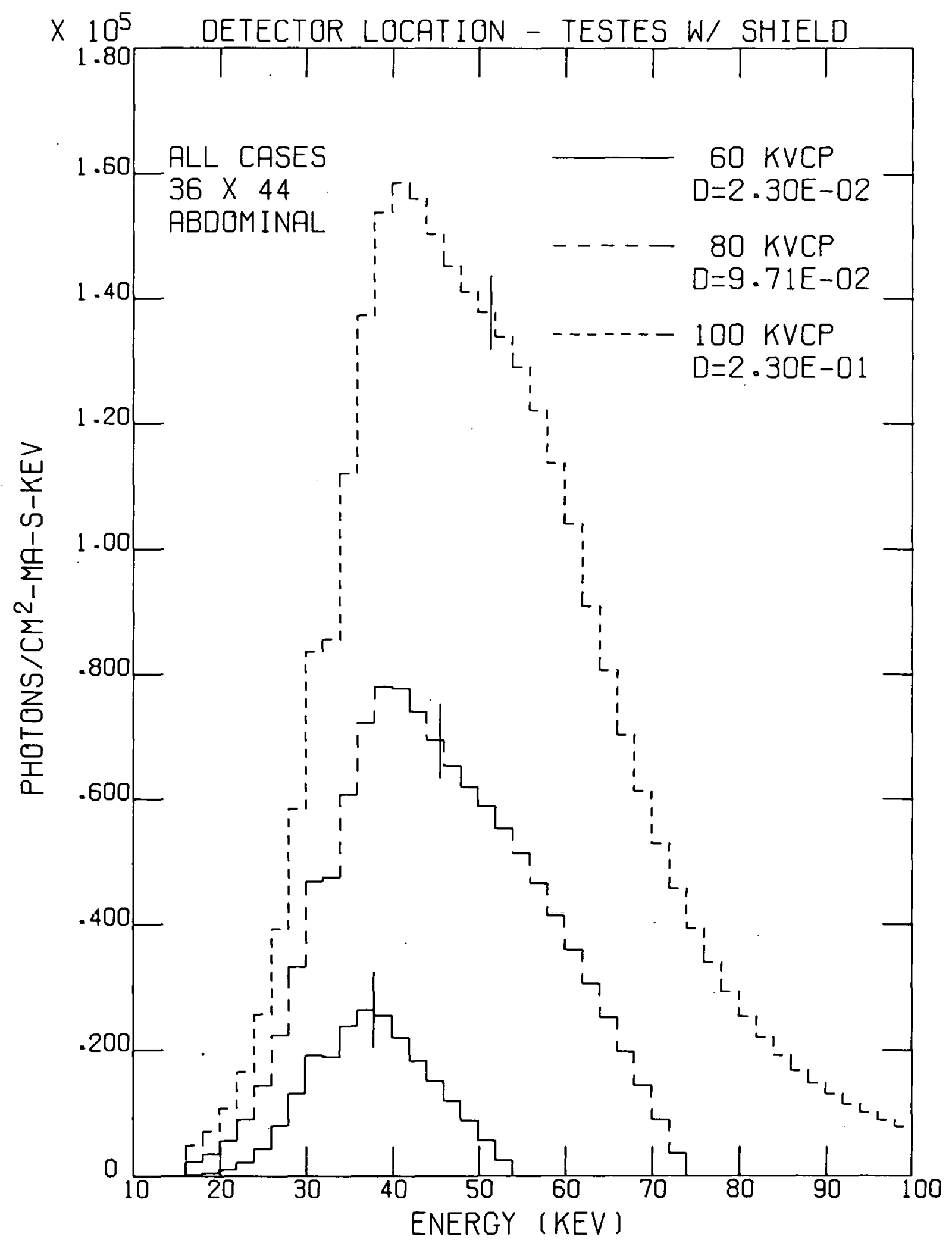

Figure 53. Spectrum at the Testes with Shield for Abdominal Exam. Vertical ticks indicate mean energy. Field size is in $\mathrm{cm}$. $D$ is the absorbed dose per unit current per unit time (mrad/mA-s). 


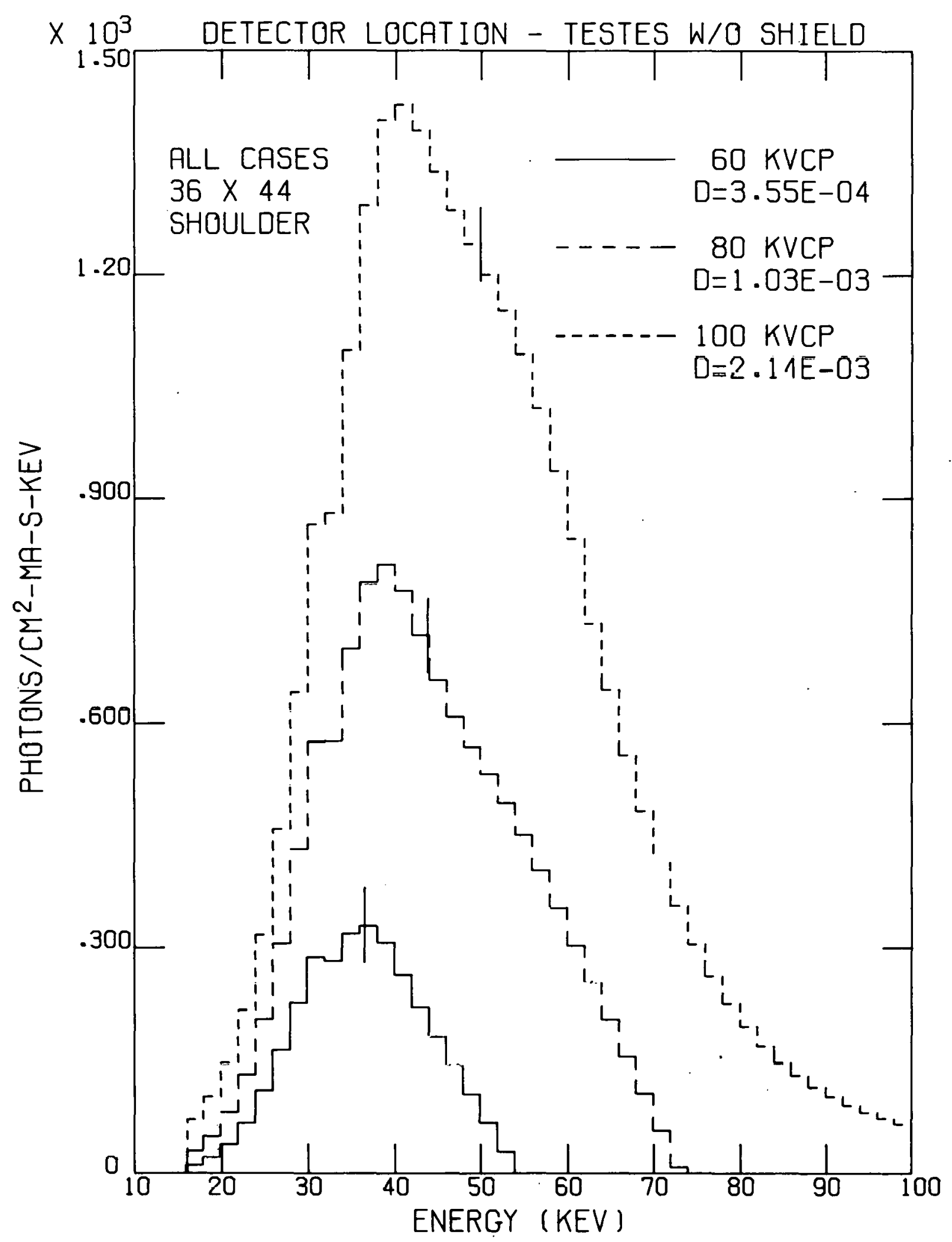

Figure 54. Spectrum at the Testes without Shield for Shoulder Exam. Vertical ticks indicate mean energy. Field size is in $\mathrm{cm}$. $D$ is the absorbed dose per unit current per unit time $(\mathrm{mrad} / \mathrm{mA}-\mathrm{s})$. 


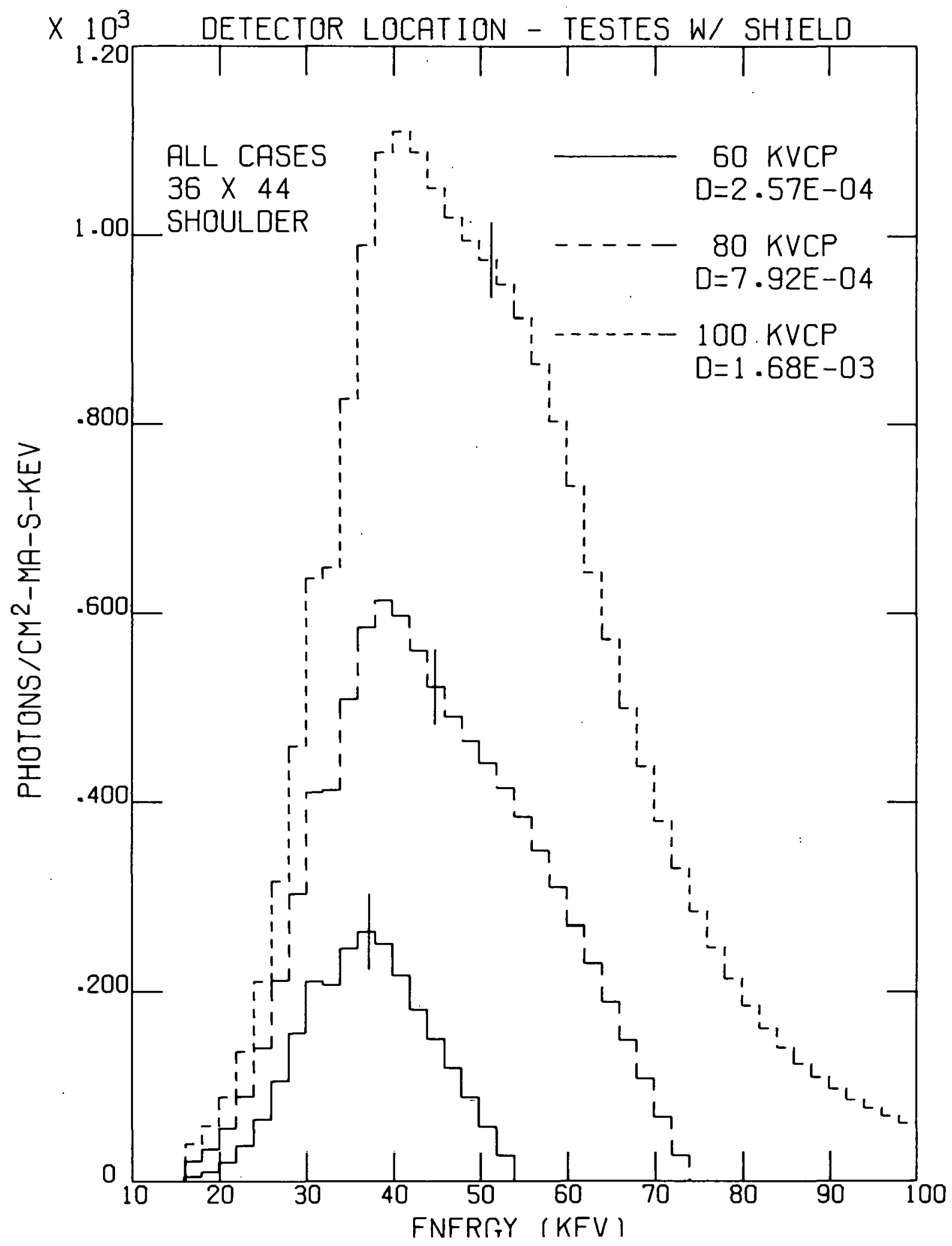

Figure 55. Spectrum at the Testes with Shield for Shoulder Exam. Vertical ticks indicate mean energy. Field size is in $\mathrm{cm}$. $D$ is the absorbed dose per unit current per unit time (mrad/mA-s). 


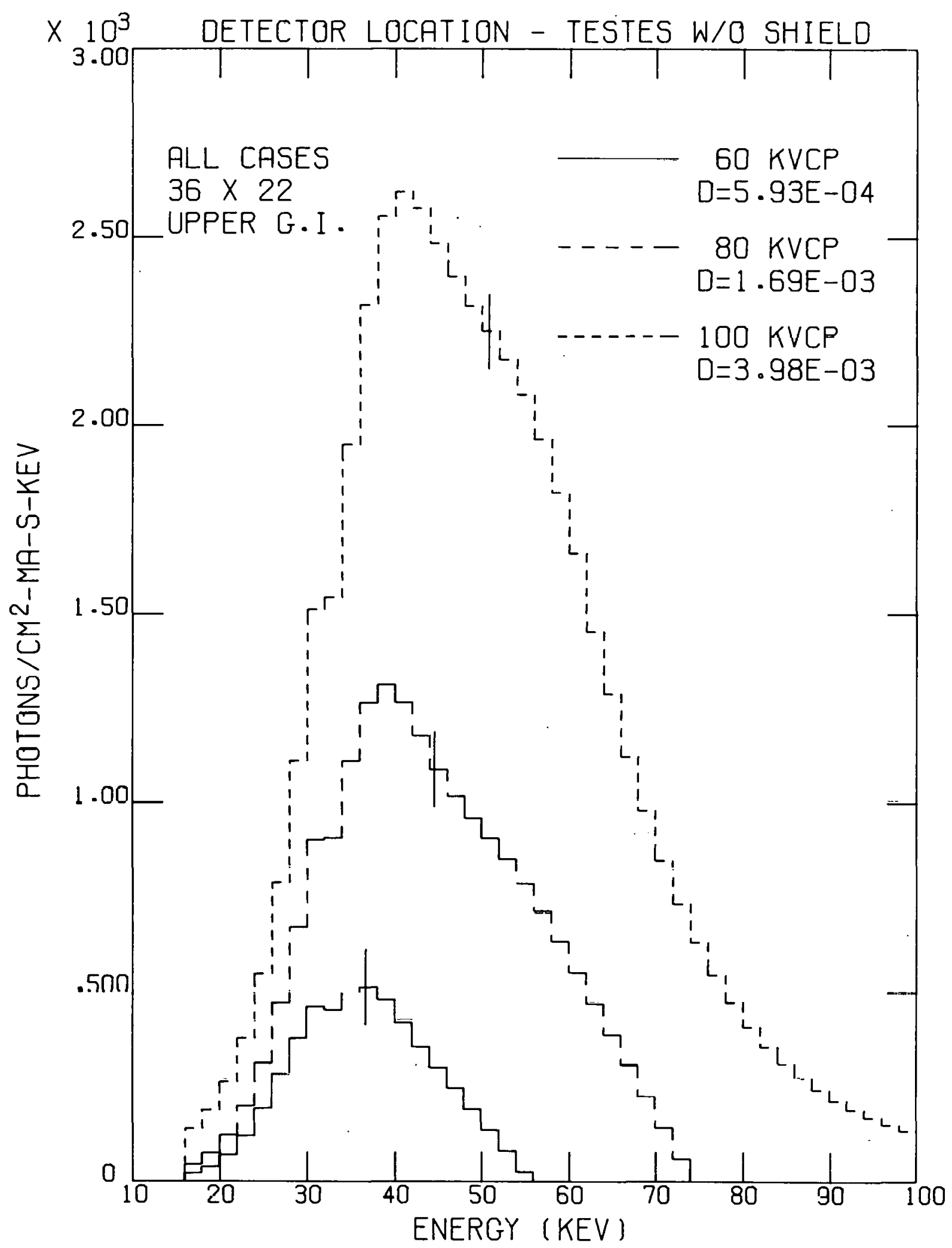

Figure 56. Spectrum at the Testes without Shield for Upper G.I. Exam. Vertical ticks indicate mean energy. Field size is in $\mathrm{cm}$. $D$ is the absorbed dose per unit current per unit time (mrad/mA-s). 


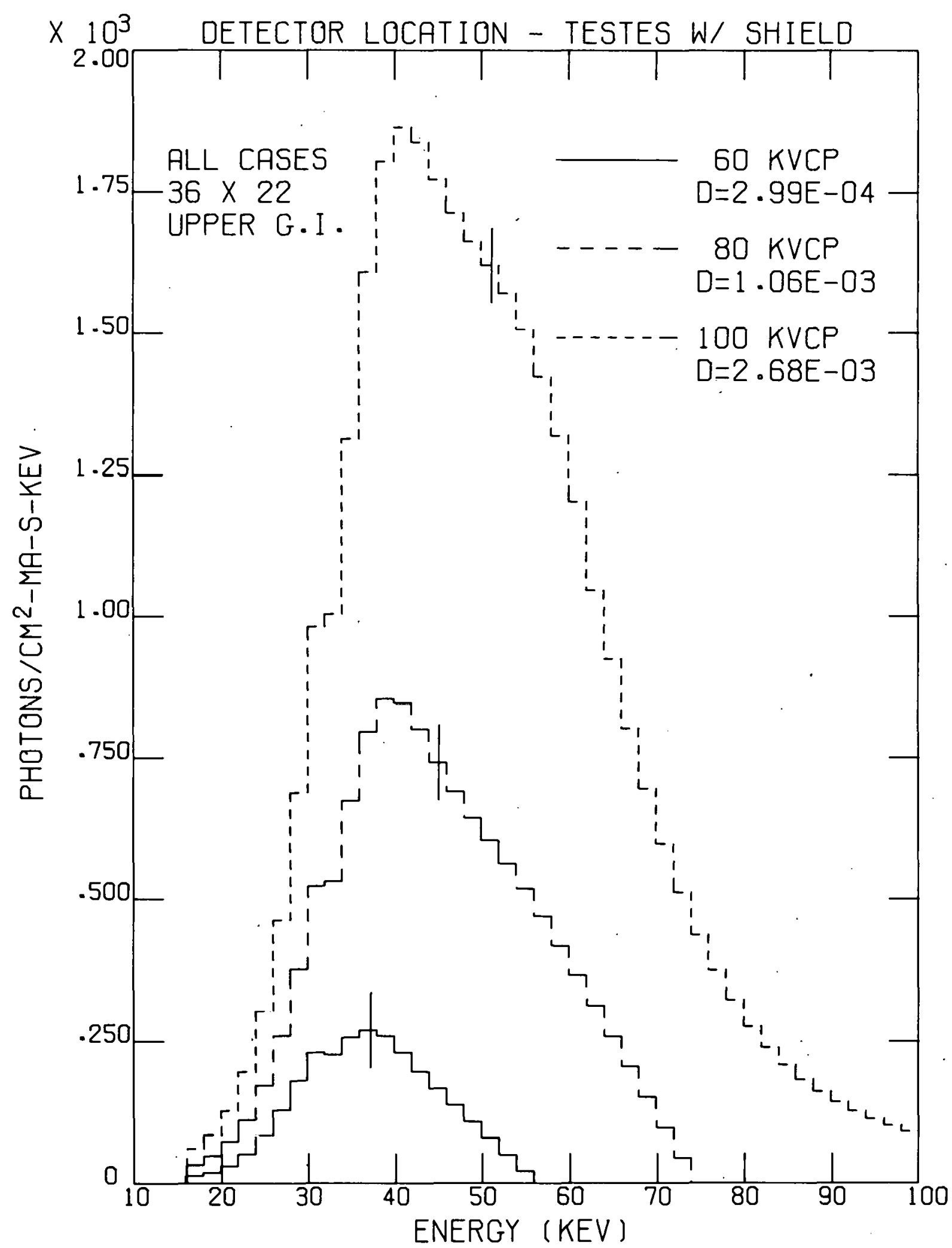

Figure 57. Spectrum at the Testes with Shield for Upper G.I. Exam. Vertical ticks indicate mean energy. Field size is in $\mathrm{cm}$. $D$ is the absorbed dose per unit current per unit time (mrad/mA-s). 


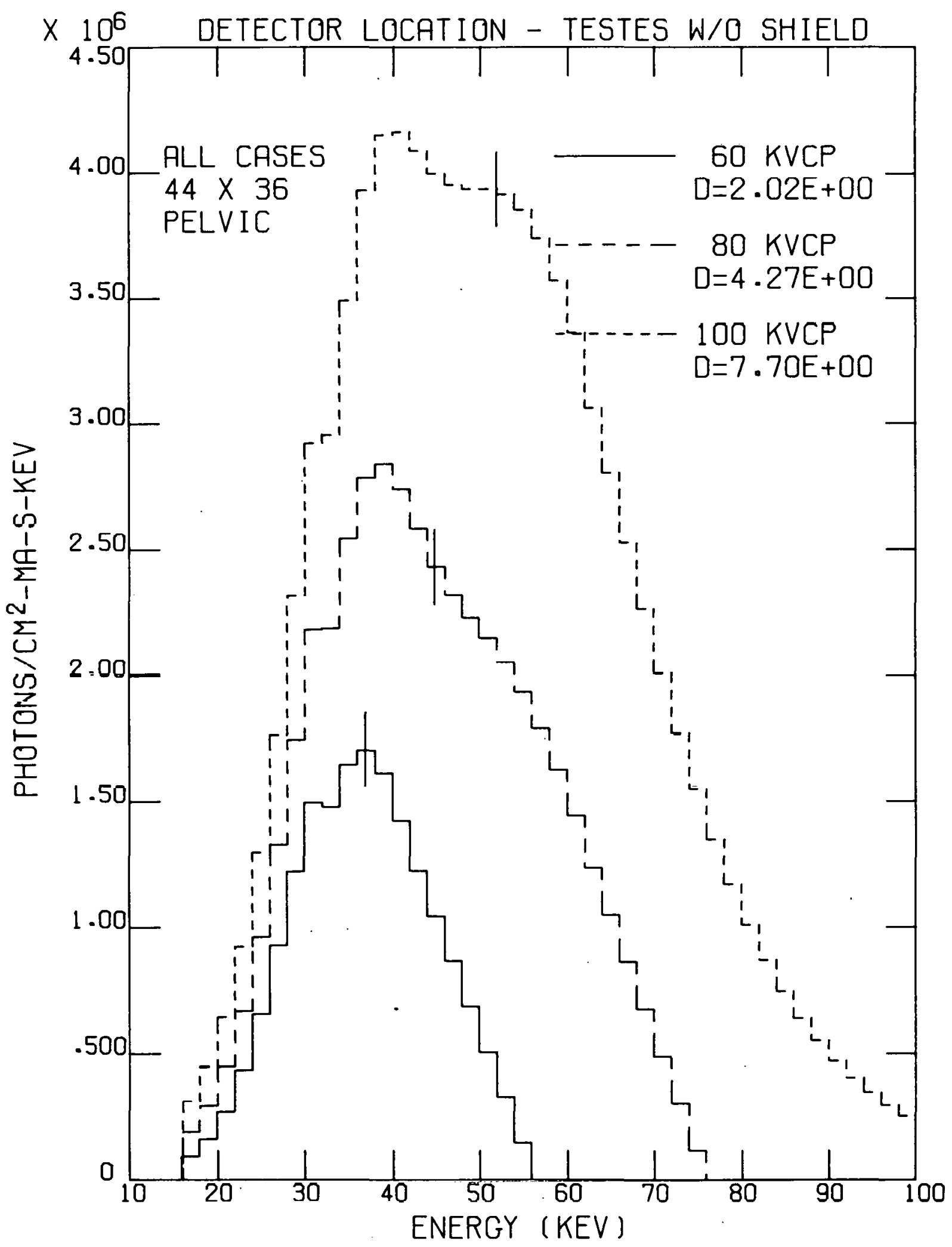

Figure 58. Spectrum at the Testes without Shield for Pelvic Exam. Vertical ticks indicate mean energy. Field size is in $\mathrm{cm}$. $D$ is the absorbed dose per unit current per unit time (mrad/mA-s). 


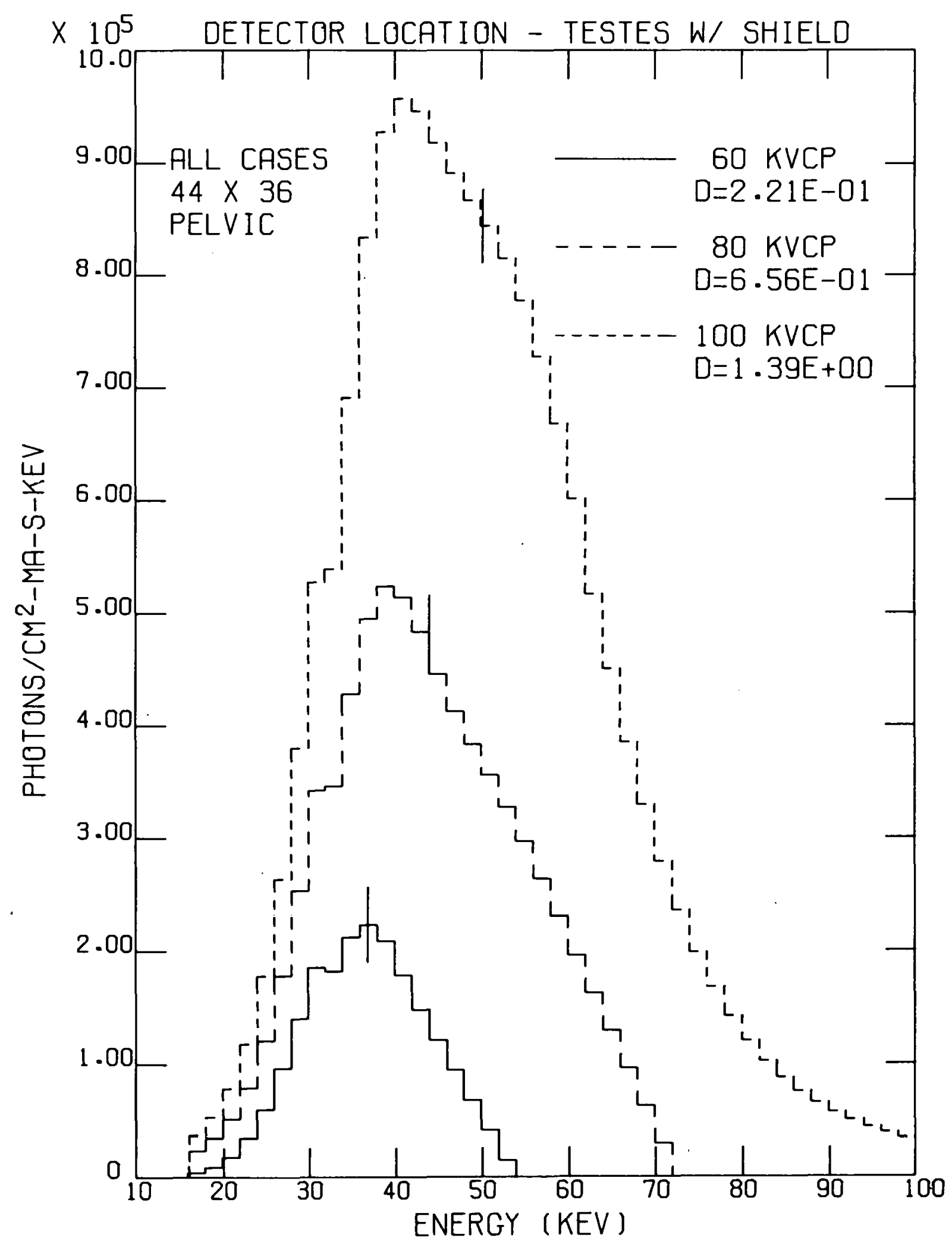

Figure 59. Spectrum at the Testes with Shield for Pelvic Exam. Vertical ticks indicate mean energy. Field size is $\mathrm{in} \mathrm{cm}$. $D$ is the absorbed dose per unit current per unit time ( $\mathrm{mrad} / \mathrm{mA}-\mathrm{s})$. 


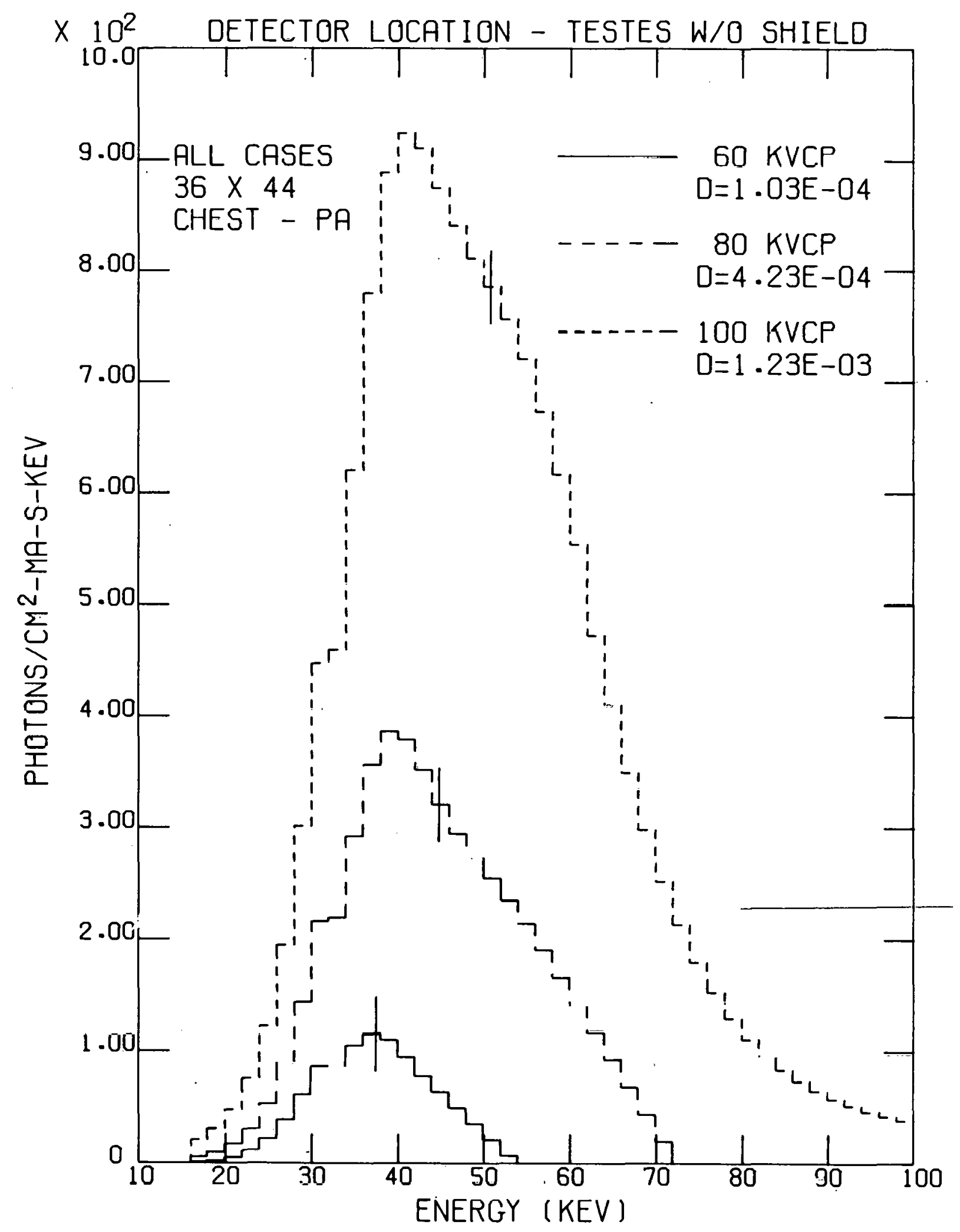

Figure 60. Spectrum at the Testes without Shield for Chest Exam. Vertical ticks indicate mean energy. Field size is in $\mathrm{cm}$. $D$ is the absorbed dose per unit current per unit time $(\mathrm{mrad} / \mathrm{mA}-\mathrm{s})$. 


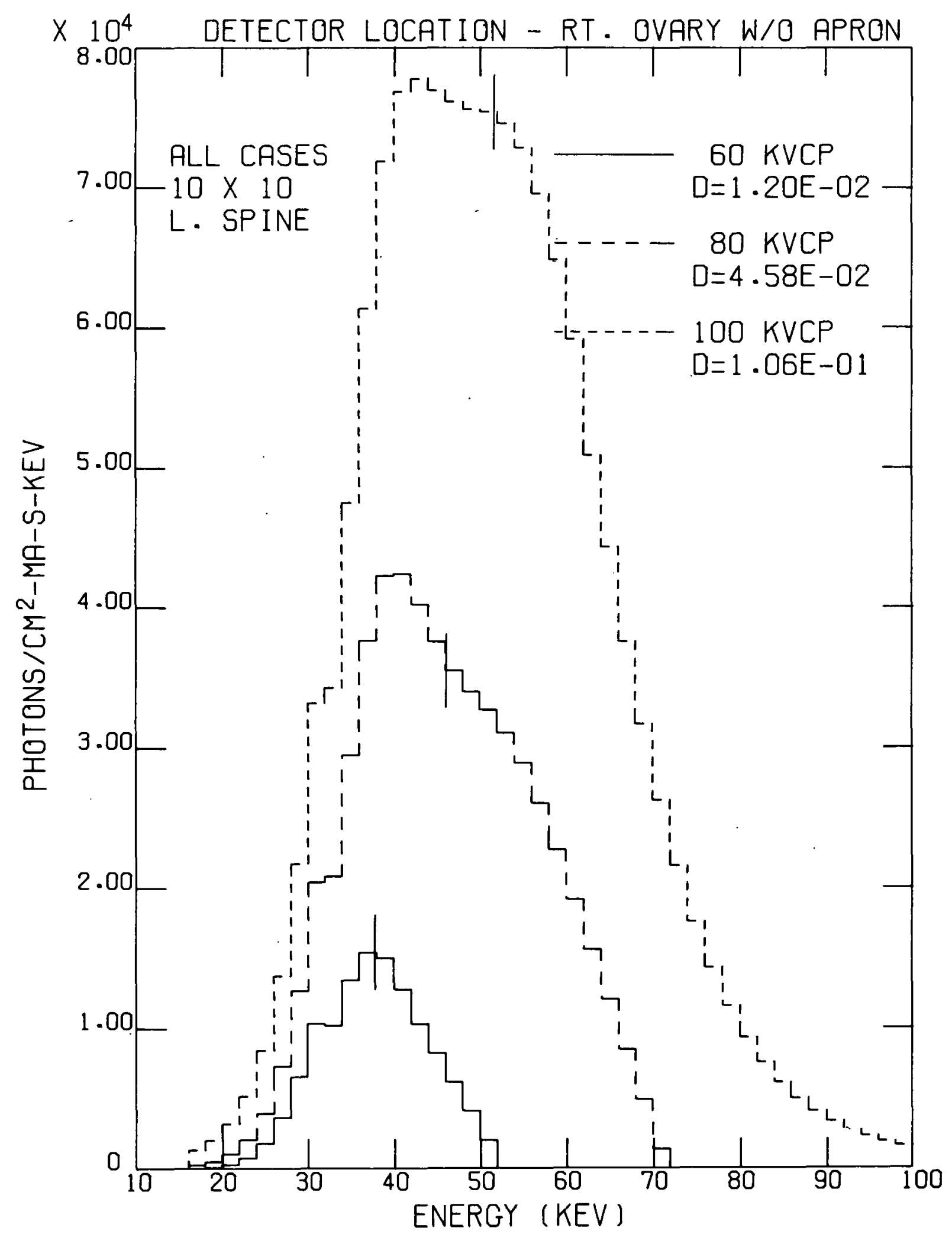

Figure 61. Spectrum at the Right Ovary for Lower Spine Exam. Vertical ticks indicate mean energy. Field size is in $\mathrm{cm}$. D is the absorbed dose per unit current per unit time (mrad/mA-s). 


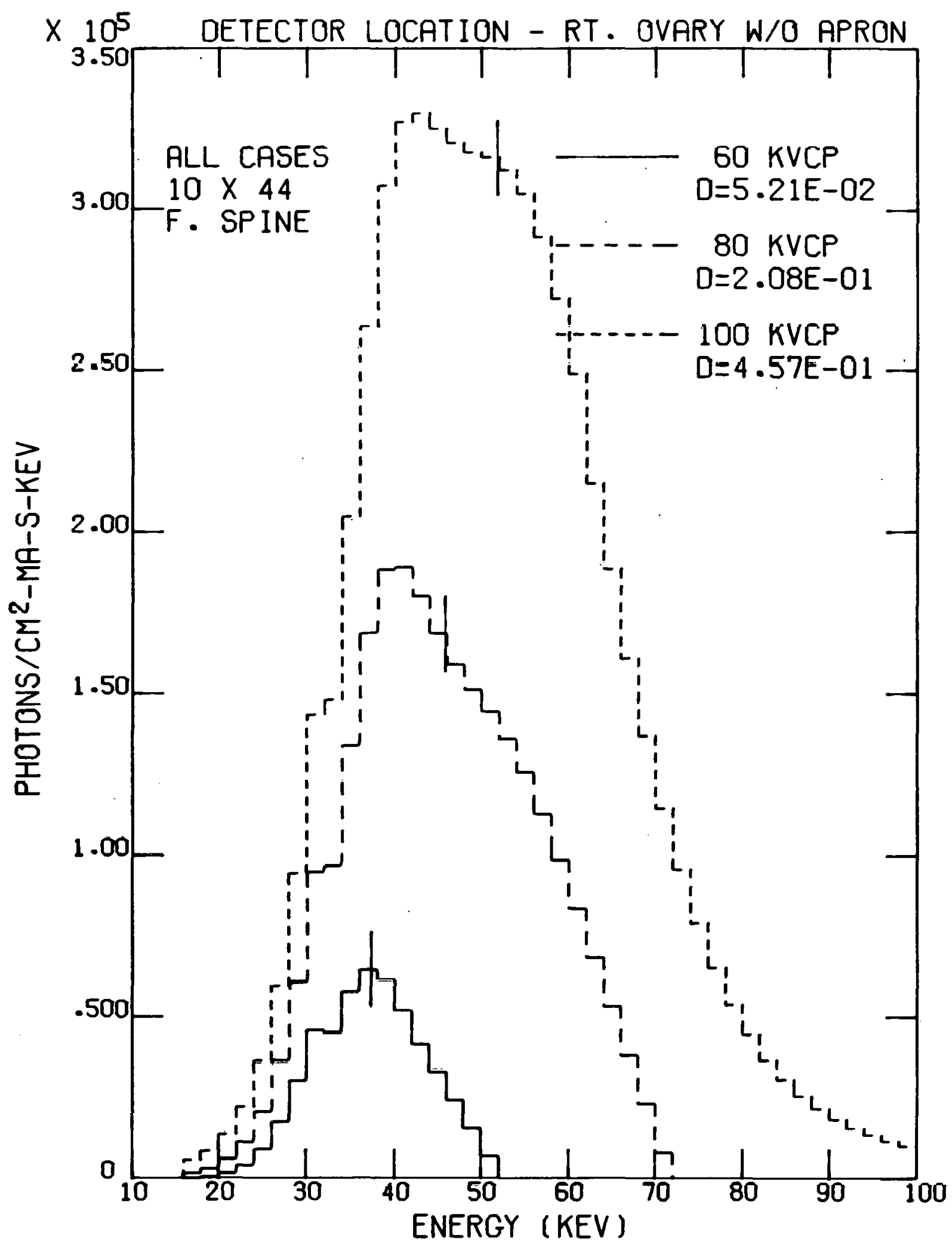

Figure 62. Spectrum at the Right Ovary for Full Spine Exam. Vertical ticks indicate mean energy. Field size is in $\mathrm{cm}$. D is the absorbed dose per unit current per unit time (mrad/mA-s). 


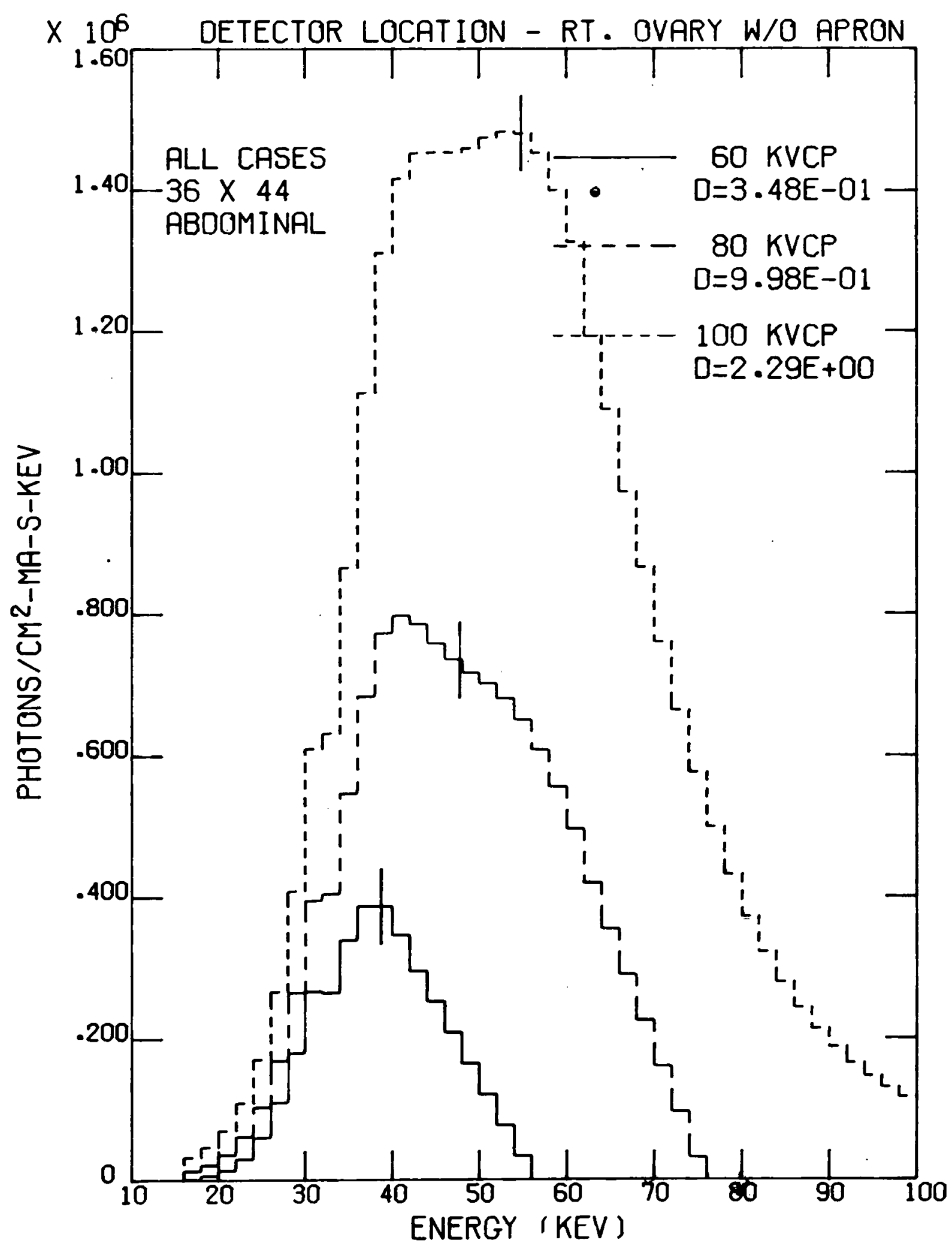

Figure 63. Spectrum at the Right Ovary for Abdominal Exam. Vertical ticks indicate mean energy. Field size is $\mathrm{fn} \mathrm{cm}$. D is the absorbed dose per unit current per unit time (mrad/mA-s). 


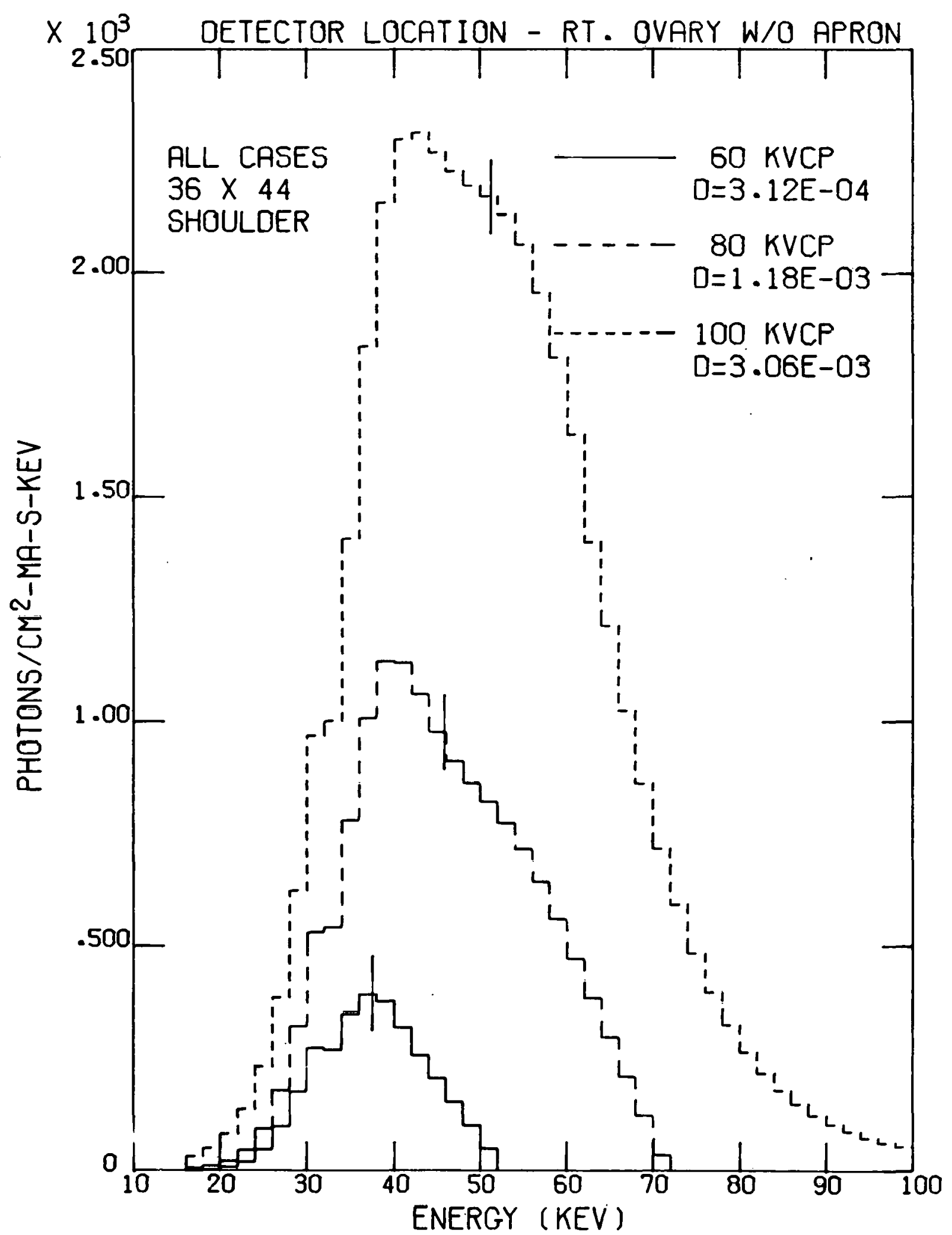

Figure 64. Spectrum at the Right Ovary for Shoulder Exam. Vertical ticks indicate mean energy. Field size is in $\mathrm{cm}$. D is the absorbed dose per unit current per unit time (mrad/mA-s). 


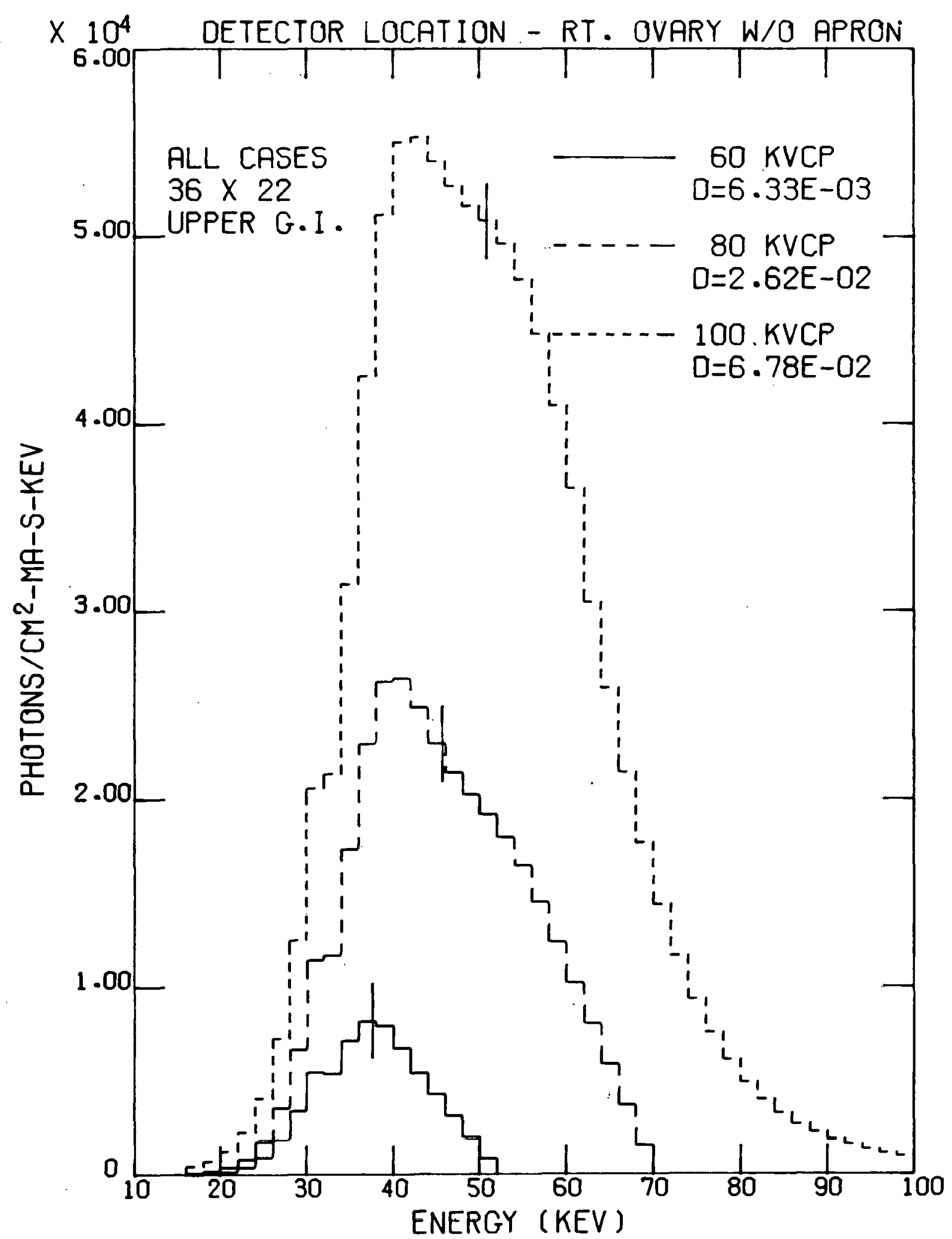

Figure 65. Spectrum at the Right Ovary for Upper G.I. Exam. Vertical ticks indicate mcan energy. Field size is in $\mathrm{cm}$. D is the absorbed dose per unit current per unit time (mrad/mA-s). 


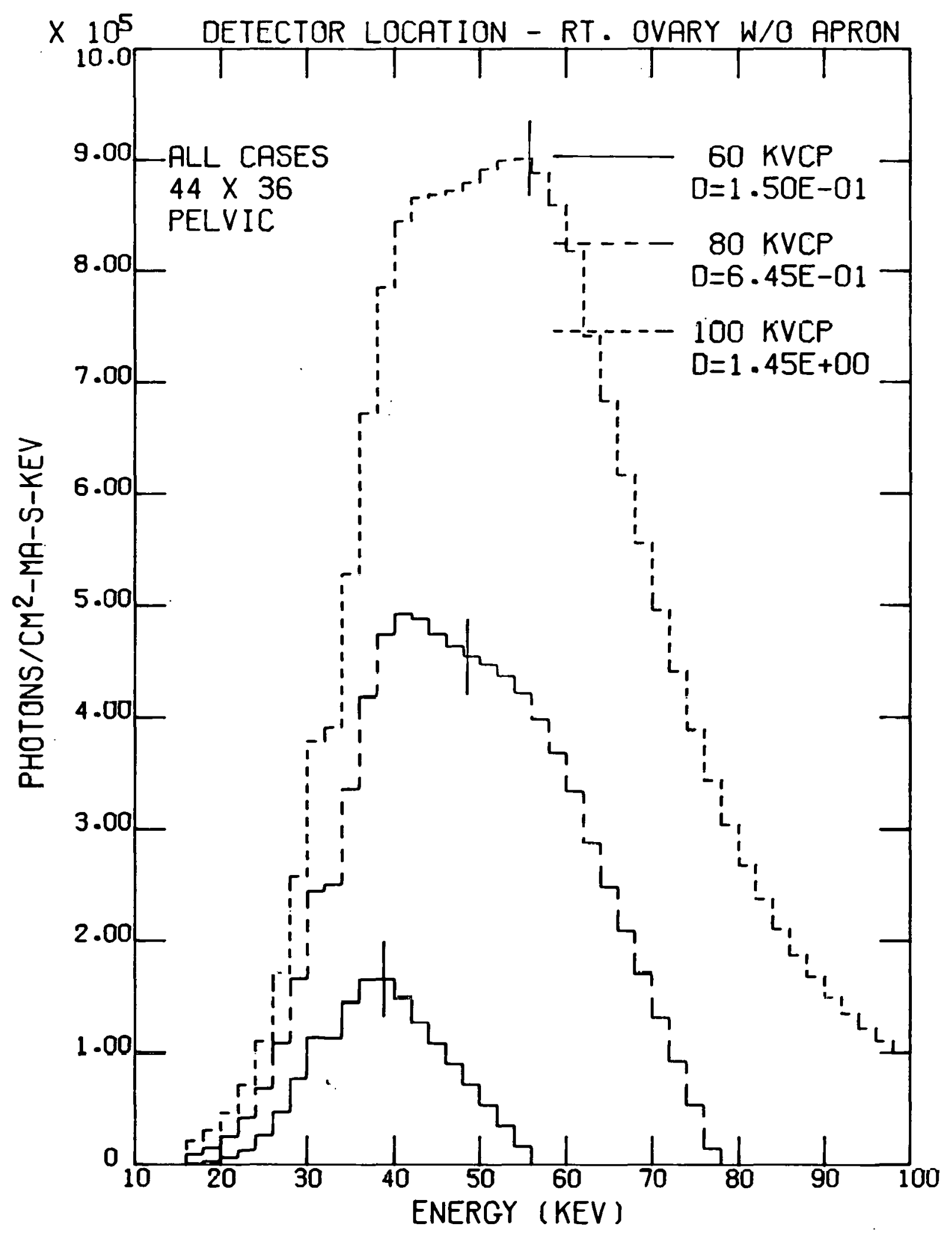

Figure 66. Spectrum at the Right Ovary for Pelvic Exam. Vertical ticks indicate mean energy. Field size is in $\mathrm{cm}$. $D$ is the absorbed dose per unit current per unit time (mrad/mA-s). 


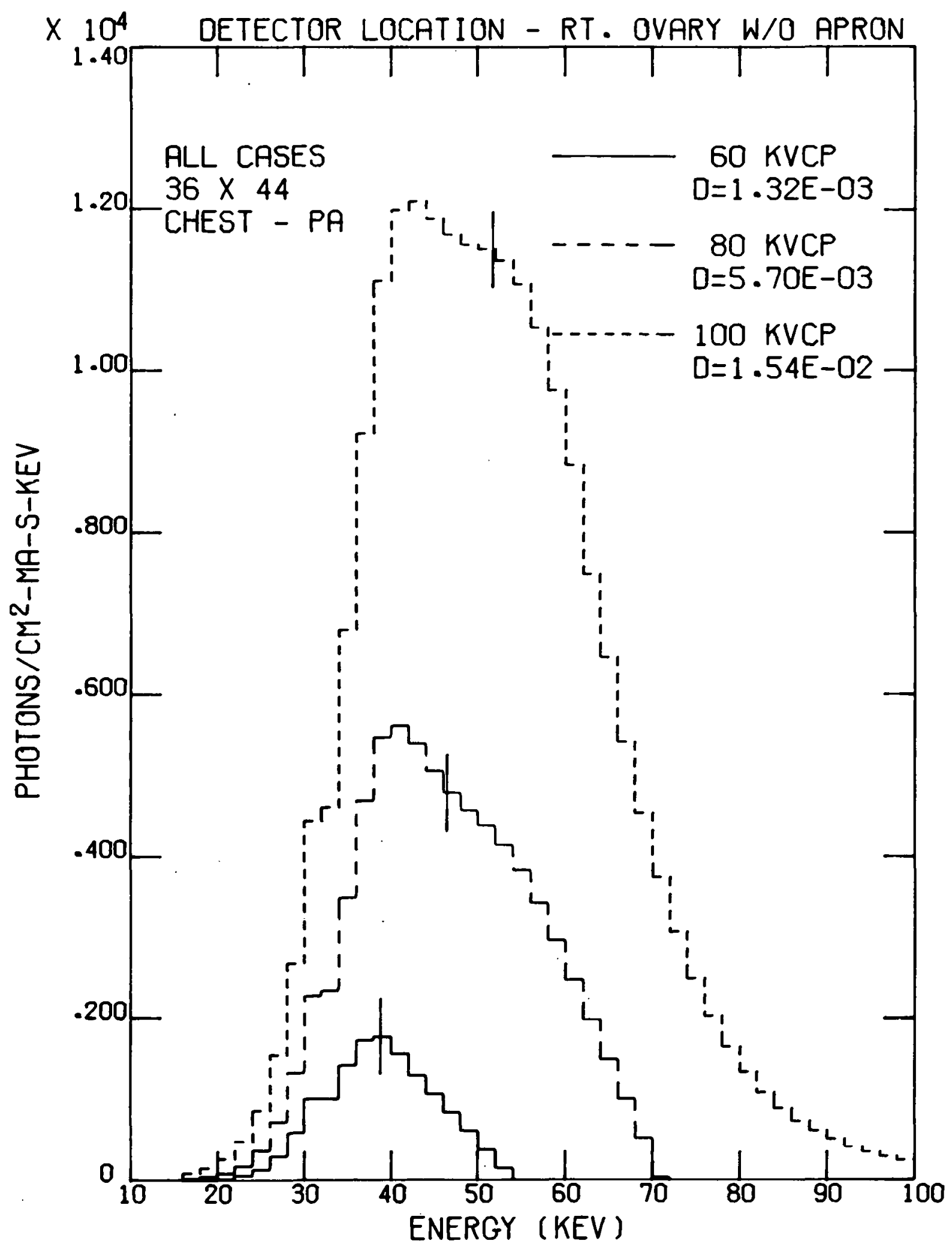

Figure 67. Spectrum at the Right Ovary for Chest Exam. Vertical ticks indicate mean energy. Field size is in $\mathrm{cm}$. $D$ is the absorbed dose per unit current per unit time (mrad/mA-s). 


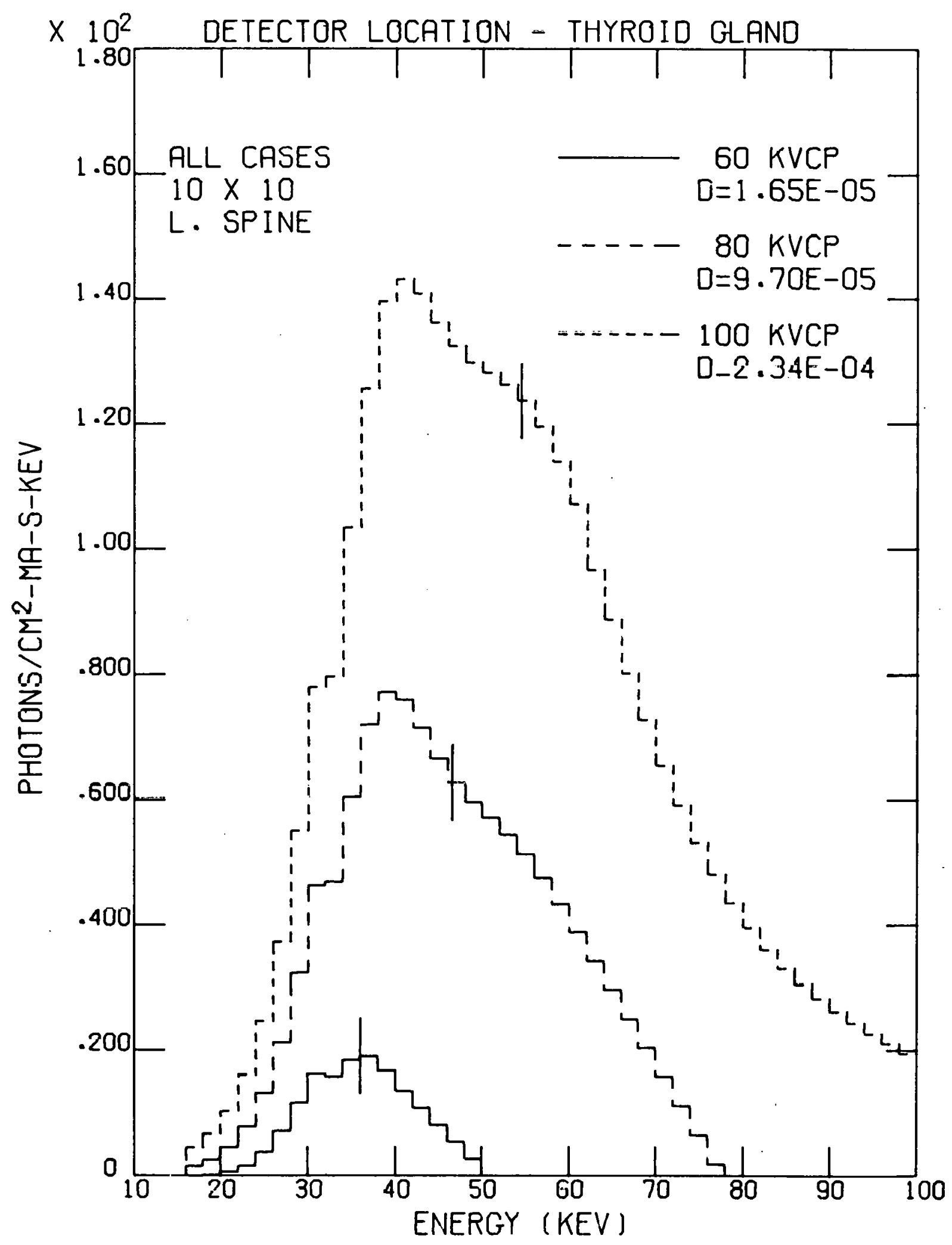

Figure 68. Spectrum at the Thyroid Gland for Lower Spine Exam. Vertical ticks indicate mean energy. Field size is in $\mathrm{cm}$. D is the absorbed dose per unit current per unit time (mrad/mA-s). 


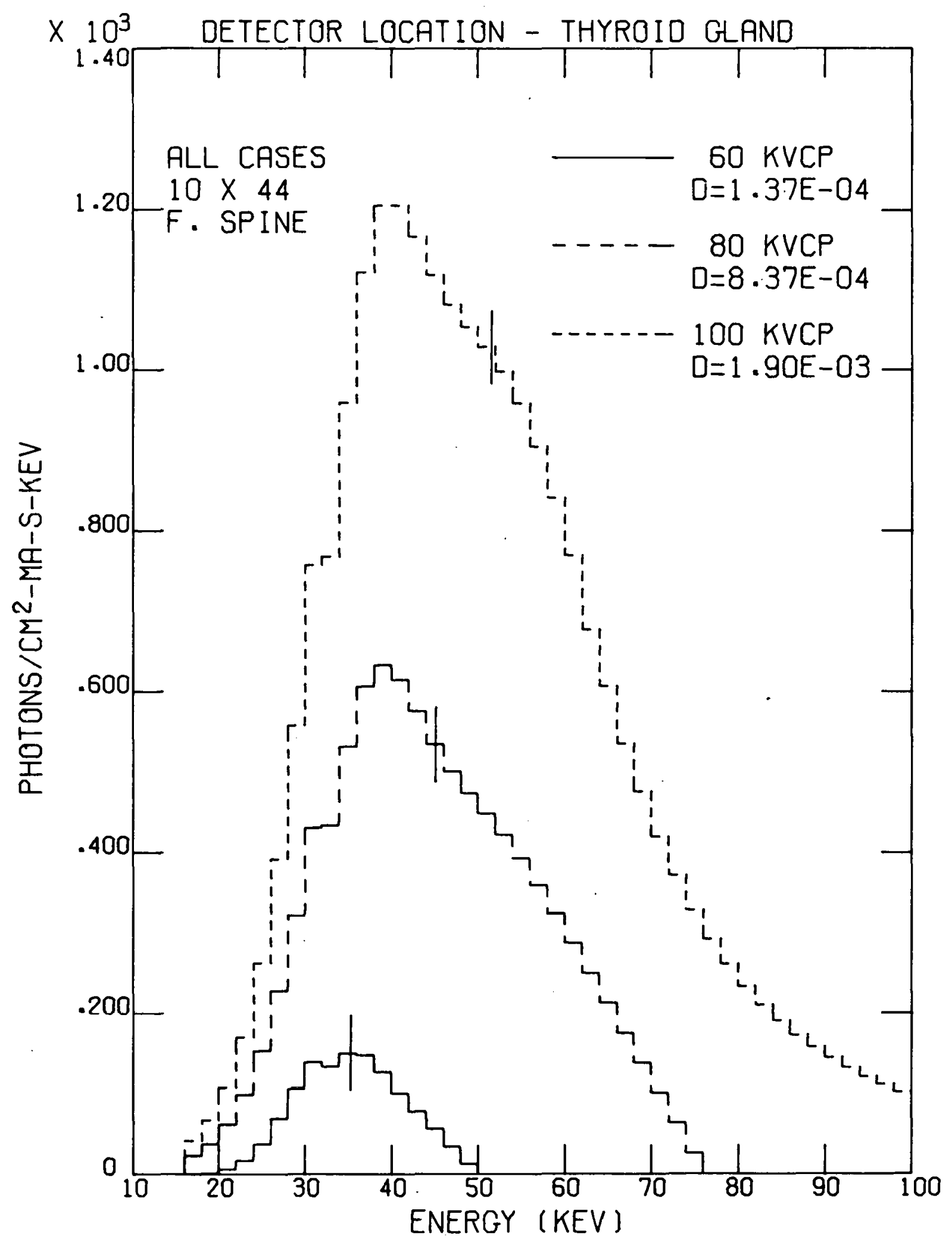

Figure 69. Spectrum at the Thyroid Gland for Full Spine Exam. Vertical ticks indicate mean energy. Field size is in $\mathrm{cm}$. $D$ is the absorbed dose per unit current per unit time $(\mathrm{mrad} / \mathrm{mA}-\mathrm{s})$. 


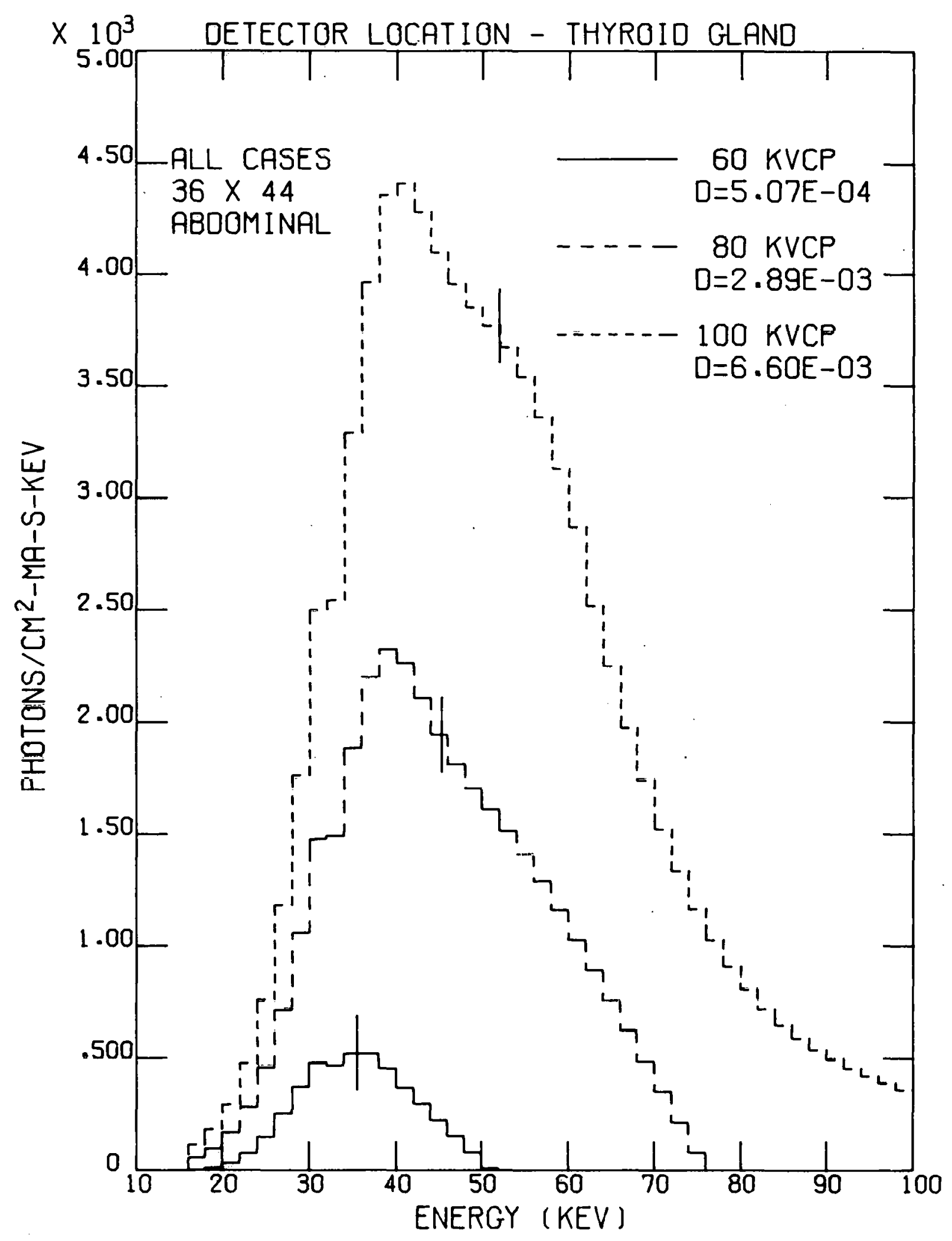

Figure 70. Spectrum at the Thyroid Gland for Abdominal Exam. Vertical ticks indicate mean energy. Field size is in $\mathrm{cm}$. $D$ is the absorbed dose per unit current per unit time (mrad/mA-s). 


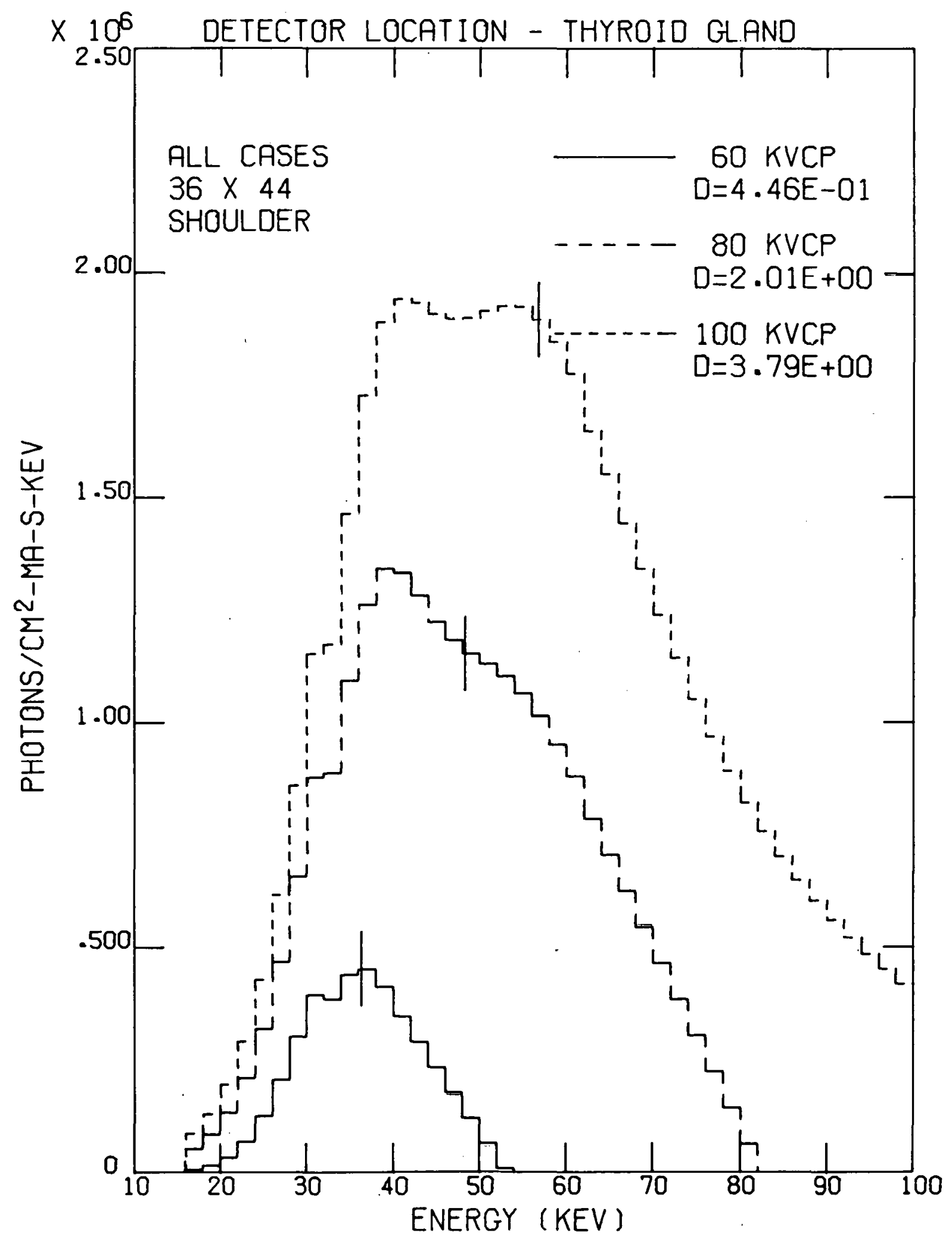

Figure 71. Spectrum at the Thyroid Gland for Shoulder Exam. Vertical ticks indicate mean energy. Field size is in $\mathrm{cm}$. D is the absorbed dose per unit current per unit time (mrad/mA-s). 


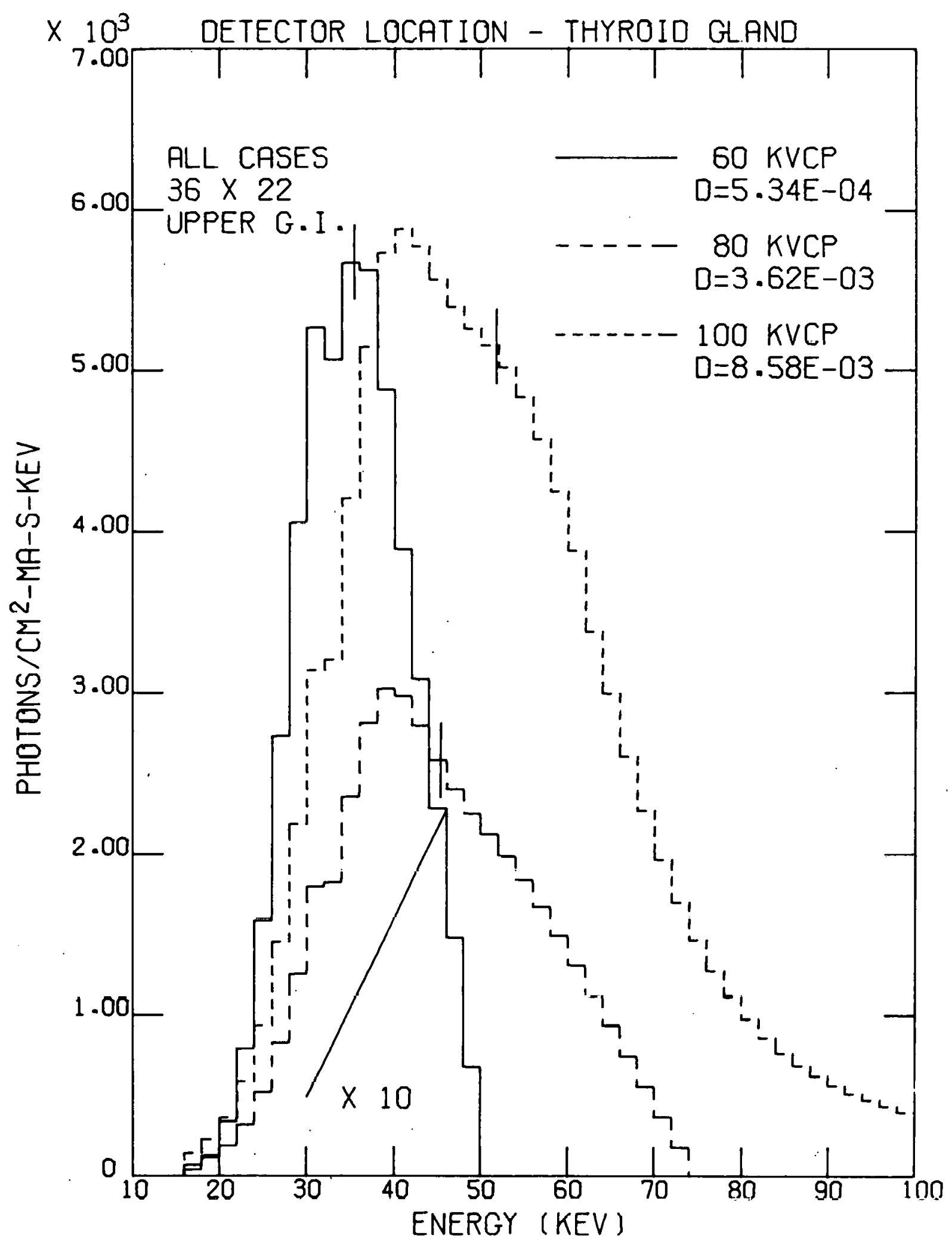

Figure 72. Spectrum at the Thyroid Gland for Upper G.I. Exam. Vertical ticks indicate mean energy. Field size is in $\mathrm{cm}$. $D$ is the absorbed dose per unit current per unit time (mrad/mA-s). 


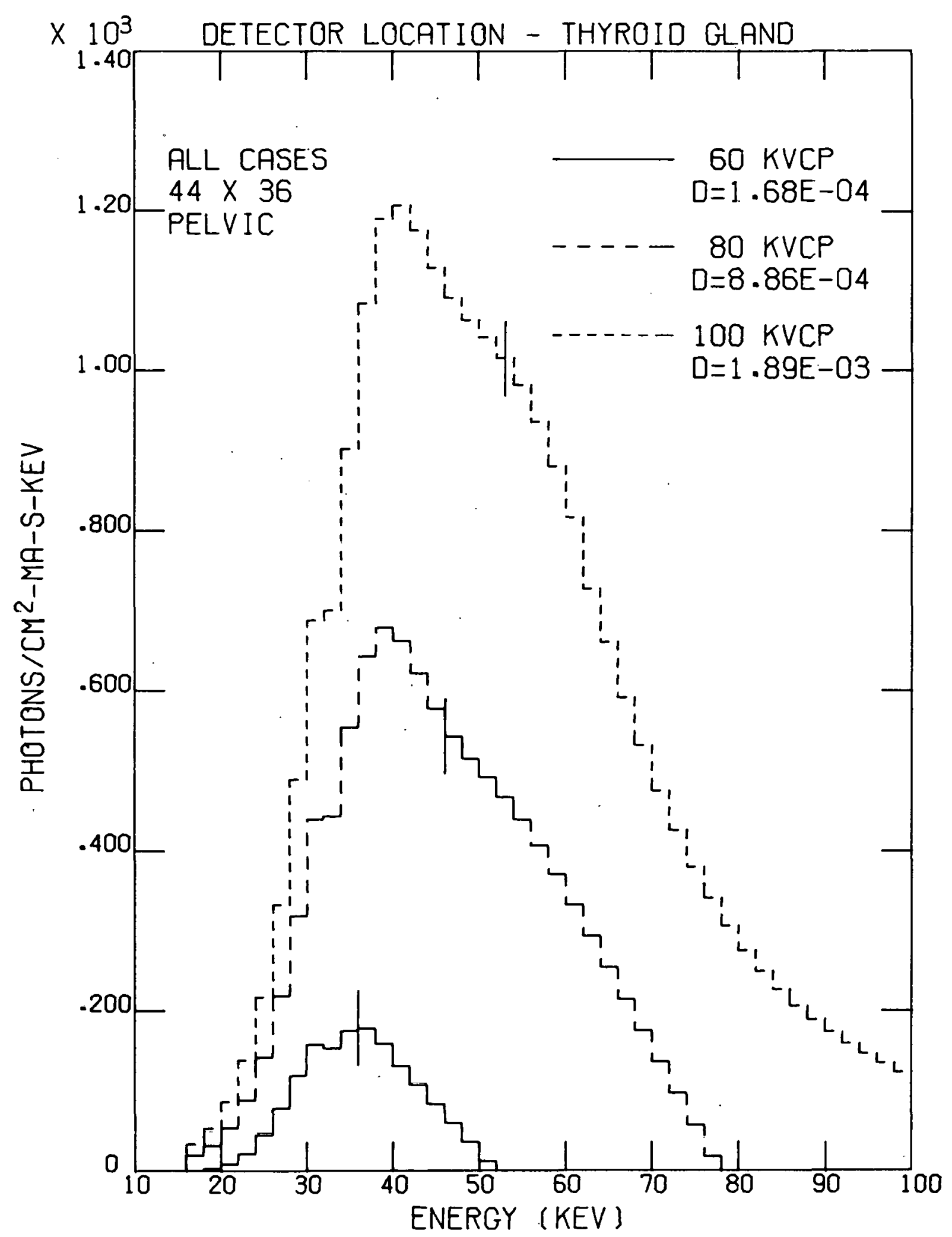

Figure 73. Spectrum at the Thyroid Gland for Pelvic Exam. Vertical ticks indicate mean energy. Field size is in $\mathrm{cm}$. D is the abosrbed dose per unit current per unit time (mrad/mA-s). 


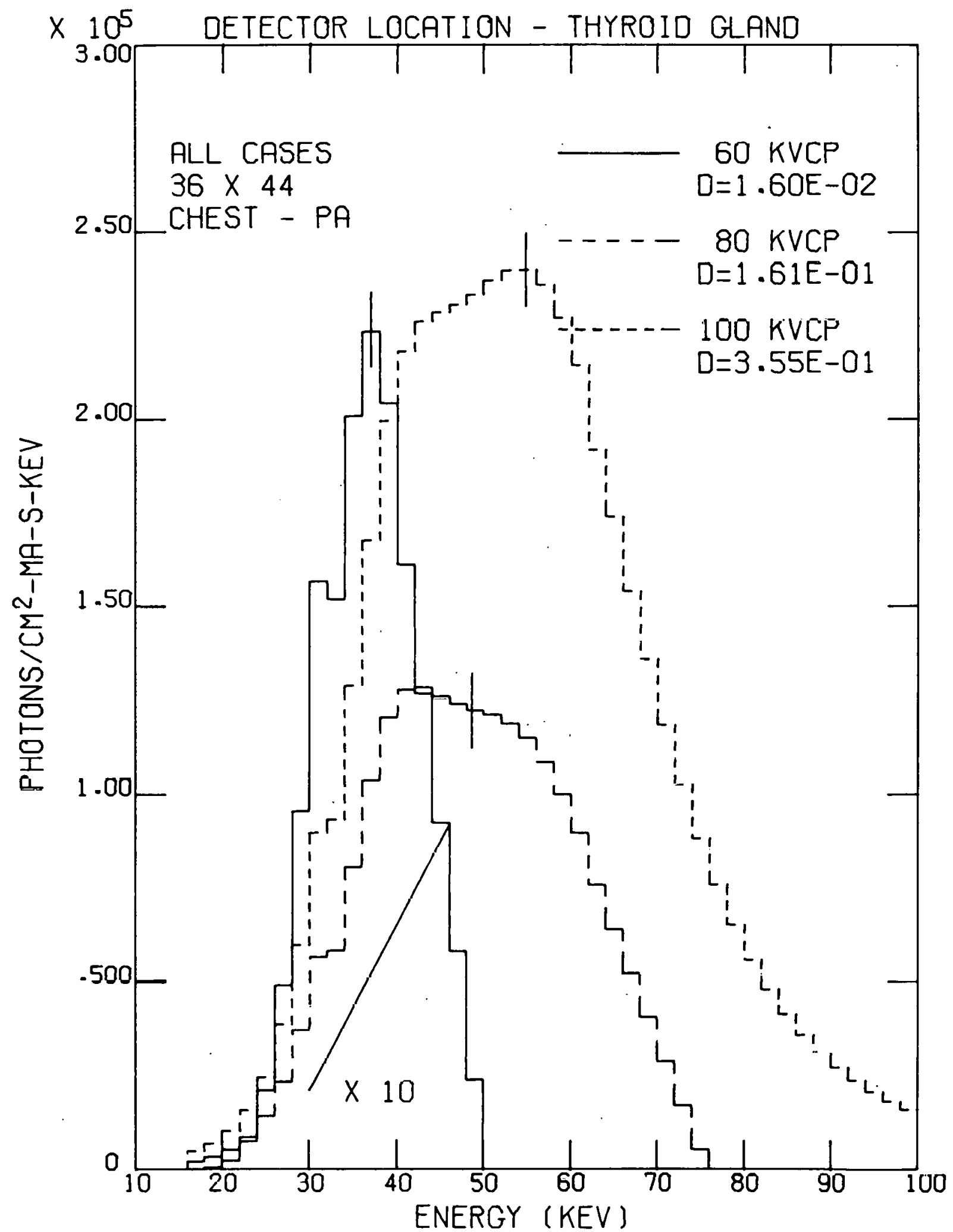

Figure 74. Spectrum at the Thyroid Gland for Chest Exam. Vertical ticks indicate mean energy. Field size is in $\mathrm{cm}$. $D$ is the absorbed dose per unit current per unit time (mrad/mA-s). 


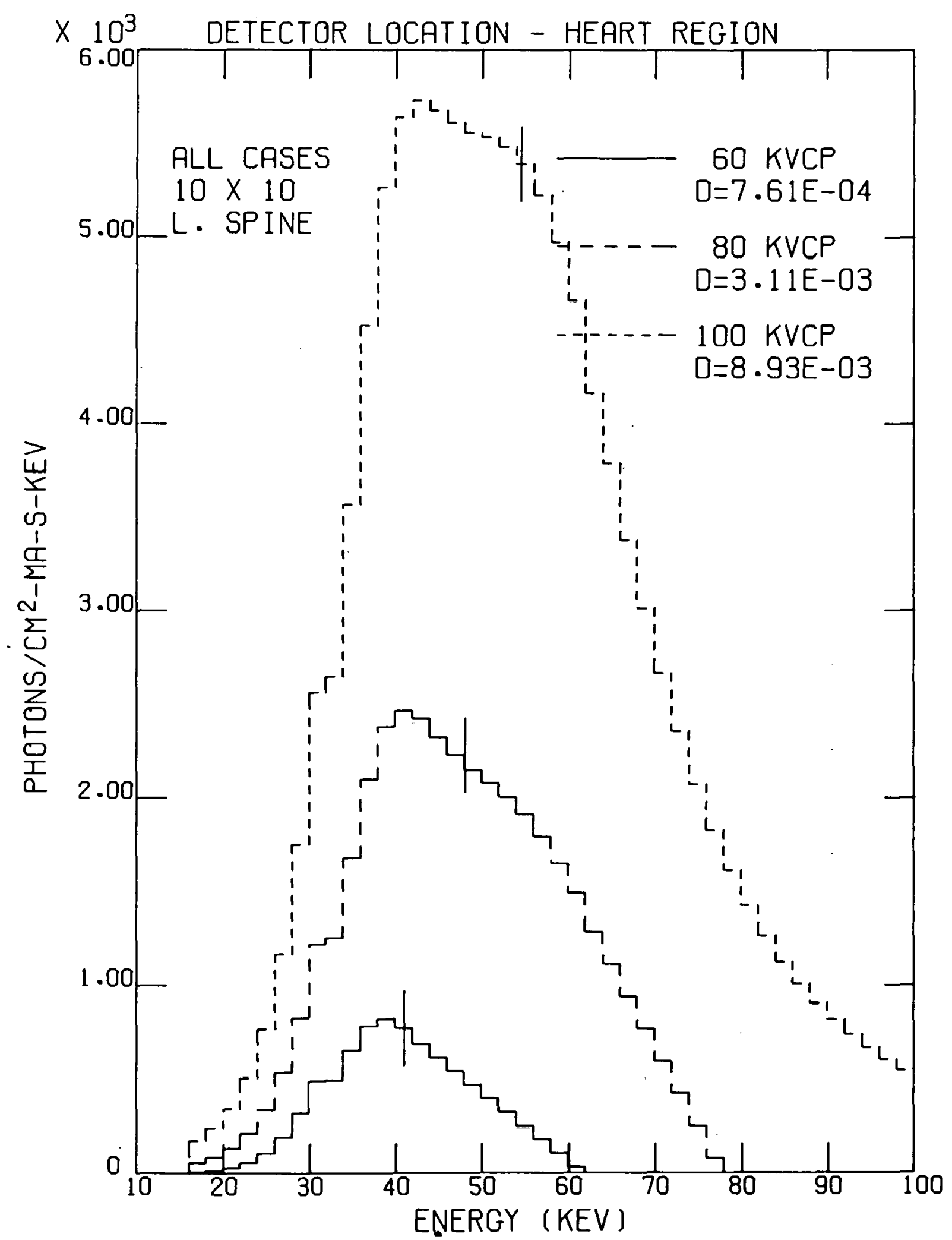

Figure 75. Spectrum at the Heart Region for Lower Spine Exam. Vertical ticks indicate mean energy. Field size is in $\mathrm{cm}$. $D$ is the absorbed dose per unit current per unit time (mrad/mA-s). 


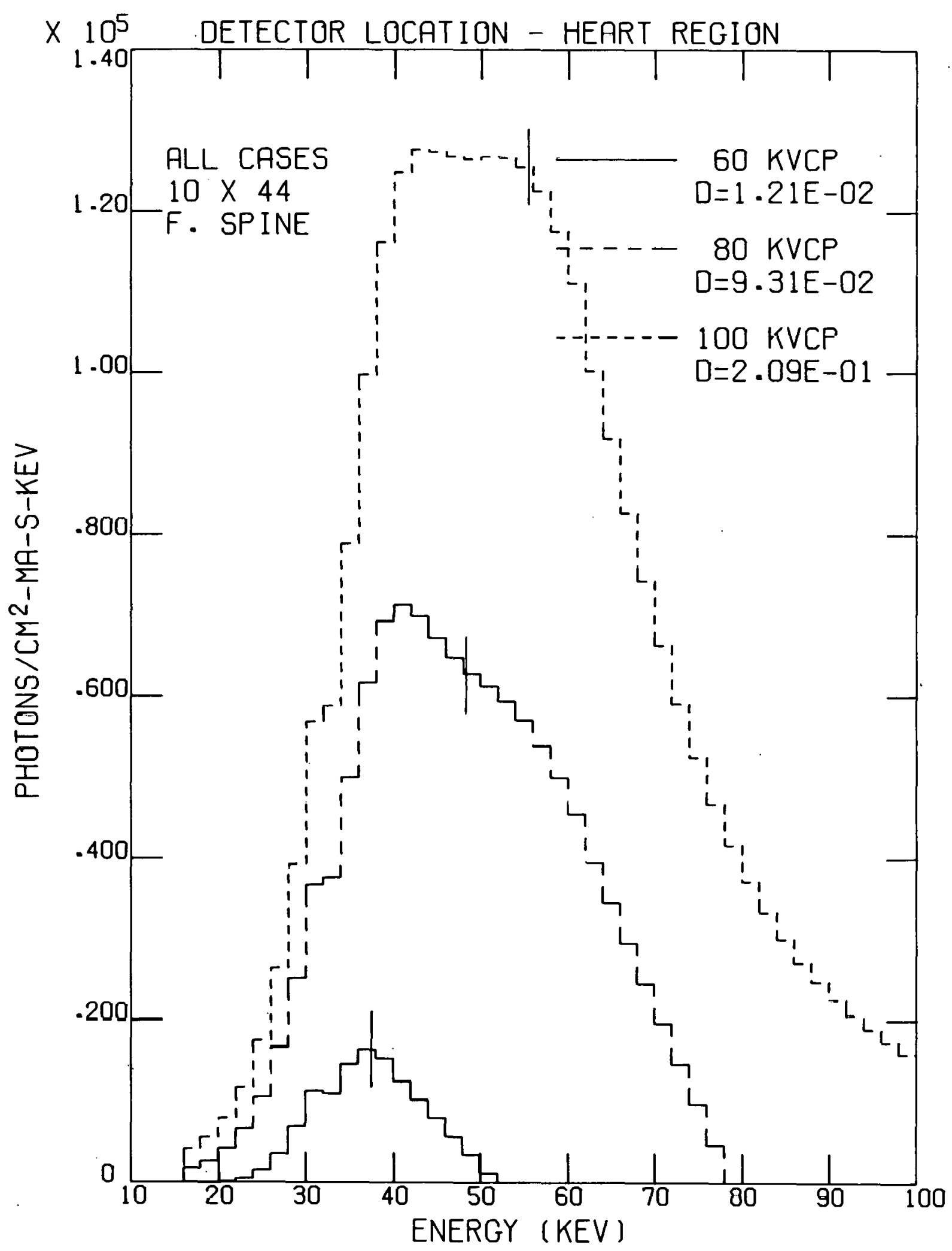

Figure 76. Spectrum at the Heart Region for Full Spine Exam. Vertical ticks indicate mean energy. Field size is in $\mathrm{cm}$. D is the absorbed dose per unit current per unit time (mrad/mA-s). 


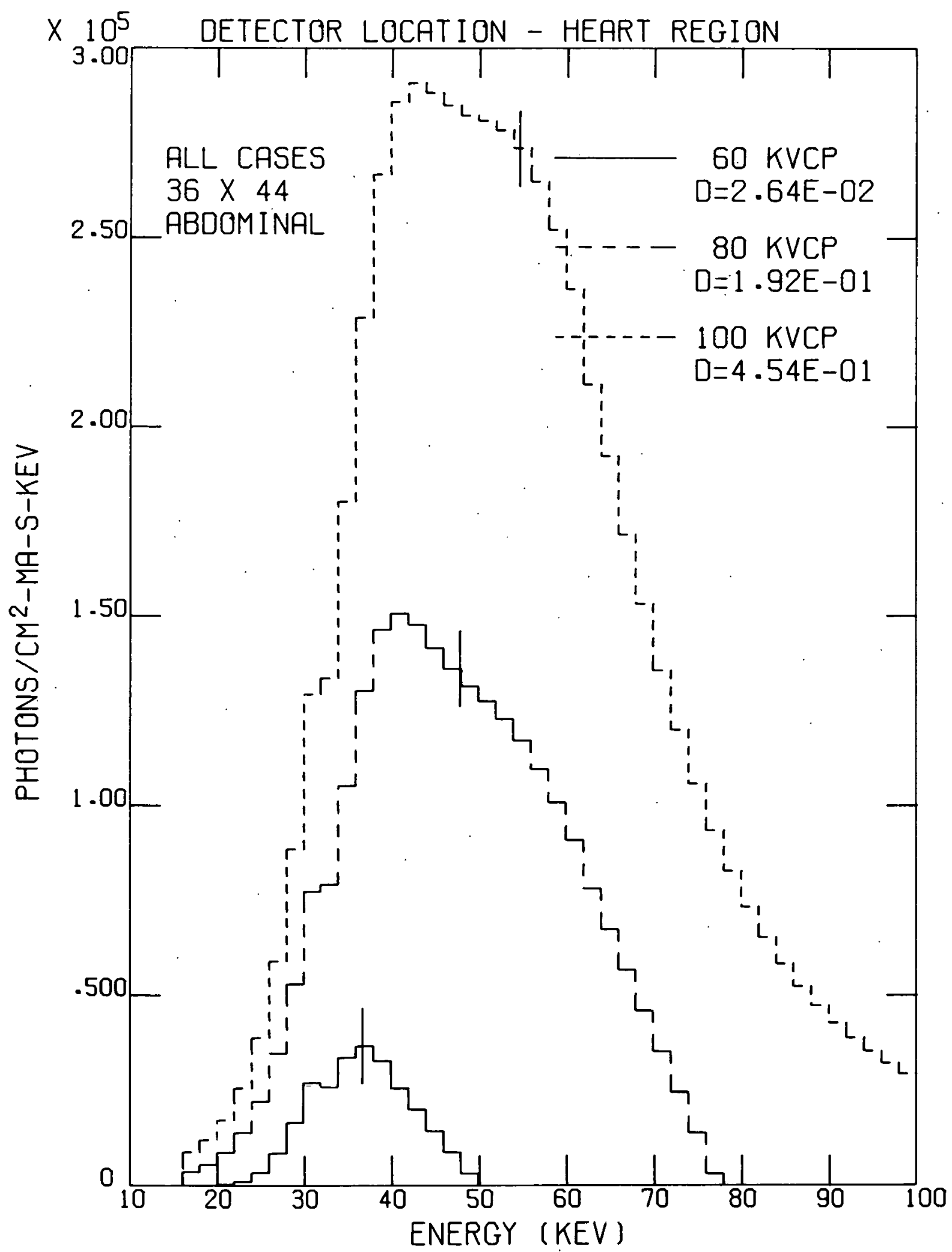

Figure 77. Spectrum at the Heart Region for Abdominal Exam. Vertical ticks indicate mean energy. Field size is in $\mathrm{cm}$. D is the absorbed dose per unit current per unit time (mrad/mA-s). 


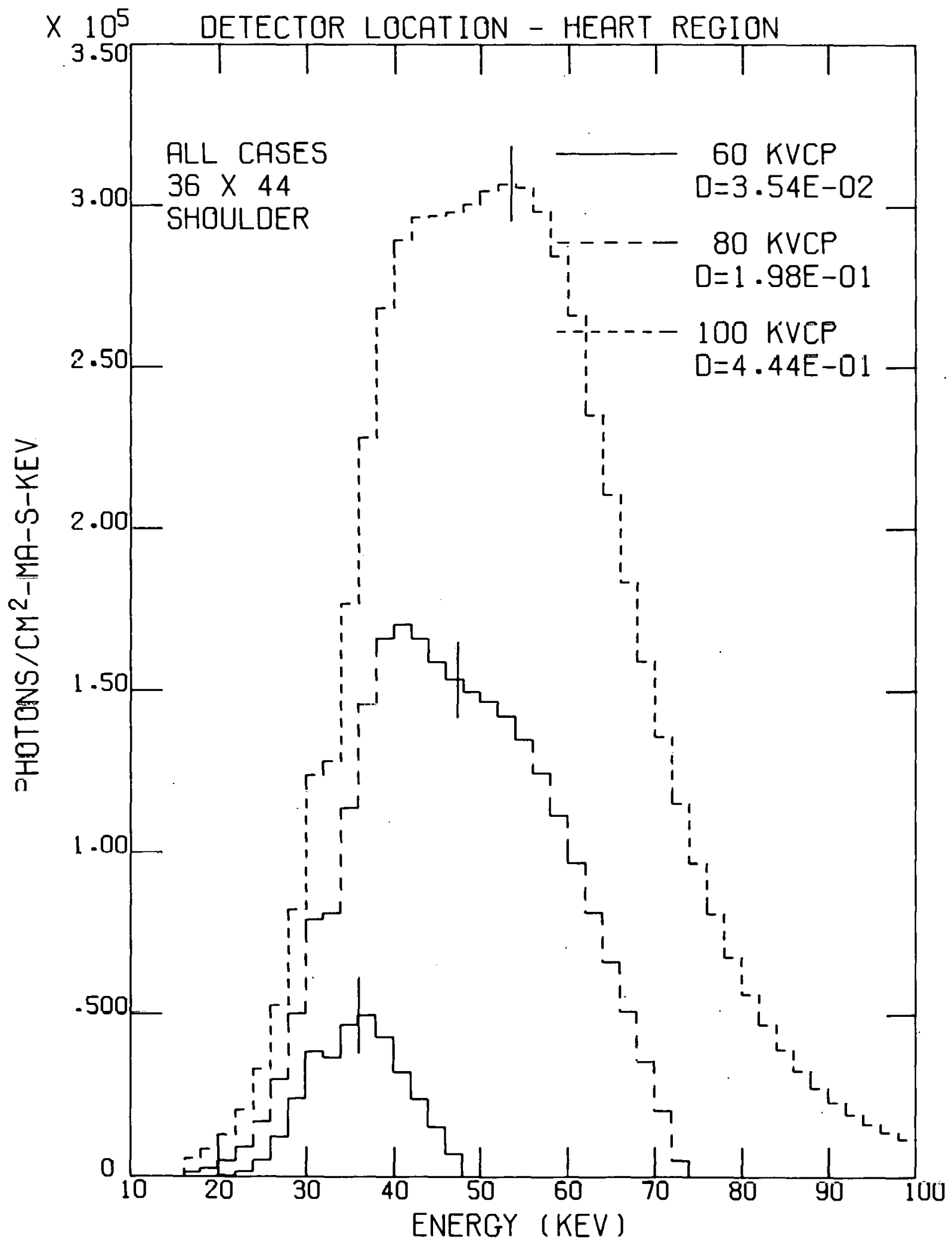

Figure 78. Spectrum at the Heart Region for Shoulder Exam. Vertical ticks indicate mean energy. Field size is in $\mathrm{cm}$. $D$ is the absorbed dose per unit current per unit time (mrad/mA-s). 


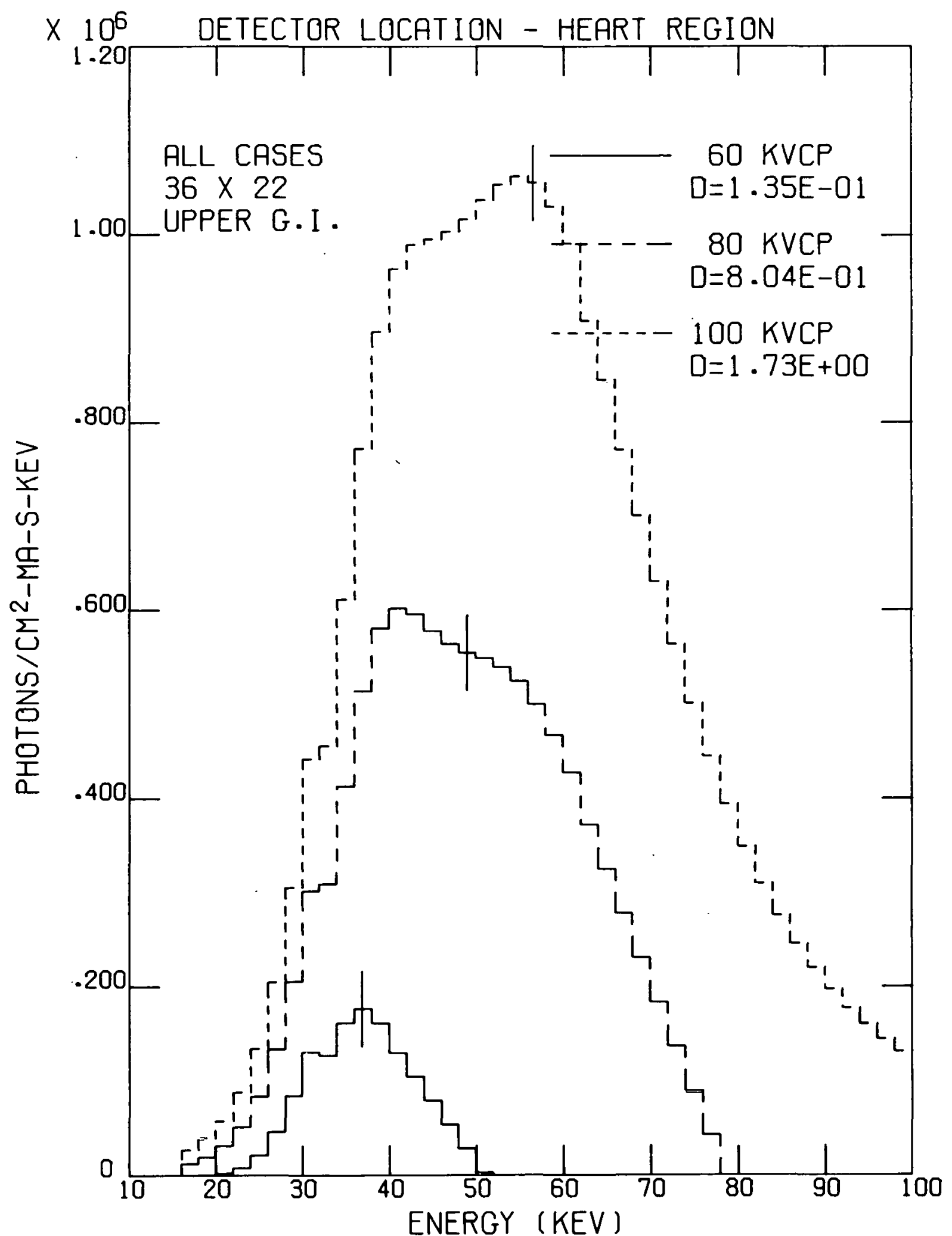

Figure 79. Spectrum at the Heart Region for Upper G.I. Exam. Vertical ticks indicate mean energy. Field size is in $\mathrm{cm}$. $D$ is the absorbed dose per unit current per unit time (mrad/mA-s). 


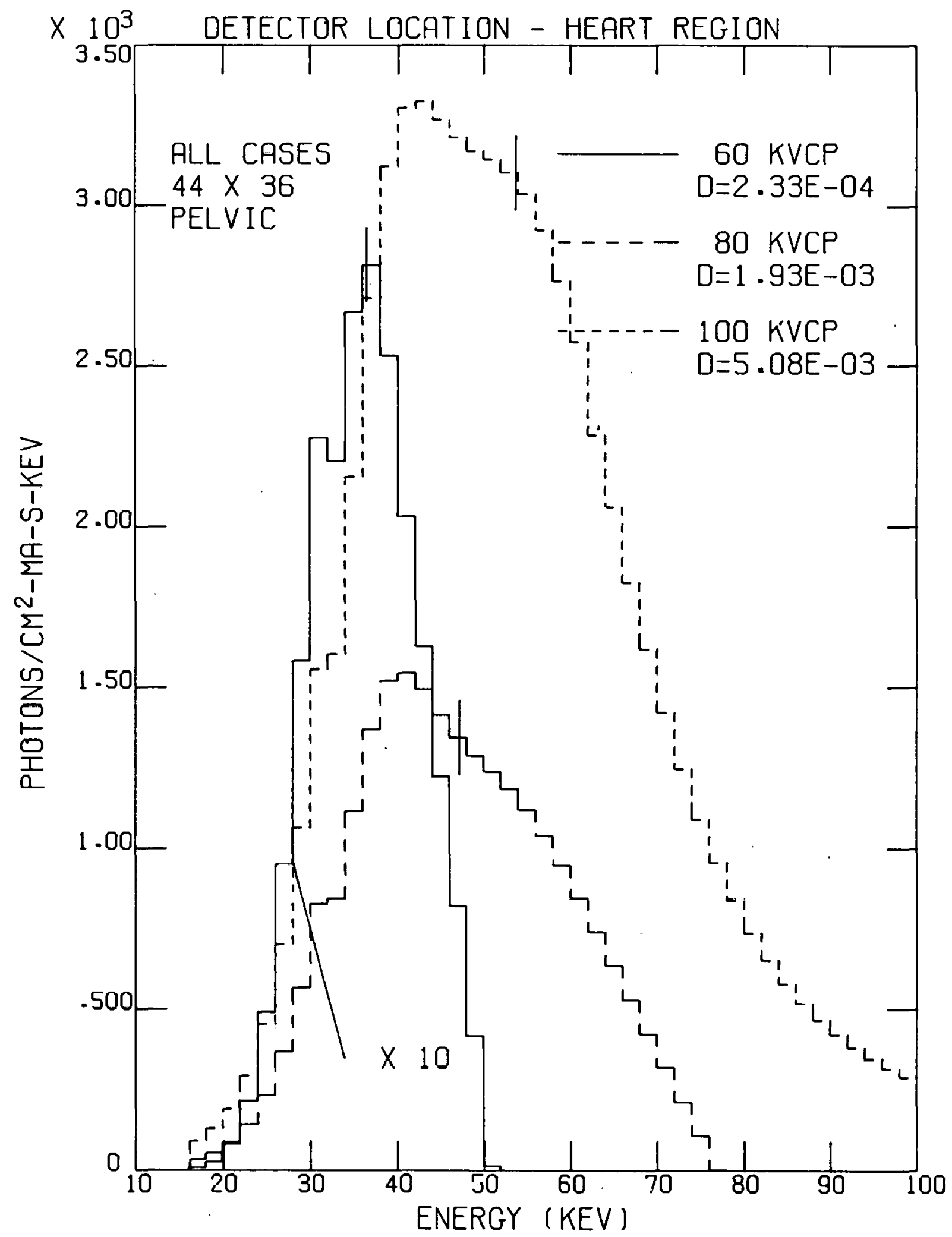

Figure 80. Spectrum at the Heart Region for Pelvic Exam. Vertical ticks indicate mean energy. Field size is in $\mathrm{cm}$. $D$ is the absorbed dose per unit current per unit time (mrad/mA-s). 


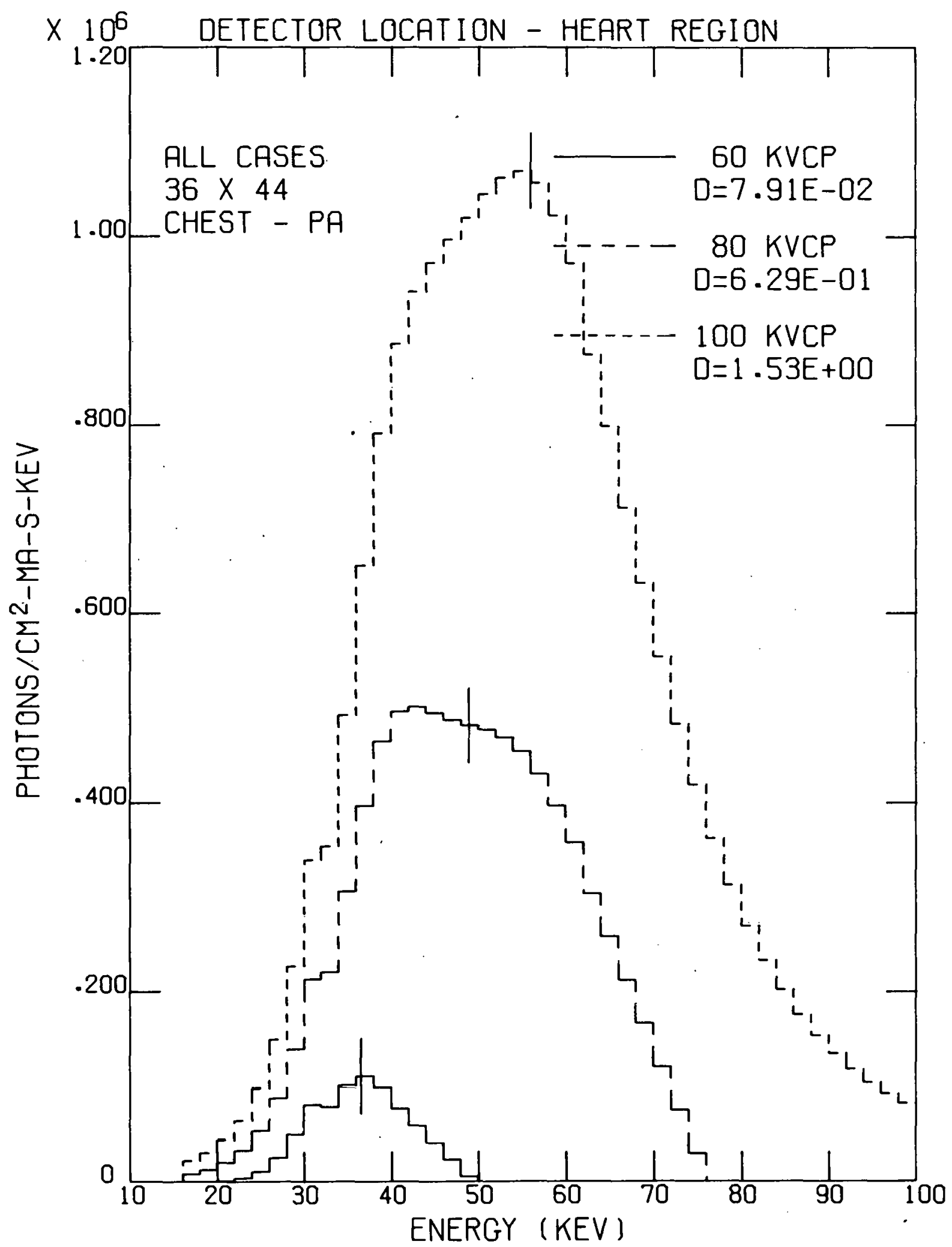

Figure 81. Spectrum at the Heart Region for Chest Exam. Vertical ticks indicate mean energy. Field size is in $\mathrm{cm}$. $D$ is the absorbed dose per unit current per unit time $(\mathrm{mrad} / \mathrm{mA}-\mathrm{s})$. 


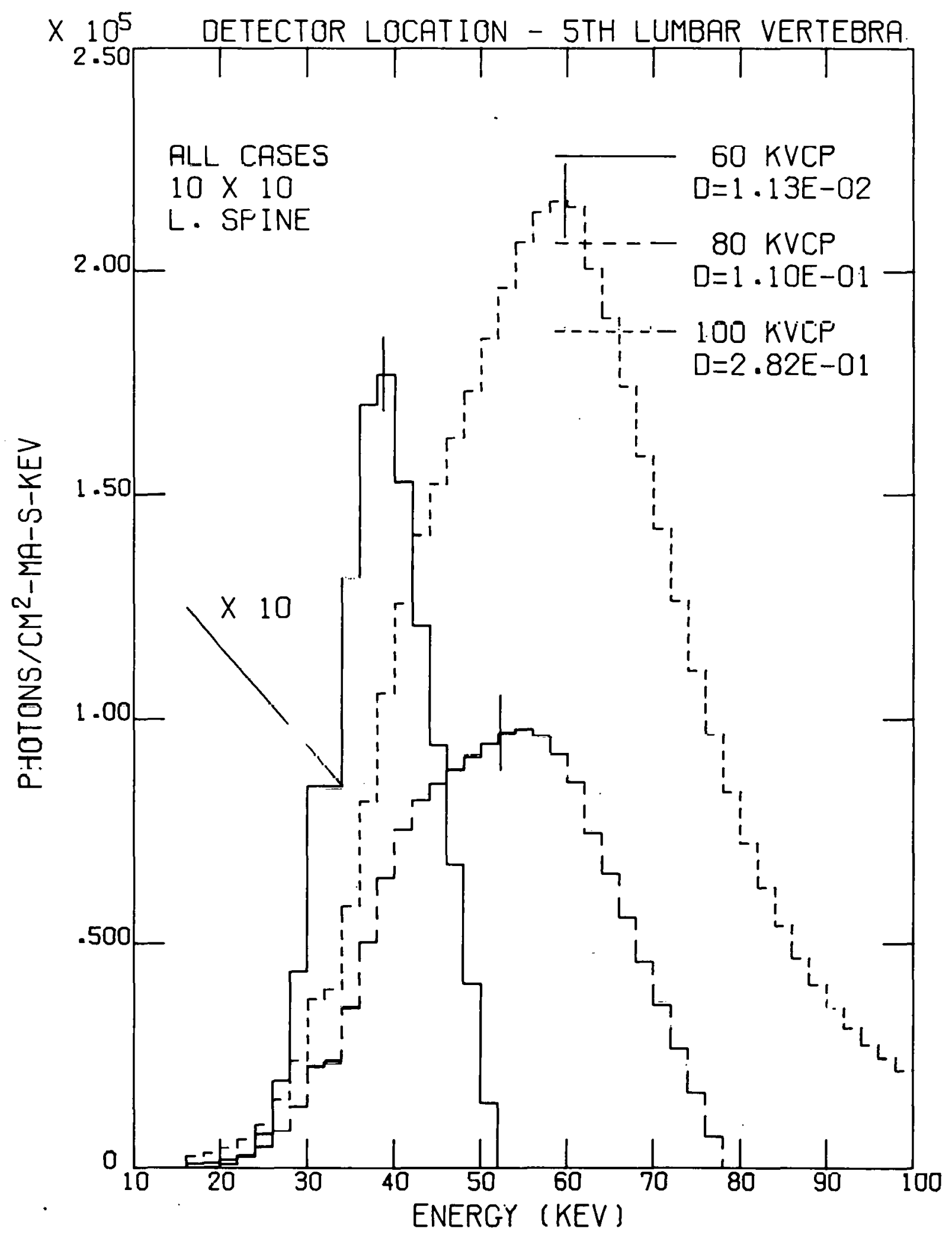

Figure 82. Spectrum at the Fifth Lumbar Vertebra for Lower Spine Exam. Vertical ticks indicate mean energy. Field size is in $\mathrm{cm}$. $D$ is the absorbed dose per unit current per unit time (mrad/mA-s). 


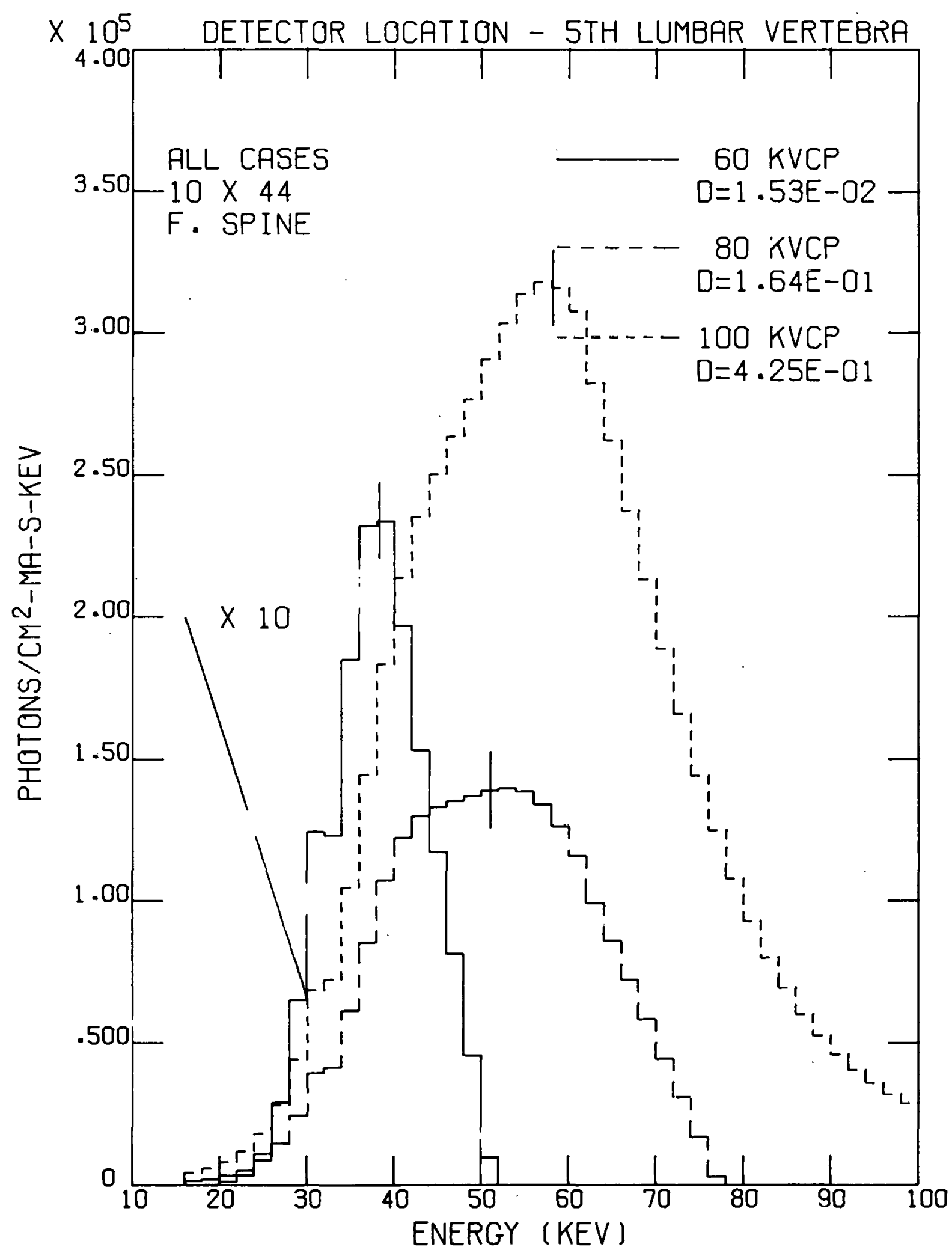

Figure 83. Spectrum at the Fifth Lumbar Vertebra for Full Spine Exam. Vertical ticks indicate mean energy. Field size is in $\mathrm{cm}$. $D$ is the absorbed dose per unit current per unit time (mrad/mA-s). 


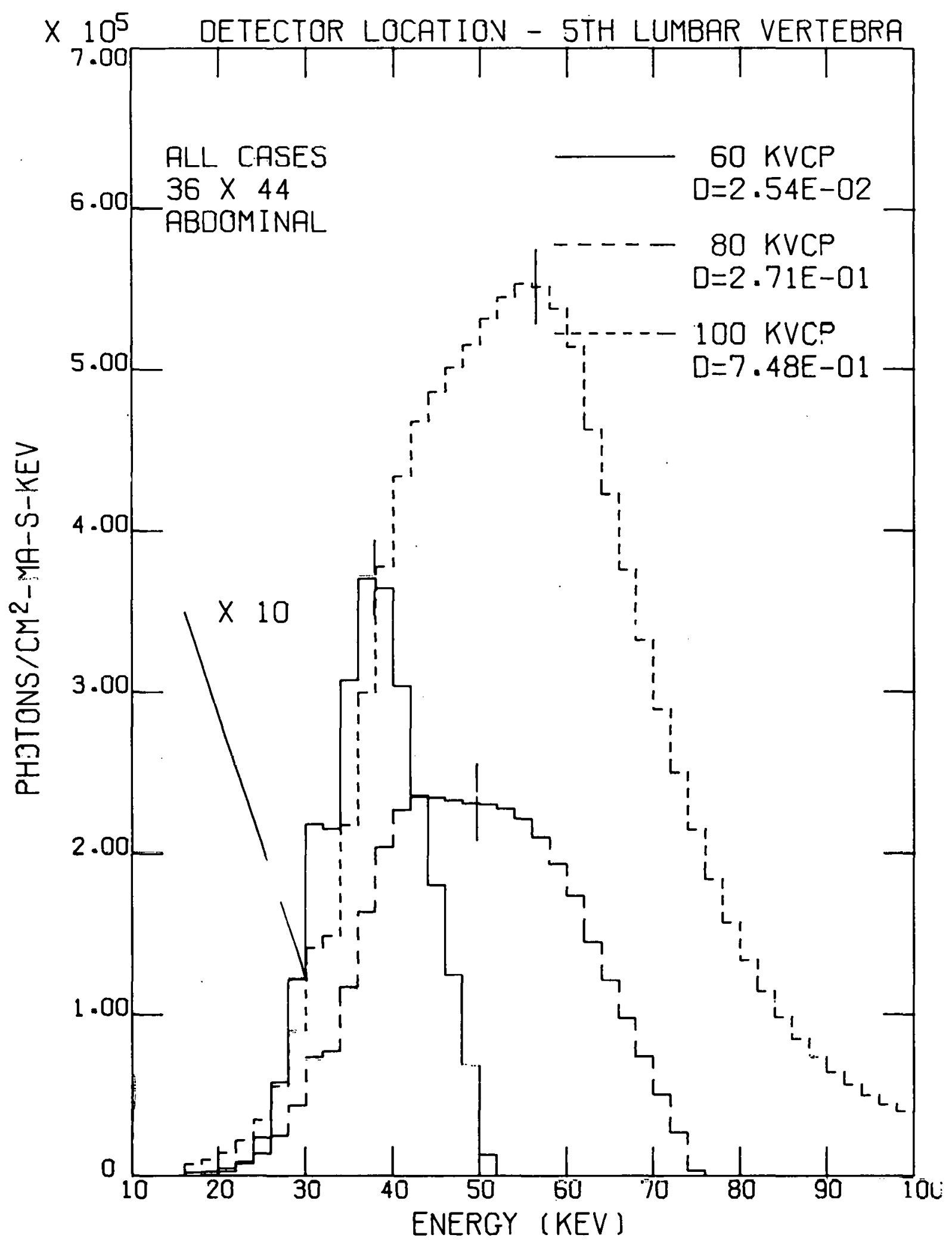

Figure 84. Spectrum at the Fifth Lumbar Vertebra for Abdominal Exam. Vertical ticks indicate mean energy. Field size is in $\mathrm{cm}$. $D$ is the absorbed dose per unit current per unit time (mrad/mA-s). 


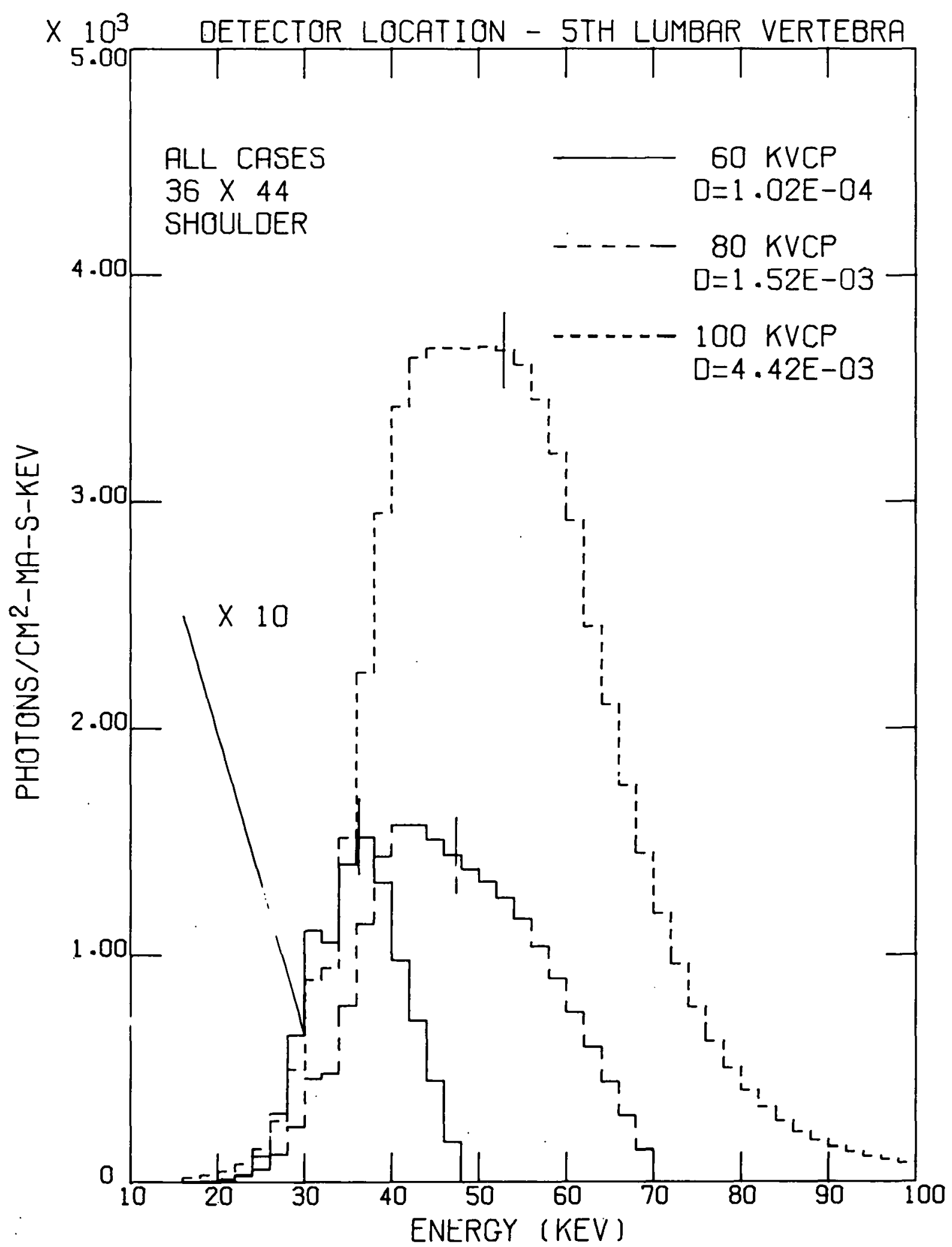

Figure 85. Spectrum at the Fifth Lumbar Vertebra for Shoulder Exam. Vertical ticks indicate mean energy. Field size is in $\mathrm{cm}$. $D$ is the absorbed dose per unit current per unit time (mrad/mA-s). 


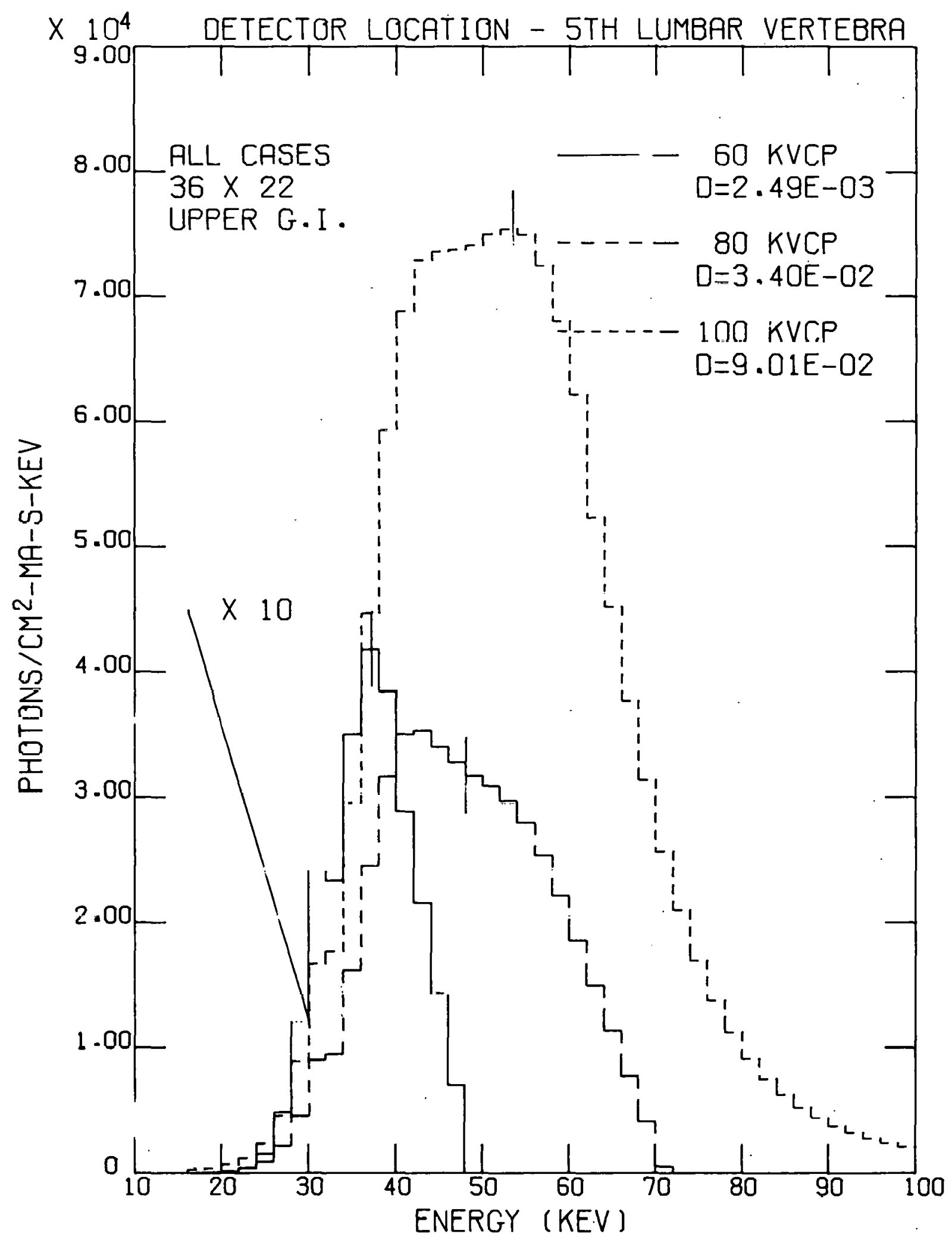

Figure 86. Spectrum at the Fifth Lumbar Vertebra for Upper G.I. Exam. Vertical ticks indicate mean energy. Field size is in $\mathrm{cm}$. $D$ is the absorbed dose per unit current per unit time $(\mathrm{mrad} / \mathrm{mA}-\mathrm{s})$. 


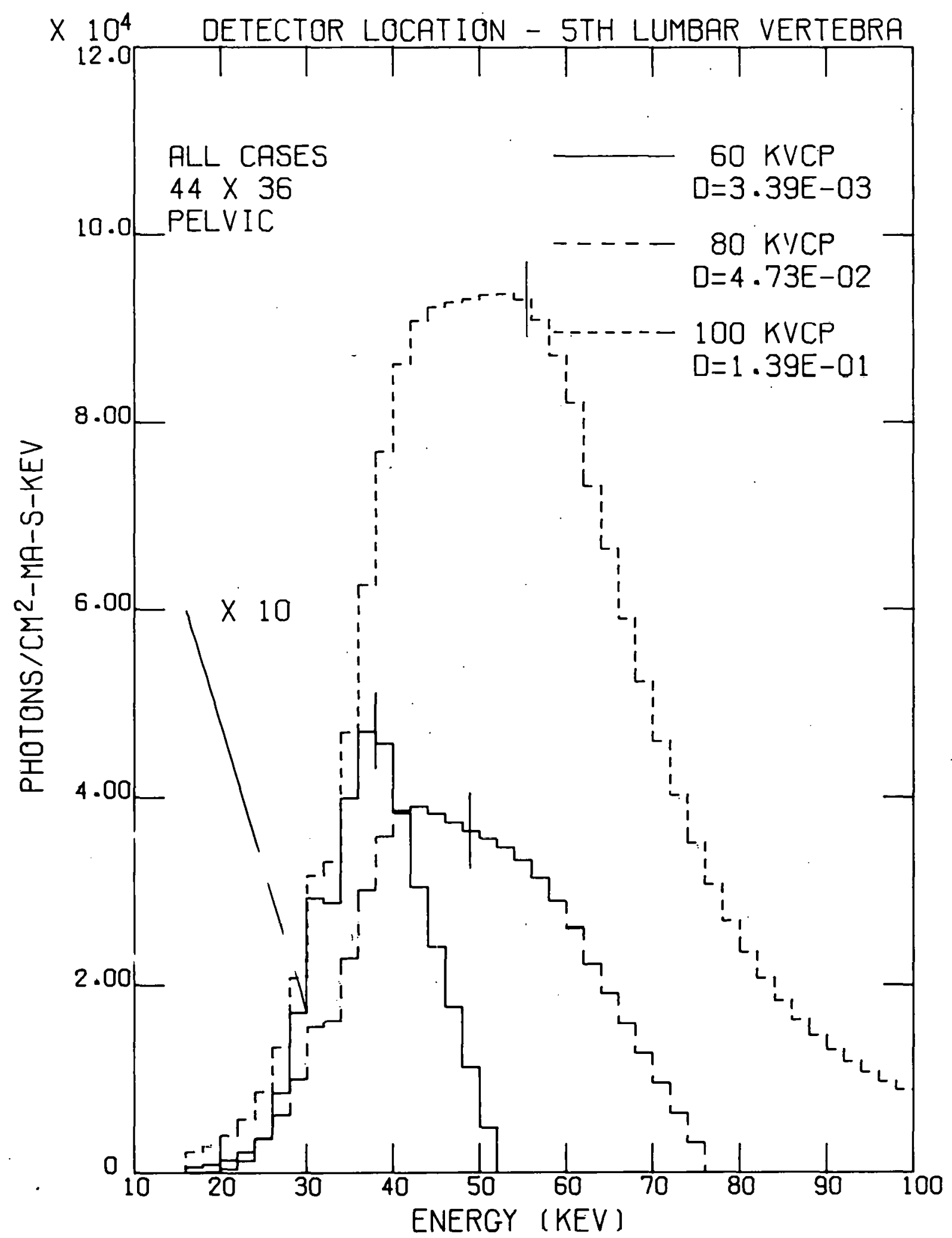

Figure 87. Spectrum at the Fifth Lumbar Vertebra for Pelvic Exam. Vertical ticks indicate mean energy. Field size is in $\mathrm{cm}$. $D$ is the absorbed dose per unit current per unit time (mrad/mA-s). 


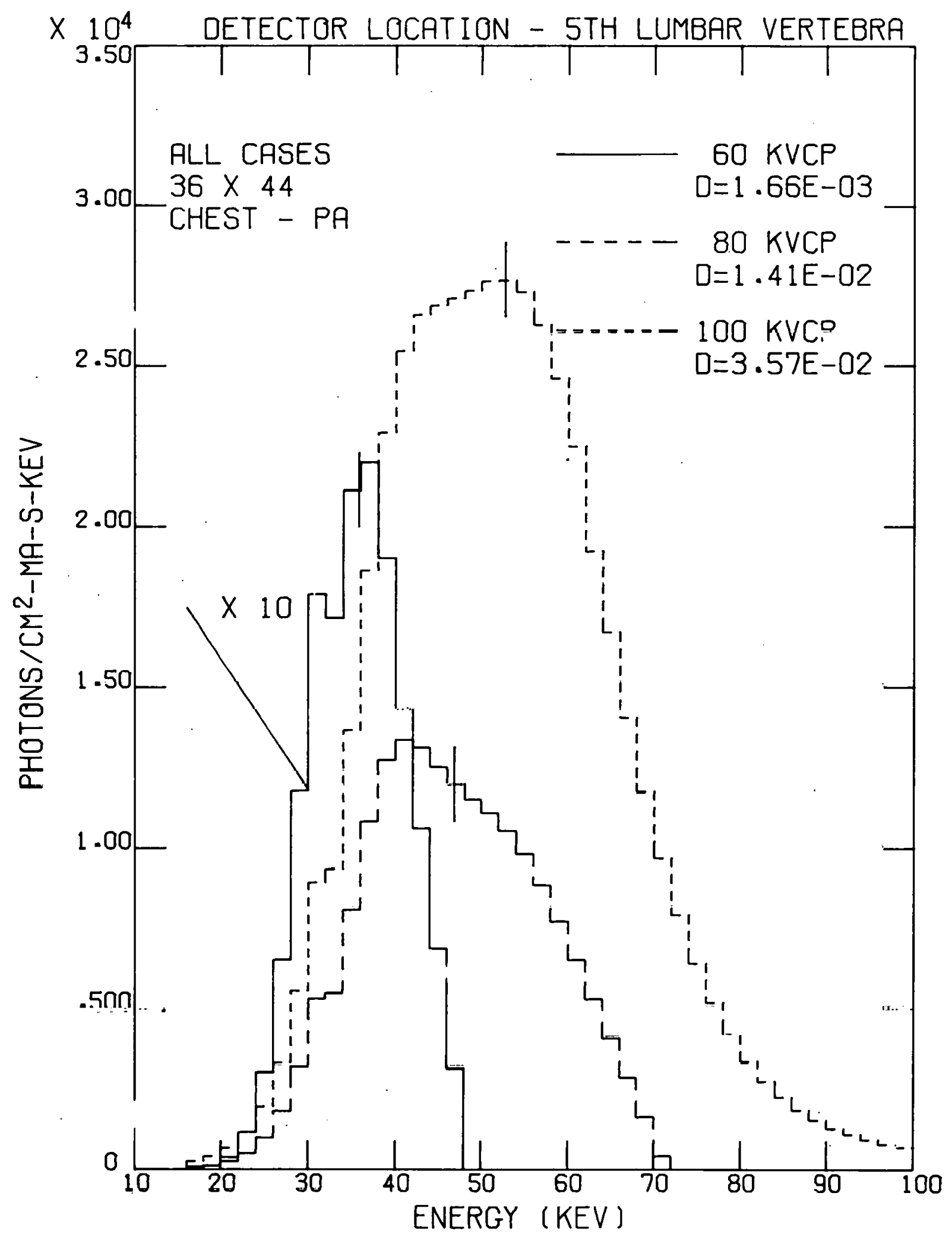

Figure 88. Spectrum at the Fifth Lumbar Vertebra for Chest Exam. Vertical ticks indicate mean energy. Field size is in $\mathrm{cm}$. $D$ is the absorbed dose per unit current per unit time (mrad/mA-s). 


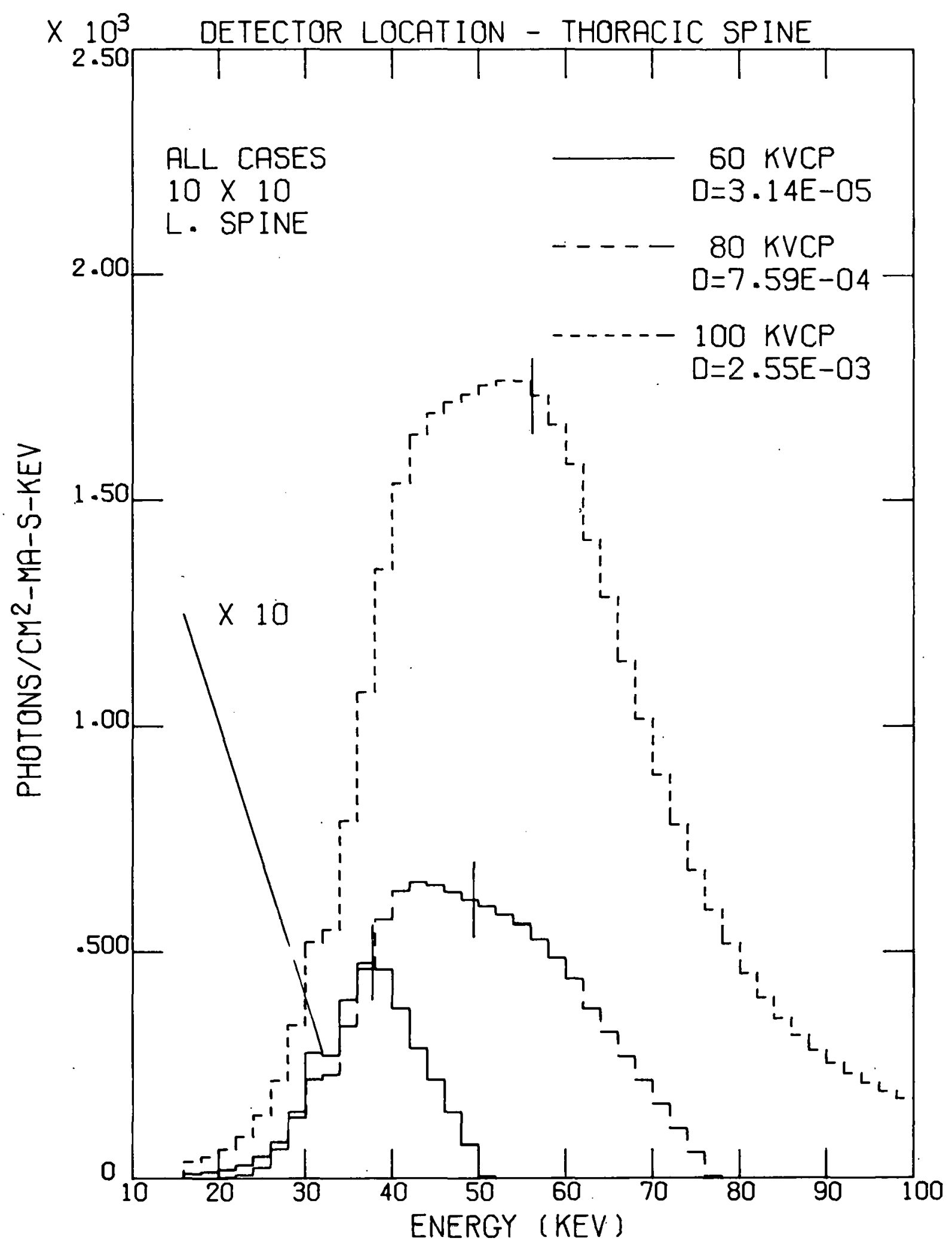

Figure 89. Spectrum at the Thoracic Spine for Lower Spine Exam. Vertical ticks indicate mean energy. Fleld size is in $\mathrm{cm}$. $D$ is the absorbed dose per unit current per unit time (mrad/mA-s). 


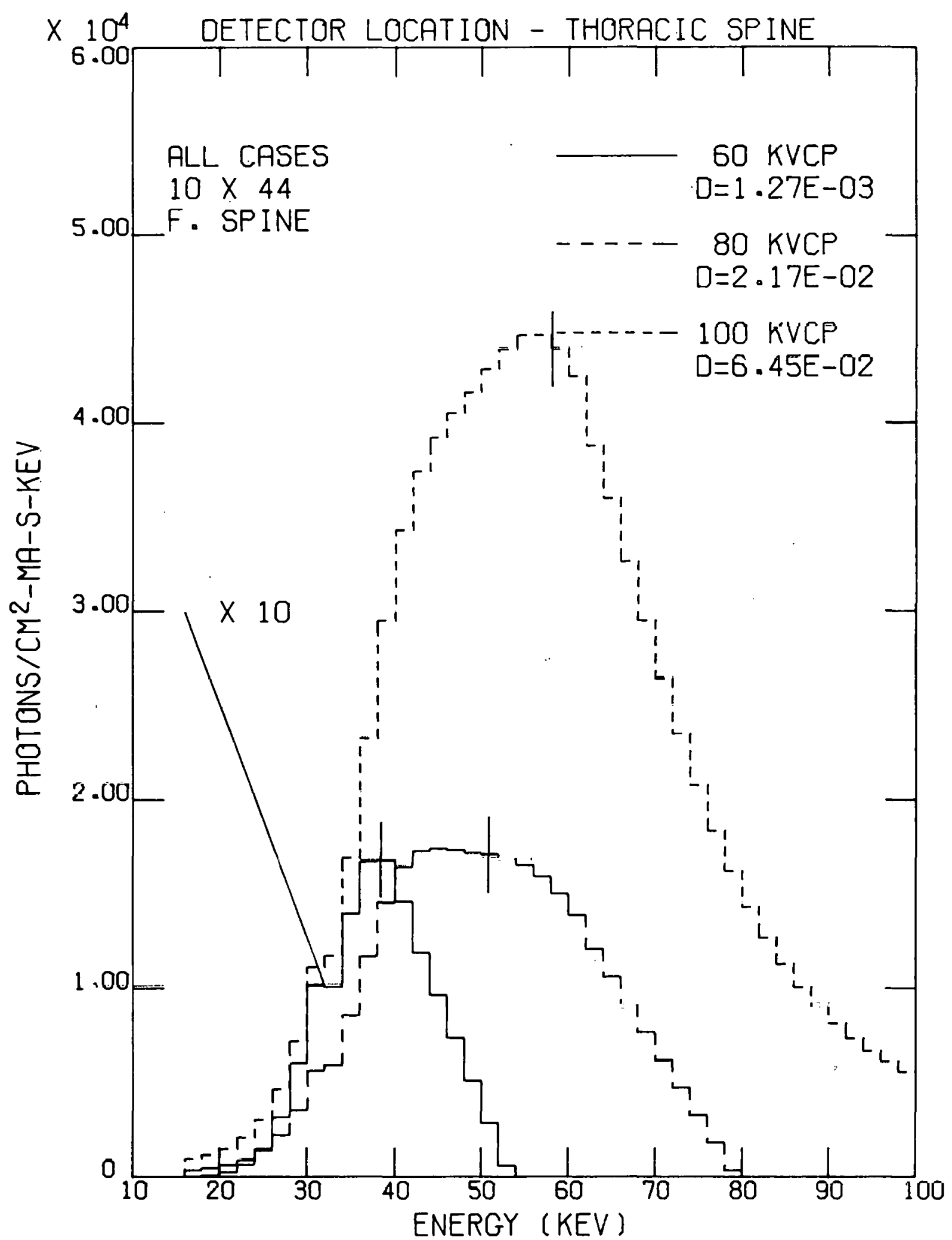

Figure 90. Spectrum at the Thoracic Spine for Full Spine Exam. Vertical ticks indicate mean energy. Field size is in $\mathrm{cm}$. $D$ is the absorbed dose per unit current per unit time (mrad/mA-s). 


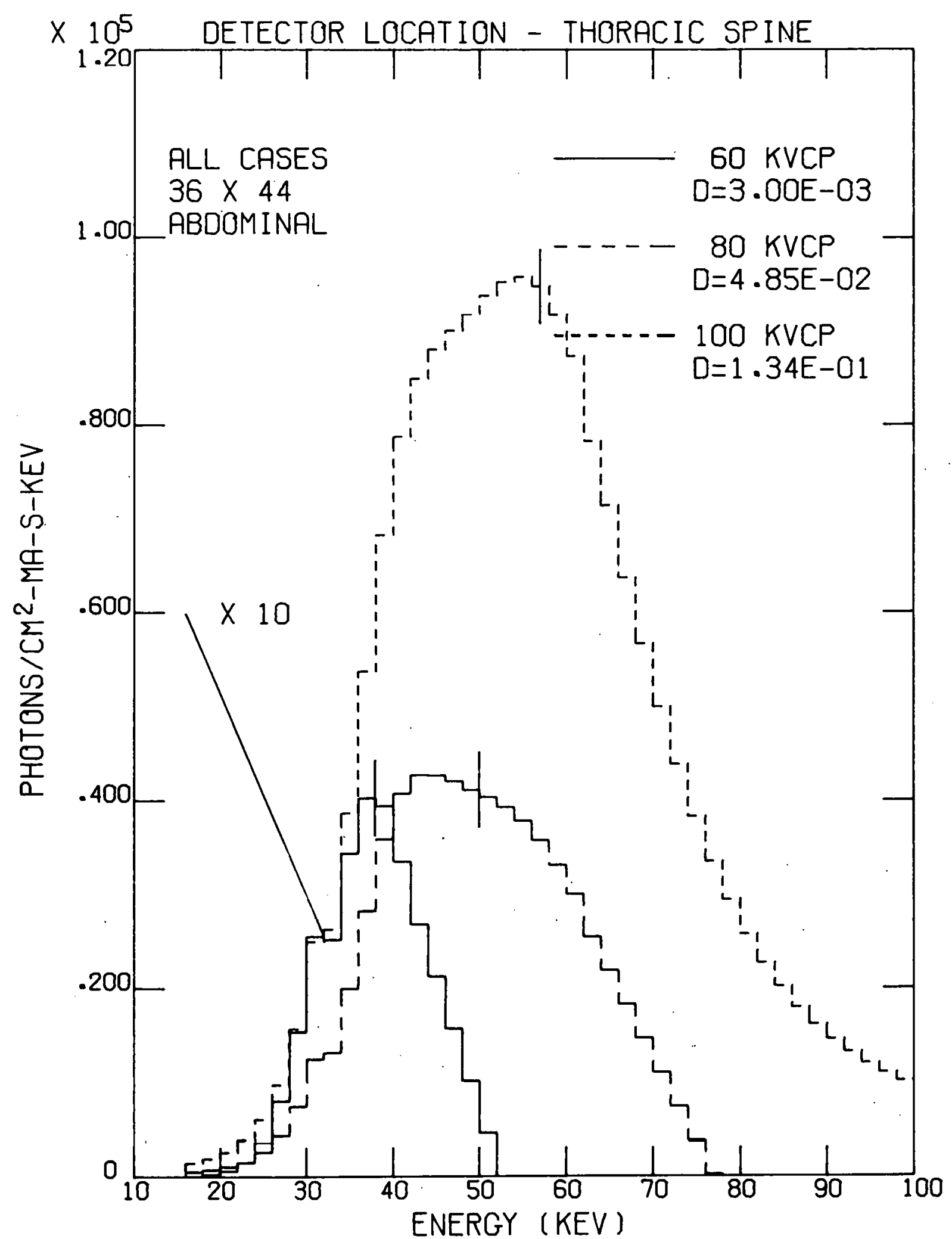

Figure 91. Spectrum at the Thoracic Spine for Abdominal Exam. Vertical ticks indicate mean energy. Field size is in $\mathrm{cm}$. $D$ is the absorbed dose per unit current per unit time (mrad/mA-s). 


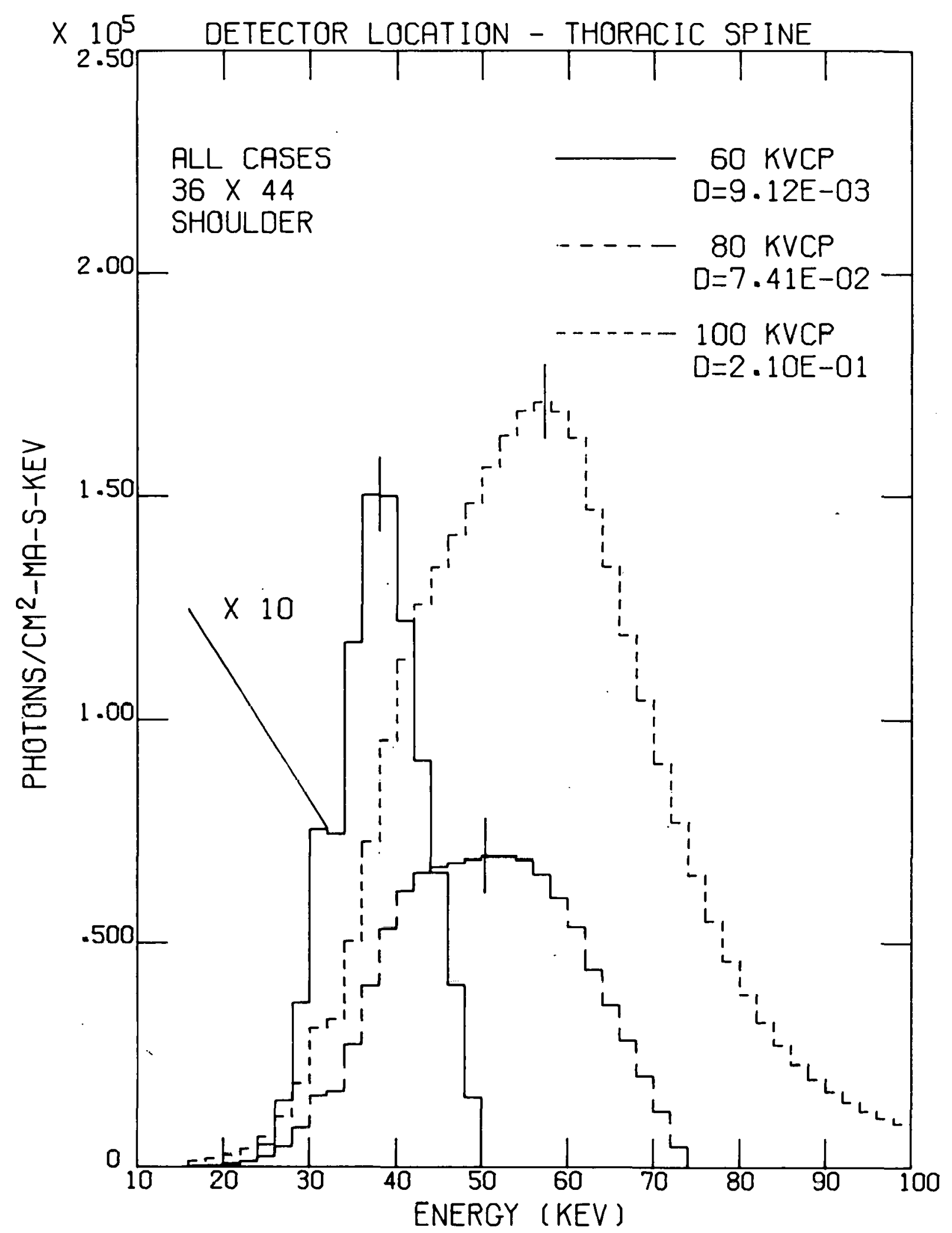

Figure 92. Spectrum at the Thoracic Spine for Shoulder Exam.

Vertical ticks indicate mean energy. Field size is in $\mathrm{cm}$. $D$ is the absorbed dose per unit current per unit time (mrad/mA-s). 


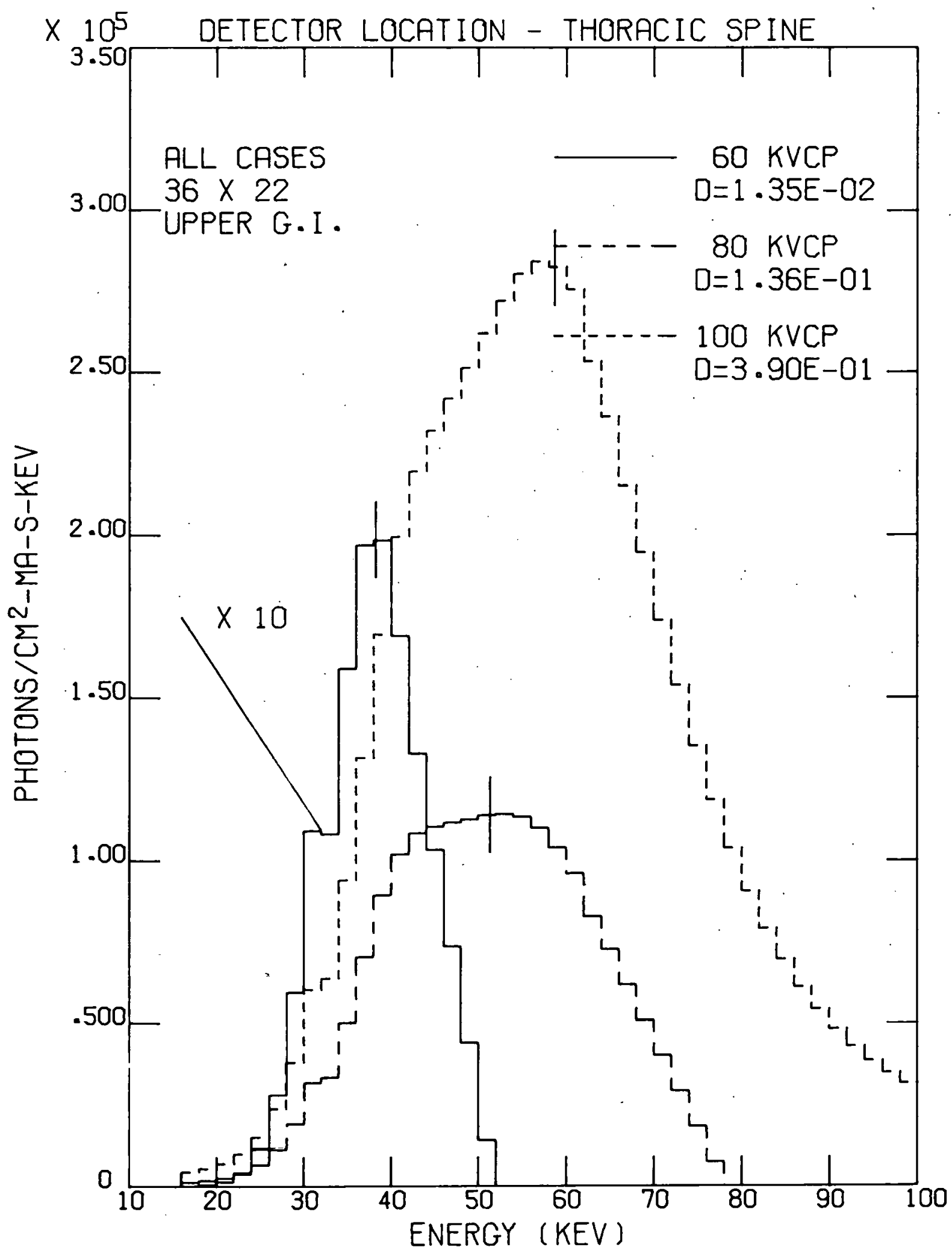

Figure 93. Spectrum at the Thoracic Spine for Upper G.I. Exam. Vertical ticks indicate mean energy. Field size is in $\mathrm{cm}$. $D$ is the absorbed dose per unit current per unit time (mrad/mA-s). 


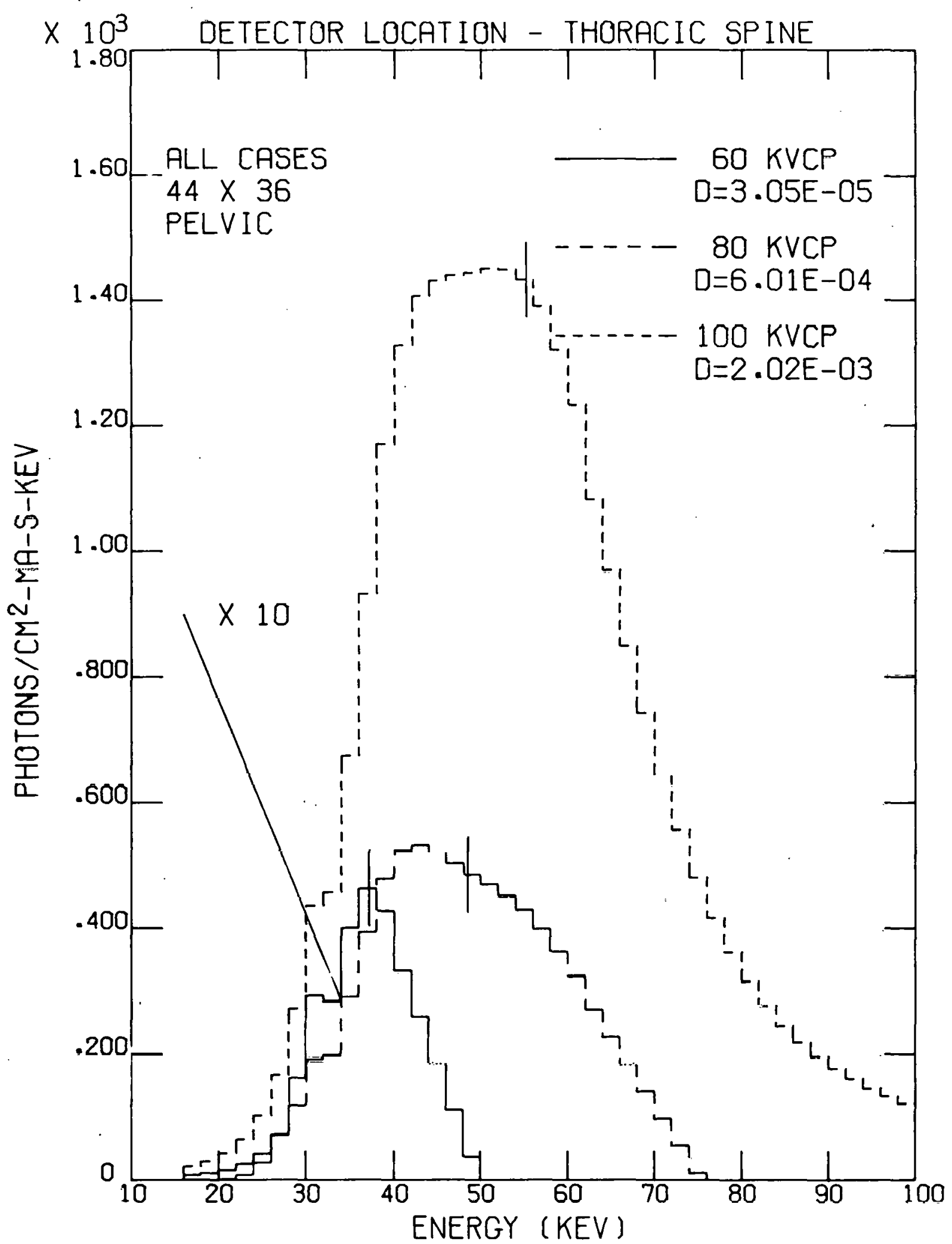

Pigure 94. Spectrum at the Thoracic Spine for Pelvic Exam. Vertical ticks indicate mean energy. Field size is in $\mathrm{cm}$. D is the absorbed dose per unit current per unit time (mrad/mA-s). 


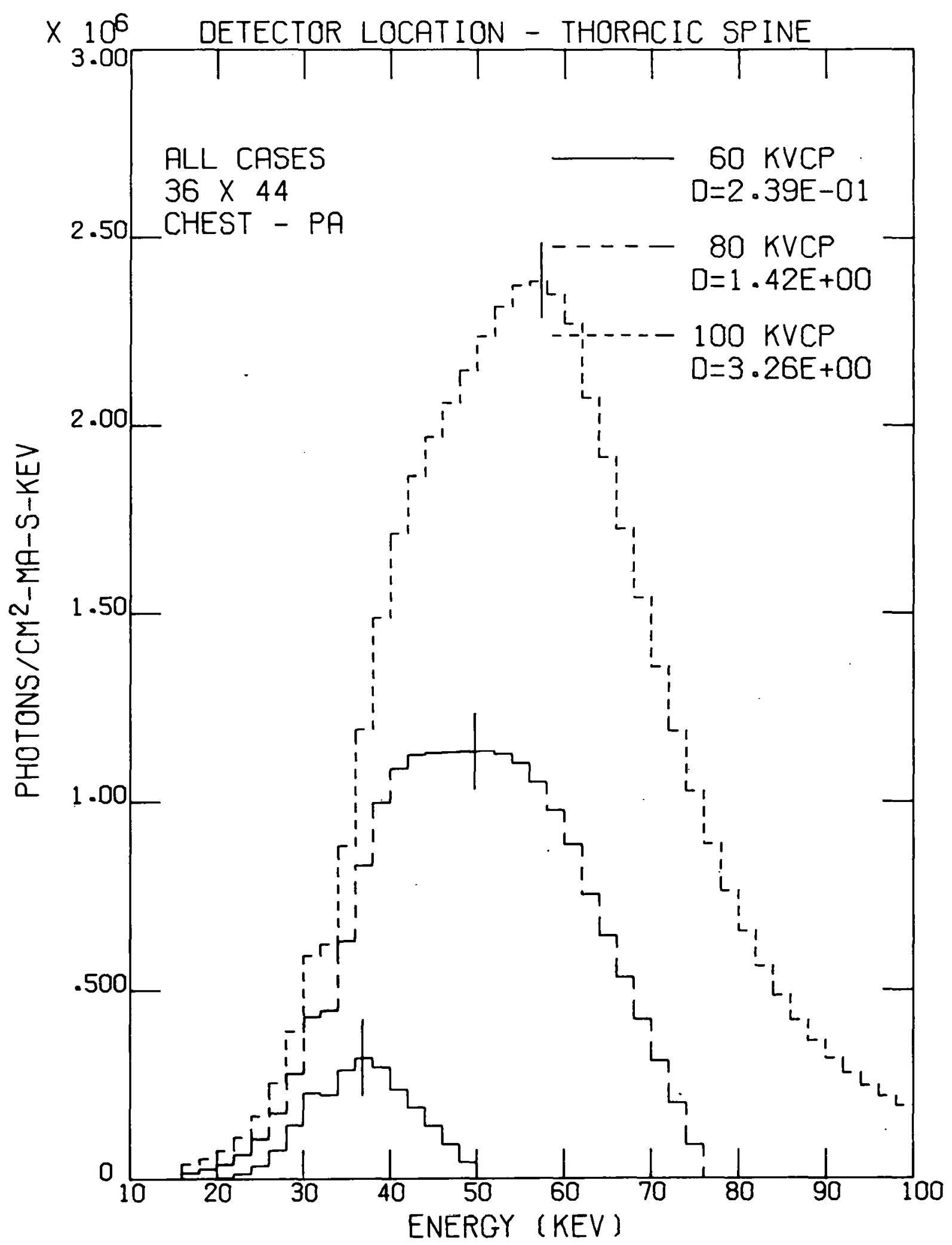

Figure 95. Spectrum at the Thoracic Spine for Chest Exam. Vertical ticks indicate mean energy. Field size is in $\mathrm{cm}$. D is the absorbed dose per unit current per unit time (mrad/mA-s). 


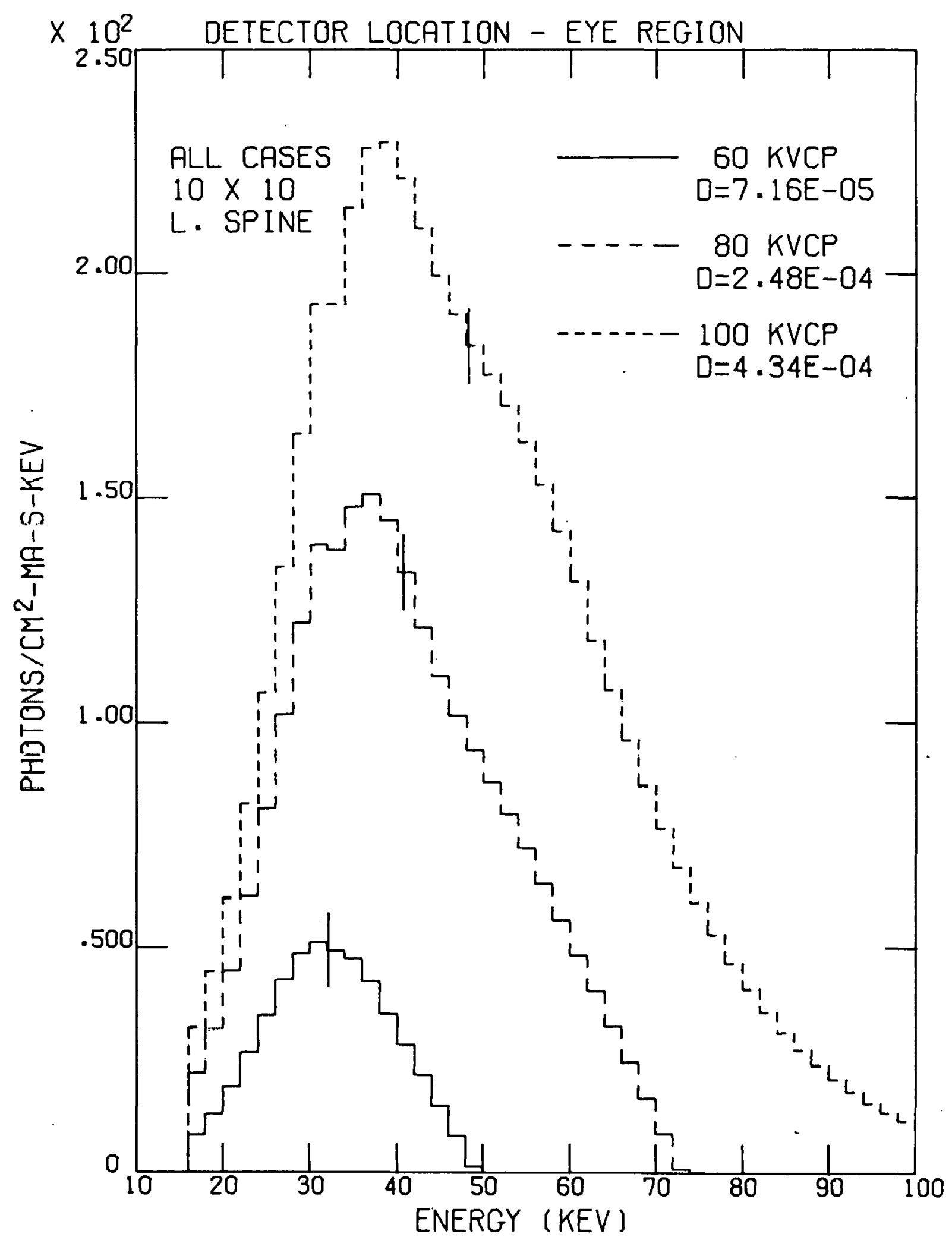

Figure 96. Spectrum at the Eye Region for Lower Spine Exam. Vertical ticks indicate mean energy. Field size is in $\mathrm{cm}$. D is the absorbed dose per unit current per unit time (mrad/mA-s). 


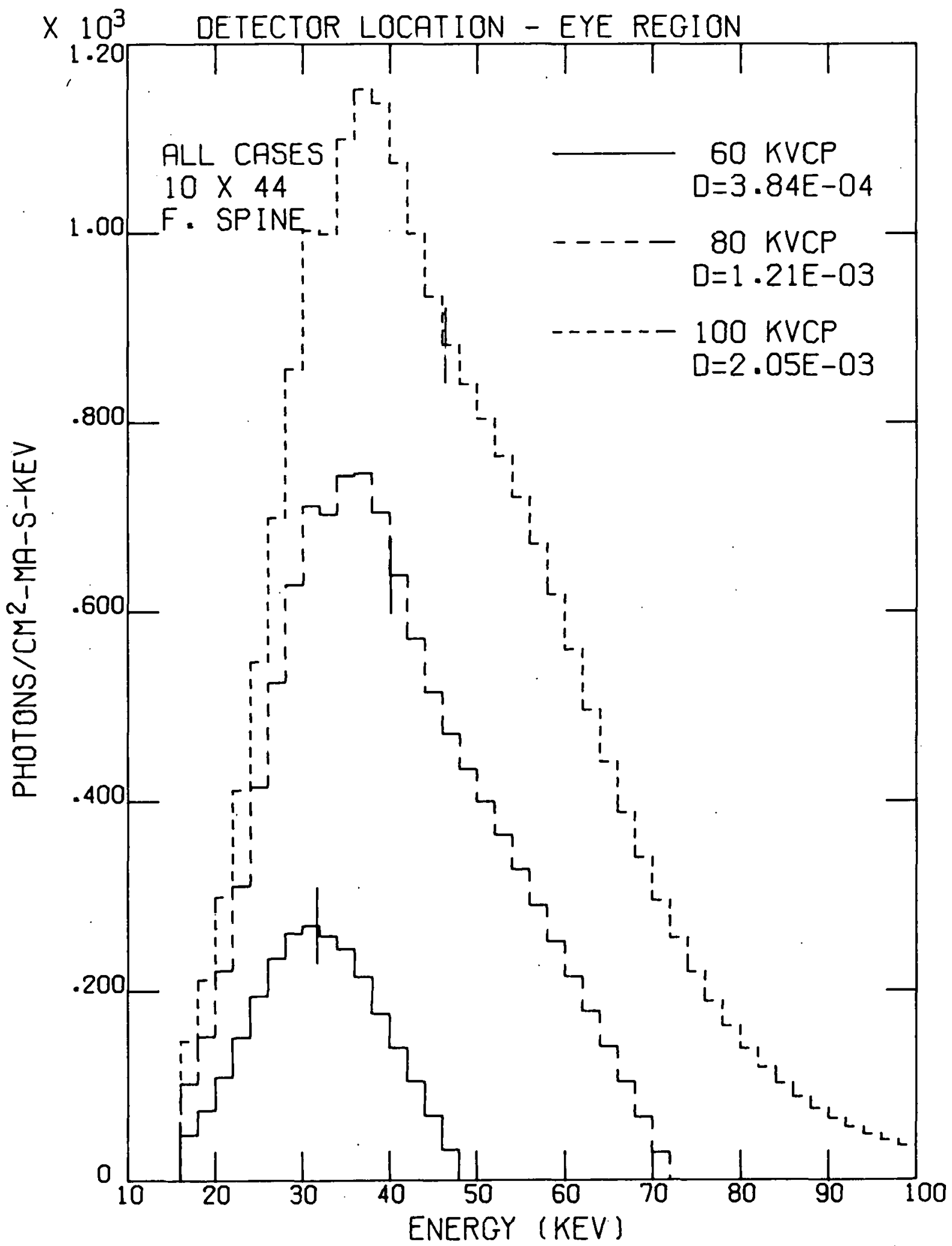

Figure 97. Spectrum at the Eye Region for Full Spine Exam. Vertical ticks indicate mean energy. Field size is in $\mathrm{cm}$. $D$ is the absorbed dose per unit current per unit time (mrad/mA-s). 


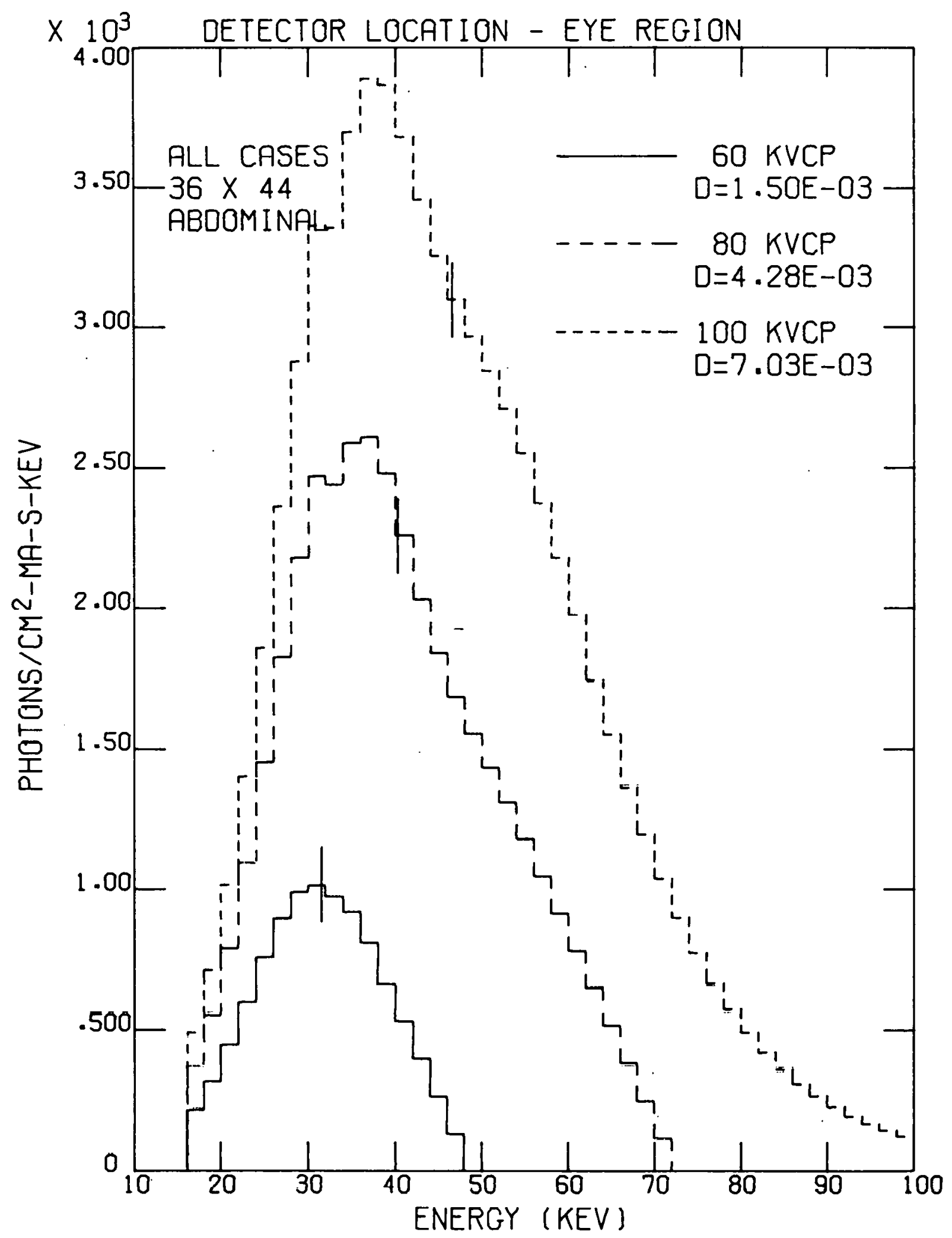

Figure 98. Spectrum at the Eye Region for Abdominal Exam. Vertical ticks indicate mean energy. Field size is in $\mathrm{cm}$. $D$ is the absorbed dose per unit current per unit time (mrad/mA-s). 


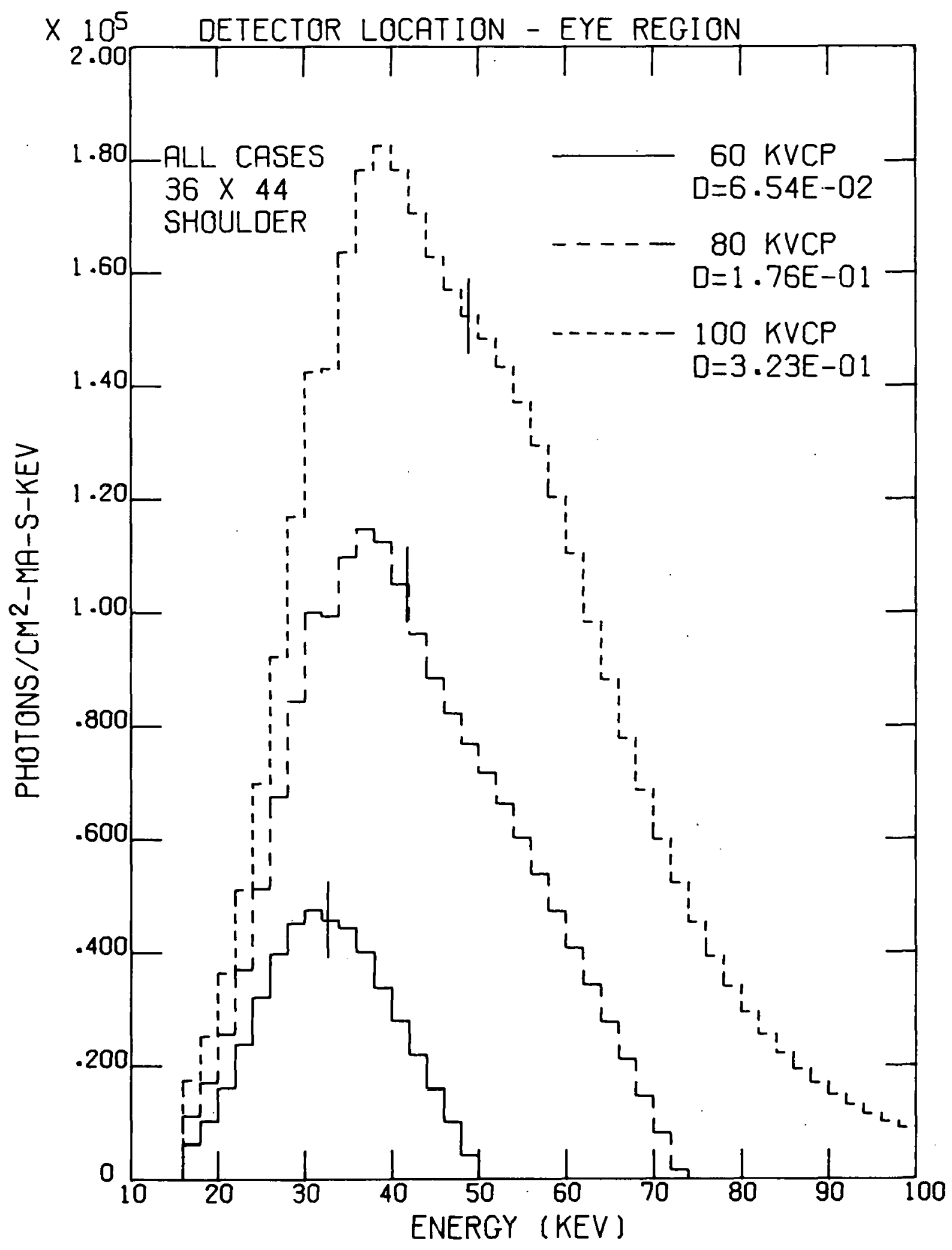

Figure 99. Spectrum at the Eye Region for Shoulder Exam. Vertical ticks indicate mean energy. Field size is in $\mathrm{cm}$. D is the absorbed dose per unit current per unit time (mrad/mA-s). 


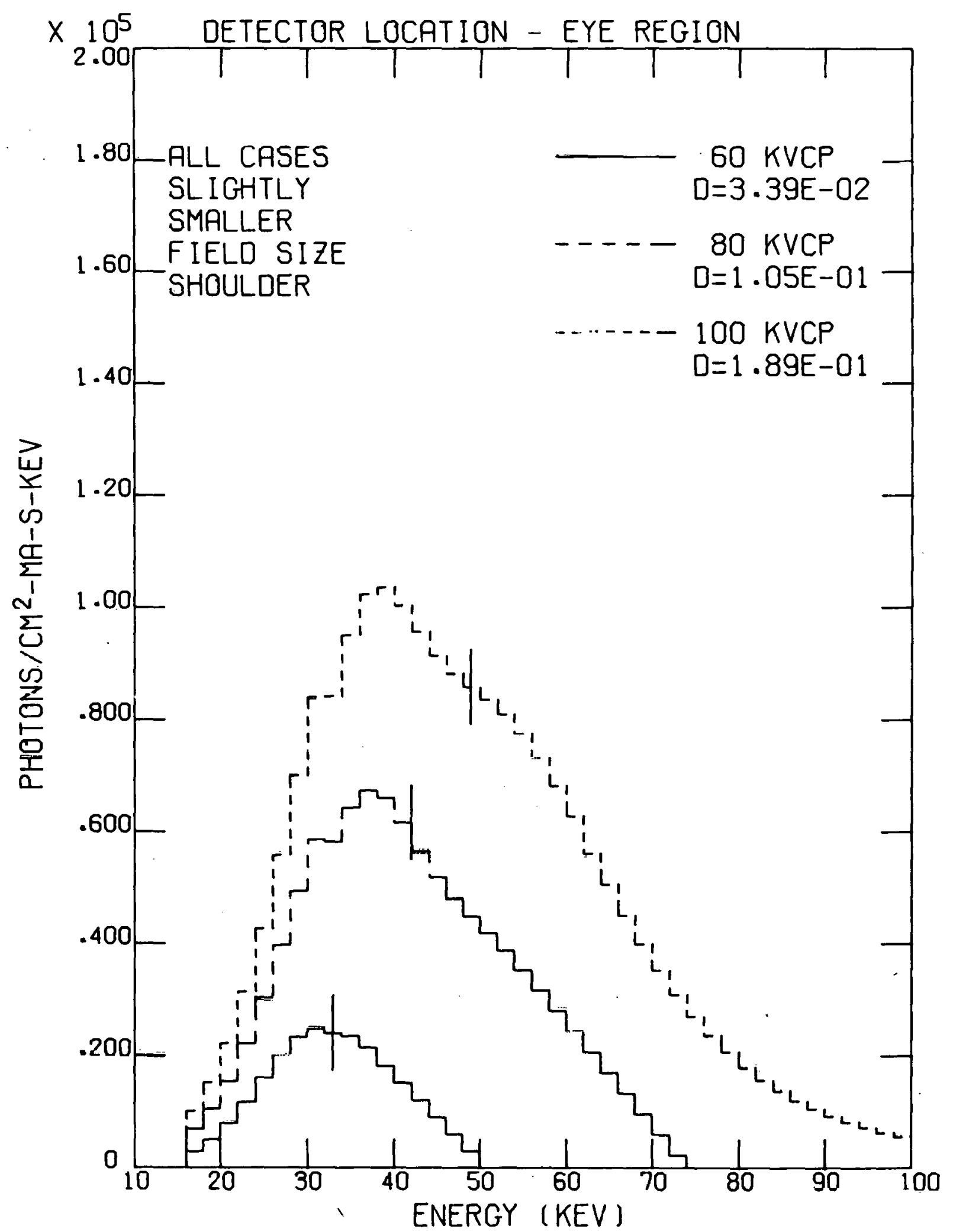

Figure 100. Spectrum at the Eye Region for Shoulder Exam (with $28 \times 44$ $\mathrm{cm}$ Field). Vertical ticks indicate mean energy. Field size is in $\mathrm{cm}$. $D$ is the absorbed dose per unit current per unit time $(\mathrm{mrad} / \mathrm{mA}-\mathrm{s})$. 


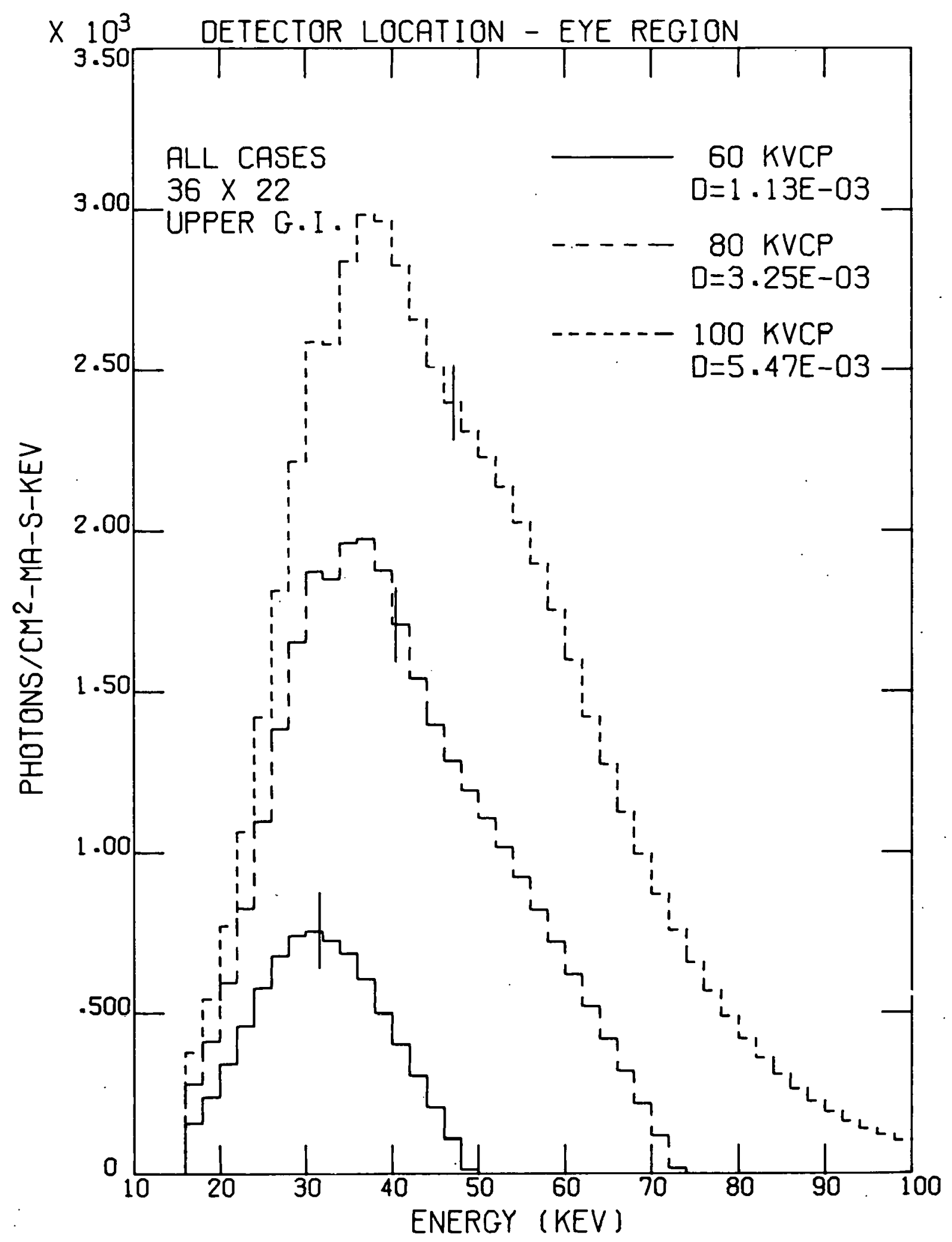

Figure 101. Spectrum at the Eye Region for Upper G.I. Exam. Vertical ticks indicate mean energy. Field size is in $\mathrm{cm}$. D is the absorbed dose per unit current per unit time (mrad/mA-s). 


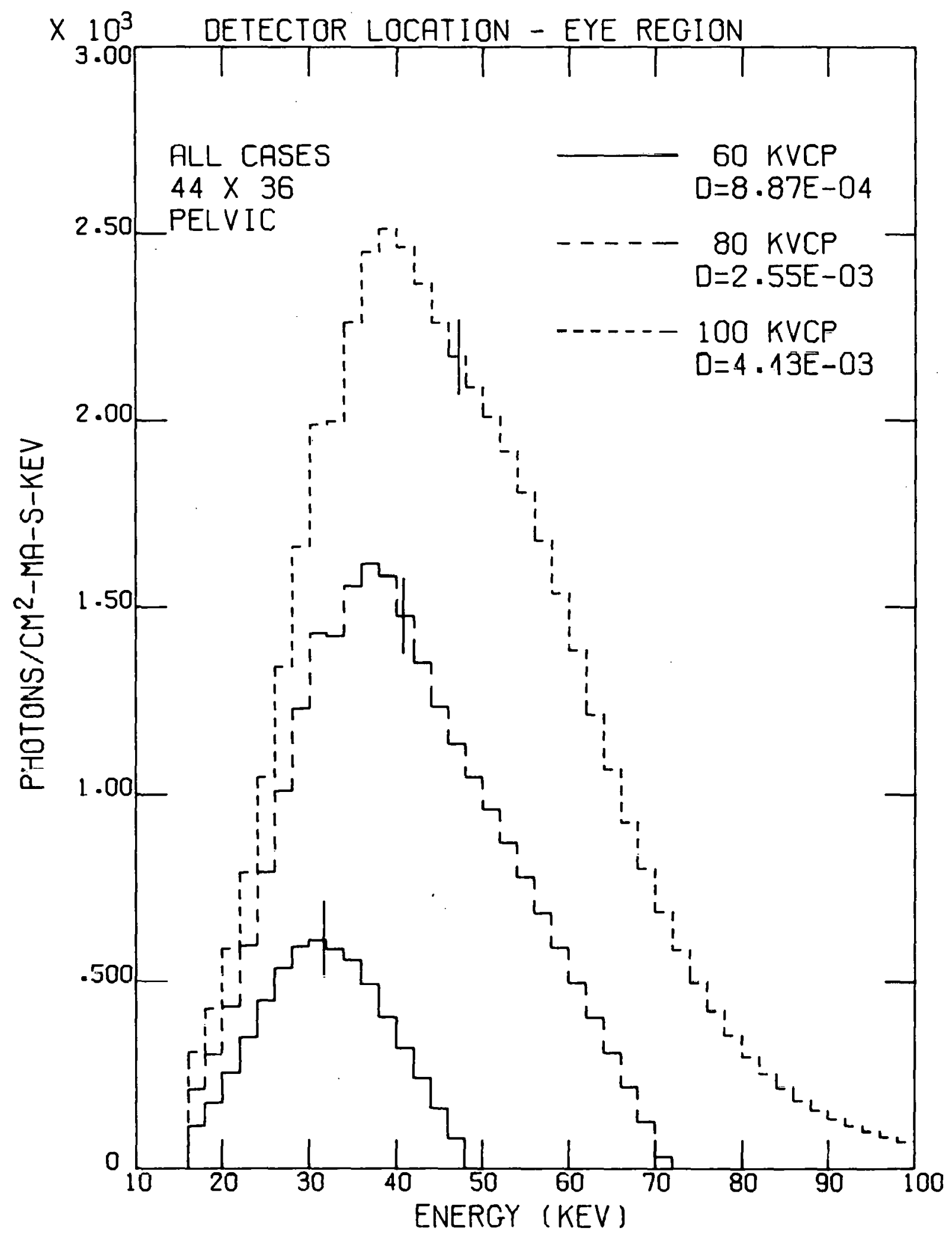

Figure 102. Spectrum at the Eye Region for Pelvic Exam. Vertical ticks indicate mean energy. Field size is in $\mathrm{cm}$. D is the absorbed dose per unit current per unit time (mrad/mA-s). 


\section{APPENDIX B \\ EFFECT OF FIELD SIZE}

Presented in this Appendix are the comparisons of the spectra acquired for the lower spine, full spine, and abdominal examinations for six detector locations including the testes, right ovary, thyroid, lens of the eye, fifth lumbar vertebra, and thoracic spine regions. These comparisons are presented to show the effect of field size on the spectrum. The lower spine, full spine, and abdominal exams all have the same beam center, and their field sizes are $10 \times 10 \mathrm{~cm}$, $10 \times 44 \mathrm{~cm}$, and $36 \times 44 \mathrm{~cm}$, respectively (see Figures 22-24). 


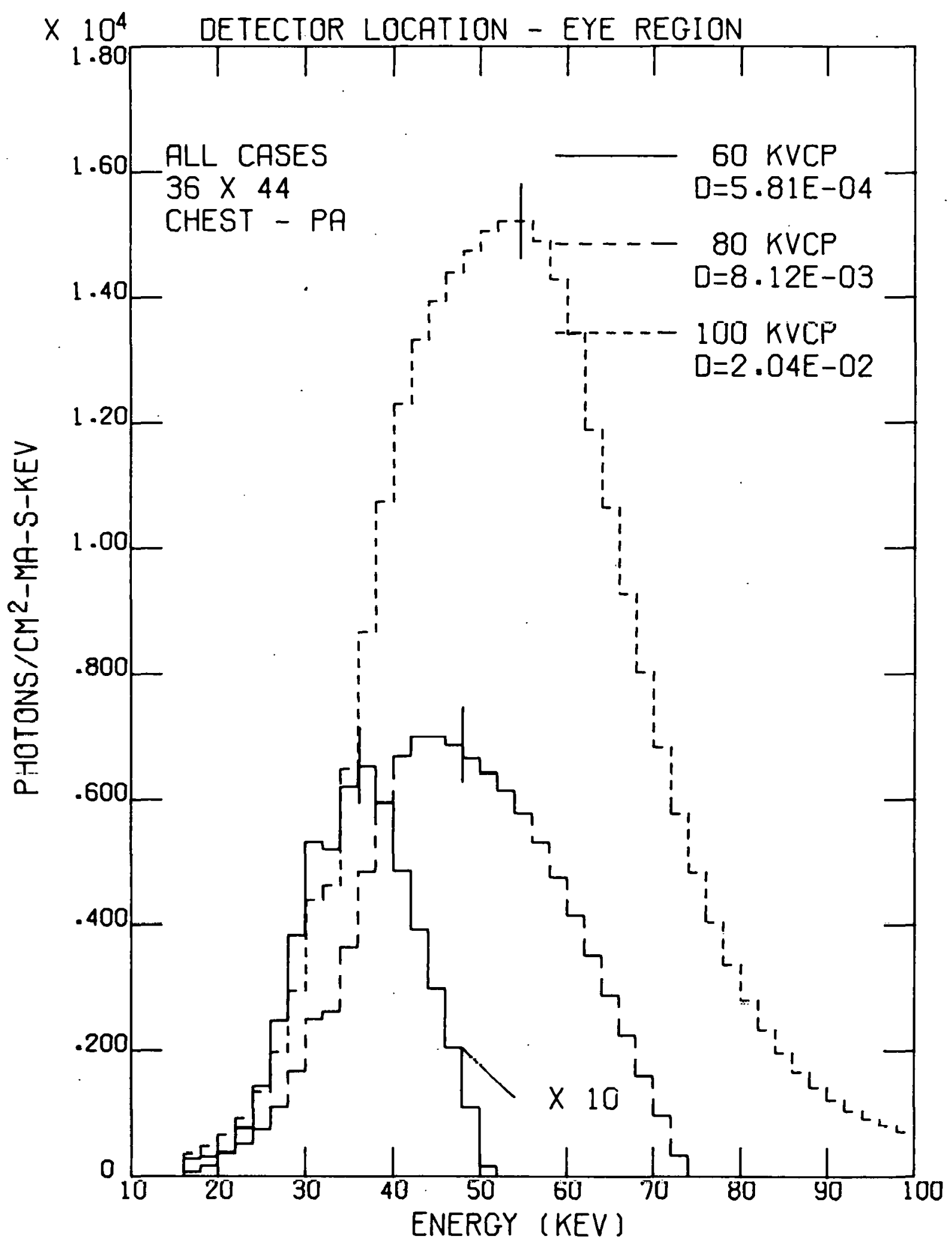

Figure 103. Spectrum at the Eye Region for Chest Exam. Vertical ticks indicate mean energy. Field size is in $\mathrm{cm}$. $D$ is the absorbed dose per unit current per unit time ( $\mathrm{mrad} / \mathrm{mA}-\mathrm{s})$. 


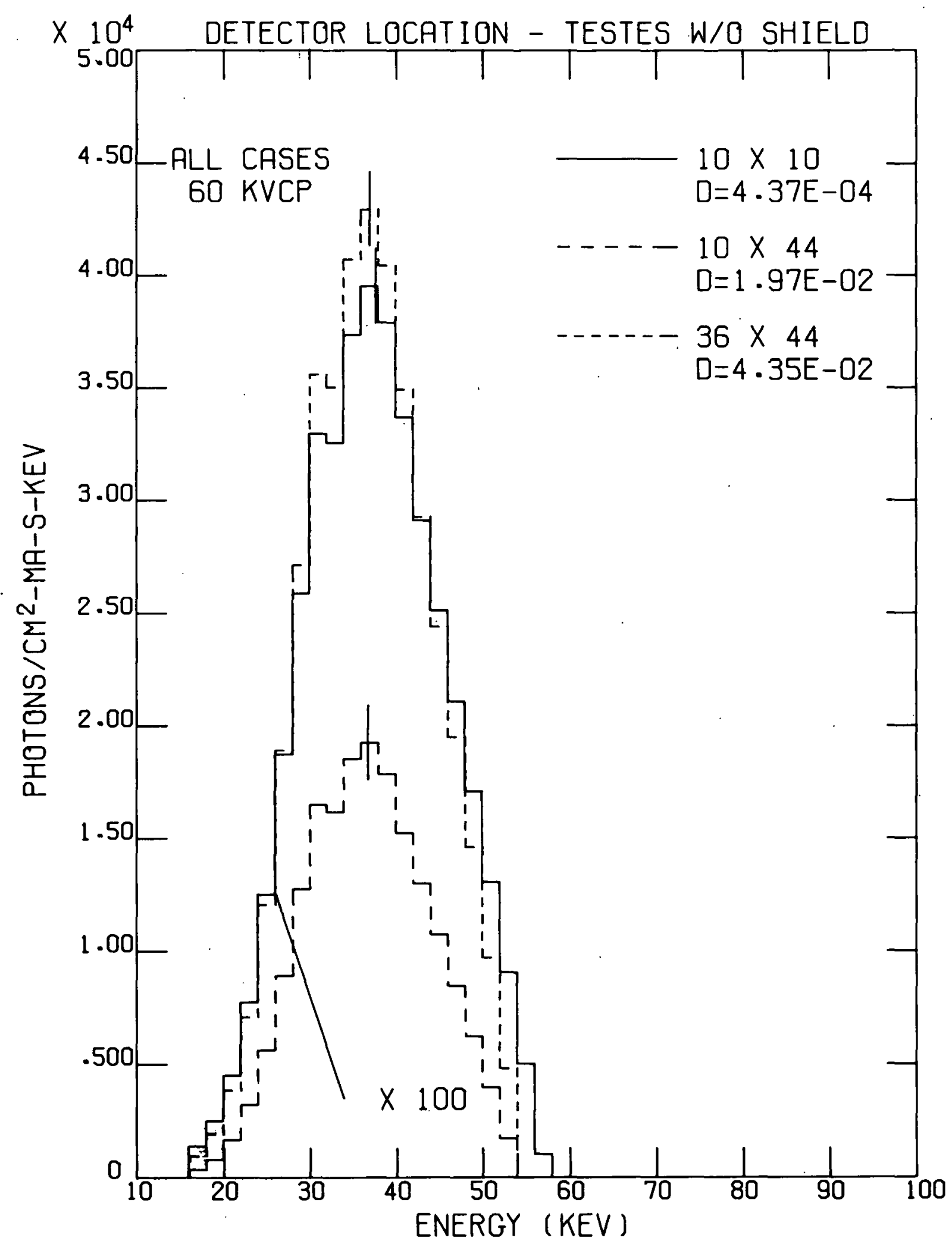

Figure 104. Spectrum at the Testes as a Function of Field Size -- $60 \mathrm{kVcp}$. Vertical ticks indicate mean energy. Field size is in $\mathrm{cm}$. $D$ is the absorbed dose per unit current per unit time (mrad/mA-s). 


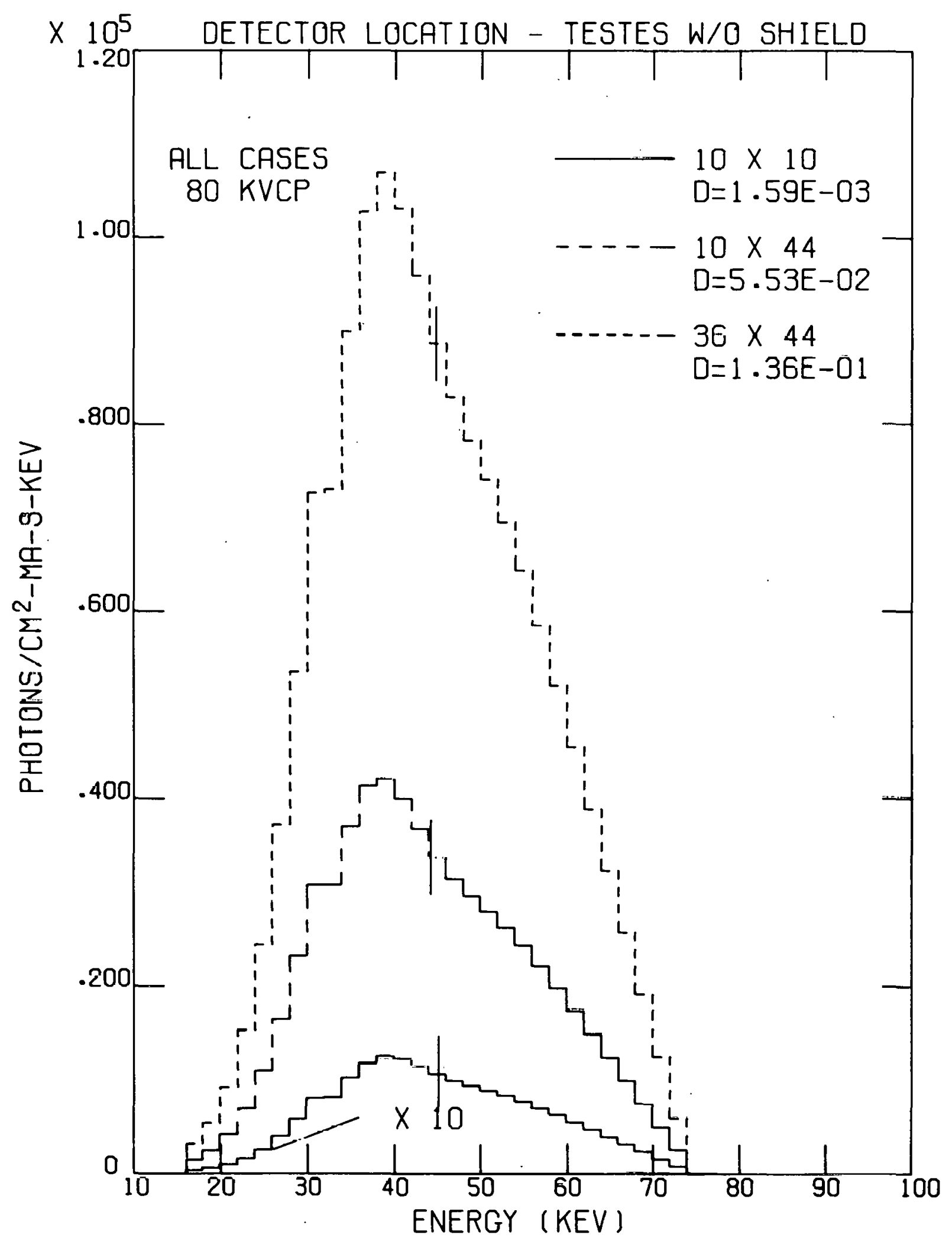

Figure 105. Spectrum at the Testes as a Function of Field Size $--80 \mathrm{kVcp}$. Vertical ticks indicate mean energy. Field size is in $\mathrm{cm}$. $D$ is the absorbed dose per unit current per unit time $(\mathrm{mrad} / \mathrm{mA}-\mathrm{s})$. 


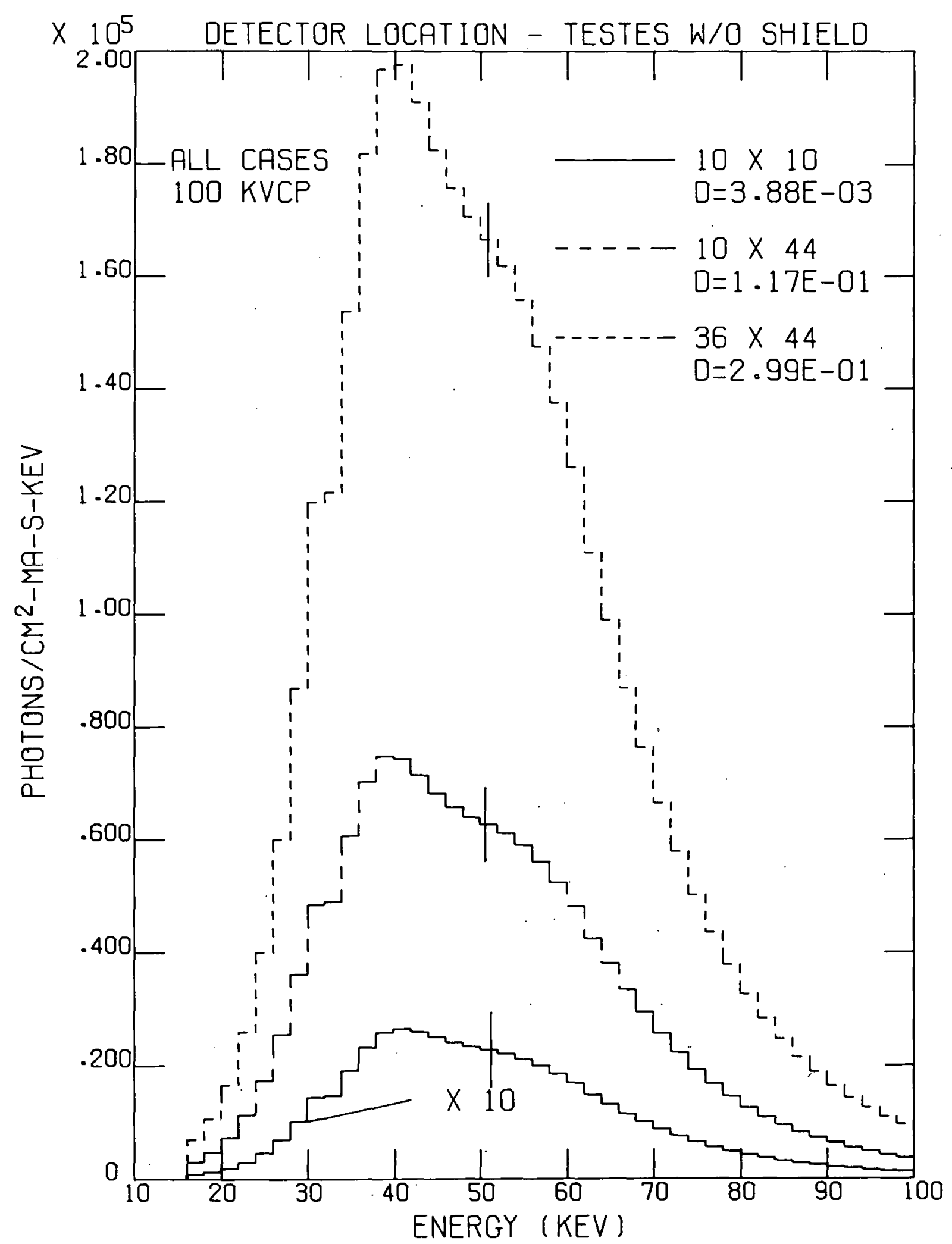

Figure 106. Spectrum at the Testes as a Function of Field Size -$100 \mathrm{kV} c \mathrm{p}$. Vertical ticks indicate mean energy. Field size is in $\mathrm{cm}$. $D$ is the absorbed dose per unit current per unit time $(\mathrm{mrad} / \mathrm{mA}-\mathrm{s})$. 


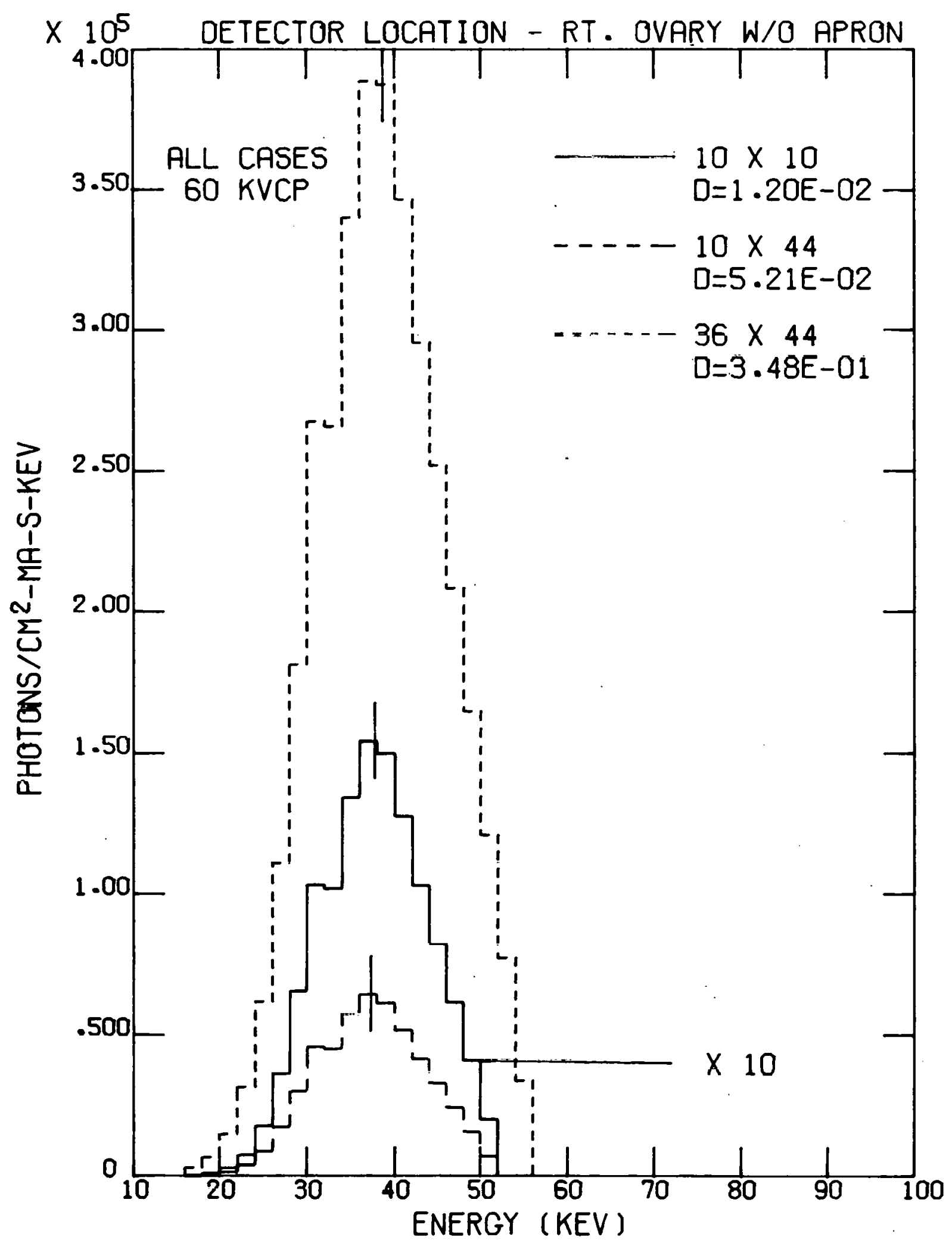

Figure 107. Spectrum at the Right Ovary as a Function of Field Size -$60 \mathrm{kVcp}$. Vertical ticks indicate mean energy. Field size is in $\mathrm{cm}$. $D$ is the absorbed dose per unit current per unit time $(\mathrm{mrad} / \mathrm{mA}-\mathrm{s})$. 


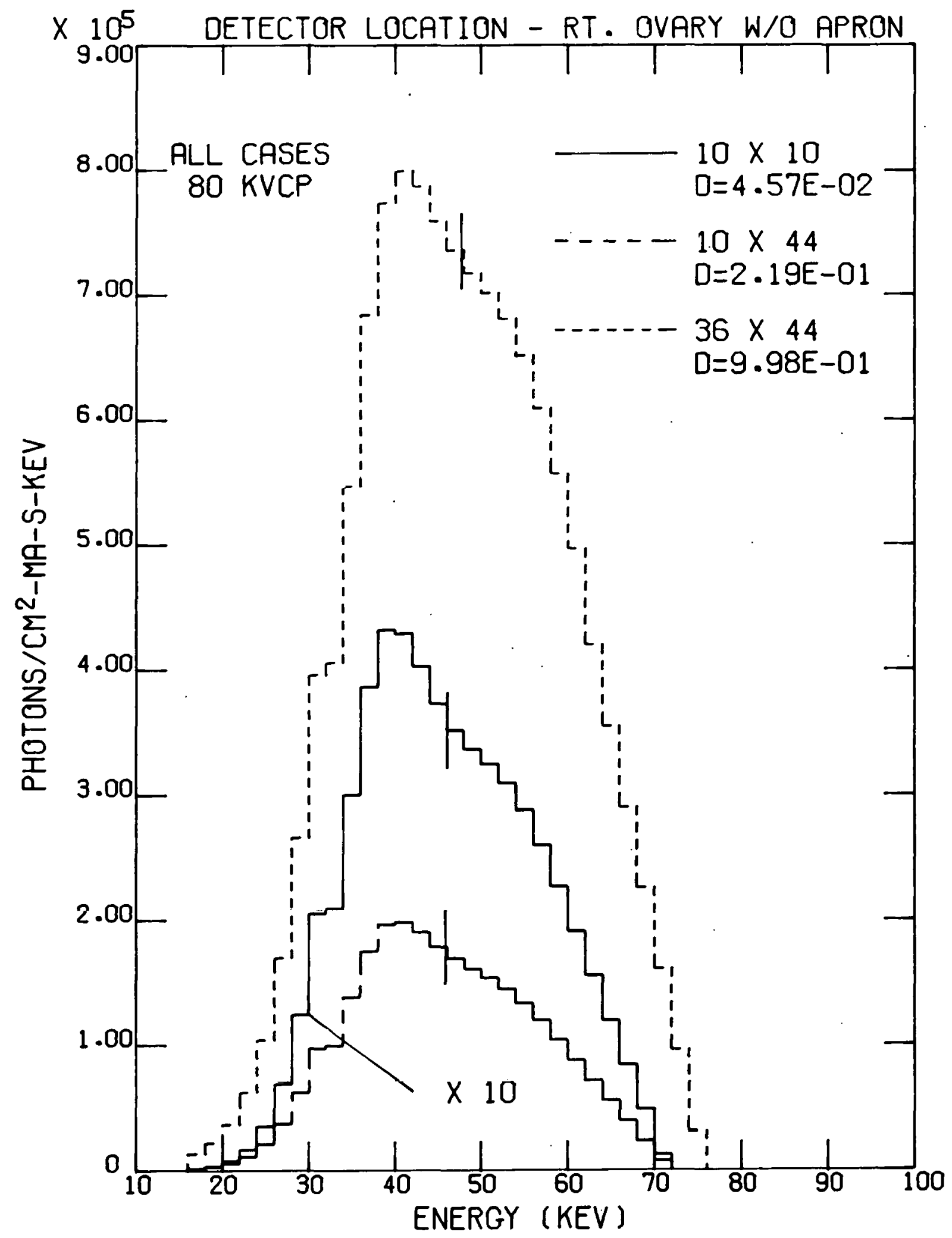

Figure 108. Spectrum at the Right Ovary as a Function of Field Size -$80 \mathrm{kVcp}$. Vertical ticks indicate mean energy. Field size is in $\mathrm{cm}$. $D$ is the absorbed dose per unit current per unit time $(\mathrm{mrad} / \mathrm{mA}-\mathrm{s})$. 


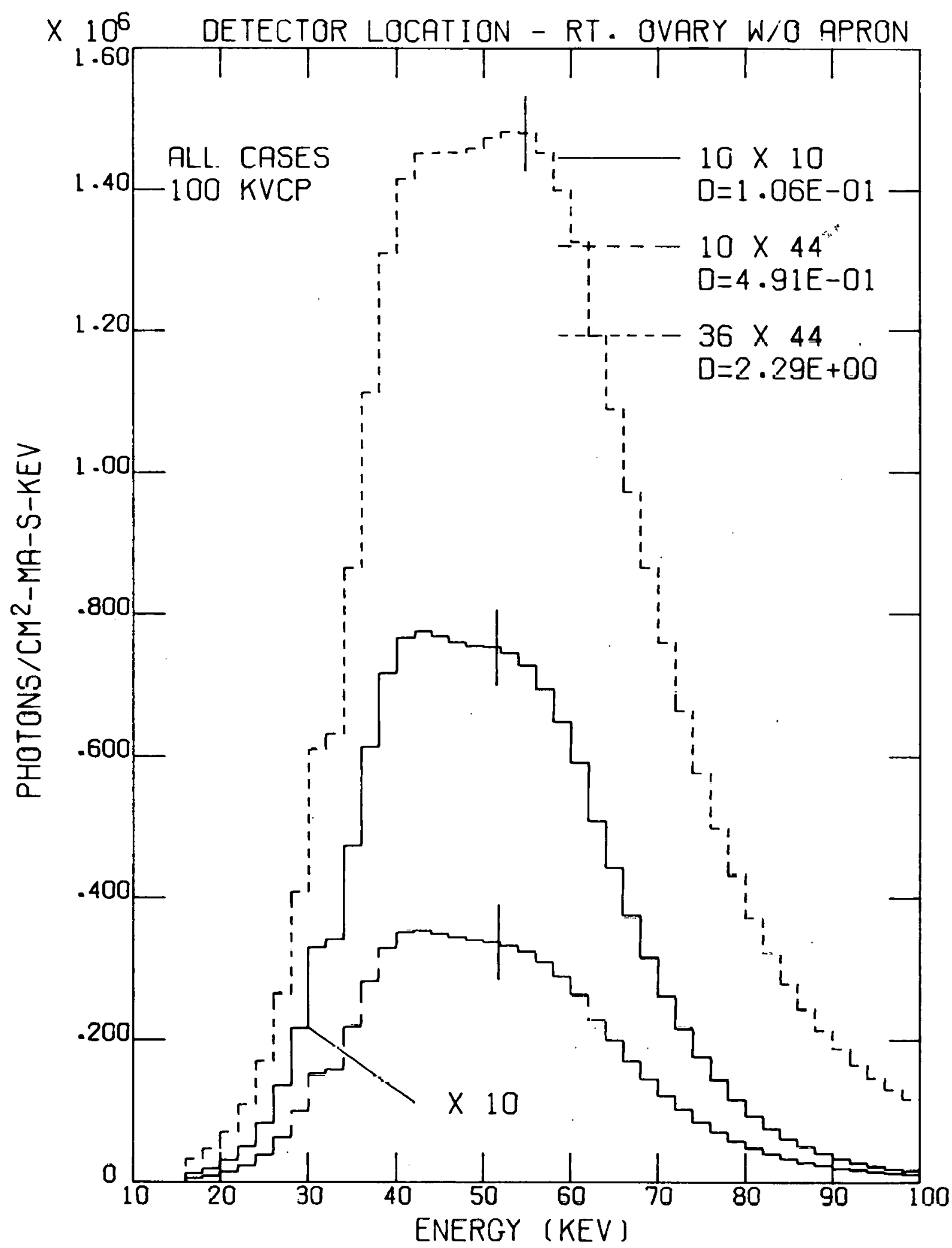

Figure 109. Spectrum at the Right Ovary as a Function of Field Size -$100 \mathrm{kVcp}$. Vertical ticks indicate mean energy. Field size is in $\mathrm{cm}$. D is the absorbed dose per unit current per unit time $(\mathrm{mrad} / \mathrm{mA}-\mathrm{s})$. 


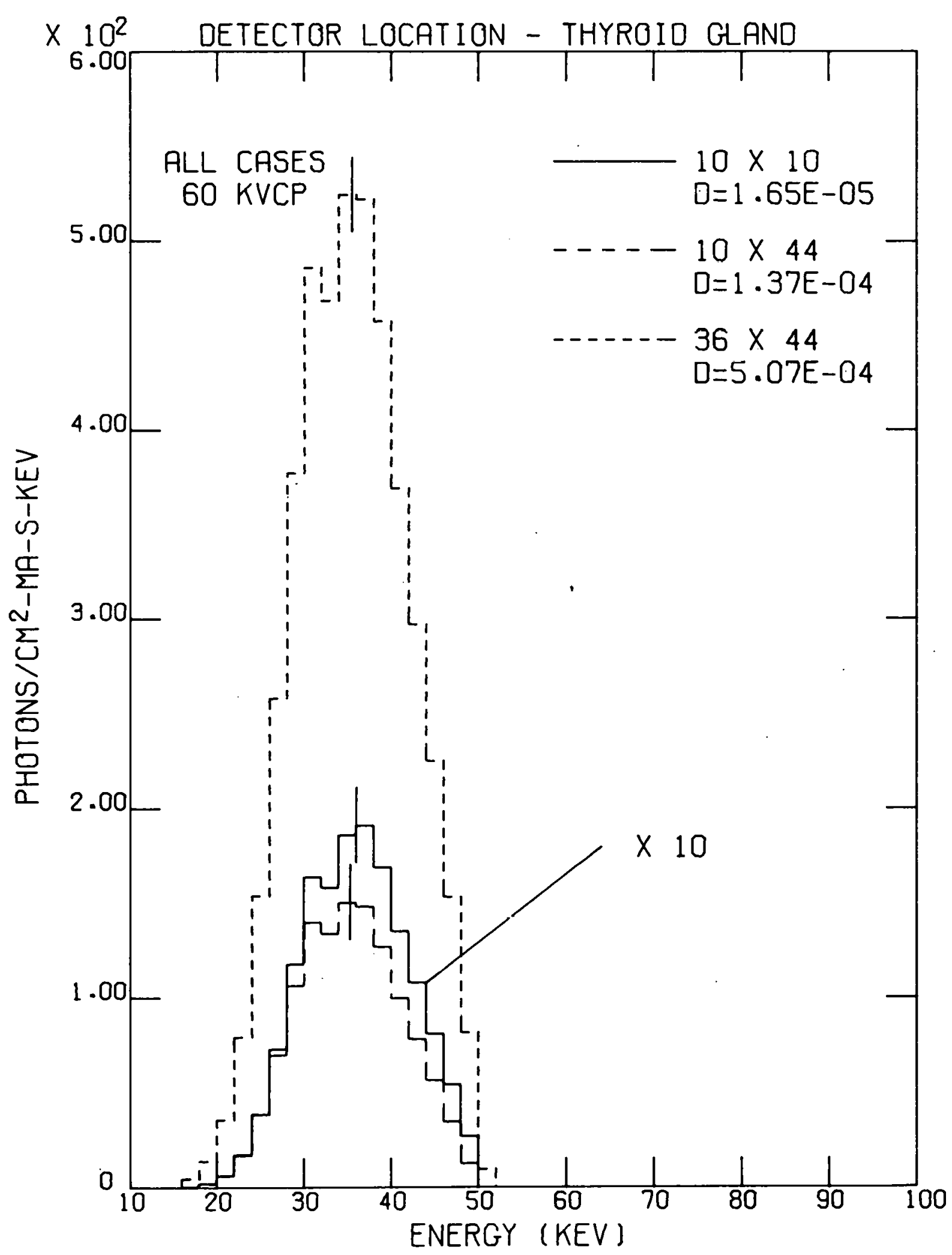

Figure 110. Spectrum at the Thyroid as a Function of Field Size -$60 \mathrm{kVcp}$. Vertical ticks indicate mean energy. Field size is in $\mathrm{cm}$. $D$ is the absorbed dose per unit current per unit time $(\mathrm{mrad} / \mathrm{mA}-\mathrm{s})$. 


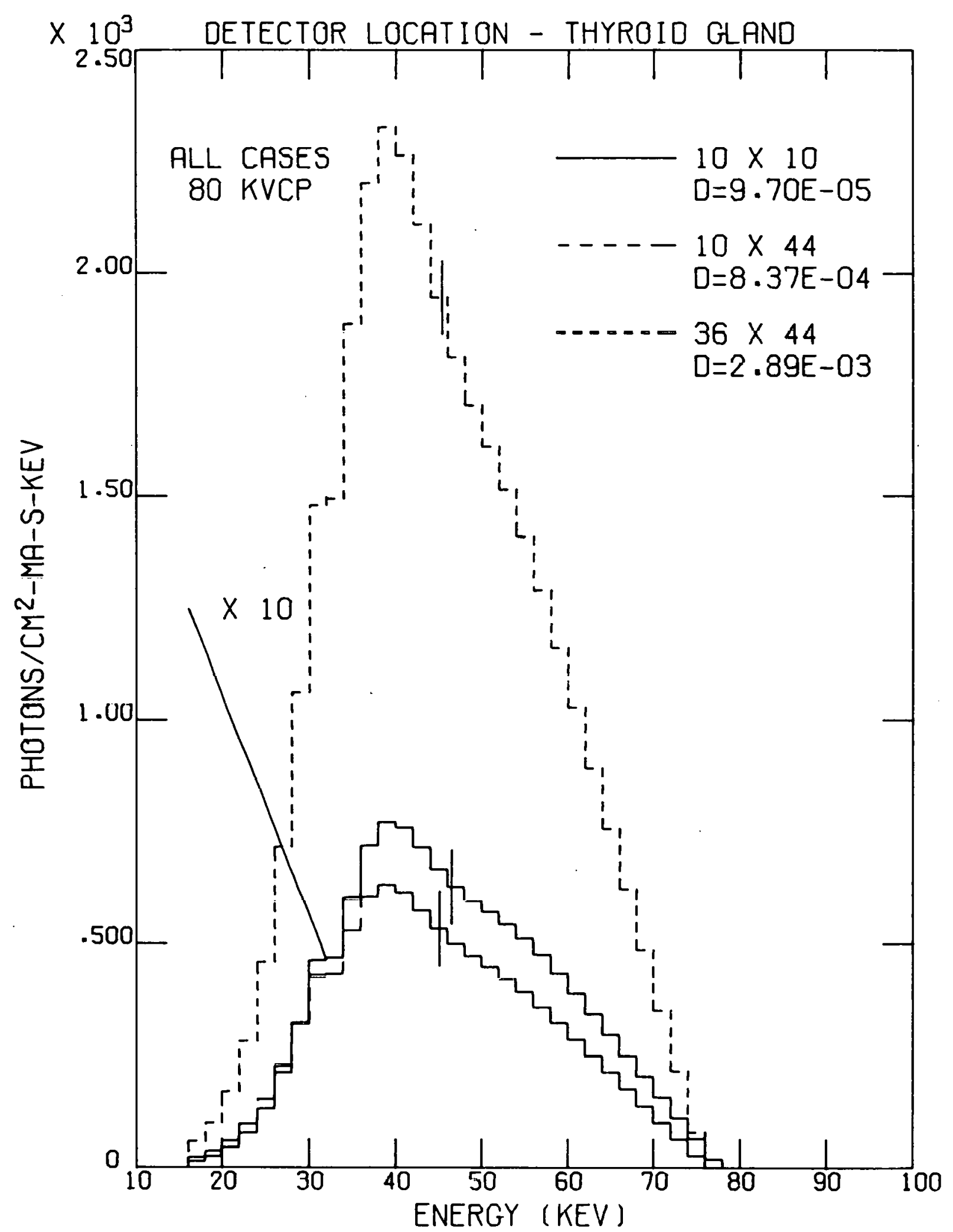

Figure 111. Spectrum at the Thyroid as a Function of Field Size -$80 \mathrm{kVcp}$. Vertical ticks indicate mean energy. Field size is in $\mathrm{cm}$. $D$ is the absorbed dose per unit current per unit time $(\mathrm{mrad} / \mathrm{mA}-\mathrm{s})$. 


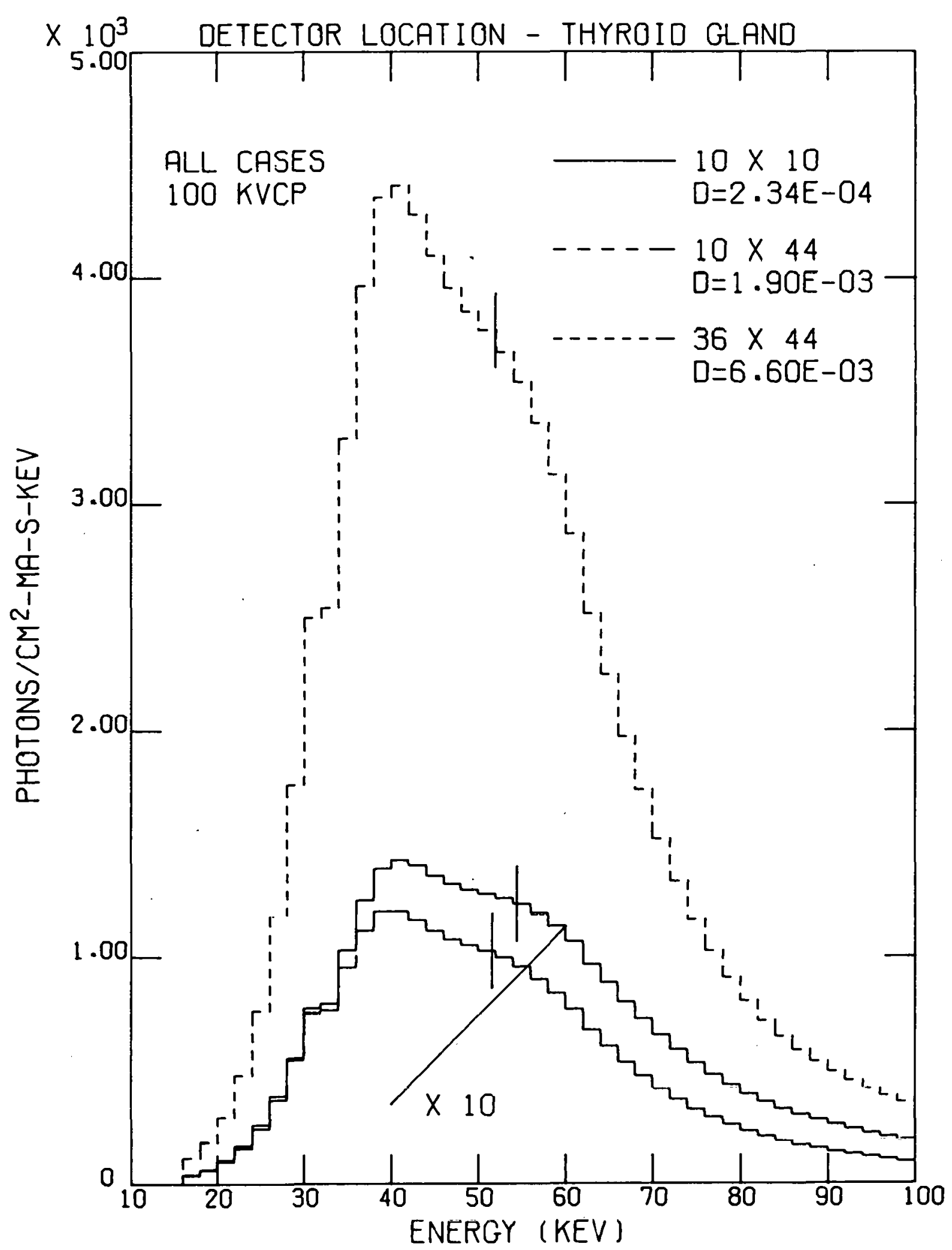

Figure 112. Spectrum at the Thyroid as a Function of Field Size -$100 \mathrm{kVcp}$. Vertical ticks indicate mean energy. Field size is in $\mathrm{cm}$. $D$ is the absorbed dose per unit current per unit time $(\mathrm{mrad} / \mathrm{mA}-\mathrm{s})$. 


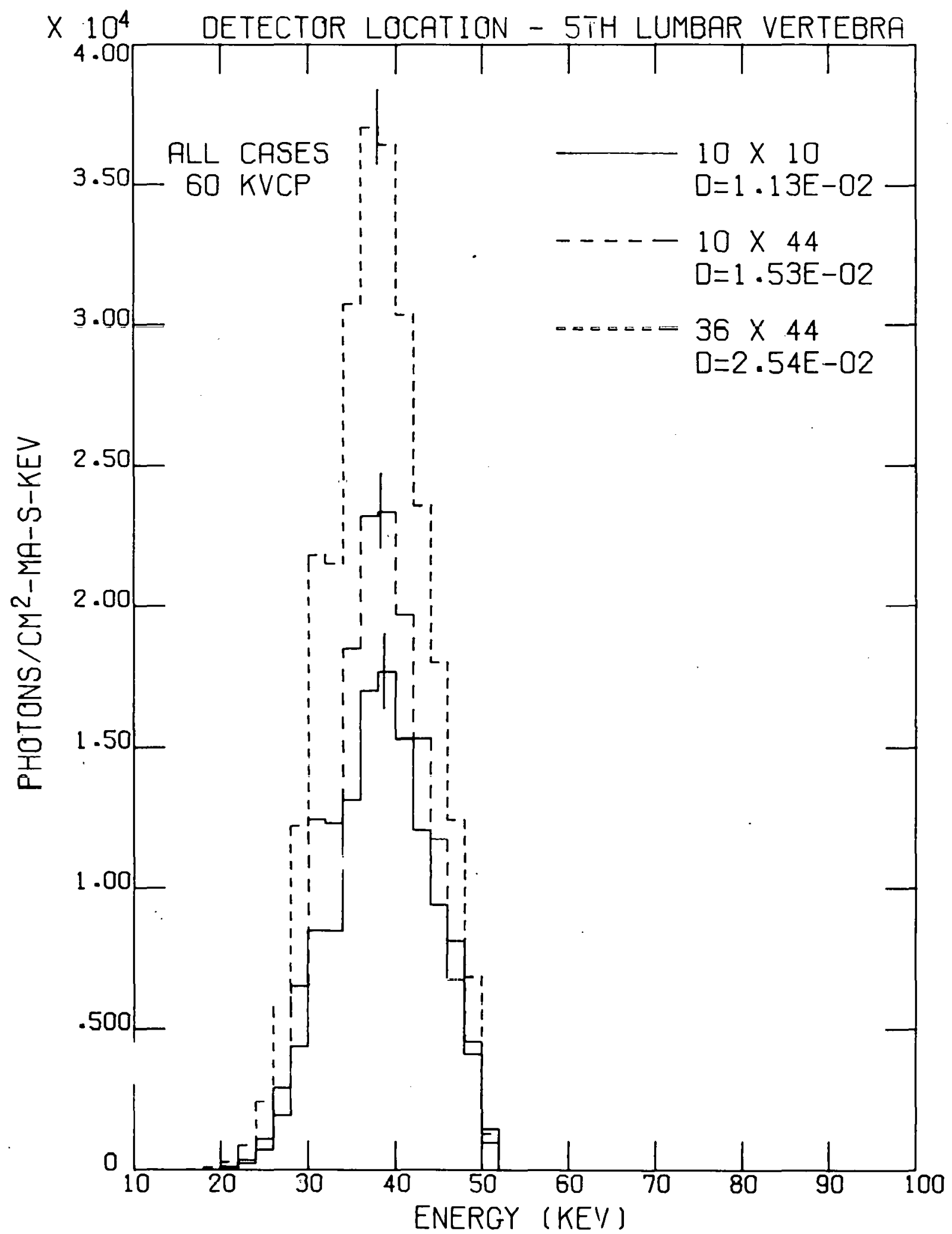

Figure 113. Spectrum at the Fifth Lumbar Vertebra as a Function of Field Size -- $60 \mathrm{kVcp}$. Vertical ticks indicate mean energy. Field size is in $\mathrm{cm}$. $D$ is the absorbed dose per unit current per unit time (mrad/mA-s). 


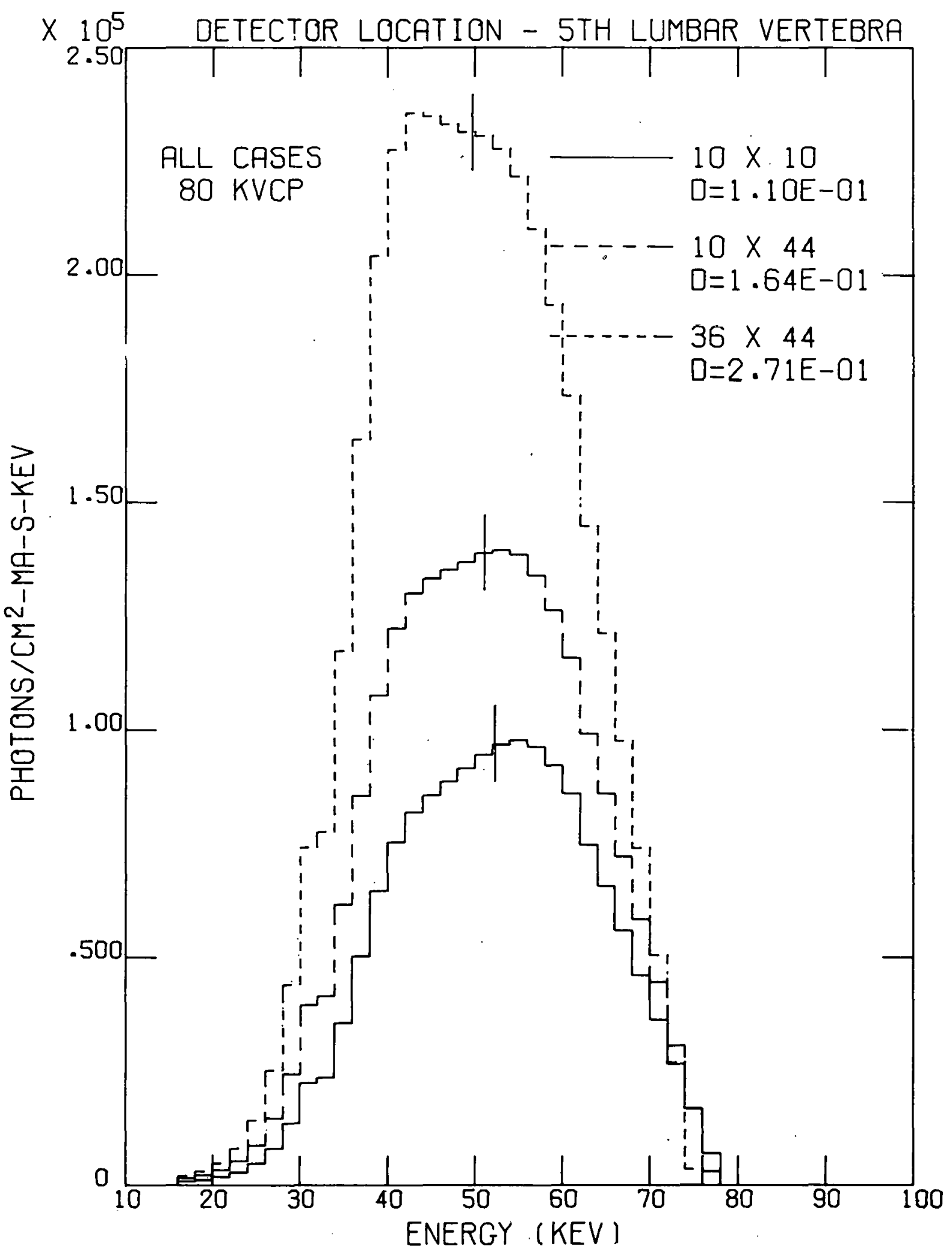

Figure 114. Spectrum at the Fifth Lumbar Vertebra as a Function of Field Size -- $80 \mathrm{kVcp}$. Vertical ticks indicate mean energy. Field size is in $\mathrm{cm}$. $\mathrm{U}$ is the absorbed dose per unit current per unit time (mrad/mA-s). 


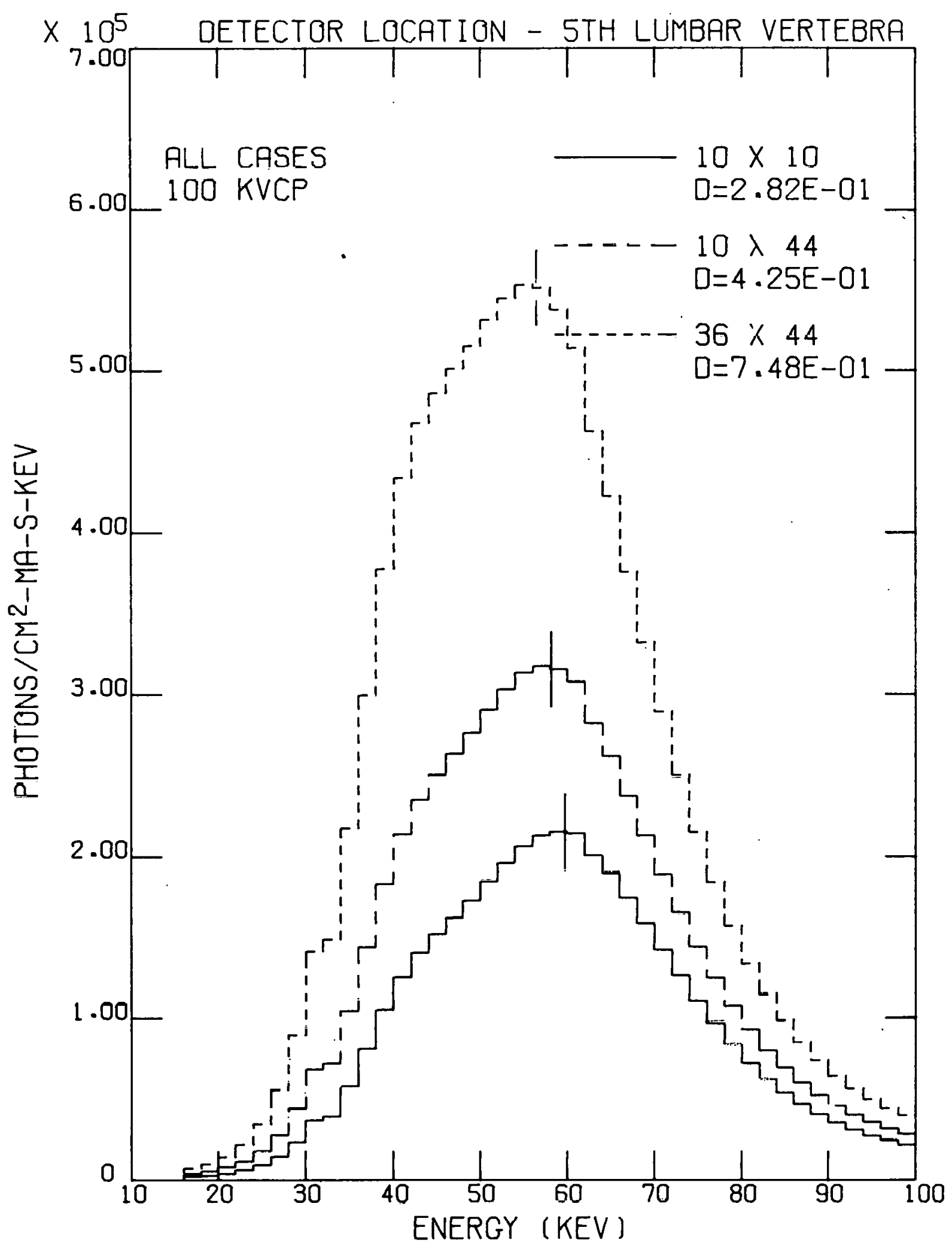

Figure 115. Spectrum at the Fifth Lumbar Vertebra as a Function of Field Size -- $100 \mathrm{kV} c p$. Vertical ticks indicate mean energy. Field size is in $\mathrm{cm}$. $D$ is the absorbed dose per unit current per unit time $(\mathrm{mrad} / \mathrm{mA}-\mathrm{s})$. 


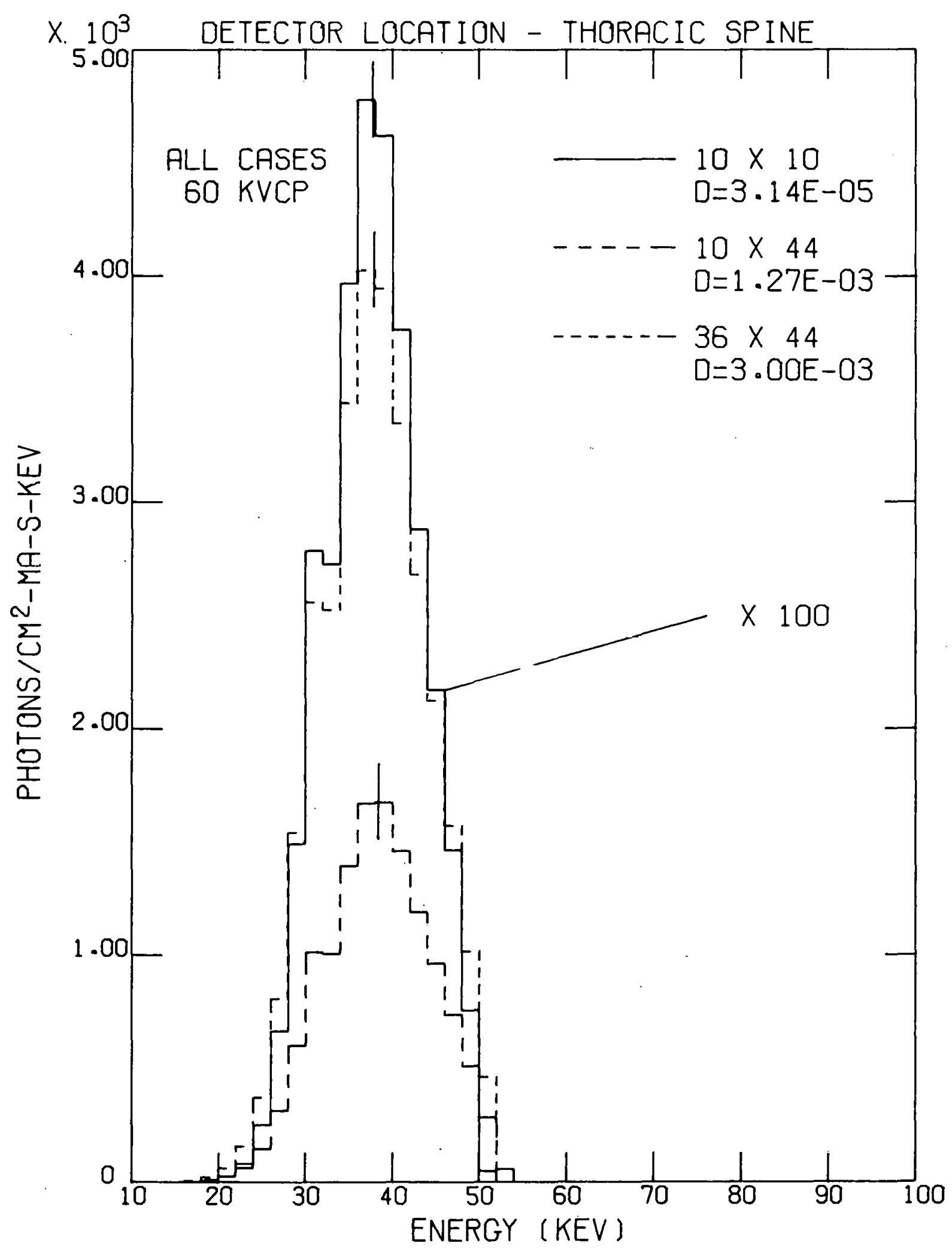

Figure 116. Spectrum at the Thoracic Spine as a Function of Field Size -- $60 \mathrm{kVcp}$. Vertical ticks indicate mean energy. Field size is in $\mathrm{cm}$. $D$ is the absorbed dose per unit current per unit time (mrad/mA-s). 


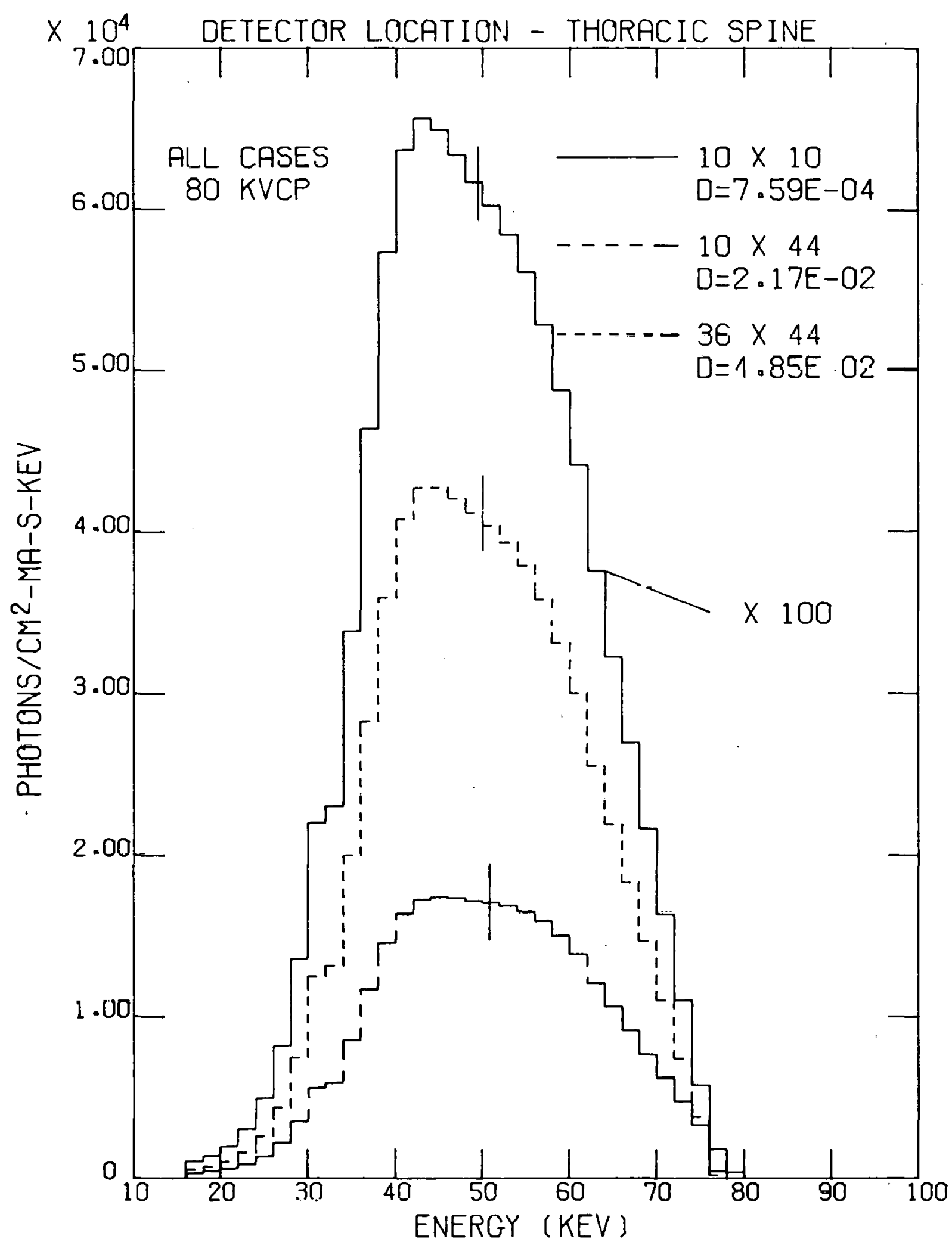

Figure 117. Spectrum at the Thoracic Spine as a Function of Field Size -- $80 \mathrm{kVcp}$. Vertical ticks indicate mean energy. Field size is in $\mathrm{cm}$. $D$ is the absorbed dose per unit current per unit time (mrad/mA-s). 


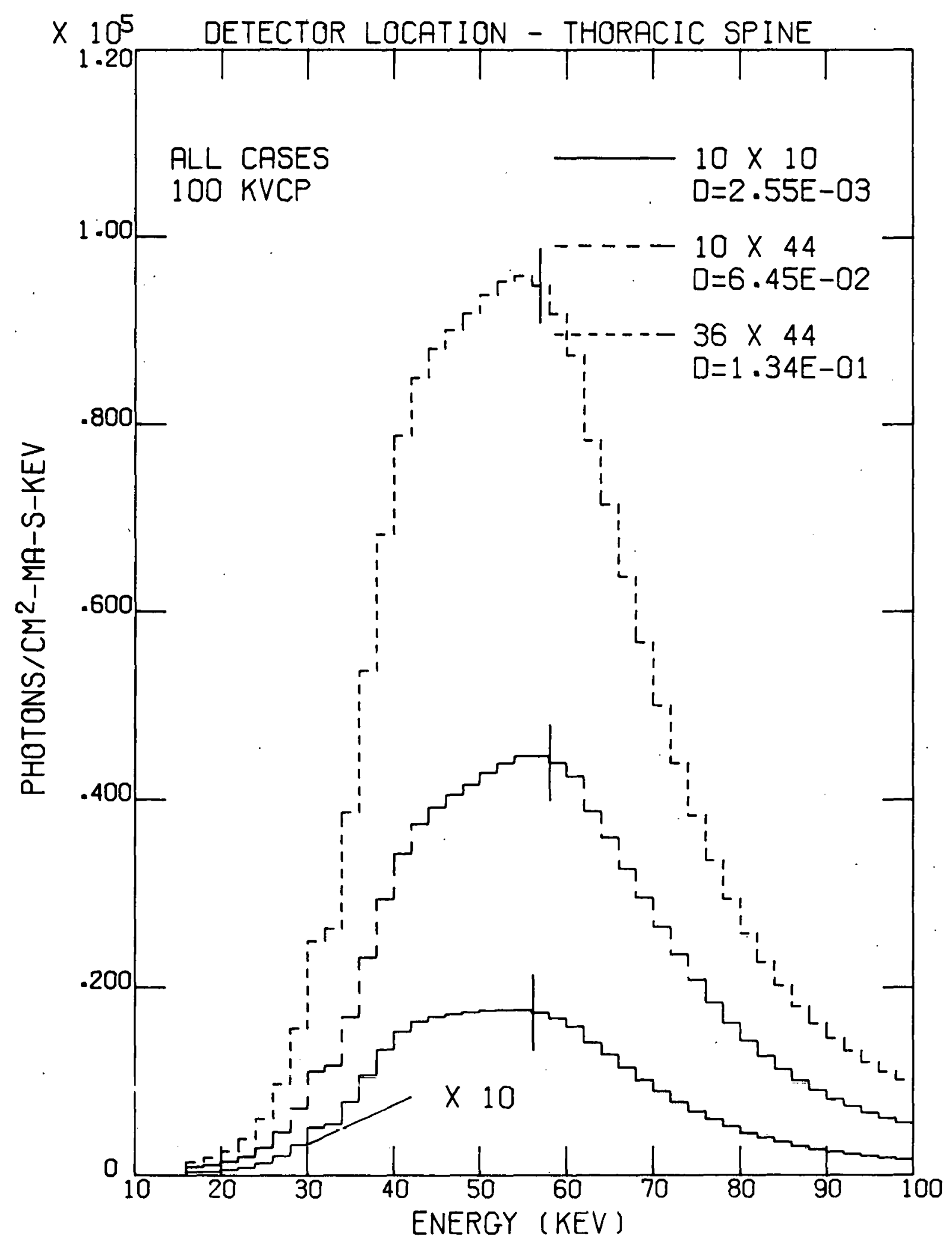

Figure 118. Spectrum at the Thoracic Spine as a Function of Field Size -- $100 \mathrm{kVcp}$. Vertical ticks indicate mean energy. Field size is in $\mathrm{cm}$. D is the absorbed dose per unit current per unit time (mrad/mA-s). 


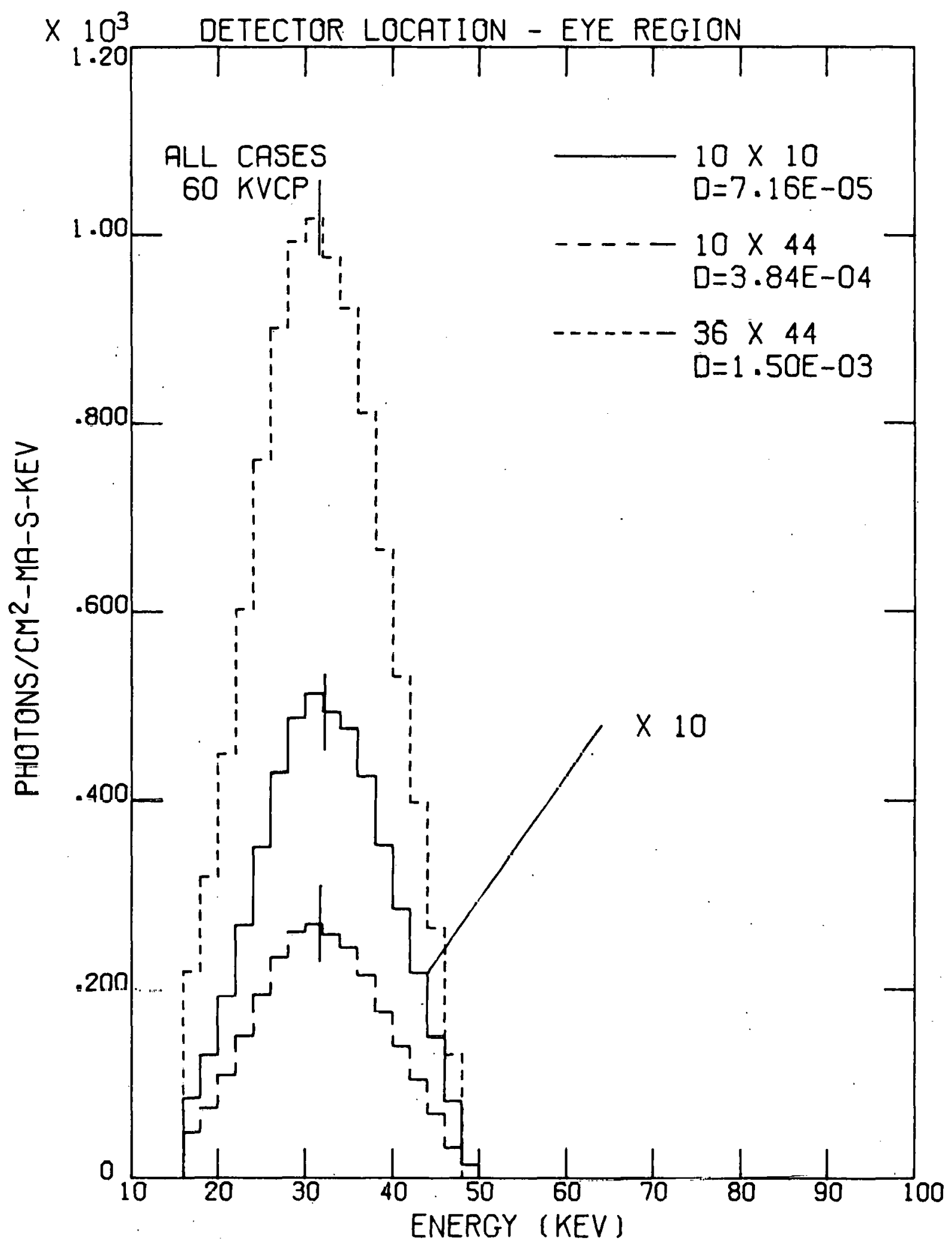

Figure 119. Spectrum at the Eye Region as a Function of Field Size -$60 \mathrm{kV} c \mathrm{p}$. Vertical ticks indicate mean energy. Field size is in $\mathrm{cm}$. $D$ is the absorbed dose per unit current per unit time $(\mathrm{mrad} / \mathrm{mA}-\mathrm{s})$. 


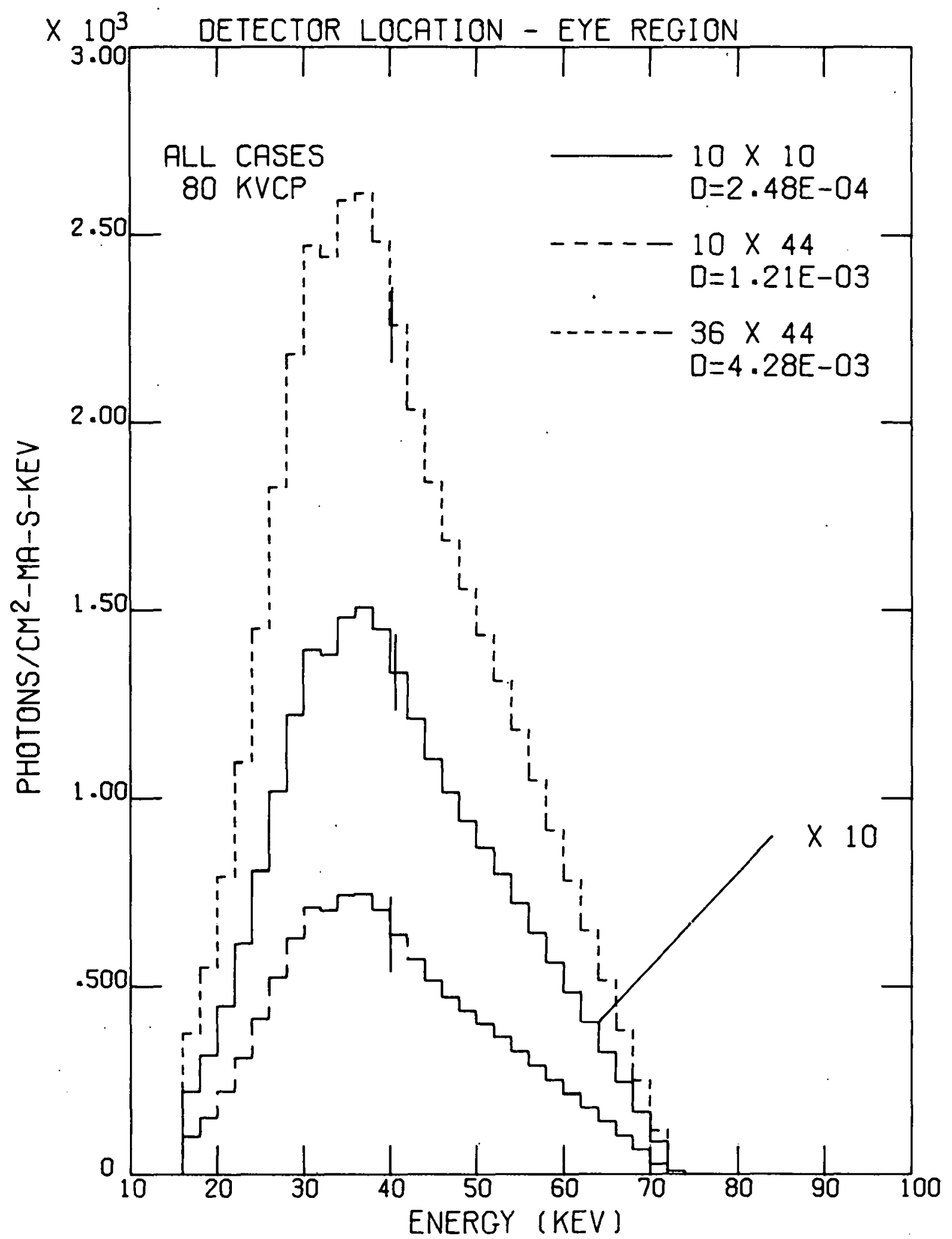

Figure 120. Spectrum at the Eye Region as a Function of Field Size -$80 \mathrm{kV} c p$. Vertical ticks indicate mean energy. Field size is in $\mathrm{cm}$. D is the absorbed dose per unit current per unit time $(\mathrm{mrad} / \mathrm{mA}-\mathrm{s})$. 


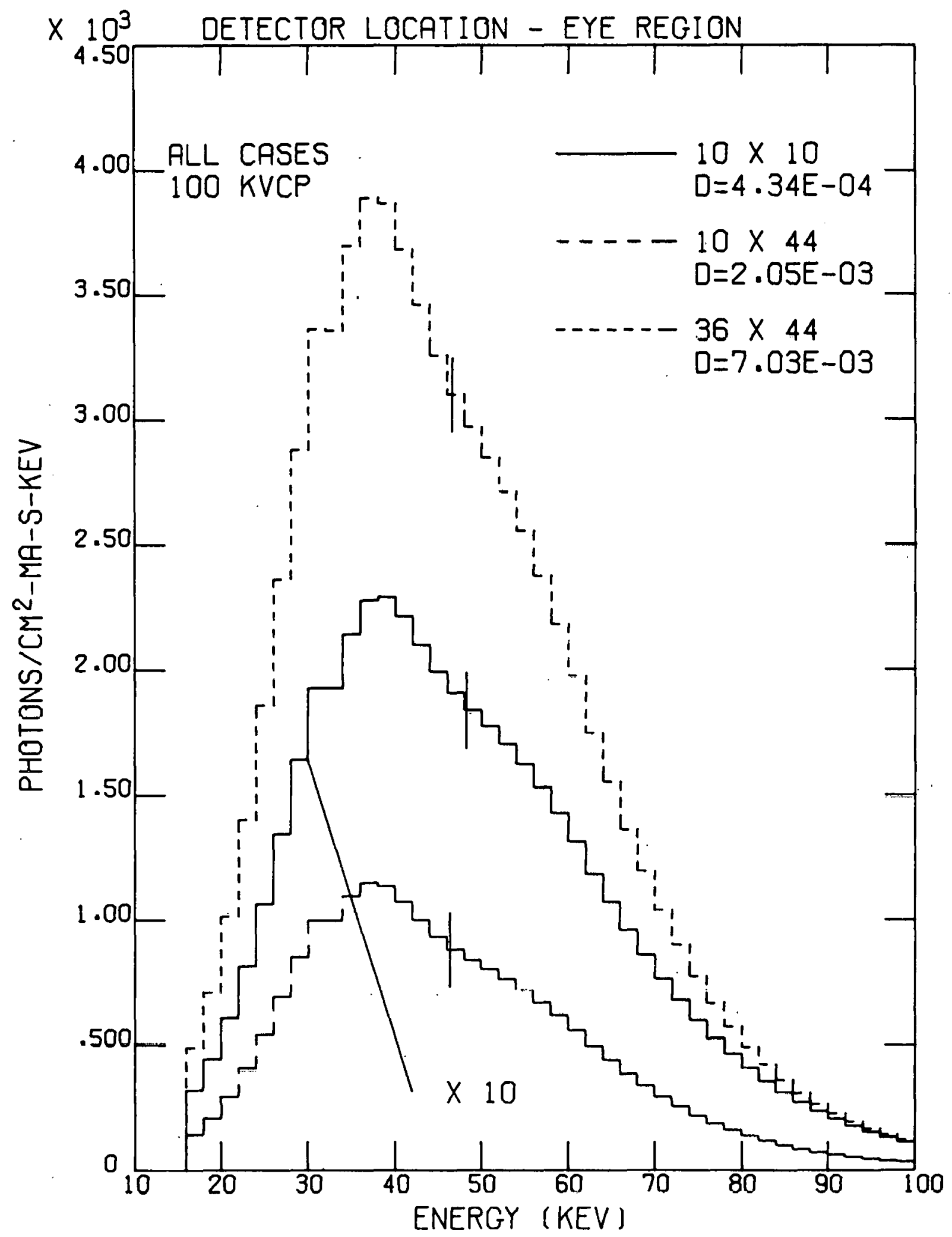

Figure 121. Spectrum at the Eye Region as a Function of Field Size -$100 \mathrm{kVcp}$. Vertical ticks indicate mean energy. Field size is in $\mathrm{cm}$. $D$ is the absorbed dose per unit current per unit time $(\mathrm{mrad} / \mathrm{mA}-\mathrm{s})$. 


\section{BIBL IOGRAPHY}

Al66 Alderson, S. W., 1966, "The role of Phantoms, Machlett-Alderson Phantom," Cathode Press 23, 2.

An76 Anderson, V. E., 1976, personal communication, December 2, 1976.

Ar57 Ardran, G. M., and Crooks, H. E., 1957, "Gonad Radiation from Diagnostic Procedures," Br. J. Radiol. 30, 295.

At75 Atkins, H. L., Fairchild, R. G., Robertson, J. S., and Greenburg, D., 1975, "Effect of Absorption Edge Filters on Diagnostic X-Ray Spectra," Radiol. 115, 431.

BEIR72 BEIR, 1972, National Academy of Sciences-National Research Council Advisory Committee on the Biological Effects of Ionizing Radiations, The Effects on Populations of Exposure to Low Levels of Ionizing Radiation (Washington, NAS-NRC).

Be76 Bemis, C. E., 1976, personal communication, June 10, 1976.

Bi57 Billings, M. S., Norman A., and Greenfield, M. A., 1957, "Gonad Doses During Routine Roentgenography," Radiol. 69, 37.

Ca75 Carter, L. L., and Cashwe11, 1975, Particle Transport Simulation with the Monte Carlo Method, (USERDA Div. Technical Info., TID$2 6 6 0 7 \longdiv { \text { , } 1 6 . }$

Ch70 Chambers, F. W., 1970, Class notes from "Particle Accelerators," Nucl. Eng. 641, Ga. Inst. of Tech. Fal1, 1970.

Du63 Duggan, H. E., and 01de, G. L., 1963, "Radiation Dose to the Skin and to the Gonads from Diagnostic Procedures," Canad. Med. Assoc. J., 89,1144 .

E143 Ellinger, F., 1943, "Lethal Dose Studies with X-Rays I," Medical Times, May, 157.

E161 Ellis, R. E., 1961, "British Survey of the Radiation Dosage to the Population from Medical Radiology, Laboratory Investigations," in the Ninth Int' 1 . Congress of Radiology 1, 112.

Ep67 Epp, E. R., and Weiss, H., 1967, "Spectral Fluence of Scattered Radiation in a Water Medium Irradiated with Diagnostic X-Rays," Rad. Res. 30, 129.

Ep61 Epp, E. R., Weiss, H., and Laughl in, J. S., 1961, "Measurement of Bone Marrow and Gonadal Dose from the Chest X-Ray Examination as a Function of Field Size, Field Alignment, Tube Kilovoltage, and Added Filtration," $\mathrm{Br}$. J. Radiol. 34, 85. 
Ev68 Evans, R. D., 1968, "X-Ray and $\gamma$-Ray Interactions," in Radiation Dosimetry Vol. I, 2nd ed., eds. F. h. Attix and W. C. Roesch (New York: Academic Press), 93.

Ev65 Evans, R. D., 1965, The Atomic Nucleus (New York: McGraw-Hi11), Chap. 21, 23, 24, and 25.

Fi67 Fisher, Jr., H. L., and Snyder, W. S., 1967, "Distribution of Dose in the Body from a Source of Gamma Rays Distributed Uniformly in an Organ," Health Phys. Div. Ann. Progr. Rept., ORNL-4168, 245.

Fr66 Friedlander, G., Kennedy, J.W., and Miller, J. M., 1966, Nuclear and Radtochemtstry, 2nd ed. (New York: Wi ley), Chap. 4.

Ga75 Garry, S. M., Stansbury, P. S., and Poston, J. W., 1975, "Measurement of Absorbed Fractions for Photon Sources Distributed Uniformly in Various Organs of a Heterogeneous Phantom," Health Phys. 28, 591.

Gi75 Gibson, J. A. B., Ardran, G. M., Peaple, L. H. J., Marshall, M., Crooks, H. E., and Birch, R., 1975, "Standardization of the Output from Diagnostic X-Ray Generators," in Biomedical Dosimetry, Symp. Proc. on Advances in Biomedical Dosimetry, held in Vienna, March, 1975, ISBN 92-0-01050575-0, pp. 509-519.

Gr60 Great Britain Committee on Radiological Hazards to Patients, 1960, Radiological Hazards to Patients (London: Her Majesty's Stationery Office).

Gr51 Greening, J.R.,1951, "A Method of Determining the Wavelength Distribution of the $X$-Radiation at a Point in a Scattering Medium," Br. J. Radiol. 24, 204.

Ha72 Hashizume, T., Kato, Y., Maruyama, T., Kumamoto, Y., Shiragai, A., and Nishimura, A., 1972, "Population Mean Marrow Dose and Leukemia Significant Dose from Diagnostic Medical X-Ray Examinations in Japan, 1969," Health Phys. 23, 845.

He74 Heath, M. T., 1974, The Numerical Solution of I1l-Conditioned Systcms of Linear Equations, ORNL-4957.

Hu74 Hurst, G. S., Garrett, w. R., and Payne, M. G., 1974, "Rationale for Radiation Protection," Health Phys. 26, 313.

ICRP66 International Commission on Radiological Protection, 1966, ICRP Publication No. 9, Recommendations of the International Commission on Radiologica Protection, p. 1, part 3, (Oxford: Pergamon Press).

ICRP75 International Commission on Radiological Protection, 1975, Report of the Task Group on Reference Man, ICRP Publication 23, (0xford: Pergamon Press). 
ICRU71 International Commission on Radiation Units and Measurements, 1971, Radiation Quantities and Units, ICRU Report 19.

ICRU28 International Commission on Radiation Units and Measurements, 1928, "International X-Ray Unit of Intensity," Br. J.. Radiol. 1, 363.

Ja67 Jackson, W., 1967, "Dose Assessment in Diagnostic Radiology," Br. J. Radiol. 40, 301.

Ja71 Japan Research Group on the Genetically Significant Dose by Medical Use of X-Ray in Japan, 1961, "The Genetically Significant Dose by the X-Ray Diagnostic Examinations in Japan," Nippon Acta Radiologica 21, 567 .

Jo69 Johns, H. E., and Cunningham, J. R., 1969, The Physics of Radiology, 3rd ed. (Springfield, IL: Thomas).

Jo49 Jones, D. E. A., and Raine, H. C., 1949, "Mix D," Br. J. Radiol. 22, 549 .

Jo76 Jones, T. D., 1976, A CHORD Simulation for Insult Assessment to the Red Bone Marrow, ORNL-5197.

Jo75 Joseph, P. M., 1975, "Mathematical Method for Determining kVp from X-Ray Attenuation Measurements," Med. Phys. 2, 201.

La57 Laught in, J. S., Meurk, M. L. Pullman, J., and Sherman, R. S., 1957, "Bone, Skin, and Gonadal Doses in Routine Diagnostic Procedures," Am. J. Roent. 78, 961.

Le67 Lederer, M. C., Hollander, J. M., and Perlman, I., 1967, Table of Isotopes, 6th ed. (New York: Wiley).

Li58 Lincoln, T. A., and Gupton, E. D., 1958, "Radiation Doses in Diagnostic X-ray Procedures," Radiol. 7l, 208.

Ma60 Mak, S., and Cormack, D. V., 1960, "Spectral Distribution of Scattered X Rays at Points Lying Off the Beam Axis," Br., J. Radiol. 33, 362 .

Mc72 McCorkle, D. L., Christophorou, L. G., and Anderson, V. E., 1972, "Low Energy $(<1 \mathrm{eV})$ Electron Attachment to Molecules at Very High Gas Densities: $0_{2}, " \underline{\mathrm{J}}$. Phys. B: Molec. Phys. $\underline{5}, 1211$.

Mc69 McMaster, W. H., Del Grande, N. K., Mallet, J. H., and Hubbell, J. H., 1969, Compilation of X-Ray Cross Sections, UCRL-50174, Sec. II, Rev. 1.

Me75 Mei, N. H., 1975, Measurement of Absorbed Fractions as a Function of Source Organ Size for Selected Source organs in a Heterogeneous Phantom (Thesis, University of Tennessee). 
Mo71 Morgan, K. Z., 1971, "Excessive Medical Diagnostic Exposure," 3rd Ann. Nat. Conf. on Rad. Control, May 2-6, 1971, Scottsdale, Arizona.

Mo67 Morgan, K. Z., and Turner, J. E., (eds.), 1967, Principles of Radiation Protection (New York: Wiley), Chapter $\bar{T}$.

Mu27 Muller, H. J., 1927, "Artificial Transmutation of the Gene," Science 66,84 .

Mu62 Murray, R. B., 1962, "Scintillation Counters," in Nuclear Instruments and Their Uses, 2nd ed., ed. A. H. Sne11 (New York:WiTey), 82.

Po73 Poston, J. W., and Snyder, W. S., 1973, "A Model for Exposure to a Semi-Infinite Cloud of a Photon Emitter," Health Phys. 26, 287.

Po74a Poston, J. W., and Warner, G. G., 1974, "Absorbed Dose to Selected Internal Organs from Typical Diagnostic Exposures," Proceedings of the Third Congress of the International Radiation Protection Association, Washington, D.C., Sept. 9-14, 1973.

Po74b Poston, J. W., Warner, G. G., and Stansbury, P. S., 1974, Medical $X$-Ray Dose Estimation Program Progress Report for Period Ending August 31, 1974, ORNL/TM-4722.

Pr64 Price, W. J., 1964, Nuclear Radiation Detectors, 2nd ed., (New York: McGraw-Hill), 159.

Qu37 Quimby, E. H., and Arneson, A. N., 1937, "Comparison of Paraffin and Water Phantoms for Roentgen-Ray Depth Dose Measurements," Am. J. Roent. 37, 93.

Ri72 Rindi, A., and Thomas, R. H., 1972, "Povera E Nuda Vai, Dosimetria," Health Phys. 23, 715.

Ro68a Roesch, W. C., and Attix, F. H., 1968, "Basic Concepts of Dosimetry," in Radiation Dosimetry, Vol. I, 2nd ed., eds. F. H. Attix and W. C. Roesch, (New York: Academic Press), 1.

Roh8h Rnesch, W. C., 19К8. "Mathematical Theory of Radiation Fields." in Radiation Dosimetry Vol. I, 2nd ed., eds. F. H. Attix and W. C. Roesch, (New York: Academic Press), 266.

Ko76a Rosenstein, M., 1976, Organ Doses in Diagnost1c Radtology, HEW Publication (FDA) $76-8030$.

Ro76b Rosenstein, M., 1976, Handbook of Selected Organ Doses for Projections Common in Diagnostic Radiology, HEW Publication (FDA) $76-8031$.

Sc97 Scott, N. S., 1897, "X-Ray Injuries," Am. X-Ray J. ‥ 57.

Si77 Siedban. J. M., 1977. University of Wisconsin, personal Communications March 17, 1977. 
Sn74 Snyder, W. S., Ford, M. R., Warner, G. G., and Watson, S. B., 1974, A Tabulation of Dose Equivalent per Microcurie-Day for Source and Target organs of an Adult for various Radionuclides, ORNL/TM-5000.

Sn73. Snyder, W. S., 1973, "Mathematical Procedures for Estimating Dose from an Internally Deposited Emitter," p. 33 in Health Physics Problems of Internal Contamination, (Budapest, Hungary: Akademiai Kiado).

Sn70 Snyder, W. S., 1970, Medical Radionuclides: Radiation Dose and Effects (ERDA Div. Technical Info., Oak Ridge, September 1970), 33.

Sn69 Snyder, W. S., Ford, M. R., Warner, G. G., and Fisher, H. L., Jr., 1969, MIRD Pamphlet №. $\underline{5}$, J. Nucl. med. 10, Supp1. No. 3.

Sp43 Spiers, F. W., 1943, "Materials for Depth Dose Measurement," Br. J. Radiol. 16, 90 .

St55 Stanford, R. W., and Vance, J., 1955, "The Quantity of Radiation Received by the Reproductive Organs of Patients During Routine Diagnostic X-Ray Examinations," Br. J. Radiol. 28, 266.

Sta75 Stansbury, P. S., and Poston, J. W., 1975, "An Outline of Experimental Programs Using the MR. ADAM Phantom," p. 262 in Health Phys. Div. Ann. Progr. Rept., ORNL-5046.

St74 Stansbury, P. S., 1974, "TECALC - A Program to Calculate Compton, Coherent, and Photoelectric Mass Attenuation Coefficients for Photons with Energies Less than $1 \mathrm{MeV}$ and to Assist in the Formulation of Photon-Equivalent Materials," ORNL/TM-4451.

Str75 Strickert, T., and Fosmark, H., 1975, "Some Measurements of Scattered Radiation from an Alderson Phantom for X-Ray Beams from 50 to $200 \mathrm{kVp}, "$ Health Phys. 28, 209.

Ta72 Takeshita, K., Antoku, S., and Sawada, S., 1972, "Exposure Pattern, Surface, Bone Marrow, Integral and Gonadal Doses from Fluoroscopy," Br. J. Radiol. 45, 53.

Th75 Thorngate, J. H., 1975, "Though It's Been Said Many Times, Many Ways ...," Health Phys. 28, 633.

Tr72 Trout, E. D., and Kelley, J. P., 1972, "Scattered Radiation from a Tissue-Equivalent Phantom for X Rays from 50 to $300 \mathrm{kVp}$, "Radiol. $104,161$.

Tr65 Trout, E. d., and Kelley, J. P., 1965, "Scattered Radiation in a Phantom from Diagnostic Quality Radiation," Radiol. 85, 546. 
Va72 Vacirca, S. J., Thompson, D. L., Pasternak, B. S., and Blatz, H., 1972, "A Film-Thermoluminescent Dosimetry Method for Predicting Body Doses Due to Diagnostic Radiography," Phys. Med. Biol. 17, 71:

Wa73 Waggener, R. G., Levy, L. B., and Zanca, P., 1973, "Spectra of Scattered Diagnostic X-Rays from an Alderson Phantom," Health Phys. 24, 59.

Wa72 Waggener, R. G., Roberts, L. F., and Zanca, P., 1972 "A Matrix Method to Correct for Non-Linear Energy Response of $\mathrm{NaI}(\mathrm{Tl})$ Spectrometer Systems," Health Phys. 22, 292.

We57 Webster, E.W., and Merri11, 0. E., 1957, "Measurement of Gonadal Dose in Radiographic Examinations," New England J. of Medicine 257, 311 .

Yo67 Yoshinaga, H., Takeshita, K., Sawada, S., Russe1 1, W. J., and Antoku, S., 1967, "Estimation of Exposure Pattern and Bone Marrow and Gonadal Doses During Fluorscopy," Br. J. Radiol. 40, 344. 
ORNL/TM-5873

Dist. Category UC-41

INTERNAL DISTRIBUTION

1-2. Central Research Library

3. Document Reference Section

4-6. Laboratory Records Department

7. Laboratory Records, ORNL R.C.

8. ORNL Patent Office

9. V. E. Anderson

10. J. A. Auxier

11. S. I. Auerbach

12. C. E. Bemis

13. W. F. Bunch

14. H. M. Butler

15. D. J. Christian

16. K. E. Cowser

17. F. L. Culler

18. D. M. Davis

19. H. W. Dickson

20. M. R. Ford

21. W. R. Garrett

22. R. E. Goans

23. W. M. Good
24. F. F. Haywood

25. R. F. Hibbs

26. T. D. Jones

27. S. V. Kaye

28. G. D. Kerr

29. S. R. Larkins

30. F. D. Mundt

31. W. F. Ohnnesorje

32. W. W. Parkinson

33. S. K. Penny

34. P. T. Perdue

35-39. J. K. Poggenburg

40. H. Postma

41. C. R. Richmond

42. B. S. Shoup

43. J. B. Storer

44. J. H. Thorngate

45. G. G. Warner

46. H. A. Wright

\section{EXTERNAL DISTRIBUTION}

47. M. W. Carter, Georgia Institute of Technology, School of Nuclear Engineering, Atlanta, Ga. 30332.

48. J. R. Cameron, University of Wisconsin, Department of Radiology, University Hospital, 1300 University Avenue, Madison, Wis. 53706.

49. F. W. Chambers, Georgia Institute of Technology, School of Nuclear Engineering, Atlanta, Ga. 30332.

50. W. L. Chen, Lung-Tan, P.0. Box 3, Taipei, Taiwan, Republic of China 325.

51. R. E. Christman, University of North Carolina at Chapel Hill, Department of Environmental Science and Engineering, School of Public Health, Chapel Hill, N.C. 27514.

52. R. J. Cloutier, ORAU, Oak Ridge, Tenn. 37830.

53. M. V. Davis, Georgia Institute of Technology, School of Nuclear Engineering, Atlanta, Ga. 30332.

54. S. F. Deus, Coordenadoría de Protecáo Radiológica E Dosimetria, Instituto de Energia Atómica, Cidade Universitária, C.P. 11049, Pinheiros, São Paulo, S.P. Brazil.

55. Director, Research and Technical Support Division, ERDA-ORO.

56. G. G. Eichholz, Georgia Institute of Technology, School of Nuclear Engineering, Atlanta, Ga. 30332.

57. R. H. Fetner, Georgia Institute of Technology, School of Nuclear Engineering, Atlanta, Ga. 30332. 
58. H. L. Fisher, Jr., Experimental Biology Laboratory EPA, Research Triangle Park, N.C. 27711.

59. S. M. Garry, TVA-River Oaks Building, Muschle Shoals, Ala. 35660.

60. M. J. Haire, Georgia Institute of Technology, School of Nuclear Engineering, Atlanta, Ga. 30332.

61. D. P. Humer, Georgia Institute of Technology, School of Nuclear Engineering, Atlanta, Ga. 30332

62. J.M. Kallfelz, Georgia Institute of Technology, School of Nuclear Engineering, Atlanta, Ga. 30332.

63. Dr. James Kereiakes, E555 Medical Sciences Building, University of Cincinnati, Cincinnati, Ohio 45267.

64. P. H. McGinley, Georgia Institute of Technology, School of Nuclear Engineering, Atlanta, Ga. 30332.

65. H. N. Mei, P.0. Box 3, Lung-Tan, Taiwan 325, Republic of China.

66. D. W. Moeller, 27 Wildwood Dr., Bedford, Mass. 01730.

67. K. Z. Morgan, Georgia Institute of Technology, School of Nuclear Engineering, Atlanta, Ga. 30332.

68.69. ORNU University Programs Office, Oak Ridge, Tenn. 37830.

70. Dr. Peter Paras, Bureau of Radiological Health, Food and Drug Administration, 12720 Twinbrook Parkway, Rockville, Md. 20852.

71. J.W. Poston, Georgia Institute of Technology, School of Nuclear Engineering, Atlanta, Ga. 30332.

72-81. M. Rosenstein, 6 Gruenther Court, Rockville, Md. 20851.

82. B. Saylor, University of North Carolina at Chapel Hi11, Department of Radiology, School of Medicine, Chapel Hill, N.C. 27514.

83. A. Schneider, Georgia Institute of Technology, School of Nuclear Engineering, Atlanta, Ga. 30332.

84. R. H. Schnieder, Bureau of Radiological Health, Food and Drug Administration, 12720 Twinbrook Parkway, Rockville, Md. 20852.

85. B. Shleien, 73 Fuller St., Brookline, Mass. 02146.

86. J. M. Siedband, University Avenue, Madison, Wis. 53706.

87-94. P. S. Stansbury, Department of Environmental Sciences and Engineering, School of Public Health, University of North Carolina at Chapel Hi11, Chapel Hill, N.C. 27514.

95. J. E. Watson, Jr., University of North Carolina at Chapel Hill, Department of Environmental Science and Engineering, School of Public Health, Chapel Hill, N.C. 27514.

96. L. E. Weaver, Georgia Institute of Technology, School of Nuclear Enqineering, Atlanta, Ga. 30332.

97. W. W. Weyzen, Radiological Physics and Instrumentation Branch, Division of Biomedical and Environmental Research, U.S. Environmental Research Development Administration, Washington, D.C. 20545.

98. D. R. White, Radiation Physics Department, St. Bartholomew's Hospital, West Smithfield, London EClA7BE, England.

99. R. W. Wood, Radiological Physics and Instrumentation Branch, Division of Biomedical and Environmental Research, U.S. Environmental Research Development Administration, Washington, D.C. 20545.

100. John C. Villforth, Bureau of Radiological Health, Food and Drug Administration, 12720 Twinbrook Parkway, Rockville, Md. 21205.

101-368. Given distribution as shown in TID-4500 under Health and Safety category (25 copies - NTIS). 